SLAC-R-631

LBL-91-1996

\title{
Current Experiments in Particle Physics
}

\author{
Particle Data Group LBL-91 Collaboration
}


LBL-91 Revised

UC-414

September 1996

\title{
CURRENT EXPERIMENTS IN PARTICLE PHYSICS
}

\section{Particle Data Group}

\section{H. Galić}

Stanford Linear Accelerator Center, Stanford University, Stanford, CA 94305 USA

\section{F. Lehar}

Centre d'Etudes Nucleaires de Saclay, F-91191 Gif-sur-Yvette, France

\section{V.I. Klyukhin, Yu.G. Ryabov}

Institute for High Energy Physics, RU-142284 Protvino, Moscow Region, Russia

\section{S.V. Bilak, N.S. Illarionova}

Institute of Theoretical and Experimental Physics, RU-117259 Moscow, Russia

\author{
B.A. Khachaturov, E.A. Strokovsky \\ Joint Institute for Nuclear Research, RU-141980 Dubna, Moscow Region, Russia
}

\author{
C. M. Hoffman \\ Los Alamos National Laboratory, Los Alamos, NM 87545, USA \\ P.-R. Kettle \\ Paul Scherrer Institute, CH-5232 Villigen PSI, Switzerland
}

\section{A. Olin}

TRIUMF, 4004 Wesbrook Mall, Vancouver BC V6T 2A3, Canada

\author{
F.E. Armstrong (Technical Associate) \\ Particle Data Group, Lawrence Berkeley National Laboratory, Berkeley, CA 94720, USA
}

\begin{abstract}
This report contains summaries of current and recent experiments in Particle Physics. Included are experiments at BEPC (Beijing), BNL, CEBAF, CERN, CESR, DESY, FNAL, Frascati, ITEP (Moscow), JINR (Dubna), KEK, LAMPF, Novosibirsk, PNPI (St. Petersburg), PSI, Saclay, Serpukhov, SLAC, and TRIUMF, and also several proton decay and solar neutrino experiments. Excluded are experiments that finished taking data before 1991. Instructions are given for the World Wide Web (WWW) searching of the computer database (maintained under the SLAC-SPIRES system) that contains the summaries.
\end{abstract}

The publication of this report is supported by the Director, Office of Energy Research, Office of High Energy and Nuclear Physics, the Division of High Energy Physics of the U.S. Department of Energy under Contract No. DEAC03-76SF00098, and by the U.S. National Science Foundation under Agreement No. PHY-9320551. Partial funding to cover the cost of the publication is also provided by an implementing arrangement between the governments of Japan (Monbusho) and the United States (DOE) on cooperative research and development. H. Galic is supported by the U.S. Department of Energy under Contract No. DE-AC03-76SF00515. 


\section{TABLE OF CONTENTS}

Introduction $\ldots \ldots \ldots \ldots \ldots \ldots \ldots \ldots$

Searching the EXPERIMENTS computer database $\ldots \ldots \ldots .3$

Spokesperson index $\ldots \ldots \ldots \ldots \ldots \ldots \ldots$

Abbreviations used in the summaries

Journals . . . . . . . . . . . . . . . . . . . . . . 12

Kinematic variables $\ldots \ldots \ldots \ldots \ldots \ldots \ldots \ldots$

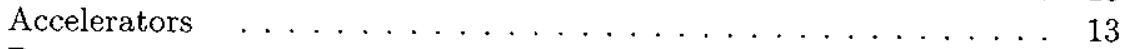

Detectors ....................... 14

Summaries of experiments

BEPC (Beijing) $\ldots \ldots \ldots \ldots \ldots \ldots \ldots \ldots \ldots \ldots \ldots \ldots \ldots$

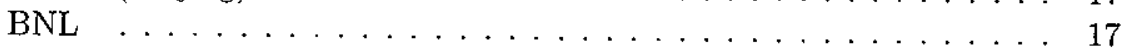

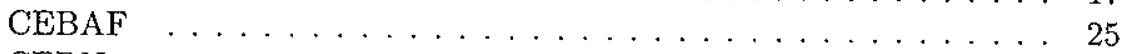

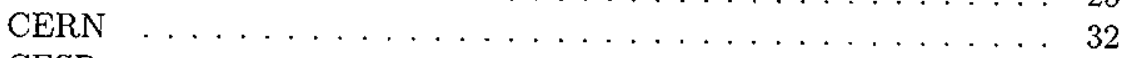

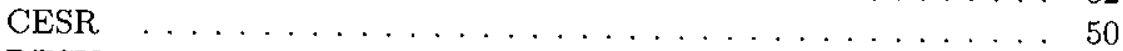

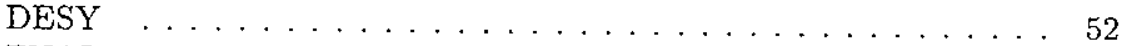

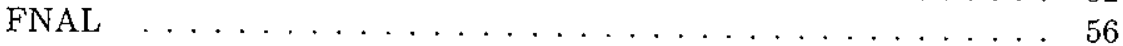

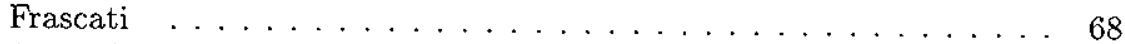

$\operatorname{ITEP}($ Moscow) $\ldots \ldots \ldots \ldots \ldots \ldots \ldots . \ldots \ldots \ldots$

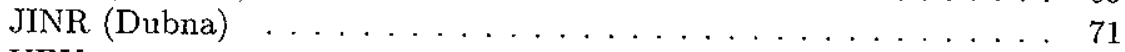

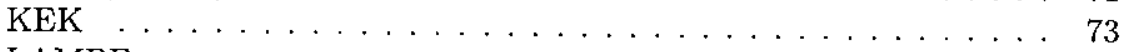

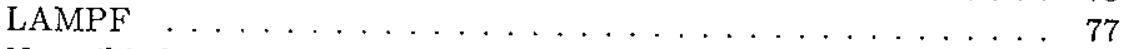

Novosibirsk . . . . . . . . . . . . . . . 80

PNPI (St. Petersburg) $\ldots \ldots \ldots \ldots \ldots \ldots \ldots \ldots$

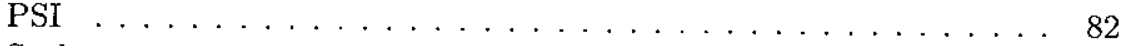

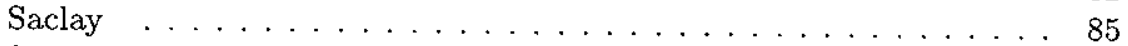

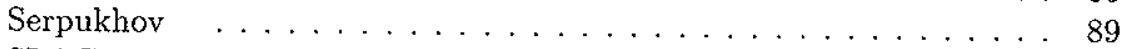

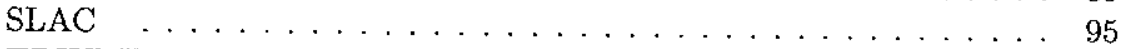

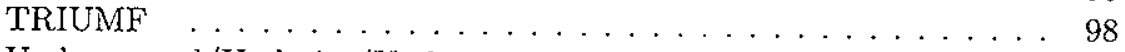

Underground/Underice/Underwater $\ldots \ldots \ldots \ldots \ldots 10 \ldots \ldots$ 


\section{INTRODUCTION}

This report contains full summaries of 180 approved current and recent experiments in elementary particle physics. The focus of the report is on selected experiments which directly contribute to our better understanding of elementary particles and their properties such as masses, widths or lifetimes, and branching fractions. This year's report is, in a way, a companion volume to the main Particle Data Group publication, Review of Particle Physics. Experiments at the following laboratories are included:

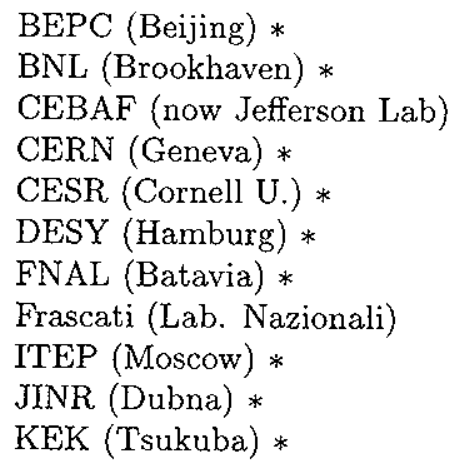

\author{
LAMPF (Los Alamos) * \\ Novosibirsk (Inst. Nucl. Phys.) \\ PNPI (St. Petersburg) \\ PSI (Villigen) * \\ SATURNE (Saclay) * \\ Serpukhov (Inst. High Energy Phys.) \\ SLAC (Stanford U.) * \\ TRIUMF (Vancouver) \\ Underground experiments \\ Underice experiments \\ Underwater experiments
}

For the institutions marked with an asterisk, the corresponding summaries section also contains a brief description of the particle physics plans at the laboratory till the end of the century.

We also list 263 other experiments at the above laboratories. Although, to the best of our knowledge, these experiments do not measure directly the properties of particles listed in the Review of Particle Physics, they still may be of considerable interest to the particle physics community. These additional experiments only have brief entries in the printed version of the report, but often have a full description in an online-accessible database from which the report is produced. The readers are encouraged to find more information about those experiments in the database (see below).

We exclude from this year's report the experiments for which the data collection was completed before 1991. We generally do not cover the experiments dealing primarily with nuclear levels or other nuclear-structure properties. As to the choice of institutions, we try to include those in which particle physics experiments top the list of priorities at the laboratory.

Sources of information - Our first information about an experiment usually comes from the proposal for the experiment. Subsequently, we follow the progress of the experiment as best we can in laboratory reports such as Experiments at CERN. Finally, a few months before an edition of this report is to appear, we send copies of the summaries of the experiments to the spokespersons for checking and updating. If a reply is received — as was the case for $90 \%$ of the experiments there is a " $\checkmark$ " next to the spokesperson's name. Since current experiments are often in flux, we rely heavily on these replies to be up to date: no $\checkmark$ by the spokesperson means the summary may be inaccurate or incomplete. (For a handful of experiments, we verified our information with a senior member of the experiment, not the spokesperson, but for simplicity put a $\checkmark$ by the spokesperson. For experiments with more than one spokesperson, all the spokespersons are checked even if only one of them replied.)

Computer database - This report is produced from a computer database maintained at SLAC under the SPIRES database management system. The database, named EXPERIMENTS, also contains information from earlier editions of this report, going back to about 1975, and including older experiments at Argonne, INS, IUCF, and Rutherford. See page 3 for a guide to using the EXPERIMENTS database via the World Wide Web (WWW). 
Summaries - Each summary lists several dates related to the experiment: the date of the proposal, the approval date, and when the data-taking began and was completed. The title of the proposal and the most recent list of participants are given. The detector used in the experiment is identified either by a generic name (e.g., counter) or by a widely known acronym (e.g., SLD). The most important reactions and particles studied and the beam energy or momentum are listed where known. A brief comment describing the apparatus and the main goals of the experiment may follow. A summary ends with a list of any journal articles on results or instrumentation of the experiment. Related experiments, similar either in methods used or in subject of study, are also listed. Where known, an e-mail contact address and the WWW uniform resource locator (URL) are given.

Abbreviations - To keep the summaries brief, abbreviations are used to indicate journals, kinematic variables, accelerators, and detectors. The abbreviations are usually obvious but are also defined near the beginning of the report. The abbreviated forms are needed for searching the EXPERIMENTS database online.

Acknowledgments - P. Yamin (BNL), D. Buckle (CEBAF), M. Draper and B. Powell (CERN), and J. Parker (FNAL) kindly provided computer files with data on experiments from their respective institutions. Valuable additional information was obtained from P. Yamin (BNL), G. Fraser (CERN), P. Drell (CESR), P. Folkerts (DESY), R. Rubinstein (FNAL), M.V. Danilov (TTEP), H.K. Walter (PSI), and D.W. Leith (SLAC). We thank F.E. Armstrong (LBL) for her help with the final processing of the manuscript. We particularly thank the hundreds of spokespersons who took the time to reply to our inquiries.

Comments and requests - We invite comments pointing out omissions, obscurities, outof-date information, and errors. We also encourage spokespersons to send us proposals and letters of intent for their future experiments. Comments and other material should be sent to:

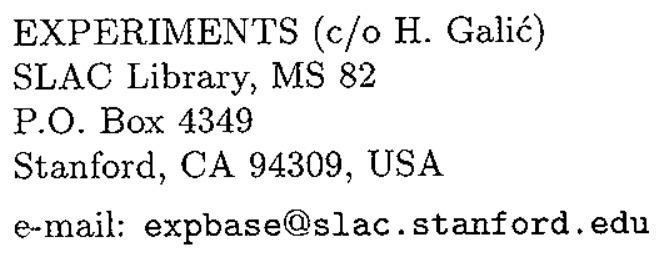

To order additional copies of the Current Experiments from North and South America, Australia, and the Far East write to:

CURRENT EXPERIMENTS

Particle Data Group, MS 50-308

Lawrence Berkeley National Laboratory

Berkeley, CA 94720, USA

e-mail: pdg@lbl.gov

Requests from all other areas should go to:

CERN Scientific Information Service

CH-1211 Geneva 23

Switzerland

http://wwwas . cern. ch/library/

(select - Ordering CERN Publications) 


\section{SEARCHING THE 'EXPERIMENTS' DATABASE VIA WORLD WIDE WEB}

The summaries of current and many earlier experiments related to particle physics are contained in a computer database called EXPERIMENTS, maintained at SLAC under the SPIRES database management system. Note that even the experiments which only have a brief description in this report may still have a full description in the database. You can access the EXPERIMENTS database most easily via the World Wide Web (WWW). The first part of this section describes a simple database search based on the Web forms. This is followed by a few examples of a more advanced search technique. The concluding part lists some other computer-reachable sources of interest to experimental particle physicists.

General information - If your computer is linked to the Internet, you should be able to access the World Wide Web. The Web was brought to life in the early nineties by Timothy Berners-Lee and collaborators at CERN. Free WWW software is available for various platforms and various needs, from PC's to large computer systems, from simple line-mode browsers to sophisticated full-screen navigators. Names of some of the popular software packages that enable the Web access are Lynx, Netscape, and Mosaic, but the selection is by no means restricted to those three. Learn from your local computing center where and how to obtain the Web software most appropriate for your computer.

EXPERIMENTS home page --- The EXPERIMENTS database and many other SPIRES-based SLAC Library databases are searchable via WWW. Find the home page for the EXPERIMENTS database at:

\section{http://www-spires.slac.stanford.edu/find/experiments}

When you open this document, you find a simple form which allows you to fill in one or several elements which best describe your search criteria. In the old days, when the database was only accessible through e-mail, you had to send a request written in the SPIRES search language to the SPIRES e-mail server. The Web form eliminates this step, and even a novice can immediately begin a searching session. Furthermore, a Web search presents much more than the old e-mail search method. The e-mail result was a text, while the WWW result is a hypertext. The marked hypertext leads to related links, and you can, for example, find a more thorough bibliographic description of journal articles corresponding to a given experiment (from the HEP database), and often read or print a postscript version of such articles. Similarly, if an experiment has a Web home page, you will be able to get there easily, and you may also find a direct link to the database entries of the experiments related to the selected one.

Simple form searching - Forms, in general, are simple to use and don't require much explanation. Explore them on your own, or use the following hints to get to the expected result even sooner. The EXPERIMENTS form allows you to find a result by a title-word search, by a search for a participant in an experiment, or by the experiment's official code number. You can also find all the experiments in which a certain institution is involved, or your search could be based on, for example, the particles studied in an experiment. You fill in the entries you know, leaving the others blank. The 'result' of a search is a database entry which fully describes an experiment, including the complete list of authors, a title, a description of the experiment's goals and methods, a list of resulting journal articles, etc.

To find all the experiments in which the author Johnson has participated, type the author's last name (surname) into the appropriate box. If an initial is added, separate it by a comma, e.g., johnson, g. Keep in mind that somebody you may know as Betty could officially be Elizabeth, Bob may be signing his papers as Robert, etc. If you are not sure what the right first name is, just do not use the initial. The last name can be truncated, e.g., leder\# for Lederman. To deal with a combination of several authors, use the words and, or, and and not, e.g., johnson or smith will yield experiments by either physicist, johnson and smith will find experiments in which both have participated, whereas johnson and not smith will yield experiments by Johnson so long as Smith is not involved. If a special character appears in a name, such as the $\ddot{u}$ in the German spelling of Müller, try alternate spellings, such as muller or mueller. If you happen to know that author Johnson is from SLAC, for example, use both the participant and the affiliation boxes. If you are only interested in experiments which author Johnson did at Fermilab, type in the name (johnson), and also select Fermilab in the list of accelerators. To initiate a search, press the Perform Search button. Do not forget to use the Clear All Fields button between two searches. 
If you are interested in the experiments that study the CP violation, for example, try the title-word search. Enter any or all expected words from the title in any order. A truncated search may be used, e.g., $\mathrm{cp \#}$ in the title-word box yields titles containing the word CP standing free (as in CP violation), and also all the titles in which CP is hyphenated (as in CP-violating). If you type solar neutrino into the title-word box, you will find all the experiments with these two words (any order) in the titles. You can combine any number of other elements with your title-word search. For example, to find some of the recently approved CP violation experiments, type cp\# in the title-word box, and select 'After' and '1990' in the two pull-down menus for the date-approved search element.

To find all the experiments in which people from a given institution participated, type the institution's name (exactly as it appears in this report's summaries) into the affiliation box. Similarly, type the experiments' official code number, as it appears in this report, into the exp-number box (e.g., type cern-na-048 to find more about the NA48 experiment at CERN). For many experiments you can also use a nickname instead of the complete code number. For example, if you type opal in the exp-number box, you will find the CERN-LEP-OPAL entry, while $\underline{\text { do }}$ or dzero yields the FNAL-740 description. (You can also find the OPAL and D0 entries by selecting the corresponding detectors in the list of detectors). To find both D0 and CDF entries, use dzero or cdf, and to find all four LEP experiments, type cern-lep-\# into the exp-number box.

To use the studied-particle box, you must know the database-adopted spelling of various particles. We use $\underline{\mathrm{k}+}$, for example, and not kplus for $K^{+}$, and pio, not pizero for $\pi^{0}$. An antiparticle name in the database is usually formed by adding the suffix bar to the corresponding particle name. Thus the antiproton in the studied-particle box should be written as pbar. An extensive list of the 'correct' particle names can be found on the EXPERIMENTS help page. (Note that the particle names in the studied-particle search are strictly standardized. In a title search, to the contrary, particle names are somewhat variable in their spelling and several forms should be tried).

If you are interested in finding all the experiments at a given accelerator, select the appropriate laboratory in the list of accelerators. Similarly, if you know a detector's name, try the list of detectors. The defaults are 'Any Accelerator' and 'Any Detector'. The date-approved search is useful if you know the approval year. You can also eliminate older experiments from your search result if you select the date-approved carefully. The default for the date-approved is 'Don't Know'.

Advanced searching -- For the old hands and for those who want to have better control over searching, the form has a separate part named 'Make your own search'. This part only has one search window into which you type a complete SPIRES search command. To learn more about the SPIRES search language, check the 1994 and earlier editions of Current Experiments. Here we give a few examples of what could be typed into the SPIRES search window. We use lower case, but a search is case insensitive, and in an actual search you may use both lower and upper case characters.

Important note: whenever a term you wish to find contains characters, ')' , '>' , '<' , or '(' , the entire search value must be enclosed in double quotes (see examples below)

FIND, SHOW and BROWSE are some of the command verbs you can use in SPIRES searching. You can formulate a variety of search commands by using these three verbs. For example,

show indexes

(Shows the available search terms, e.g., AUTHOR, PAPER, EXP, etc. Use these terms in your search request to BROWSE and FIND, e.g., BROWSE AUTHOR ..., or FIND PAPER ..., FIND EXP ..., etc.)

browse author trilling.

(Displays values in the author-name index surrounding the stated value.)

browse exp

(No search value for EXP is given. SPIRES returns several random values of experiment codenumbers.) 
browse exp desy

(Displays values in the code-number index alphabetically surrounding the specified value. Useful if you do not know the exact form or spelling of a search value.)

find author rubbia, $c$ or

find author $c$ rubbia

(Finds experiments in which the stated author has participated. The first-name initial is optional:

find author rubbia will also work fine.)

find exp saturne-258

(Finds the record corresponding to the specified value.)

find paper "PR D37 (1988) 1131"

(Finds the experiment reported in the stated reference. Note that the reference has spaces but no commas between the elements. Double quotes are obligatory, see the box above.)

find reaction "e+ $e^{--->m u+~ m u-~} \mathrm{x}$ "

(The 'arrow' is composed of two minus signs and a 'greater-than' sign. Note the obligatory quotes.)

find ac kek-tristan

(Finds experiments using the stated accelerator.)

find de crystal-ball

(Finds experiments using the stated detector.)

find title $c p$ phase difference

(Finds experiments with the words CP, PHASE, and DIFFERENCE in the title. The order of title words in a search command is unimportant.)

find af rutgers $u$

(Finds experiments in which people from the stated institution participated.)

find af rutger\#

(Finds experiments with the stated affiliation. '\#' represents any remaining characters.)

find particle $d+$

(Finds experiments studying the specified particle.)

The following search commands are also allowed:

find author prefix patters

(Finds authors whose last names begin with the string PATTERS, e.g., Patterson)

find af prefix northwest

find reaction prefix pit $n$

find exp sld

(For most of the large experimental collaborations you may type just the collaboration name instead of the full experimental number)

Compound searching is not only possible, but also desirable, because it keeps search results smaller:

find author prokoshkin and exp prefix cern

find af moscow, itep and not date before 1992

find particle $j / p s i$ or "psi(2s)"

Occasionally a search may fail. With some care you can reduce unsuccessful searching to a minimum: (i) Remember that any search value containing any of the special characters $),>,<,($, must be enclosed in double quotes. See the examples above for the PAPER, REACTION, and PARTICLE searches; (ii) Use the 'correct' form of a search value: a database-adopted particle name (e.g., $\mathrm{K}+$, not Kplus), proper experiment code-name (e.g., FNAL-761, not FNAL-E761), etc. To find the correct forms, use the BROWSE command for the index you are searching. 
Other SLAC-SPIRES databases - Several other SLAC Library databases of interest to experimental physicists are available via WWW. They are all listed on the SPIRES home page:

$$
\text { http://www-spires.slac.stanford.edu/find/spires.html }
$$

(1) HEP-PREPRINTS database is a joint project of SLAC and DESY libraries, and contains more than 320,000 bibliographic entries on particle physics papers (preprints, journal articles, reports, theses, conference papers, etc.); (2) BOOKS contains bibliographic summaries of more than 20,000 textbooks, conference proceedings, monographs, etc., covering high-energy physics and related topics; (3) CONFERENCES database lists past and future conferences, schools, and meetings of interest to the particle-physics community; (4) HEPNAMES contains more than 30,000 e-mail addresses of people working in high-energy and medium-energy physics. (By the way, you can retrieve the HEPNAMES data without leaving the EXPERIMENTS search form: to get the author Johnson's e-mail address, for example, just type the command query johnson into the 'Make your own search' window); (5) Database INSTITUTIONS lists about 5,000 addresses (and, often, phone and fax numbers) of institutions related to high-energy physics.

Other computer accessible sources - Clones of the EXPERIMENTS database are maintained for local users at the Yukawa Institute (Kyoto-SPIRES), Durham University (Durham-RAL BDMS), and IHEP (Protvino BDMS). The Durham version can also be found at http://cpt1. dur.ac.uk/HEPDATA/EXP

A Web document called Experiments Online is a guide to home pages of various high-energy physics collaborations. It is updated weekly. To add your experiment's home page to the document, write to expbase@slac.stanford.edu. Find Experiments Online at:

$$
\text { http://www-spires.slac.stanford.edu/find/explist.html }
$$

We urge all active experimental collaborations to establish their home pages on the Web. Such pages keep the collaboration members better informed, and should also serve to represent the group to the world. At the very minimum, each home page should list the current members of the collaboration and identify the spokesperson, give the objectives of the experiment and describe the experimental apparatus, and list the journal articles, conference papers and preprints which have resulted from the collaboration's work.

Experimental physicists are invited to post their papers to the hep-ex e-print archive. To receive detailed instructions on submitting and retrieving papers, send a blank e-mail message with the subject help to: hep-ex@xxx. lanl.gov. E-mail listings of the experimental physics titles and abstracts submitted to the archive can be received daily by sending a blank e-mail with the subject subscribe <your-name> to hep-ex. The listings and papers can also be accessed through the Web link http://xxx. Ianl.gov/

You will find a well-documented list of other computer accessible sources of interest to particle physicists in the latest edition of the Review of Particle Physics, Physical Review D54 (1996) 1-720. The list is also posted at:

$$
\text { http://www.slac.stanford.edu/library/pdg/hepinfo.html }
$$

The Review itself is available over the Web. Check the Berkeley Particle Data Group WWW server at http://pdg.lbl.gov/ 


\section{SPOKESPERSON INDEX}

ABE, K. (KEK, 'Tsukuba) KEK-TE-003 ABEGG, R. (TRIUMF) TRIUMF-482 AMMOSOV, V.V. (Serpukhov, IFVE) SERPUKHOV-145 ANGHINOLFI, M. (INFN, Genoa) CEBAF-93-031 APPEL, J.A. (Fermilab) FNAL-791

ARMSTRONG, D.S. (LBL, Berkeley) TRIUMF-570 AR.NOLD, R.G. (American U.) SLAC-E-143 ARNOLD, R.G. (American U.) SLAC-E-155 ASANO, Y. (Tsukuba U., Inst. Appl. Phys.) KEK-TE-001 ASHERY, D. (Tel Aviv U.) TRIUMF-445 AVIGNONE, F.T. (South Carolina U.) ITEP-896 AVIGNONE, F.T. (South Carolina U.) ITEP-912 AYRES, D.S. (Argonne) FNAL-875

AZHGIREY, L.S. (Dubna, JINR) JINR-LHE-0941-3 AZUELOS, G. (Montreal U.) TRIUMF-452 BADERTSCHER, A. (Zurich, ETH) PSI-R-86-05 BAGHAEI, H. (Virginia U.) CEBAF-89-015 BALDIN, A.A. (Dubna, JINR) JINR-LHE-1010 BALL, J. (Saclay) SATURNE-225

BALTAY, C. (Yale U.) SLAC-SLC-SLD

BARABASH, A.S. (Moscow, ITEP) ITEP-876 BARABASH, A.S. (Moscow, ITEP) ITEP-896 BARISH, B.C. (Cal Tech) UNDERGROUND-MACRO BARNES, P.D. (Los Alamos) BNL-813 BARNES, P.D. (Los Alamos) BNL-836 BARWICK, S. (UC, Irvine) UNDERICE-AMANDA BECK, D.H. (Illinois U., Urbana) CEBAF-91-017 BEIER, E.W. (Penn U.) UNDERGROUND-SUDBURY BEISE, B. (Maryland U.) CEBAF-91-004 BELLETTINI, G. (Pisa U. and SNS, Pisa) FNAL-741 BELOSTOTSKY, S.L. (St. Petersburg, INP) SATURNE-145 BELYAKOV-BODIN, V.I. (Moscow, ITEP) SERPUKHOV-171 BERNSTEIN, R.H. (Fermilab) FNAL-815 BERTIN, P. (Clermont-Ferrand U.) CEBAF-94-002 BERTINI, R. (Saclay) SATURNE-213 BHANG, H.C. (Seoul National U.) KEK-307 BING, O. (Strasbourg, CRN) SATURNE-174 BIRCHALL, J. (Manitoba U.) TRIUMF-497-287 BJORKEN, J.D. (SLAC) FNAL-864 BLANPIED, G. (South Carolina U.) SATURNE-209 BODEK, A. (Rochester U.) SLAC-E-140X BOGGILD, H. (Bohr Inst.) CERN-NA-044 BOGUSLAVSKY, I.V. (Dubna, JINR) SERPUKHOV-161 BOLOTOV, V.N. (Moscow, INR) SERPUKHOV-166 BONNER, B.E. (Rice U.) BNL-817 BONNER, B.E. (Rice U.) BNL-854 BOUDARD, A. (Saclay) SATURNE-145 BOWLES, T.J. (Los Alamos) UNDERGROUND-SAGE BOWMAN, J.D. (Los Alamos) LAMPF-1188 BOYARD, J.L. (Orsay) SATURNE-190 BOYARD, J.L. (Orsay) SATURNE-251 BOYARD, J.L. (Orsay) SATURNE-278 BRACK, J.T. (TRIUMF) TRIUMF-645 BRADAMANTE, F. (Trieste U.) CERN-PS-206 BRAUN-MUNZINGER, P. (SUNY, Stony Brook) BNL-814 BRAUN-MUNZINGER, P. (SUNY, Stony Brook) BNL-877 BREIDENBACH, M. (SLAC) SLAC-SLC-SLD BRESSANI, T. (Turin U.) FRASCATI-DAPHNE-FINUDA BRISCOE, W.J. (George Washington U.) BNL-909 BRISCOE, W.J. (George Washington U.) CEBAF-94-103 BRODZINSKI, R.L. (Battelle Mem. Inst.) ITEP-912 BROOKS, W.K. (CEBAF) CEBAF-94-017 BRYMAN, D.A. (TRIUMF) BNL-787 BUNJATOV, S.A. (Dubna, JINR) SERPUKHOV-136 BURKE, D.L. (SLAC) SLAC-E-144 BURKERT, V.D. (CEBAF) CEBAF-89-037 BURKERT, V.D. (CEBAF) CEBAF-89-038 BURKERX, V.D. (CEBAF) CEBAF-89-042 BURKERT, V.D. (CEBAF) CEBAF-91-002 BURKERT, V.D. (CEBAF) CEBAF-91-023
BURKERT, V.D. (CEBAF) CEBAF-91-024 BURKERT, V.D. (CEBAF) CEBAF-93-006 BURLESON, G.R. (New Mexico State U.) LAMPF-1178 BURLESON, G.R. (New Mexico State U.) LAMPF-1256 BURLESON, G.R. (New Mexico State U.) LAMPF-1267 BUTLER, J.N. (Fermilab) FNAL-687

CALDWELL, A. (Nevis Labs, Columbia U.) DESY-HERA-ZEUS CALVETTI, M. (Florence U. and INFN, Florence) CERN-NA-048 CARITHERS, W. (LBL, Berkeley) FNAL-741 CARROLL, A.S. (Brookhaven) BNL-850 CASON, N.M. (Notre Dame U.) BNL-852 CATES, G.D. (Princeton U.) CEBAF-94-010 CATES, G.D. (Princeton U.) LAMPF-1231 CESTER, R. (Turin U.) FNAL-760 CESTER, R. (Turin U.) FNAL-835 CHANG, C.C.G. (Maryland U.) CEBAF-89-033 CHASMAN, C. (Brookhaven) BNL-866 CHASTELER, R. (Duke U.) CEBAF-93-036 CHATTERJEE, L. (Jadavpur U.) FNAL-802 CHIBA, J. (KEK, Tsukuba) KEK-257 CHRIEN, R.E. (Brookhaven) BNL-874 CHRIEN, R.E. (Brookhaven) BNL-890 CHRIEN, R.E. (Brookhaven) BNL-906 CHRIEN, R.E. (Brookhaven) CEBAF-89-009 CHRISTIAN, D.C. (Fermilab) FNAL-862 CHUNG, S.U. (Brookhaven) BNL-852 CLAJUS, M. (UCLA) SATURNE-258 CLEMENT, H. (Tubingen U.) PSI-R-85-13-3 CLEMENT, H. (Tubingen U.) PSI-R-89-03 COLE, B.A. (Columbia U.) BNL-910 COLE, P.L. (George Washington U.) CEBAF-94-109 COMFORT, J.R. (Arizona State U.) LAMPF-1178 COMFORT, J.R. (Arizona State U.) LAMPF-1256 CONETTI, S. (Virginia U.) FNAL-771 CONNELLY, J.P. (George Washington U.) CEBAF-94-109 COOPER, M.D. (Los Alamos) LAMPF-969 COOPER, M.D. (Los Alamos) LAMPF-1240 COOPER, P.S. (Fermilab) FNAL-781 CORCORAN, M.D. (Rice U.) FNAL-683 COUSINS, R.D. (UCLA) BNL-888 COX, B.B. (Virginia U.) FNAL-771 CRABB, D.G. (Virginia U.) CEBAF-91-023 CRAWFORD, H.J. (UC, Berkeley, Space Sci.) BNL-878 CRAWFORD, H.J. (UC, Berkeley, Space Sci.) BNL-896 CUMALAT, J.P. (Colorado U.) FNAL-687 CUMALAT, J.P. (Colorado U.) FNAL-831 CUMMING, J.B. (Brookhaven) BNL-844 CUMMINGS, W. (Simon Fraser U.) TRIUMF-683 DATZ, S. (Oak Ridge) CERN-WA-099 DATZ, S. (Oak Ridge) CERN-WA-099-2 DAUM, M. (PSI, Villigen) PSI-R-95-03 DAUM, M. (PSI, Villigen) PSI-R-96-05 DAVIS, C.A. (Manitoba U. and TRIUMF) BNL-885 DAVIS, C.A. (TRIUMF) TRIUMF-498 DAVIS, R., JR. (Penn U.) UNDERGR-HOMESTAKE-CHLORINE DAVISON, N.E. (Manitoba U.) TRIUMF-372

DAY, D.B. (Virginia U.) CEBAF-89-008

DAY, D.B. (Virginia U.) CEBAF-93-026

DE MARCO, N. (Turin U. and INFN, Turin) SATURNE-237 DE SANCTIS, E. (Frascati) CEBAF-93-017

DEHNHARD, D. (Minnesota U.) LAMPF-1267

DEMIDOV, V.S. (Moscow, ITEP) ITEP-921

DEMIDOV, V.S. (Moscow, ITEP) ITEP-922

DENISOV, S.P. (Serpukhov, IFVE) SERPUKHOV-152

DENNIS, L.C. (Florida State U.) CEBAF-89-043

DEUTSCH, M. (MIT) BNL-781

DI LELLA, L. (CERN) CERN-WA-096

DIDELEZ, J.P. (Orsay, IPN) SATURNE-121

DIDELEZ, J.P. (Orsay, IPN) SATURNE-209

DIEBOLD, G. (Yale U.) BNL-886 


\section{SPOKESPERSON INDEX}

DOLGOLENKO, A.G. (Moscow, ITEP) ITEP-913 DOMOGATSKY, G.V. (Moscow, INR) UNDERWAT-BAIKAL DUKES, E.C. (Virginia U.) FNAL-871

DYTMAN, S.A. (Pittsburgh U.) CEBAF-89-039

DZIERBA, A. (Indiana U.) CEBAF-94-016

EGGER, J.P. (Neuchatel U.) PSI-R-86-05

EICHLER, R.A. (Zurich, ETH) DESY-HERA-H1

ELOUADRHIRI, $\boldsymbol{L}$. (Christopher Newport U.) CEBAF-94-005

ELSEN, E. (DESY) DESY-HERA-H1

ENYO, H. (Kyoto U.) KEK-325

ERMOLOV, P.F. (Moscow State U.) SERPUKHOV-161

EVANS, D. (Birmingham U.) CERN-WA-085

EWAN, G.T. (Queens U., Kingston) UNDERGR-SUDBURY

FAESSLER, M.A. (Munich U.) CERN-PS-197

FETSCHER, W. (Zurich, ETH) PSI-R-94-10

FICENEC, J. (Virginia Tech) CEBAF-94-103

FILIPPONE, B.W. (Cal Tech) CEBAF-89-008

FILIPPONE, B.W. (Cal Tech) SLAC-NE-18

FINGER, M. (Charles U. and Dubna, JINR) PSI-R-95-08

FINN, J.M. (William and Mary Coll.) CEBAF-91-010

FLYAGIN, V.B. (Dubna, JINR) SERPUKHOV-167

FONTAINE, J.M. (Saclay) SATURNE-225

FRANKLIN, G.B. (Carnegie Mellon U.) BNL-813

FRANKLIN, G.B. (Carnegie Mellon U.) BNL-836

FRANKLIN, G.B. (Carnegie Mellon U.) BNL-885

FRANZINI, P. (Rome U.) FRASCATI-DAPHNE-KLOE

FRASCARIA, R. (Orsay, IPN) SATURNE-121

FRASCARIA, R. (Orsay, IPN) SATURNE-220

FRIEDMAN, E. (Hebrew U.) TRIUMF-598

FROSCH, R. (PSI, Villigen) PSI-R-87-01

FRULlaNI, S. (Rome U., Torvergata) CEBAF-91-011

FUKUDA, T. (Tokyo U., INS) BNL-906

FUNSTEN, H.O. (William and Mary Coll.) CEBAF-89-043

FUNSTEN, H.O. (William and Mary Coll.) CEBAF-91-024

FUNSTEN, H.O. (William and Mary Coll.) CEBAF-93-022

FURGET, C. (Grenoble U.) SATURNE-290

GAARDE, C. (Copenhagen U.) SATURNE-190

GABRIELSE, G. (Harvard U.) CERN-PS-196

GAGLIARDI, C. (Texas A and M) TRIUMF-614

GAI, M. (Connecticut U.) CEBAF-89-038

GAO, H. (Illinois U., Urbana) CEBAF-94-104

GARCON, M. (Saclay) SATURNE-258

GAVRIN, V.N. (Moscow, INR) UNDERGROUND-SAGE

GEER, S. (Fermilab) FNAL-861

GEER, S. (Fermilab) FNAL-868

GHOSH, D. (Jadavpur U.) CERN-EMU-014

GHOSH, D. (Jadavpur U.) FNAL-802

GIACOMELLI, G. (Bologna U.) CERN-EMU-018

GIACOMELLI, G. (Bologna U.) UNDERGR-MACRO

GILL, D.R. (TRIUMF) TRIUMF-614

GILMAN, R. (Rutgers U.) CEBAF-94-012

GIOVANETTI, K. (James Madison U.) CEBAF-89-039

GLAGOLEV, V.V. (Dubna, JINR) JINR-LHE-0941-5

GLASHAUSSER, C. (Rutgers U.) CEBAF-89-033

GLASS, G. (Texas U.) LAMPF-1309

GOLLIN, G.D. (Illinois U., Urbana) FNAL-773

GOMEZ, J. (CEBAF) CEBAF-93-024

GORRINGE, T.P. (Kentucky U.) TRIUMF-612

GOTTA, D. (Julich, Forschungszentrum) CERN-PS-207

GOTTA, D. (Julich, Forschungszentrum) PSI-R-94-01

GOULD, H. (LBL, Berkelcy) BNL-892

GRAM, P.A.M. (Los Alamos) LAMPF-1310

GRANNIS, P.D. (SUNY, Stony Brook) FNAL-740

GRECHKO, V.E. (Moscow, ITEP) ITEP-892

GRECHKO, V.E. (Moscow, ITEP) ITEP-897

GREENIAUS, L.G. (Alberta U.) TRIUMF-369

GRION, N. (Trieste U.) TRIUMF-624

GRION, N. ('Trieste U.) TRIUMF-653

GUTBROD, H.H. (Darmstadi, GSI) CERN-WA-098
HAEUSSER, O.F. (TRIUMF and Simon Fraser U.) LAMPF-1267

HAEUSSER, O.F. (TRIUMF and Simon Fraser U.) TRIUMF-557

HAEUSSER, O.F. (TRIUMF and Simon Fraser U.) TRIUMF-566

FAAFFTER, P. (Basel U.) PSI-Z-89-06

HAGUENAUER, M. (Ecole Polytech, LPNHE) CERN-UA-004-2

HALLMAN, T.J. (UCLA) BNL-896

HALZEN, F. (Wisconsin U., Madison) UNDERICE-AMANDA

HAMAGAKI, H. (Tokyo U., INS) BNL-866

HARSHMAN, D.R. (AT\&T Bell Labs, Murray Hill) BNL-849

HARTMANN, F.J. (Munich, Tech. U.) PSI-R-91-08

HASHIMOTO, O. ('Tokyo U., INS) KEK-140A

HASHIMOTO, O. (Tokyo U., INS) KEK-336

HASINOFF, M.D. (British Columbia U.) TRIUMF-452

HAUSSER, O.F. (Simon Fraser U. and TRIUMF) TRIUMF-683

HE, Y. (UC, Berkeley) CERN-WA-101

HEDDLE, D. (Christopher Newport U.) CEBAF-94-005

HEINRICH, W. (Siegen U.) CERN-EMU-017

HEPPELMANN, S. (Penn State U.) BNL-850

HERSMAN, F.W. (New Hampshire U.) JINR-LHE-0941-1C

HESS, R. (Geneva U.) SATURNE-144

HEUER, R.D. (CERN) CERN-LEP-OPAL

HICKS, K.H. (Ohio U.) BNL-887

HICKS, K.H. (Ohio U.) CEBAF-93-030

HICKS, K.H. (Ohio U.) TRIUMF-633

HILL, J.C. (Iowa State U.) CERN-NA-053

HOLT, R.J. (Illinois U., Urbana) CEBAF-89-012

HOLT, R.J. (Illinois U., Urbana) CEBAF-94-012

HOLT, R.J. (Illinois U., Urbana) CEBAF-94-104

HOLT, R.J. (Illinois U., Urbana) SLAC-NE-17

HOLZSCHEITER, M.H. (Los Alamos) CERN-PS-200

HOURANI, E. (Orsay, IPN) SATURNE-209

HSIUNG, Y.B. (Fermilab) FNAL-832

HUGHES, E.W. (SLAC) SLAC-E-142

HUGHES, E.W. (SLAC) SLAC-E-154

HUGHES, V.W. (Yale U.) BNL-821

HUGHES, V.W. (Yale U.) CERN-NA-047

HUGHES, V.W. (Yale U.) LAMPF-1054

HUNGERFORD, E.V. (Houston U.) BNL-774

HUNGERFORD, E.V. (Houston U.) BNL-874

HUNGERFORD, E.V. (Houston U.) BNL-907

HUNGERFORD, E.V. (Houston U.) CEBAF-89-009

HUSSEIN, A.H. (Northern British Columbia U.) LAMPF-1286

HUTCHEON, D.A. (TRIUMF) TRIUMF-643

HYDE-WRIGHT, C.E. (Old Dominion U.) CEBAF-91-014

IEIRI, M. (KEK, Tsukuba) KEK-251

IEIRI, M. (KEK, Tsukuba) KEK-289

IMAI, K. (Kyoto U.) BNL-886

IMAI, K. (Kyoto U.) KEK-224

IMAZATO, J. (KEK, Tsukuba) KEK-246

IVANOV, Y.M. (St. Petersburg, INP) SERPUKHOV-177

IWASA KI, M. (Tokyo U., INS) KEK-228

JACKSON, G.P. (Fermilab) FNAL-853

JACKSON, H.E. (Argonne) CEBAF-91-003

JAHN, R. (Bonn U.) SATURNE-222

JAIN, P.L. (SUNY, Buffalo) BNL-875

JAIN, P.L. (SUNY, Buffalo) CERN-EMU-011

JANOUSCH, M. (Zurich, ETH) PSI-R-95-09

JASTRZEBSKI, J. (Warsaw U., Heavy Ion Lab) CERN-PS-209

JELLEY, N.A. (Oxford U.) UNDERGROUND-SUDBURY

JENKINS, D.A. (Virginia Tech) CEBAF-94-103

JOHNS, K.A. (Arizona U.) FNAL-800

JOHNSON, R.R. (British Columbia U.) TRIUMF-624

JOURDAN, J. (Basel U.) PSI-Z-89-02

JULLIAN, S. (Orsay, LAL) NEMO-2 (listed under ITEP)

JUNGMANN, K. (Ileidelberg U., Phys. Inst.) PSI-R-89-06

JUNGMANN, K. (Heidelberg U., Phys. Inst.) PSI-R-92-08

KAJIKAWA, R. (Nagoya U.) KEK-TE-002

KALBFLEISCH, G.R. (Oklahoma U.) FNAL-855

KANAVETS, V.P. (Moscow, I'TEP) ITEP-914 


\section{SPOKESPERSON INDEX}

KANE, J.R. (William and Mary Coll.) 8NL-871 KAPLAN, D.M. (Northern Inlinois U.) FNAL-789 KAWABATA, S. (KEK, Tsukuba) KEK-TE-002 KAWAI, H. (Chiba U.) KEK-248

KEKELIDZE, V.D. (Dubna, JINR) SERPUKHOV-159 KHACHATUROV, B.A. (Dubna, JINR) JINR-LHE-0941-4 KHACHATUROV, B.A. (Dubna, JINR) SATURNE-225 KHAN, H.A. (Pinstech, Islamabad) CERN-EMU-019 KHAN, H.A. (Pinstech, Islamabad) CERN-EMU-020 KHAZIN, B.I. (Novosibirsk, IYF) NOVOSIBIRSK-CMD-2 KHRYKIN, A.S. (Dubna, JINR) JINR-LNP-09 KILIAN, K. (Julich, Forschungszentrum) CERN-PS-185-2 KIM, C.O. (Korea U.) FNAL-843

KINSON, J.B. (Birmingham U.) CERN-WA-094

KIRK, A. (CERN) CERN-WA-091

KIRK, A. (CERN) CERN-WA-102

KIRPICHNIKOV, I.V. (Moscow, ITEP) ITEP-861

KIRPICHNIKOV, I.V. (Moscow, ITEP) ITEP-912 KIRPICHNIKOV, I.V. (Moscow, ITEP) ITEP-921 KIRSTEN, T. (Heidelberg, MPI) UNDERGR-GALLEX KISELEV, Y.T. (Moscow, ITEP) ITEP-873 KISELEV, Y.T. (Moscow, ITEP) ITEP-911 KISELEV, Y.T. (Moscow, ITEP) ITEP-941 KISELEV, Y.T. (Moscow, ITEP) ITEP-951 KISHIMOTO, T. (Osaka U.) KEK-278 KLANNER, R. (DESY) DESY-HERA-ZEUS KLEIN, S.R. (LBL, Berkeley) SLAC-E-146 KLUBERG, . . (Ecole Polytechnique, LPNHE) CERN-NA-038 KLUBERG, L. (Ecole Polytechnique, LPNHE) CERN-NA-050 KLUBERG, L. (Ecole Polytechnique, LPNHE) CERN-NA-051 KLUGE, W. (Karlsruhe U., IEKP) PSI-R-85-13-3 KNAPP, B.C. (Columbia U.) FNAL-690 KORKMAZ, E. (Northern British Columbia U.) TRIUMF-704 KOSSOV, M.V. (Moscow, ITEP) CEBAF-93-012 KOSSOV, M.V. (Moscow, ITEP) CEBAF-94-002. KOSSOV, M.V. (Moscow, ITEP) ITEP-853 KOTELNIKOV, K.A. (Lebedev Inst.) CERN-EMU-015 KOVASH, M.A. (Kentucky U.) KEK-235

KOVASH, M.A. (Kentucky U.) TRIUMF-643

KOVASH, M.A. (Kentucky U.) TRIUMF-661

KOX, S. (Grenoble U.) SATURNE-166

KOX, S. (Grenoble U.) SATURNE-235

KOX, S. (Grenoble U.) SATURNE-253

KOX, S. (Grenoble U.) SATURNE-290

KRISCH, A.D. (Michigan U.) SERPUKHOV-UNK-001 KRUGLOV, S.P. (St. Petersburg, INP) BNL-913-914 KRUGLOV, S.P. (St. Petersburg, INP) ITEP-914 KRUGLOV, S.P. (St. Petersburg, INP) PNPI-SC-124 KRUTENKOVA, A.P. (Moscow, ITEP) ITEP-901 KRUTENKOVA, A.P. (Moscow, ITEP) ITEP-923 KRYSHKIN, V.I. (Serpukhov, IFVE) SERPUKHOV-155 KRYSHKIN, V.I. (Serpukhov, IFVE) SERPUKHOV-175 KUHN, S.E. (Old Dominion U.) CEBAF-93-009 KUNNE, R.A. (Saclay) SATURNE-278 KUREPIN, A.B. (Moscow, INR) JINR-LHE-0969 KWIATKOWSKI, K. (Indiana U.) BNL-900 KYCIA, T. (Brookhaven) BNL-913-914 LAGET, J.M. (Saclay) CEBAF-93-031

LANDE, K. (Penn U.) LAMPF-1213

LANDE, K. (Penn U.) UNDERGR-HOMESTAKE-CHLORINE LANDE, K. (Penn U.) UNDERGR-HOMESTAKE-IODINE LANDSBERG, L.G. (Serpukhov, IFVE) SERPUKHOV-169 LANDSBERG, L.G. (Serpukhov, IFVE) SERPUKHOV-178 LARSON, B. (Simon Fraser U.) TRIUMF-557

LE BORNEC, Y. (Orsay, IPN) SATURNE-186 LE BORNEC, Y. (Orsay, IPN) SATURNE-280 LEARNED, J.G. (Hawaii U.) UNDERWATER-DUMAND LEDOUX, R.J. (MIT) BNL-859

LEHAR, F. (Saclay) JINR-LHE-0941-4

LEHAR, F. (Saclay) SATURNE-144
LEKSIN, G.A. (Moscow, ITEP) ITEP-853 LEKSIN, G.A. (Moscow, ITEP) ITEP-894 LEKSIN, G.A. (Moscow, ITEP) ITEP-895 LEPIKHIN, Y.B. (Moscow, ITEP) ITEP-865

LI, J. (Beijing, Inst. High Energy Phys.) BEPC-BES LINDENBAUM, S.J. (BNL and City Coll., N.Y.) BNL-810 LINDENBAUM, S.J. (BNL and City Coll., N.Y.) BNL-881 LITTENBERG, L.S. (Brookhaven) BNL-787 LITVINENKO, A. (Dubna, JINR) JINR-LHE-0983-4 LOPATIN, I.V. (St. Petersburg, INP) PNPI-SC-147 LORD, J.J. (Washington U., Seattle) FNAL-793 LOUIS, W.C. (Los Alamos) LAMPF-1173 LOURIE, R. (Virginia U.) CEBAF-91-011 LUK, K.B. (LBL, Berkeley and UC, Berkeley) FNAL-871 LUNDBERG, B.G. (Fermilab) FNAL-872 LYTKIN, L. (Dubna, JINR) SATURNE-258 LYTKIN, L. (Dubna, JINR) SATURNE-280 LYUBIMOV, V.A. (Moscow, ITEP) ITEP-832 MACK, D.J. (CEBAF) CEBAF-93-021

MACRI, M. (Genoa U. and INFN, Genoa) CERN-PS-202

MADEY, R. (Hampton U.) CEBAF-93-038

MAGAHIZ, R. (Carnegie Mellon U.) CEBAF-95-003 MAJKA, R.D. (Yale U.) BNL-864

MALAKHOV, A.X. (Dubna, JINR) JINR-LHE-0971-1 MALAKHOV, A.x. (Dubna, JINR) JINR-LHE-0971-2 MANLEY, D.M. (Kent State U.) CEBAF-91-024 MARCHAND, C. (Saclay) CEBAF-93-031 MARSHAK, M.L. (Minnesota U.) UNDERGROUND-SOUDAN-2 MARTELLOTTI, G. (Rome U. and INFN, Rome) CERN-WA-084 MARTINSKA, G. (Kosice U.) JINR-LHE-0941-5 MASCARENHAS, N. (Cal Tech) CERN-NA-055 MASSON, G.S. (Basel U.) PSI-Z-89-07

MASUDA, Y. (KEK, Tsukuba) KEK-231 MATHYE, E.L. (Regina U.) TRIUMF-506 MATSUI, T. (KEK, Tsukuba) KEK-TE-001

MATthiAs, B. (Heidelberg U., Phys. Inst.) PSI-R-92-08 MAY, M. (Brookhaven) BNL-781

MAY, M. (Brookhaven) BNL-885

MAYER, B. (Saclay) SATURNE-198

MAYER, B. (Saclay) SATURNE-246

MAYTAL-BECK, S. (Tel Aviv U.) TRIUMF-445

MCCARTHY, J.S. (Virginia U.) SLAC-E-155

MCDONALD, A.B. (Queens U., Kingston) UNDERGR-SUDBURY MCDONALD, K.T. (Princeton U.) SLAC-E-144

MCGAUGHEY, P.L. (Los Alamos) FNAL-866

MCNAUGHTON, M.W. (Los Alamos) LAMPF-876

MCNAUGHTON, M.W. (Los Alamos) LAMPF-1234

MCNAUGHTON, M.W. (Los Alamos) LAMPF-1293

MEASDAY, D.F. (British Columbia U.) TRIUMF-537

MECKING, B.A. (CEBAF) CEBAF-89-045

MECKING, B.A. (CEBAF) CEBAF-91-024

MELISSINOS, A.C. (Rochester U.) BNL-840

MELISSINOS, A.C. (Rochester U.) SLAC-E-144

MESTAYER, M. (CEBAF) CEBAF-93-030

MEZIANI, Z.-E. (Temple U.) CEBAF-94-010

MIGNEREY, A. (Maryland U.) BNL-917

MILLS, A.P., JR. (A'\&'T Bell Labs, Murray Hill) BNL-849

MILNER, R.G. (MI'T, LNS) CEBAF-91-007

MILNER, R.G. (MIT, LNS) DESY-HERA-HERMES

MILNER, R.G. (MIT, LNS) SLAC-NE-18

MINEHART, R.C. (Virginia U.) CEBAF-89-037 MINEHART, R.C. (Virginia U.) CEBAF-89-038 MINEHART, R.C. (Virginia U.) CEBAF-89-042 MINEHART, R.C. (Virginia U.) CEBAF-91-023 MINEHART, R.C. (Virginia U.) CEBAF-93-036 MISCHKE, R.E. (Los Alamos) LAMPF-1240

MISKIMEN, R.A. (Massachusetts U., Amherst) CEBAF-94-015 MOISEEV, A.M. (Serpukhov, IFVE) SERPUKHOV-161

MOLZON, W.R. (UC, Irvine) BNL-871

MONTGOMERY, H.E. (Fermilab) FNAL-740 


\section{SPOKESPERSON INDEX}

MORAlES, A. (Zaragoza U.) ITEP-912 MORLET, M. (Orsay, IPN) SATURNE-253 MORONI, L. (INFN, Milan) FNAL-831 MORRIS, C.L. (Los Alamos) LAMPF-1190 MORSCH, H.P. (Saclay) SATURNE-220 MORSCH, H.P. (Saclay) SATURNE-251 MORSE, W.M. (Brookhaven) BNL-821 MURPHY, C.T. (Fermilab) FNAL-853 MUTCHLER, G.S. (Rice U.) CEBAF-89-024 NAGAE, T. (Tokyo U., INS) BNL-905 NANDA, S. (CEBAF) CEBAF-89-033 NAPOLITANO, J.J. (Rensselaer Poly) CEBAF-93-033 NAPOLITANO, J.J. (Rensselaer Poly) CEBAF-94-014 NAPOLITANO, J.J. (Rensselaer Poly) CEBAF-94-016 NARASIMHAM, V.S. (Tata Inst.) UNDERGROUND-KGF NAVILIAT-CUNCrC, O. (Zurich, ETH) PSI-Z-90-07 NEFKENS, B.M.K. (UCLA) BNL-890 NEFKENS, B.M.K. (UCLA) BNL-913-914 NEFKENS, B.M.K. (UCLA) SATURNE-198 NEFKENS, B.M.K. (UCLA) SATURNE-246 NEMENOV, L.L. (Dubna, JINR) CERN-PS-212 NEMENOV, L.L. (Dubna, IINR) SERPUKHOV-174 NIETO, M.M. (Los Alamos) CERN-PS-200 NIORADZE, M.S. (Tbilisi State U.) JINR-L.HE-0941-5 NOLTE, E. (Munich, Tech U.) CERN-NA-054 NOZDRACHEV, V.N. (Moscow, ITEP) SERPUKHOV-173 NUMAO, T. (TRIUMF) TRIUMF-703

OELERT, W. (Julich, Forschungszentrum) CERN-PS-210

OLSEN, S.L. (Hawaii U.) KEK-TE-003

OPPER, A.K. (Alberta U.) TRIUMF-704

OREAR, J. (Cornell U., LNS) FNAL-811

OTTERLUND, X. (Lund U.) BNL-863

OTTERLUND, I. (Lund U.) CERN-EMU-012

PAGE, S.A. (Manitoba U.) TRIUMF-497-287

PANEBRATSEV, Y.A. (Dubna, JINR) JINR-LHE-1011-1

PAOLONE, V. (UC, Davis) FNAL-872

PAUL, S. (Heidelberg, Max Planck Inst.) CERN-WA-089

PAVAN, M.M. (British Columbia U.) TRIUMF-645

PAVLOPOULOS, P. (Basel U.) CERN-PS-195

PENG, J.C. (Los Alamos) 8NL-890

PENG, J.C. (Los Alamos) BNL-907

PENG, J.C. (Los Alamos) FNAL-789

PERDRISAT, C.F. (William \& Mary Colf.) CEBAF-93-027 PERDRISAT, C.F. (William \& Mary Coll.) SATURNE-202 PERDRISAT, C.F. (William \& Mary Coll.) SATURNE-249 PERROT, F. (Saclay) SATURNE-144

PETERSON, R.J. (Colorado U.) BNL-874 PETERSON, R.J. (Colorado U.) KEK-352 PETRATOS, G.M.G. (Kent State U.) CEBAF-91-026 PETRatos, G.M.G. (Kent State U.) CEBAF-93-024 PIILONEN, L.E. (Virginia Tech) LAMPF-1240

PILE, P.H. (Brookhaven) BNL-886

PINFOLD, J.L. (Alberta U.) CERN-LEP-06

PISKUNOV, N.M. (Dubna, JINR) JINR-LHE-0941-1A to -ID PISKUNOV, N.M. (Dubna, JINR) JINR-LHE-0941-4

PLATNER, E.D. (Brookhaven) BNL-810

PLATNER, E.D. (Brookhaven) BNL-891

POĆANIĆ, D. (Virginia U.) LAMPF-1179

POC̈ANIĆ, D. (Virginia U.) PSI-R-89-01

POLING, R.A. (Minnesota U.) CESR-CLEO

PREEDOM, B. (South Carolina U.) CEBAF-94-002

PREEDOM, B. (South Carolina U.) SATURNE-121

PRETZL, K. (Bern U.) CERN-NA-052

PRETZL, K. (Bern U.) PSI-Z-90-12

PRICE, P.B. (UC, Berkeley) BNL-882

PROKOFIEV, A.N. (St. Petersburg, INP) PNPI-SC-129

PROKOSHKIN, Y.D. (Serpukhov, IFVE) CERN-NA-012-2

PROKOSHKIN, Y.D. (Serpukhov, IFVE) SERPUKHOV-172

PUNJABI, V. (Norfolk State U.) CEBAF-93-027

PUNJABI, V. (Norfolk State U.) SATURNE-249
PUROHIT, M.V. (South Carolina U.) FNAL-791 QUERCIGH, E. (CERN) CERN-WA-097 RAGAZZI, S. (Milan U. and INFN, Milan) CERN-NA-056

RAI, G. (LBL, Berkeley) BNL-895

RAMEIKA, R.A. (Fermilab) FNAL-800

RAPIN, D. (Geneva U.) SATURNE-225

REAY, N.W. (Kansas State U.) FNAL-803

REBKA, G.A., JR. (Wyoming U.) LAMPF-1310

REKALO, M.P. (Kharkov, FTI) JINR-LHE-0941-3

REMSBERG, L.P. (Brookhaven) BNL-859

RIPANI, M. (INFN, Genoa) CEBAF-93-006

RISTINEN, R.A. (Colorado U.) LAMPF-1190

RITCHIE, B.G. (Arizona State U.) CEBAF-91-008

RITCHIE, B.G. (Arizona State U.) CEBAF-94-008

RITCHIE, J.L. (Texas U.) BNL-871

ROBERTS, B.L. (Boston U.) BNL-821

ROBERTS, D.A. (Michigan U.) LAMPF-1310

ROBERTS, J.B. (Rice U.) BNL-817

ROCK, S.E. (American U.) SLAC-E-140X

ROEHRICH, K. (Julich, Forschungszentrum) CERN-PS-185-2 ROEHRICH, K. (Julich, Forschungszentrum) CERN-PS-185-3 ROLANDI, L. (CERN) CERN-LEP-ALEPH RONDON, O.A. (Virginia U.) SLAC-E-143

ROSSI, L. (Gcnoa U. and INFN, Genoa) CERN-WA-092 ROSSI, P. (Frascati) CEBAF-93-017

ROTONDI, A. (Pavia U. and INFN, Pavia) CERN-PS-201 RUBBIA, C. (CERN) CERN-PS-211 RUBBIA, C. (CERN) UNDERGROUND-ICARUS RUBIN, P.D. (Richmond U.) CEBAF-93-022 RUY, R. (Trieste U.) TRIUMF-508

R.UI, R. (Tricste U.) TRIUMF-624

RUI, R. (Trieste U.) TRIUMF-653

RUSS, J.S. (Carnegie Mellon U.) FNAL-781

SADLER, M.E. (Abilene Christian U.) BNL-913-914

SADLER, M.E. (Abilene Christian U.) LAMPF-1268

SAKAGUCHI, H. (Kyoto U.) KEK-269

SANDWEISS, J. (Yale U.) BNL-864

SARYCHEVA, L.X. (Moscow State U.) JINR-LHE-0971-1

SARYCHEVA, L.I. (Moscow State U.) JINR-LHE-0971-2

SASAO, N. (Kyoto U.) KEK-162

SAWAFTA, R. (Brookhaven) BNL-887

SCHELLMAN, H.M. (Northwestern U.) FNAL-665

SCHMITT, H. (Freiburg U.) PSI-R-87-12

SCHMITT, H. (Freiburg U.) PSI-R-95-08

SCHROEDER, H. (DESY) DESY-DORIS-ARGUS

SCHUMACHER, R.A. (Carnegie Mellon U.) CEBAF-89-004

SCHWARTZ, A.J. (Princeton U.) BNL-888

SEALOCK, R. (Virginia U.) CEBAF-89-017

SELIVANOV, V. (Kurchatov Inst., Moscow) TRIUMF-614

SEREDNYAKOV, S.I. (Novosibirsk, IYF) NOVOSIBIRSK-SND

SETO, R.K. (UC, Riverside) BNL-917

SEVIOR, M.E. (British Columbia U.) TRIUMF-561

SEVIOR, M.E. (British Columbia U.) TRIUMF-624

SEYBOTH, P. (Munich, Max Planck Inst.) CERN-NA-035

SHAEVITZ, M.H. (Columbia U.) FNAL-815

SHAROV, V.I. (Dubna, JINR) JINR-LHE-0941-4

SHEINKMAN, V.A. (Moscow, ITEP) ITEP-911

SHEINKMAN, V.A. (Moscow, I'TEP) ITEP-941

SHEINKMAN, V.A. (Moscow, ITEP) ITEP-951

SHIMANSKIY, S.S. (Dubna, JINR) JINR-LHE-1011-1

SHINOHARA, A. (Nagoya U.) KEK-262

SIEBERT, R. (Orsay, IPN) SATURNE-222

SIEMIARCZUK, T. (Warsaw, INR) JINR-LHE-0941-5

SITNIK, I.M. (Dubna, JINR) JINR-LHE-0941-1A and -1D

SITNIK, I.M. (Dubna, JINR) JINR-LHE-0941-3

SITNIK, I.M. (Dubna, JINR) SATURNE-249

SLATTERY, P.F. (Rochester U.) FNAL-706

SMIRNITSKY, A.V. (Moscow, ITEP) ITEP-895

SMITH, A.J.S. (Princeton U.) BNL-787

SMITH, E. (CEBAF) CEBAF-93-022 


\section{SPOKESPERSON INDEX}

SMITH, G.R. (TRIUMF) TRIUMF-560

SMITH, G.R. (TRIUMF) TRIUMF-719

SOBER, D.I. (Catholic U.) CEBAF-91-015

SOKOLOVSKY, V.V. (MOSCOW, ITEP) SERPUKHOV-147

SOLOVIANOV, V.L. (Serpukhov, IFVE) SERPUKHOV-UNK

SOMOV, S.V. (Moscow Phys. Eng. Inst.) SERPUKHOV-128

SOUDER, P.A. (Syracuse U.) CEBAF-91-010

SOUDER, P.A. (Syracuse U.) LAMPF-1231

SPINKA, H. (Argonne) BNL-913-914

SPINKA, H. (Argonne) JINR-LHE-0941-4

SPINKA, H. (Argonne) SATURNE-225

STEADMAN, S.G. (MIT) 8NL-866

STEVENSON, N.R. (Saskatchewan U.) TRIUMF-502

STOCK, R. (Frankfurt U.) CERN-NA-049

STOLER, P. (Rensselaer Poly) CEBAF-91-002

STROKOVSKY, E.A. (Dubna) JINR-LHE-0941-1A and -1B

STROKOVSKY, E.A. (Dubna) SATURNE-278

STROOT, J.P. (Brussels U., IISN) CERN-NA-012-2

STRUNOV, L.N. (Dubna, JINR) JINR-LHE-0941-1E

STRUNOV, L.N. (Dubna, JINR) JINR-LHE-0941-4

SUTORMIN, A.I. (Moscow, ITEP) ITEP-942

TAIUTI, M. (INFN, Genoa) CEBAF-91-002

TAKAHASHI, Y. (Alabama U., Huntsvilie) CERN-EMU-016

TAMURA, H. (Tokyo U.) KEK-218

TAMURA, H. (Tokyo U.) KEK-287

TAMURA, H. (Tokyo U.) KEK-326

TANNER, N.W. (Oxford U.) UNDERGROUND-SUDBURY

TAQQU, D. (PSI, Villigen) PSI-R-91-08

TATISCHEFF, B. (Orsay, IPN) SATURNE-244

TAYLOR, C.C. (Case Western Reserve U.) FNAL-864

TEREKHOV, Y.V. (Moscow, ITEP) ITEP-873

THIBAULT, C. (Orsay, CSNSM) CERN-PS-189

TIKHONOV, Y.A. (Novosibirsk, IYF) NOVOSIBIRSK-KEDR

TING, S.C.C. (MIT) CERN-LEP-L3

TIPPENS, W.B. (UCLA) BNL-909

TIPPENS, W.B. (UCLA) BNL-913-914

TOKI, W. (Colorado State U.) BEPC-BES

TOMASI-GUSTAFSSON, E. (Saclay) SATURNE-253

TOTSUKA, Y. (Tokyo U., ICRR) UNDERGR-KAMIOK.

TOTSUKA, Y. (TOKyo U., ICRR) UNDERGR-SUPERKAMIOK.

TREILLE, D. (CERN) CERN-LEP-DELPHI

TRIBBLE, $R$. (Texas $A$ and $M$ ) TRIUMF-614

TROYAN, Y.A. (Dubna, JINR) JINR-LHE-0936-3

TSERRUYA, I. (Weizmann Inst.) CERN-NA-045

TSERRUYA, X. (Weizmann Inst.) CERN-NA-045-2

TYAPKIN, A.A. (Dubna, JINR) SERPUKHOV-157

UGGERHOJ, E. (Aarhus U.) CERN-NA-043-2

UGGERHOJ, E. (Aarhus U.) CERN-PS-194-3

USOV, Y.A. (Dubna, JINR) JINR-LNP-23

VAN DER SCHAAF, A. (Zurich U.) PSI-R-87-03

VAN OERS, W.T.H. (Manitoba U.) TRIUMF-369

VAN OERS, W.T.H. (Manitoba U.) TRIUMF-497-287
VAN ORDEN, J.W. (CEBAF) CEBAF-89-033

VASILIEV, A.N. (Serpukhov, IFVE) SERPUKHOV-149

VENUS, W. (Rutherford) CERN-LEP-DELPHI

VERCELLIN, E. (Turin U.) SATURNE-237

VINEYARD, M.F. (Richmond U.) CEBAF-93-008

VINEYARD, M.F. (Richmond U.) CEBAF-94-017

VIOLA, V.E. (Indiana U.) BNL-900

VLASSOV, A.V. (Moscow, ITEP) ITEP-894

VODOPIANOV, A.S. (Dubna, JINR) JINR-LHE-1010

VON EGIDY, T. (Munich, Tech. U.) CERN-PS-203

VOVENKO, A.S. (Serpukhov, IFVE) SERPUKHOV-136

WADDINGTON, C.J. (Minnesota U.) BNL-868

WADDINGTON, C.J. (Minnesota U.) BNL-869

WAH, Y.W. (Chicago U.) FNAL-799

WANG, K. (Virginia U.) CEBAF-94-015

WELLER, H.R. (Duke U.) CEBAF-93-036

WENDER, S.A. (Los Alamos) LAMPF-1208

WEYER, G. (Aarhus U.) CERN-IS-300

WHITNEY, R.R. (CEBAF) CEBAF-94-109

WHITTAL, D.M. (Simon Fraser U.) TRIUMF-630

WILDENHAIN, P. (Penn U.) UNDERGR-HOMESTAKE-IODINE WILHELM, I. (Charles U.) JINR-LNP-23

WILKES, R.J. (Washington U., Seattle) BNL-863

WILLIS, N. (Orsay, IPN) SATURNE-280

WINSTEIN, B.D. (Chicago U.) FNAL-832

WINTER, K. (Humboldt U., Berlin) CERN-WA-079

WINTER, K. (Humboldt U., Berlin) CERN-WA-095

WOJCICKI, S.G. (Stanford U.) BNL-871

WOJCICKI, S.G. (Stanford U.) FNAL-875

WOLTER, W. (Cracow, INP-Exp) CERN-EMU-013

WRIGHT, D.H. (TRIUMF) TRIUMF-592

WURZINGER, R. (Saclay) SATURNE-280

YAMANAKA, T. (Osaka U.) FNAL-799

YAMAZAKI, T. (Tokyo U., INS) CERN-PS-205

YEGNESWARAN, A.S. (CEBAF) CEBAF-94-015

YONNET, $J$. (Saclay) SATURNE-202

ZAITSEV, A.M. (Serpukhov, TFVE) SERPUKHOV-164

ZAJC, W.A. (Columbia U.) BNL-859

ZARUBIN, A.V. (Dubna, JINR) JINR-LHE-0941-1E

ZAVATTINI, E. (Trieste U.) PSI-R-93-06

ZEIDMAN, B. (Argonne) CEBAF-91-016

ZELDOVICH, O.Y. (Moscow, ITEP) ITEP-832

ZELLER, M.E. (Yale U.) BNL-865

ZGHICHE, A. (Strasbourg, CRN) SATURNE-280

ZICHICHI, A. (Bologna U.) UNDERGROUND-LVD

ZIEMINSKI, A. (Indiana U.) FNAL-672A

ZIHLMANN, B. (Basel U.) PSI-Z-91-02

ZOLIN, L. (Dubna, JINR) JINR-LHE-0983-4

ZU PUTLITZ, G. (Heidelberg U., Phys. Inst.) LAMPF-1054

ZUPRANSKI, P. (Soltan Inst.) SATURNE-190 


\section{ABBREVIATIONS USED IN THE SUMMARIES}

\section{JOURNALS}

Following are abbreviations for journals listed in the summaries:

\begin{tabular}{|c|c|}
\hline APL & Applied Physics Letters \\
\hline APP & Acta Physica Polonica \\
\hline AOPT & Applied Optics \\
\hline ASPP & Astroparticle Physics \\
\hline ASTJ & Astrophysical Journal \\
\hline CPC & Computer Physics Communications \\
\hline DANS & Doklady Akademii Nauk SSSR (in Russian) \\
\hline ECHAYA & Fizika Elementarnykh Chastits i Atomnogo Yadra (translated as PPN) \\
\hline EPL & Europhysics Letters \\
\hline FBS & Few Body Systems \\
\hline HEPNP & High Energy Physics and Nuclear Physics (in Chinese) \\
\hline HFI & Hyperfine Interactions \\
\hline IEEE TNS & Institute of Electrical and Electronics Engineers, Transactions on Nuclear Science \\
\hline IJMP & International Journal of Modern Physics \\
\hline JDEP & Journale de Physique \\
\hline JETPL & Journal of Experimental and Theoretical Physics Letters (English translation of ZETFP) \\
\hline JOSA & Journal of the Optical Society of America \\
\hline JPHY & Journal of Physics \\
\hline JPSJ & Journal of the Physical Society of Japan \\
\hline LNC & Lettere al Nuovo Cimento \\
\hline MPL & Modern Physics Letters \\
\hline NATU & Nature \\
\hline NC & Nuovo Cimento \\
\hline NIM & Nuclear Instruments and Methods \\
\hline NP & Nuclear Physics \\
\hline PAN & Physics of Atomic Nuclei (English translation of $\mathrm{YF}$ ) \\
\hline PHYU & Physics - Uspekhi (English translation of USP) \\
\hline PL & Physics Letters \\
\hline $\begin{array}{l}\text { PPN } \\
\text { PR }\end{array}$ & Physics of Particles and Nuclei (English translation of ECHAYA) \\
\hline $\begin{array}{l}\text { PR } \\
\text { PRI }\end{array}$ & Physical Review \\
\hline $\begin{array}{l}\text { PRL } \\
\text { PRPL }\end{array}$ & $\begin{array}{l}\text { Physical Review Letters } \\
\text { Physics Reports }\end{array}$ \\
\hline PS & Physica Scripta \\
\hline PTE & Pribory i Tekhnika Eksperimenta (in Russian) \\
\hline PTP & Progress of Theoretical Physics \\
\hline PW & Particle World \\
\hline RMP & Reviews of Modern Physics \\
\hline RPP & Reports on Progress in Physics \\
\hline RSI & Review of Scientific Instruments \\
\hline $\mathrm{SCI}$ & Science \\
\hline SJNP & \\
\hline USP & $\begin{array}{l}\text { Uspekhi Fizicheskikh Nauk (translated as PHYU) } \\
\text { Yadernaya Fizika (translated as SJNP before } 1993 \text {, and as PAN thereafter) }\end{array}$ \\
\hline $\begin{array}{l}\text { YE } \\
\text { ZETFP }\end{array}$ & $\begin{array}{l}\text { Yadernaya Fizika (translated as SJNP betore 1993, and as PAN inerealer) } \\
\text { Pisma v Zhurnal Eksperimentalnoi i Teoreticheskoi Fiziki (translated as JETPL) }\end{array}$ \\
\hline ZPHY & Zeitschrift für Physik \\
\hline
\end{tabular}




\section{KINEMATIC VARIABLES}

Following are abbreviations used with reactions to indicate the momenta or energies at which they are studied:

$\begin{array}{ll}\text { PLAB } & \text { beam momentum in the lab frame } \\ \text { TLAB } & \text { beam kinetic energy in the lab frame } \\ \text { ELAB } & \text { beam total energy in the lab frame } \\ \text { PLAB/N } & \text { beam momentum per nucleon in the lab frame } \\ \text { TLAB/N } & \text { beam kinetic energy per nucleon in the lab frame } \\ \text { ELAB/N } & \text { beam total energy per nucleon in the lab frame } \\ \text { ECM } & \text { total energy in the c.m. frame }\end{array}$

total energy in the c.m. frame

\section{ACCELERATORS}

BEPC
BNL
BNL-RHIC
CEBAF
CERN-LEAR
CERN-LEP
CERN-LHC
CERN-PBAR/P
CERN-SPS
CESR
DESY-DORIS-III
DESY-HERA
FNAL-COLLIDER
FNAL-TEV
FRASCATI-DAPHNE
ITEP
JINR
KEK
KEK-B-FACTORY
KEK-TRISTAN
LAMPF
NOVO-VEPP-2M
NOVO-VEPP-4M
PNPI
PSI
SATURNE-II
SERPUKHOV
SERPUKHOV-UNK
SLAC
SLAC-PEP2
SLAC-SLC
TRIUMF

Beijing $e^{+} e^{-}$collider ( $3.6 \mathrm{GeV} \mathrm{Ecm}$ )

Brookhaven AGS proton synchrotron ( $31 \mathrm{GeV} / \mathrm{c}$ Plab)

Brookhaven relativistic heavy ion collider $(100 \mathrm{GeV} / \mathrm{n}$ per beam)

CEBAF linear accelerator with continuous $e^{-}$beam $(4.0 \mathrm{GeV} \mathrm{Ecm})$

CERN Low-Energy Antiproton Ring

CERN Large Electron-Positron collider (90-180 GeV Ecm)

CERN Large Hadron Collider (14 TeV Ecm)

CERN $\bar{p} p$ collider ( $900 \mathrm{GeV}$ Ecm)

CERN Super Proton Synchroton $(450 \mathrm{GeV} / c$ Plab)

Cornell Electron-positron Storage Ring ( $16 \mathrm{GeV} \mathrm{Ecm)}$

DESY DORIS electron-positron double ring, 1991 upgrade

DESY HERA electron $(27 \mathrm{GeV})$ - proton $(820 \mathrm{GeV})$ collider

FNAL $\bar{p} p$ collider $(2000 \mathrm{GeV}$ Ecm)

FNAL fix target Tevatron $(1000 \mathrm{GeV})$

$e^{+} e^{-}$storage ring and $\phi$ factory $(1 \mathrm{GeV} \mathrm{Ecm})$

ITEP Moscow proton synchrotron ( $7 \mathrm{GeV} / c \mathrm{Plab})$

JYNR (Dubna) proton synchrotron $(10 \mathrm{GeV} / \mathrm{c} \mathrm{Plab})$

$\mathrm{KEK}$ proton synchrotron $(12 \mathrm{GeV} / \mathrm{c}$ Plab)

KEK two-ring asymmetric $\mathrm{B}$ factory

KEK electron-positron storage ring $(60 \mathrm{GeV} \mathrm{Ecm})$

Los Alamos Meson/Proton Factory (1460 MeV/c Plab)

Novosibirsk VEPP-2M electron-positron storage ring (1.4 GeV Ecm)

Novosibirsk VEPP-4M electron-positron storage ring ( $7 \mathrm{GeV} \mathrm{Ecm}$ )

Synchrocyciotron at St. Petersburg Inst. Nucl. Phys. (1 GeV Tlab)

Cyclotron at Paul Scherrer Institute (590 MeV Tlab)

Saclay Saturne-II $p, d$, and He synchrotron

Serpukhov proton synchrotron $(76 \mathrm{GeV} / c$ Plab)

Serpukhov multi-TeV proton machine

Stanford electron linear accelerator $(40 \mathrm{GeV} / \mathrm{c}$ Plab)

New SLAC Positron-Electron Project (asymmetric B factory)

SLAC Linear $e^{+} e^{-}$Collider (100 GeV Ecm)

Canadian TRIangle University Meson Facility (520 MeV Tlab) 


\section{DETECTORS}

In searching the EXPERIMENTS database (SLAC/SPIRES) from which this report is taken, use the following abbreviations for general kinds of detectors:

$\begin{array}{ll}\text { CALO } & \text { calorimeter } \\ \text { CNTR } & \text { counter(s) } \\ \text { COMB } & \text { combination of various elements } \\ \text { DAS } & \text { double-arm spectrometer } \\ \text { DRIFT } & \text { drift chamber } \\ \text { EMUL } & \text { emulsion } \\ \text { IONIZATION } & \text { detector looking for ionization } \\ \text { MWPC } & \text { multiwire proportional chamber } \\ \text { PHOTON } & \text { photon spectrometer such as NaI or Ge detectors } \\ \text { PLASTIC } & \text { Lexan, etc., used like emulsion } \\ \text { PMT } & \text { various photomultiplier tubes } \\ \text { RICH } & \text { ring-imaging Cerenkov detector } \\ \text { SAS } & \text { single-arm spectrometer } \\ \text { SCINT } & \text { scintillator } \\ \text { SPEC } & \text { spectrometer system } \\ \text { STRC } & \text { streamer chamber } \\ \text { TRACK } & \text { tracking system } \\ \text { WIRE } & \text { wire chamber }\end{array}$

We use the following acronyms for specific detectors (both in this Report and in the SLAC/SPIRES database):

\begin{tabular}{|c|c|}
\hline ALEPH & CERN-LEP detector \\
\hline ALPHA & JINR magnetic spectrometer system \\
\hline AMY & KEK-TRISTAN high-resolution lepton detector \\
\hline ARGUS & DESY-DORIS-II detector \\
\hline ATLAS & CERN-LHC proposed detector \\
\hline BABAR & SLAC-PEP2 proposed detector \\
\hline BELLE & KEK-B-FACTORY proposed detector \\
\hline BES & $\mathrm{BEPC}$ detector \\
\hline BRAHMS & BNL-RHIC proposed Broad Range Hadron Magnetic Spectrometer \\
\hline $\mathrm{CCM}$ & FNAL-TEV Chicago Cyclotron Magnet spectrometer \\
\hline CDF & Collider Detector at Fermilab \\
\hline CHARM-II & CERN-SPS upgrade of CHARM neutrino detector \\
\hline CLAS & CEBAF Large Acceptance Spectrometer \\
\hline CLEO & CESR spectrometer system \\
\hline CMD-2 & Novosibirsk upgrade of CMD detector \\
\hline CMS & CERN-LHC proposed detector \\
\hline CRYSTAL-BALL & SLAC-PEP, DESY-DORIS, BNL Crystal Ball large-solid-angle neutral detector \\
\hline CRYSTAL-BARREL & CERN-LEAR large-solid-angle detector \\
\hline DELPHI & CERN-LEP detector \\
\hline Do & FNAL-COLLIDER detector \\
\hline EMC & CERN-SPS European Muon Collaboration detector \\
\hline EXCHARM & Serpukhov detector, upgrade of BIS-2M \\
\hline GAMS-4000 & CERN-SPS $64 \times 64$ cell Pb-glass array \\
\hline GAMS-4PI & Serpukhov gamma spectrometer \\
\hline GGNT & Baksan Gallium-Germanium Neutrino Telescope \\
\hline HELIOS & CERN-SPS detector \\
\hline HLBC-DIANA & ITEP heavy liquid bubble chamber \\
\hline HLBC-SKAT & Serpukhov heavy liquid bubble chamber \\
\hline HYPERON-II & Serpukhov single arm magnetic spectrometer \\
\hline H1 & DESY-HERA detector \\
\hline ICARUS & Gran Sasso bubble chamber detector \\
\hline ISTRA-M & Serpukhov detector \\
\hline
\end{tabular}




\section{DETECTORS}

\begin{tabular}{|c|c|}
\hline JETSET & CERN-LEAR compact general purpose detector \\
\hline KEDR & NOVO-VEPP-2M universal magnetic detector \\
\hline KLOE & Frascati DA $\Phi N E$ detector \\
\hline LAB-E & FNAL-TEV target-calorimeter muon-spectrometer detector for neutrino physics \\
\hline LAMBDAMETER & ITEP detector \\
\hline LSND & LAMPF Liquid Scintillator Neutrino Detector \\
\hline L3 & CERN-LEP detector \\
\hline MACRO & Gran Sasso detector \\
\hline MEGA & LAMPF array of electron and photon spectrometers \\
\hline MIS & Serpukhov multiparticle spectrometer \\
\hline MIS-2 & Serpukhov upgrade of MIS \\
\hline MPS & BNL MultiParticle Spectrometer \\
\hline MPS-II & BNL upgrade of MPS \\
\hline MTS & ITEP detector \\
\hline NEPTUN & Serpukhov-UNK jet target detector \\
\hline NMS & LAMPF Neutral Meson Spectrometer \\
\hline OMEGA & CERN, CERN-SPS spectrometer system \\
\hline OMEGAPRIME & CERN-SPS spectrometer system \\
\hline OPAL & CERN-LEP detector \\
\hline PHENIX & BNL-RHIC photon, electron, and hadron detector, under construction \\
\hline PHOBOS & BNL-RHIC two-arm multiparticle spectrometer, under construction \\
\hline PINOT & Saclay high resolution pi0 and eta detector \\
\hline QUARTZ & Serpukhov crystal-diffraction spectrometer \\
\hline SINDRUM & PSI large-solid-angle magnetic detector \\
\hline SINDRUM-II & PSI upgraded large-angle solenoid detector \\
\hline SLD & SLAC-SLC detector \\
\hline SND & Novosibirsk Spherical Neutral Detector \\
\hline SPES-0 & Saclay modular lead-glass Čerenkov detector \\
\hline SPES-II & CERN, CERN-LEAR high-resolution spectrometer \\
\hline SPES-III & Saclay high-resolution spectrometer \\
\hline SPES-IV & Saclay high-resolution spectrometer \\
\hline SPES4-PI & Saclay high-resolution spectrometer \\
\hline SPHINX & Serpukhov detector, also known as SFINKS \\
\hline STAR & BNL-RHIC solenoidal detector, under construction \\
\hline TOKIWA & KEK-PS spectrometer \\
\hline TOPAZ & KEK-TRISTAN solenoidal spectrometer with TPC \\
\hline TPS & FNAL Tagged Photon Spectrometer \\
\hline VENUS & KEK-TRISTAN Versatile Economical and Novel Universal Spectrometer \\
\hline VES & Serpukhov magnetic VErtex Spectrometer \\
\hline ZEUS & DESY-HERA detector \\
\hline
\end{tabular}




\section{SUMMARIES OF BEIJING AND BROOKHAVEN EXPERIMENTS}

\section{BEPC Experiments}

\section{BEPC-BES}

(Began data-taking 1991, In progress)

\section{MEASUREMENT OF THE $\tau$ LEPTON MASS WITH} THE BEIJING SPECTROMETER (BES)

BES COLLABORATION

BEIJING, IHEP - J Z Bai, S M Chen, $S \mathrm{~J}$ Chen, $\mathrm{X} Q$ Cheng,

$\mathrm{Z} D$ Cheng, $\mathrm{H}$ C Cui, $X \mathrm{Z}$ Cui, $\mathrm{H} \mathrm{L}$ Ding, $Z \mathrm{Z}$ Du, $\mathrm{C}$ Fang

M L Gao, S Q Gao, W X Gao, Y N Gao, J H Gu, S D Gu,

W X Gu, Y N Guo, Y Y Guo, Y Han, J He, G Y Hu, H B Hu,

$T$ Hu, D Q Huang, $Y Z$ Huang, $C H$ Jiang, $Z J$ Jiang, $Y$ F Lai,

P F Lang, F Li, J Li (Spokesperson), P Q Li, Q M Li, R B Li,

W D Li, W Li, W G Li, Y S Li, S Z Lin, H M Liu, Q Liu,

R G Liu, Y Liu, J G Lu, D H Ma, E C Ma, J M Ma, H S Mao,

Z P Mao, X C Meng, H L Ni, L J Pan, N D Qi, Y K Que,

$G$ Rong, Y Y Shao, D L Shen, H Y Sheng, H Z Shi, X F Song,

$H$ S Sun, $G$ L Tong, $L Z$ Wang, $M$ Wang, $P$ L Wang, $P$ Wang,

T J Wang, $Y Y$ Wang, X D Wu, D M Xi, X M Xia, P P Xie,

$X X X i e, R S X u, Z Q X u, S T X u e, J$ Yan, $W$ G Yan,

C Y Yang, C M Yang, H B Yao, $M H$ Ye, S $Z$ Ye, $Z$ Q Yu,

B Y Zhang, C C Zhang, D H Zhang, H Y Zhang, H L Zhang,

J W Zhang, L S Zhang, S Q Zhang, Y Zhang, D X Zhao,

M. Zhao, P D Zhao, W R Zhao, J P Zheng, L S Zheng,

Z P Zheng, G P Zhou, H S Zhou, L Zhou, L Zhou, X F Zhou,

Y H Zhou, Q M Zhu, Y S Zhu, Y C Zhu

BOSTON U - J A Coller, A S Johnson, J Shank, J S Whitaker

CA.L TECH - M Hatanaka, D Hitlin, L A Jones, M H Kelsey,

J H Panetta, F Porter, E N Prabhakar, X Shi

COLORADO STATE U - J Chen, Q P Jia, $\underline{\mathrm{W}}$ Toki

(Spokesperson), R J Wilson

HAWAII U - A Breakstone, F Harris, S Olsen, D Paluselli

MTT, LNS - O Bardon, R Cowan, M Fero, J Quigley, E Torrence, R K Yamamoto

SLAC - R A Becker-Szcndy, W M Dunwoodie, H Marsiske, E Soderstrom, J Synodinos, W J Wisniewski

TEXAS U, DALLAS - I Blum, J S Campbell, P Gratton,

J M Izen, X Lou, B Lowery, J Standifird

UC, IRVINE - A J Lankford, M Mandelkern, M Schernal,

B Schmid, J Schultz, A Smith, D P Stoker, G Zioulas

WASHINGTON U, SEATTLE - T Burnett, $\mathrm{K}$ Young

Accelerator BEPC Detector BES

Reactions

$$
e^{+} e^{-} \rightarrow \tau^{+} \tau^{-} \quad 3.6 \mathrm{GeV}\left(\mathrm{E}_{\mathrm{cm}}\right)
$$

Particles studied $\tau$

Brief description Uses non-collinear 2-prong $e \mu$ events with both $e$ and $\mu$ identified. Measures the threshold behavior of the cross-section by scanning a very narrow range of energies, $3.544<E_{\mathrm{cm}}<3.569 \mathrm{GeV}$. The BES detector consists of a Muon Counter, TOF Counters, a Barrel Shower Counter and the main Drift Chamber. A major upgrade of the detector will be completed in the Fall 1996, and data-taking continued.

Journal papers NIM $\Lambda 308$ (1991) 616, PRL 69 (1992) 3021, PL

B355 (1995) 374 [erratum: PL B363 (1995) 267), PRL 74 (1995) 4599, PR D52 (1995) 3781, PR D53 (1996) 20, and PR D54 (1996) 1221.

E-mail contact lij@bepc2.ihep.ac.cn, toki@lamar.colostate.edu WWW Home-page

http://www-bes.slac.stanford.edu/beswww/bes.html

\section{BEPC Future Plans}

The upgrade of the BES spectrometer, which began in 1993, is nearing its completion, which promises new important physics results in the next few years at BEPC. The Lab is also actively involved in the design of the Beijing Tau Charm Factory (BTCF) which got positive reviews at home and abroad. B'TCF was the main topic of a recent workshop in Beijing.

\section{Selected BNL Experiments}

\section{BNL-787}

(Proposed Sep 1983, Approved Oct 1983, Began data-taking Jun 1988, In progress)

\section{A STUDY OF THE DECAX $K^{+} \rightarrow \pi^{+} \nu \bar{\nu}$}

BROOKHAVEN - S Adler, M S Atiya, I H Chiang, M Diwan, J S Frank, E Garber, I S Haggerty, S H Kettell, T F Kycia,

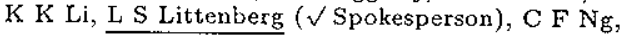

A Sambamurti, $A$ J Stevens, $R C$ Strand, $C$ Witzig, $Y$ Zhao

TOKYO U, INS - M Kazumori, T Komatsubara, M Kuriki,

T Morimoto, N Muramatsu, H Okuno, K Omata, A Otomo, $S$ Sugimoto, K Tauchi, K Ukai

KEK - M Aoki, T Inagaki, S Kabe, M Kobayashi, Y Kuno, T Sato, T Shinkawa, $\mathrm{Y}$ Yoshimura

OSAKA U .- Y Kishi, T Nakano, T Sasaki

PRINCETON U - D Akerib, M Ardebili, M Convery, M M Ito,

D R Marlow, R McPherson, P D Meyers, W Sands, M A Selen, F C Shoemaker, A J S Smith ( $\checkmark$ Spokesperson), J R Stone TRIUMF - P Bergbusch, E W Blackmore, D A Bryman

( $\checkmark$ Spokesperson), M Burke, J V Cresswell, A Daviel, S Daviel, $P$ Kitching, A Konaka, M LeNoble, J A Macdonaid, J Mitdenberger, T Numao, P Padley, I M Poutissou, R Poutissou,

G Redlinger, J Roy, M Rozon, R Soluk, A S Turcot

Accelerator BNL Detector Spectrometer

\section{Reactions}

$$
\begin{aligned}
& K^{+} \rightarrow \pi^{+} \nu \bar{\nu} \\
& K^{+} \rightarrow \pi^{+} \mu^{+} \mu^{-} \\
& K^{+} \rightarrow \mu^{+} \mu^{+} \mu^{-} \nu \\
& K^{+} \rightarrow \mu^{+} \nu \gamma \\
& K^{+} \rightarrow \pi^{+} \text {higgs } \\
& K^{+} \rightarrow \pi^{+} \gamma \gamma \\
& K^{+} \rightarrow \pi^{+} \mathrm{X} \\
& K^{+} \rightarrow \pi^{+} \pi^{0} \gamma \\
& \pi^{0} \rightarrow \nu \bar{\nu} \\
& \pi^{0} \rightarrow \gamma \mathrm{X} \\
& \pi^{0} \rightarrow \gamma \nu \bar{\nu}
\end{aligned}
$$

Particles studied $K^{+}$, higgs, nuino, $\pi^{0}$

Brief description A sensitivity down to a level of $1 \times 10^{-10}$. or better, is expected for $K^{+} \rightarrow \pi^{+} \nu \bar{\nu}$. A measurement at this level determines $\left|V_{t d}\right|$, given that $m_{t}$ is known. An observation significantly above this level would indicate a fourth gencration of quarks and leptons, the presence of nuinos, or other new phenomena. A simultancous measurement of $\mathrm{K}^{+} \rightarrow \pi^{+} X$ to a sensitivity of about $2 \times 10^{-11}$ is also expected. This probes the existence of axions, familons, hyperphotons, or other new particles. Other processes probe the existence of higgs, majorons, massive neutrinos, and other hypothetical particles. The first run was completed in Junc 91 . Approved for 3000 additional hours with an upgraded beam and detector. The new beam $\left(730 \mathrm{MeV} / \mathrm{c} \mathrm{K}^{+}\right.$, stopped in spectrometer) was commissioned in 1992, the modified detector in 1994, and data was already collected with the new setup in 1995/96. Data taking is scheduled to resume in early 1997.

Journal papers NIM A279 (1989) 180, PRL 63 (1989) 2177, PRL 64 (1990) 21, PRL 65 (1990) 1188, NP (PROC SUPPL) 13 (1990) 568, PRL 66 (1991) 2189, NMM A321 (1992) 129, PRL 69 (1992) 733, PRL 70 (1993) 2521 [erratum: PRL 71 (1993) 305], PR D48 (1993) 1, PR D48 (1993) 1225, and PRY 76 (1996) 2421.

Related experiments N/A

E-mail contact littenbe@bnl.gov, smith@puphep.princeton.edu, doug@sitka.triumf.ca

WWW Home-page http://www.phy.bnl.gov/e787/e787.html 


\section{SUMMARIES OF BROOKHAVEN EXPERIMENTS}

\section{BNL-821}

(Proposed Scp 1985, Sep 1986, Approved Nov 1986, In preparation)

A NEW PRECISION MEASUREMENT OF THE $g_{\mu}-2$ VALUE AT THE LEVEL OF 0.35 PPM

MUON (G-2) COLLABORATION

BOSTON U - D H Brown, R M Carey, W Earle, E Efstathiadis, E S Hazen, B J Hughes, F Krienen, J P Miller, O Rind, B L Roberts ( $\sqrt{ }$ Spokesperson), L R Sulak, W A Worstell

BROOKFAVEN - $J$ T Benante, $\mathrm{H} N$ Brown, $G$ Bunce,

J R Cullen, G $T$ Danby, C R Gardner, J M Geller, H Hseuh,

J W Jackson, L- Jia, R Larsen, Y Y Lee, R E Meier, W Meng,

W M Morse ( $\sqrt{ }$ Spokesperson), C Pai, I Polk, R Prigl,

S Rankowitz, J Sandberg, Y Semertzidis, R Shutt, L Snydstrup,

A Soukas, A Stillman, $T$ Tallerico, P Thompson, F Toldo,

$\mathrm{K}$ Woodle

CORNELL U - T Kinoshita, Y Orlov

FAIRFIELD U - D Winn

HEIDELBERG U, PHYS INST - J Gerhaeuser, A P Grossmann,

$K$ Jungmann, $P$ von Walter, G zu Putlitz

HEIDELBERG, MAX PLANCK INST -- U Haeberlen

ILLINOIS U, URBANA - P T Debevec, W Deninger,

D W Hertzog, S A Sedykh, D C Urner

LBL \& BROOKHAVEN - M A Green

MINNESOTA U - P B Cushman, S O Giron, J M Kindem,

D E Maxam, D E Miller, C Timmermans, D Zimmerman

NOVOSIBIRSK, IYF - L M Barkov, D N Grigorev, B I Khazin,

E A Kuraev, Y M Shatunov, E Solodov

TOKYO U -. K Nagamine

SCIENCE U, TOKYO - Y Mizumachi

KEK - K Endo, H Hirabayashi, S Ichii, S Kurokawa, T Sato, A Yamamoto

WAKO, RIKEN - K Ishida

YALE U - S K Dhawan, F J M Farley, M Grosse-Perdekamp,

V W Hughes ( $\checkmark$ Spokesperson), D M Kawall, S I Redin

Accelerator BNL Detector Other

Reactions Polarized beam

$$
\text { muon } \rightarrow e^{ \pm}{ }_{\nu} \bar{\nu} \quad 3.09 \mathrm{GeV} / \mathrm{c}
$$

Particles studied muon

Brief description Measures the anomalous gyromagnetic ratio of the muon and provides a direct test of the Standard Model. Uses a 7-m-radius superferric muon storage ring with a $1.45-$ tesla vertical field. Approved for 2100 hours. The first data collection expected in FY 1997.

E-mail contact roberts@bu.edu, morse1@bnl.gov, vernon.hughes@yale.edu

WWW Home-page http://www phy.bnl.gov/g2muon/home.html

\section{BNL-840}

(Proposed Jul 1987, Approved Oct 1987, Began data-taking Jul 1989, Completed data-taking Dec 1991)

SEARCH FOR THE COHERENT PRODUCTION OF LIGHT SCALAR AND PSEUDOSCALAR PARTICLES

ROCHESTER U - R Cameron, G Cantatore, A C Melissinos ( $\sqrt{ }$ Spokesperson), J T Rogers, G Ruoso, Y K Semertzidis BROOKHAVEN - II Halama, D Lazarus, A G Prodell FERMYLAB - F A Nezrick

CERN \& TRIESTE U - P Micossi, C Rizzo, E Zavattini

Accelerator NONE Detector Other

Particles studied axion

Brief description The detector used two CBA superconducting dipoles. Searched for light scalar or pseudoscalar particles that couple to the electromagnetic field. Looked for optical rotation of a polarized laser beam traversing in vacuum the $3.5 \mathrm{~T}$ magnetic field. The sensitivity of $10^{-10} \mathrm{rad}$ corresponds to a limit on the coupling $g_{a \gamma \gamma}$ of $4 \times 10^{-7} \mathrm{GeV}^{-1}$. Did not reach Delbruck scattering (real photons from virtual photons) below the $e^{+} e^{-}$threshold.

Journal papers PRL 64 (1990) 2988, JOSA B8 (1991) 520, PL A157 (1991) 125, PRL 69 (1992) 2333, ZPHY C56 (1992) 505 and PR D47 (1993) 3707.

Related experiments BNL-805

E-mail contact meliss@urhep.pas.rochester.edu

\section{BNL -852}

(Proposed Jan 1989, Approved Mar 1989, Began data-taking Jun 1993, In progress)

\section{SEARCH FOR MESONS WITH UNUSUAL QUANTUM NUMBERS}

BROOKHAVEN - S U Chung ( $\sqrt{ }$ Spokesperson), R W Hackenburg, K Olchanski, D P Weygand, H J Willutzki INDIANA U - B B Brabson, R R Crittenden, A R Dzierba, P C Figliozzi, J L Gunter, B Kern, R Lindenbusch, M McKerley, D R Rust, E B Scott, P T Smith, T Sulanke, S Teige MASSACHUSETTS U, DARTMOUTH - Z Bar-Yam, I Cummings, I Dowd, P Eugenio, W Kern, E King MOSCOW STATE U - E V Anoshina, L I Belzer, V A Bodyagin, A Demianov, A M Gribushin, O L Kodolova, V L Korotkikh, M A Kostin, N Kruglov, A I Ostrovidov, A S Proskuryakov, L I Sarycheva, N B Sinev, I N Vardanyan, A A Yershov NOTRE DAME U - T Adams, J M Bishop, N M Cason

( $\sqrt{ }$ Spokesperson), E I Ivanov, J M LoSecco, J J Manak, A Sanjari, W D Shephard, D L Stienike, S A Taegar,

D R Thompson

NORTHWESTERN U - D S Brown, T Pcdlar, K K Seth, J Wise, D Zhao

SERPUKHOV - S Denisov, A Dushkin, V Kochetkov, V Lipaev,

I Shein, A Soldatov

RENSSELAER POLY - G Adams, J Kuhn, 3 Napolitano, M Nozar, J Smith, D B White, M Witkowski, A M Wright

Accelerator BNL Detector MPS

$$
\begin{aligned}
& \text { Reactions } \\
& \pi^{-} p \rightarrow n \eta \pi^{0} \\
& \pi^{-} p \rightarrow n \eta \eta \\
& \pi^{-} p \rightarrow p \eta \pi^{-} \\
& \pi^{-} p \rightarrow p \eta^{\prime} \pi^{-} \\
& \pi^{-} p \rightarrow p \eta \pi^{-} \pi^{+} \pi^{-} \\
& \pi^{-} p \rightarrow p \pi^{-} \pi^{+} \pi^{-} \\
& \pi^{-} p \rightarrow n \eta \pi^{0} \pi^{0} \\
& \pi^{-} p \rightarrow n \eta \pi^{+} \pi^{-} \\
& \pi^{-} p \rightarrow n \eta \eta^{\prime} \\
& \pi^{-} p \rightarrow n K^{0} \bar{K}^{0} \pi^{0} \\
& \pi^{-} p \rightarrow n K^{0} \ddot{K}^{0} \pi^{0} \pi^{0} \\
& \pi^{-} p \rightarrow n K^{0} \bar{K}^{0} \pi^{+} \pi^{-}
\end{aligned}
$$

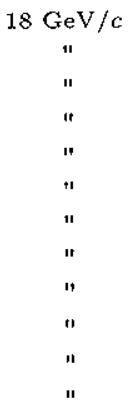

Particles studied exotic-meson, glueball, hybrid

Brief description Looks in particular for further evidence of an " $M(1405)$ ", observed to decay into $\eta \pi^{\circ}$ in GAMS-spectrometer experiments at Serpukhov and CERN. Studies decay modes of mesons, with multiphotons and 0,1 , or 2 charged particles. The detector is built around MPS. An $\mathrm{H}_{2}$ target is surrounded by a CsI veto. Other parts of the apparatus are a charged particle detector, a Cerenkov counter, and a 3000-element lead glass calorimeter. Approved for a total of 5000 hours. 'Taking data (July 96).

Journal papers NIM A332 (1993) 419, A342 (1994) 398, NIM A357 (1995) 95, and NIM A368 (1996) 617 .

Related experiments SERPUKHOV-163, CERN-NA-012-2

E-mail contact suchung@bnlarm.bnl.gov, neal.m.cason.1@nd.edu WWW Home-page

http://lemond.phy.bnl.gov/ $\sim$ e852/home_e852.html 


\section{SUMMARIES OF BROOKHAVEN EXPERIMENTS}

\section{BNL-865}

(Proposed May 1990, Approved Jun 1990, Began data-taking 1995 In progress)

\section{IMPROVED SEARCH FOR $K^{+} \rightarrow \pi^{+} \mu^{+} e^{-}$}

E865 COLLABORATION

BASEL U - G Backenstoss, W Menzel, H Weyer

BROOKHAVEN - D Lazarus, L Leipuner, H Ma, P Rehak

MOSCOW, INR - G S Atoyan, V V Isakov, O Karavichev,

A A Poblaguev, V Postoev, I N Semeniouk

NEW MEXICO U - B Bassalleck, S W Eilerts, J Lowe, D Wolfe

PSI, VILLIGEN - J Egger, W D Herold, H Kaspar, J Missimer

PITTSBURGH U - R Appel, N E Cheung, C A Felder,

H M Gach, D E Kraus, I G Ober, P A Pomianowski, A Sher,

$\mathrm{J}$ A Thompson

TBILISI STATE U - Y S Bagaturia, D Mazavia, G V Melitauri, T M Sakhelashvili

YALE U - D R Bergman, H D Do, J A Lozano, W A Majid, M E Zeller ( $\sqrt{ }$ Spokesperson)

ZURICH U - S Pislak, P Robmann, P Truoel

Accelerator BNL Detector Spectrometer, Calorimeter

Reactions

$$
\begin{array}{lc}
K^{+} \rightarrow \pi^{+} \mu^{+} e^{-} & 6.0 \mathrm{GeV} / c\left(P_{1 a b}\right) \\
K^{+} \rightarrow \pi^{+} e^{+} e^{-} & " \\
K^{+} \rightarrow \pi^{+} \mu^{+} \mu^{-} & " \\
K^{+} \rightarrow \pi^{+} \pi^{0} e^{+} e^{-} & " \\
K^{+} \rightarrow \pi^{+} \pi^{-} e^{+} \nu & " \\
\pi^{0} \rightarrow e^{+} e^{-} & -
\end{array}
$$

Particles studied $K^{+}, \pi^{0}$

Brief description Continuation of BNL -777 experiment, with a factor of approximately 70 improved sensitivity. The main purpose is to observe, or place a limit of a few times $10^{-12}$ on the branching ratio of the muon number violating decay $K^{+} \rightarrow \pi^{+} \mu^{+} e^{-}$. Uses a magnetic spectrometer system with very high rate, and good particle identification. Aims at achieving significant improvements on measurements of rates and kinematic distributions of other rare decays (B.R. $<10^{-4}$ ) Taking data (July 96).

Related experiments BNL-777

E-mail contact michael.zeller@yale.edu

WWW Home-page

http://130.132.48.201/www_. info/e865/e865.html

\section{BNL-871}

(Proposed Sep 1990, Approved Nov 1990, Began data-taking Feb 1995, In progress)

\section{A NEW SEARCH FOR VERY RARE $K_{L}$ DECAYS}

UC, IRVINE - V Abadjev, M G Bachman, D F Connor, $P$ DeCecco, R M Djilibaev, $N$ Kanematsu, R K Lee, W R Molzon ( $\checkmark$ Spokesperson)

STANFORD U - C G Arroyo, K W Hartman, M J Hebert,

$G$ M Irwin, D Ouimette, M C Pommot Maia, S G Wojcicki ( $\checkmark$ Spokesperson)

TEXAS U - D Ambrose, S C Graessle, K Lang, A Milder,

J L Ritchie ( $\checkmark$ Spokesperson), V Vassikakopoulos

WILLIAM AND MARY COLL - M Eckhause, A D Hancock,

$C$ Hoff, J R Kane ( $\checkmark$ Spokesperson), Y Kuang, $R$ D Martin,

R E Welsh, E J Wolin

RICHMOND U - P D Rubin

Accelerator BNL Detector Spectrometer

Reactions

$K_{L} \rightarrow \mu^{-} e^{+}$
$K_{L} \rightarrow \mu^{+} e^{-}$
$K_{L} \rightarrow \mu^{+}{ }^{-}$
$K_{L} \rightarrow e^{+} e^{-}$

$K_{L} \rightarrow e^{+} e^{-}$

Particles studied $K_{L}$
Brief description A search for the decays $K_{L} \rightarrow \mu e$ and $K_{L} \rightarrow$ ee with a sensitivity of $10^{-12}$, and a measurement of the branching fraction for $K_{L} \rightarrow \mu \mu$. BNL-871 improves on the sensitivity of BNL-791 by more than a factor of ten. The detector consists of two dipoles, straw and wire drift chambers, scintillation and gas Cerenkov counters, a lead glass shower counter, and a muon rangefinder. A novel feature of the experiment is a shielded tungsten beam stop inside the spectrometer. Test data were taken in 1991/92, engineering runs were done in 1993/94, and the actual data taking began with a 5 -month run in 1995. Approved for 7,000 hours. In progress (1996).

Related experiments BNL-791

E-mail contact wmolzon@uci.edu, sgweg@slac.stanford.edu, ritchie@utaphy.ph.utexas.edu, kane@wmheg.physics.wm.edu WWW Home-page http://www.ps.uci.edu/ e871/e871intro.html

\section{BNL-881}

(Proposed Jul 1991, Approved Aug 1991, In progress)

UTILIZING $\phi \phi$ SPECTROSCOPY TO SEARCH FOR EXOTIC GLUEBALLS, EXOTIC HYBRID, OR EXOTIC MULTIQUARK STATES

BROOKHAVEN \& CITY COLL, NY - S J Lindenbaum (Spokesperson)

BROOKHAVEN - R W Hackenburg, R S Longacre

CITY COLL, NY - C S Chan, E Efstathiadis, M A Kramer,

K Zhao, Y Zhu

CONNECTICUT U - M Gai

FERMILAB - K Vaziri

RENSSELAER POLY - G Adams, C Landberg, A M Perry,

B Wojtsekhowski

Accelerator BNL Detector MPS-II

\section{Reactions}

$\pi^{-} p \rightarrow \phi \phi n$

$\pi^{-} p \rightarrow \phi K^{+} K^{-} n$

$\pi^{-} p \rightarrow K^{+} K^{-} K^{+} K^{-} n$

$K^{-} p \rightarrow \phi \phi \Lambda$

$K^{-} p \rightarrow \phi \phi \Sigma$

$K^{-} p \rightarrow \phi K^{+} K^{-} \Lambda$

$K^{-} p \rightarrow \phi K^{+} K^{-} \Sigma$

$K^{-} p \rightarrow K^{+} K^{-} K^{+} K^{-} \Lambda$

$K^{-} p \rightarrow K^{+} K^{-} K^{+} K^{-} \Sigma$

$\bar{p} p \rightarrow \phi \phi \pi^{0}$

$\bar{p} p \rightarrow \phi K^{+} K^{-} \pi^{0}$

$\bar{p} p \rightarrow K^{+} K^{-} K^{+} K^{-} \pi^{0}$

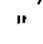

"

$"$

$n$

"

$"$

$"$

$"$

11

Particles studied glueball, $f_{2}(2010), f_{2}(2300), f_{2}(2340)$

Brief description A search for exotic glueballs and exotic hybrids. Uses the MPS facility and the MESB beam at $8 \mathrm{GeV} / c$, tuned to contain $\pi^{-}, K^{-}$, and $\ddot{p}$. Lowering the momentum to 8 $\mathrm{GeV} / c$ increases the rate of exchanges required to make exotics. Currently pausing (May 96).

E-mail contact lindenbaum@bnldag.bnl.gov

WWW Home-page

http://www.phy.bnl.gov/ e881/welcome_ags.html

\section{BNL-909}

(Proposed Oct 1994, Approved Nov 1994, Began data-taking Apr 1995, Completed data-taking May 1995)

ETA PRODUCTION AT THRESHOLD IN THE REACTIONS $\pi^{-} p \rightarrow \eta n$ AND $K^{-} p \rightarrow \Lambda \eta$

ABILENE CHRISTIAN U - L D Isenhower, A A Rose, M Sadler BOŠKOVIĆ INST, ZAGREB - A Marušić, I S̆laus BROOKHAVEN - $\mathrm{R}$ Chrien, R Sutter

DUBNA - A A Efendiev 


\section{SUMMARIES OF BROOKHAVEN EXPERIMENTS}

GEORGE WASHINGTON U - C Bennhold, W J Briscoe

( $\checkmark$ Spokesperson), T W Morrison, Z Papandreou, S A Philips, R Pratt, A Shafi

HOUSTON $U-R$ Sawafta

RENSSELAER POLY - D B White

ST PETERSBURG, INP - V V Abaev, V Bekrenev

ST PETERSBURG, INP - N Kozlenko

UCLA - M Clajus, S C McDonald, B M K Nefkens, W B Tippens

( $\checkmark$ Spokesperson)

Accelerator BNL Detector Spectrometer

Reactions

$$
\begin{array}{cc}
\pi^{-} p \rightarrow \eta n & 680-760 \mathrm{MeV} / c\left(P_{\text {lab }}\right) \\
K^{-} p \rightarrow \eta \Lambda & "
\end{array}
$$

Particles studied $N\left(1535 S_{11}\right), \Lambda\left(1670 S_{01}\right)$

Brief description Measures the total cross-section, as well as angular distribution for $\eta$ production from threshold ( $\mathrm{P}=685$ $\mathrm{MeV} / c$ for pion, $\mathrm{P}=723 \mathrm{MeV} / c$ for kaon) up to $760 \mathrm{MeV} / c$. The $\eta$ particles are detected via the $2 \gamma$ decay mode using the improved $\eta$ spectrometer. Data analysis in progress at GWU (July 96).

Related experiments BNL-890, BNL-913, BNL-914

E-mail contact briscoe@gwis2.circ.gwu.edu, btippens@uclapp.physics.ucla.edu

WWW Home-page

http://ucla5.phy.bnl.gov/htdocs/e909/e909.html

\section{BNL-913-914}

(Proposed Mar 1995, Approved Sep 1995, In preparation)

\section{BARYON AND NEUTRAL HYPERON SPEC-} TROSCOPY WITH THE CRYSTAL BALL

\section{CRYSTAL-BALL COLLABORATION}

ABILENE CHRISTIAN U - B Draper, J Huddleston,

$L D$ Isenhower, $Z$ Mulkey, $M$ E Sadler ( $\checkmark$ Spokesperson)

ARGONNE - H Spinka ( $\sqrt{ }$ Spokesperson)

ARIZONA STATE U - J R Comfort, K Craig

BOŠKOVIĆ INST, ZAGREB - M Batinić, I Š̉laus, I Supek,

A Švarc

BROOKHAVEN - T Kycia ( $\checkmark$ Spokesperson)

COLORADO U - R J Peterson

GEORGE WASHINGTON U - C Bennhold, W J Briscoe

KARLSRUHE U - H M Staudenmaier

KENT STA'TE U - D M Manley

ST PETERSBURG, INP V V Abaev, V S Bekrenev,

N G Kozlenko, S P Kruglov ( $\checkmark$ Spokesperson), I V Lopatin,

A B Starostin

REGINA U - G J Lolos, Z Papandreou

UCLA - M Clajus, A Marušić, S C McDonald, B M K Nefkens

( $\checkmark$ Spokesperson), W B Tippens ( $\checkmark$ Spokesperson)

VALPARAISO U, INDIANA - D Groznick, D D Koetke,

R W Manweiler, S Stanislaus

Accelerator BNL Detector CRYSTAL-BALL

\section{Reactions}

$$
\begin{aligned}
& \pi^{-} p \rightarrow n \gamma \\
& \pi^{-} p \rightarrow \pi^{0} n \\
& \pi^{-} p \rightarrow \pi^{0} \pi^{0} n \\
& \pi^{-} p \rightarrow \eta n \\
& \pi^{-} p \rightarrow \pi^{0} \eta n \\
& \pi^{-} p \rightarrow \omega n \\
& K^{-} p \rightarrow \Lambda \gamma \\
& K^{-} p \rightarrow \Lambda \pi^{0} \\
& K^{-} p \rightarrow \Lambda 2 \pi^{0} \\
& K^{-} p \rightarrow \Lambda \eta \\
& K^{-} p \rightarrow \Sigma^{0} \gamma \\
& K^{-} p \rightarrow \Sigma^{0} \pi^{0} \\
& K^{-} p \rightarrow \Sigma^{0} 2 \pi^{0} \\
& K^{-} p \rightarrow \Sigma^{0} \eta
\end{aligned}
$$

$400-1800 \mathrm{MeV} / \mathrm{c}$

$$
"
$$$$
\text { it }
$$

$685-1800 \mathrm{MeV} / \mathrm{c}$ $1000-1800 \mathrm{MeV} / \mathrm{c}$ $1100-1800 \mathrm{MeV} / \mathrm{c}$ $600-1800 \mathrm{McV} / \mathrm{c}$$$
\text { " }
$$

$720-1800 \mathrm{MeV} / \mathrm{c}$ $600-1800 \mathrm{MeV} / \mathrm{c}$

$$
"
$$$$
\text { " }
$$

$890-1800 \mathrm{MeV} / \mathrm{c}$

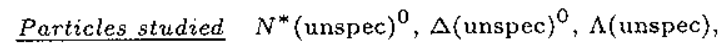
$\Sigma{\text { (unspec })^{0}}^{0}$

Brief description Measures absolute differential cross-sections. Uses the Crystal Ball multiphoton spectrometer with large acceptance and high energy resolution. Various reaction channels are identified by reconstructing the invariant mass of the produced $\gamma$ rays. The experiment has two distinct parts. The goal of BNL-913 is a better determination of the masses, widths, and decay modes of the excited states of the nucleon. The spokespersons are M.E. Sadler, H. Spinka and W.B. Tippens. BNL-914 studies the $\Lambda^{*}$ and $\Sigma^{*}$ resonances via their neutral decays. The incident beam momentum is varied in steps of $25-50 \mathrm{MeV} / c$ from $600 \mathrm{MeV} / c$ in the C-line to the maximum momentum of $1.8 \mathrm{GeV} / c$ in the $D$-line. The spokespersons are T. Kycia, S.P. Kruglov and B.M.K. Nefkens. Approved for 1000 hours. Scheduled to run in FY 1997.

\section{E-mail contact}

sadler@physics.acu.edu,hms@hep.anl.gov, kycia@bnl.gov, kruglov@lnpi.spb.su,btippens@uclapp.physics.ucla.edu, bnefkens@uclapp.physics.ucla.edu

WWW Home-page

http://ucla5.phy.bnl.gov/htdocs/e913-914/e913-914.html

\section{BNL-RHIC-BRAHMS}

(Proposed 1992, Approved Apr 1995, In preparation)

\section{THE BRAHMS EXPERIMENT AT RHIC}

\section{BRAHMS COLLABORATION}

\section{Accelerator BNL-RHIC Detector BRAHMS}

Brief description RHIC provides an opportunity for experiments investigating both baryon poor quark gluon plasma in the midrapidity region and baryon rich plasma in the fragmentation regions of rapidity. The most basic information available for understanding the phenomena that occur in heavy ion collisions comes from the momentum spectra and yields of the various emitted particles as a function of transverse momentum and rapidity. One of the goals of the experiment is to measure the spectra of well identified charged hadrons over a wide range of rapidity and transverse momenta as a function of the centrality of the collision. Uses the BRAHMS (Broad RAnge Hadron Magnetic Spectrometers) detector, consisting of a magnetic forward angle hadron spectrometer and a midrapidity spectrometer. PAC approved in Apr 95. For further details, please contact the Spokesperson, Dr. Flcmming Videbaek [BNL], or get in touch with Dr. Dana Beavis [BNL].

E-mail contact videbaek@bnl.gov, beavis@bnl.gov WWW Home-page

http://rsgi01.rhic.bnl.gov/export1/brahms/WWW/brahms.html

\section{BNL-RHIC-PHENIX}

(Proposed 1989, In preparation)

PHOTON-ELECTRON NEW HEAVY ION EXPERIMENT

\section{PHENIX COLLABORATION}

Accelerator BNL-RHYC Detector PHENIX

Brief description Studies simultaneously various signatures of quark-gluon plasma in heavy ion collisions. Focuses specifically on the measurement of leptons, photons, and identified hadrons, and should be capable of exploiting the highest luminosities envisioned for RHIC. Studies also asymmetries of photons and leptonic decays of weak bosons in polarized proton reactions. The PHENIX detector system is based on an axial field magnet in which the central rapidity interval is covered by two detector arms, each subtending $90^{\circ}$ in azimuth. The aperture is instrumented to detect electrons, photons, and hadrons. Two muon arms, covering polar angles subtending $30^{\circ}$ (forward and backward), have a good acceptance for muon pairs and allow also electron-muon coincidence measurements. An event multiplicity array to cover over five rapidity units is used for 


\section{SUMMARIES OF BROOKHAVEN EXPERIMENTS}

event characterization. Under construction (July 96). The collaboration consists of over 420 scientists, engineers, and graduate students from 45 participating institutions in 10 countries. For further details, please contact the Spokesperson, Prof. Shoji Nagamiya [Columbia U.]

$\underline{E-m a i l ~ c o n t a c t}$ nag@nevis.nevis.columbia.edu WWW Home-page

http://rsgi01.rhic.brl.gov/ $\sim$ phenix/phenix_home.html

\section{BNL-RHIC-PHOBOS}

(Proposed Jan 1992, Approved Nov 1992, In preparation)

PROPOSAL TO STUDY VERY LOW $p_{\perp}$ PHENOMENA AT RHIC

PHOBOS COLLABORATION

Accelerator BNL-RHIC Detector PHOBOS

Brief description The experiment is based on the premise that phase transitions may be signaled by large fluctuations in the overall multiplicity or angular distribution of charged particles. An outer $4 \pi$ detector measures multiplicity and pseudorapidity. A small subset of particles around $y=0$ is then studied in detail by a two-arm multiparticle spectrometer with good momentum resolution and particle identification. The detector is based on silicon technology and allows unbiased triggering at very high rates. In preparation (July 96). For further details, please contact the Spokesperson, Prof. Wit Busza [MIT]

$\underline{E-\text { mail contact }}$ busza@mit.edu

$W W W$ Home-page

http://www.rhic.bnl.gov/phobos/common/WWW/phobos.html

\section{BNL-RHIC-PP2PP}

(Proposed Jan 1994, Approved Mar 1994, In preparation)

TOTAL AND DIFFERENTIAL CROSS-SECTIONS, AND POLARIZATION EFFECTS IN $p p$ ELASTIC SCATTERING AT RHIC

PP2PP COLLABORATION

\section{Accelerator BNL-RHIC Detector ?}

Brief description Studies systematically the pp elastic scattering from $\sqrt{s}=60 \mathrm{GeV}$ to $\sqrt{s}=500 \mathrm{GeV}$, in two kinematical regions. In the Coulomb Nuclear Interference (CNI) region, for $|t|$ between 0.0005 and $0.12(\mathrm{GeV} / \mathrm{c})^{2}$, measures (1) the $s$ dependence of the total and elastic cross-sections, $\sigma_{t o t}$ and $\sigma_{e l},(2)$ the ratio of the real to the imaginary part of the forward elastic scattering amplitude, and (3) the nuclear slope parameter of the $p p$ elastic scattering, $b$. In the intermediate $|t|$ region, $|t| \leq 1.5(\mathrm{GeV} / \mathrm{c})^{2}$, studies the evolution of the dip structure with $s$, and the $s$ and $|t|$ dependence of $b$. The possibility of having polarized proton beams at RHIC would allow measurements of (i) the difference in the total crosssections as a function of initial transverse spin states, (ii) the analyzing power, $A_{N}$, especially in the dip region where a pronounced structure was found at fixed-target experiments, and (iii) the transverse spin correlation parameter, $A_{N N}$. Under construction (July 96). For further details, please contact the Spokesperson, Dr. Wlodek Guryn [BNL]

E-mail contact guryn@bnl.gov

\section{BNL-RHIC-STAR}

(Proposed 1990, Approved Aug 1991, In preparation)

\section{SOLENOIDAL TRACKER AT RHIC (STAR)}

STAR COLLABORATION

Accelerator BNL-RHIC Detector STAR

Brief description STAR is designed to search for signatures of quark-gluon plasma formation and to investigate the behavior of strongly interacting matter at high energy density. The emphasis is on the measurement and correlation of global observables on an event-by-event basis and the use of hard scattering of partons to probe the properties of high density nuclear matter. The event-by-event measurement of global observables is possible because of the very high charged particle densities ( $\mathrm{d} n / \mathrm{d} y$ about 1000) expected at mid-rapidity in nucleus-nucleus collisions at RHIC. To fulfill the physics objectives, the experiment will provide tracking, momentum analysis, and particle identification of most of the charged particles at mid-rapidity. The baseline STAR detector includes a time projection chamber ( $\mathrm{TPC}$ ) in a solenoidal magnetic field of $0.5 \mathrm{~T}$, covering approximately 4 units of the central rapidity. The cylindrical TPC is four meters in diameter. Ionization charge produced along particle trajectories is drifted to the two end plates, where induced signals and arrival times are read out on 150,000 cathode pads. Particle identification will be possible via $\mathrm{d} E / \mathrm{d} x$ in the $1 / \beta^{2}$ region. Upgrades being developed for STAR include an array of TOF counters to extend the PID capabilities to higher momenta, an electromagnetic calorimeter to provide for the measurement of neutral energy, and a silicon vertex detector which uses position sensitive silicon devices with a drift-time measurement technique. $\Lambda$ further upgrade using TPC's in the forward direction will extend the particle tracking coverage to a pseudorapidity of approximately \pm 4 . PAC approved August 91. In preparation (July 96). For further details, please contact the Spokesperson, Prof. John W. Harris [Yale U.]. Project Director is Dr. Jay N. Marx [LBL], and Deputy Spokesperson Dr. Timothy J. Hallman [BNL].

Journal papers NP A566 (1994) 277c.

E-mail contact jwharris@lbl.gov, jnmarx@lbl.gov, tjhallman@lbl.gov

WWW Home-page

http://rsgi01.rhic.bnl.gov/star/starlib/doc/www/star.html

\section{Other BNL Experiments}

Listed here are some other BNL experiments of interest to the particle physics community. Find more details about these projects online, in the SLAC's EXPERIMENTS database (see p. 3), or contact the spokespersons. Check also the annual Brookhaven report 'AGS Experiments' (BNL-34518), or visit the Web sites: http://www.rhichome.bnl.gov/AGS/ (for the AGS experiments), or http://www.rhic.bnl.gov/ (for the RHIC experiments)

BNL-774 (Completed data-taking 1991)

SEARCH FOR $\Sigma$ HYPERNUCLEAR LEVELS IN ${ }^{4} \mathrm{He}$ Spokesperson: E.V. Hungerford [Houston U.]

E-mail contact hunger@uh.edu

BNL-781 (Completed data-taking 1992)

SPIN DEPENDENCE OF THE $A$ - NUCLEUS INTERACTION DETERMINED BY OBSERVATION OF HYPERNUCLEAR $\gamma$ RAYS

Spokespersons: M. May [Brookhaven], M. Deutsch [MIT]

E-mail contact may2@bnl.gov, deutsch@mitlns.mit.edu

BNL-810 (Completed data-taking 1992)

A SEARCH FOR QUARK MATTER (QGP) AND OTHER NEW PHENOMENA UTLLIZING HEAVY ION COLLISIONS AT THE AGS

Spokespersons: E.D. Platner [Brookhaven], S.J. Lindenbaum [Brookhaven and City Coll., N.Y.]

E-mail contact platner@bnldag.bnl.gov, lindenbaum@bnldag.bnl.gov 


\section{SUMMARIES OF BROOKHAVEN EXPERIMENTS}

BNL-813 (Completed data-taking Jun 1995)

SEARCH FOR A STRANGENESS - 2 DIBARYON

Spokespersons: G.B. Franklin [Carnegie Mellon U.], P.D. Barnes [Los Alamos]

E-mail contact franklin@ernest.phys.cmu.edu, pdbarnes@lanl.gov

BNL-814 (Completed data-taking 1992)

STUDY OF EXTREME PERIPHERAL COLLISIONS AND OF THE TRANSITION FROM PERIPHERAL TO CENTRAL COLLISIONS IN REACTIONS INDUCED BY R.ELATIVISTIC HEAVY IONS

Spokesperson: Peter BraunmMunzinger [SUNY, Stony Brook]

E-mail contact pbm@skipper.physics.sunysb.edu

BNL-817 (Taking data)

POLARIZATION TRANSFER IN HYPERON PRODUCTION

Spokespersons: Billy E. Bonner, Jay B. Roberts [Rice U.]

E-mail contact bonner@physics.rice.edu

BNL-836 (Completed data-taking Jul 1994)

SEARCH FOR A STRANGENESS -2 DIBARYON USING $\mathrm{A}^{3} \mathrm{He}$ TARGET

Spokespersons: G.B. Franklin [Carnegie Mellon U.], P.D. Barnes [Los Alamos]

E-mail contact franklin@ernest.phys.cmu.edu, pdbarnes@lanl.gov

BNL-844 (In preparation)

MEASUREMENT OF ANGULAR DISTRIBUTIONS

FOR FRAGMENTS IN THE TARGET RAPIDITY REGION

Spokesperson: James B. Cumming [Brookhaven]

E-mail contact cumming@bnl.gov

BNL-849 (Completed data-taking 1992)

SEARCH FOR MUONIUM TO ANTIMUONIUM CONVERSION

Spokespersons: Dale Richard Harshman, A.P. Mills, Jr. [A T and T Bell Labs, Murray Hill

BNL-850 (Taking data)

EVA, A SOLENOIDAL DETECTOR FOR LARGE AN-

GLE EXCLUSIVE REACTIONS: PHASE I - DETERMINING COLOR TRANSPARENCY TO $22 \mathrm{GeV} / \mathrm{c}$

Spokespersons: A.S. Carroll [Brookhaven], S. Heppelmann [Penn State U. ]

E-mail contact carroll1@bnl.gov, heppel@phys.psu.edu,

heppelmann@bnldag.bnl.gov

$W W W$ Home-page

http://www.phys.psu.edu/LEPS/EVA/eva.htm

BNL-854 (Completed data-taking Jun 1991)

ANTIPROTON-NUCLEUS INTERACTIONS AT 5-10

$\mathrm{GeV} / \mathrm{c}$

Spokesperson: B.E. Bonner [Rice U.]

E-mail contact bonner@physics.rice.edu
BNL-859 (Completed data-taking 1992)

STUDIES OF HIGH DENSITY BARYON MATTER FROM EXTENDED MEASUREMENTS OF PARTICLE MOMENTUM DISTRIBUTIONS AND FROM HIGH PRECISION TWO-PARTICLE CORRELATIONS

Spokespersons: Louis P. Remsberg [Brookhaven], William A. Zajc [Columbia U.\}, Robert J. Ledoux [MIT]

E-mail contact remsberg@nc6.chm.bnl.gov, zajc@nevis.nevis.columbia.ed u

BNL-863 (Completed data-taking Sep 1993)

PARTICLE PRODUCTION AND NUCLEAR FRAGMENTATION IN COLLISIONS OF HEAVY IONS IN NUCLEAR TARGETS AT AGS ENERGIES

By EMU01 Collaboration

Spokespersons: Ingvar Otterlund [Lund U.], R.Jeffrey Wilkes [Washington U., Seattle]

E-mail contact ingvar.ot terlund@kosufy.lu.se,

kaj.soderstrom@kosufy.lu.se, wilkes@phys.washington.edu WWW Home-page http://marge.phys.washington.edu/emu01/

BNL-864 (In preparation)

PRODUCTION OF RARE COMPOSITE OBJECXS IN RELATIVISTIC HEAVY ION COLLISIONS

Spokespersons: Richard D. Majka, Jack Sandweiss [Yalc U.]

E-mail contact jack.sandweiss@yale.edu

WWW Home-page

http://rhic2.physics.wayne.edu/le864/e864.html

BNL-866 (Completed data-taking Jan 1996)

STUDIES OF PARTICLE PRODUCTION AT HXGH

BARYON DENSITY USING THE AU BEAM

By E-802 Collaboration

Spokespersons: Chellis Chasman [Brookhaven], Steve G. Steadman [MIT], Hideki Hamagaki [Tokyo U., INS]

E-mail contact chasman@hio.hirg.bnl.gov, sgs@mitlns.mit.edu, hamagaki@insuty.ins.u-tokyo.ac.jp

WWW Home-page

http://marie.mit.edu/server/e866/E866Main.html

BNL-868 (Completed data-taking Dec 1995)

INTERACTIONS OF $14.1 \mathrm{GeV} /$ NUCLEON NUCLEI FROM ${ }^{16}$ O TO ${ }^{197}$ Au IN LIGHT AND HEAVY TARGETS By KLMM Collaboration

Spokesperson: C.J. Waddington [Minnesota U.]

E-mail contact waddington $@$ uhn.spa.umn.edu

BNL-869

MEASUREMENT OF FRAGMENT YIELDS FOR 14

GeV/NUCLEON $A u+X$ COLLISIONS

By UHIC Collaboration

Spokesperson: C.Jake Waddington [Minnesota U.]

E-mail contact waddington@uhn.spa.umn.edu

WWW Home-page http://www.srl.caltech.edu/uhic/

BNL-874 (Completed data-taking Jun 1993)

KAON-NUCLEUS QUASIELASTIC AND ELASTIC SCATTERING

Spokespersons: Robert E. Chrien [Brookhaven], Roy Jerome Peterson [Colorado U.], E.V. Hungerford [Houston U.]

E-mail contact chrien@bnl.gov, peterson@spectr.colorado.edu, hunger@uh.edu 


\section{SUMMARIES OF BROOKHAVEN EXPERIMENTS}

BNL-875 (Completed data-taking Aug 1993)

STUDY OF PARTICLE PRODUCTION AND NUCLEAR FRAGMENTATION IN RELATIVISTIC HEAVY-ION COLLISIONS IN NUCLEAR EMULSIONS

Spokesperson: P.L. Jain [SUNY, Buffalo]

E-mail contact phyjain@ubvms.cc.buffalo.edu

BNL-877 (Completed data-taking Dec 1995)

STUDY OF RELATIVISTIC NUCLEAR COLLISIONS WITH HEAVY BEAMS USING THE E814 CALORIMETRY AND MODIFIED FORWARD SPECTROMETER Spokesperson: Peter Braun-Munzinger [SUNY, Stony Brook]

E-mail contact pbm@skipper.physics.sunysb.edu

WWW Home-page

http://skipper.physics.sunysb.edu/ e877/Home.html

BNL-878 (Completed data-taking Oct 1993)

INVESTIGATION OF ANTINUCLEUS PRODUCTION AND SEARCH FOR NEW PARTICLES IN NUCLEUSNUCLEUS COLLISIONS AT THE AGS

Spokesperson: H.J. Crawford [UC, Berkeley, Space Sci.]

E-mail contact hjcrawford@lbl.gov, crawford@bnldag.bnl.gov WWW Home-page http://aquila.lbi.gov/bni878/home_e878.html

BNL-882 (Completed data-taking Oct 1994)

SEARCH FOR PARTICLES WITH $|Z|>3$ AND

NEGATIVE CHARGE OR LARGE $A / Z$ PRODUCED IN CENTRAL NUCLEUS-NUCLEUS COLLISIONS

Spokesperson: P.Buford Price [UC, Berkeley]

E-mail contact pbprice@lbl.gov, yudong@physics.berkeley.edu

BNL-885 (In preparation)

EXPERIMENT TO DETECT $\Lambda$ HYPERNUCLEI

Spokespersons: M. May [Brookhaven], G.B. Franklin [Carnegie Mellon U.], Charles A. Davis [Manitoba U. and TRIUMF]

E-mail contact may2@bnl.gov, franklin@ernest.phys.cmu.edu, cymru@triumf.ca

BNL-886 (Completed data-taking Oct 1993)

SEARCH FOR NEW PARTICLES IN NUCLEUSNUCLEUS COLLISIONS

Spokespersons: Kenichi Imai [Kyoto U.], Philip H. Pile [Brookhaven], G. Diebold [Yale U.]

$\underline{\text { E-mail contact }}$ imai@kekvax.kek.jp, pile@bnl.gov

BNL-887 (Completed data-taking 1995)

DO NARROW $\Sigma$ HYPERNUCLEAR STATES EXIST?

Spokespersons: Reyad Sawafta [Brookhaven], Kenneth H. Hicks [Ohio U.]

E-mail contact sawafta@bnldag.bnl.gov,

hicks@ouvaxa.cats.ohiou.edu

BNL-888 (Completed datartaking Jul 1992)

SEARCH FOR THE $H$ DIBARYON

Spokespersons: R.D. Cousins [UCLA], Alan J. Schwartz [Princeton U.f

E-mail contact cousins@bnldag.bnl.gov, schwartz@puphep.princeton.edu
BNL-890 (Completed data-taking Jun 1995)

TEST OF CHARGE SYMMETRY IN $\eta$ PRODUCTION ON DEUTERIUM

Spokespersons: Robert E. Chrien [Brookhaven], J.C. Peng [Los Alamos], Bernard M.K. Nefkens [UCLA]

E-mail contact chrien@bnl.gov, peng@lanl.gov, bnefkens@uclapp.physics.ucla.edu

WWW Home-page

http://ucla5.phy.bnl.gov/htdocs/e890/e890.html

BNL-891 (In preparation)

A SEARCH FOR QUARK MATTER (QGP) AND OTHER NEW PHENOMENA UTILIZING AU Au COLLISIONS AT THE AGS

Spokesperson: E.D. Platner [Brookhaven]

E-mail contact platner@bnldag.bnl.gov

WWW Home-page http://www.phy.bnl.gov

/usr1/people/e891/doc/www/welcome_e891.html

BNL-892 (In preparation)

ELECTRON CAPTURE FROM PAIR PRODUCTION

Spokesperson: Harvey Gould [LBL, Berkeley]

E-mail contact hagould@lbl.gov

WWW Home-page http://www.ags.bnl.gov/ - e892/

BNL-895 (Taking data)

EXCLUSIVE STUDY OF NUCLEAR COLLISIONS AT THE AGS

Spokesperson: Gulshan Rai [LBL, Berkeley]

E-mail contact grai@lbl.gov

WWW Home-page http://cnr2.kent.edu/ e895/

BNL-896 (In preparation)

SEARCH FOR A SHORT-LIVED $H_{0}$ DIBARYON AND SHORT-LIVED STRANGE MATTER, AND STUDY OF HYPERON PRODUCTION IN $11.6 \mathrm{~A} \mathrm{GeV} / c \mathrm{Au} \mathrm{Au}$ COLLISIONS

Spokespersons: Henry J. Crawford [UC, Berkeley, Space Sci.], Tim J. Hallman [UCLA]

E-mail contact tjhallman@lbl.gov, hjcrawford@lbl.gov, crawford@bnldag.bnl.gov

WWW Home-page http://aquila.lbl.gov/bn1896/home_e896.html

BNL-900 (Completed data-taking Apr 1996)

ENERGY DISSIPATION AND MULTIFRAGMENTATION IN $p+A$ REACTIONS BETWEEN 2 AND $24 \mathrm{GeV} / c$ Spokespersons: Kris Kwiatkowski, Victor E. Viola [Indiana U.] E-mail contact kwiat@iucf.indiana.cdu, vicv@iucf.indiana.edu

\section{BNL-905 (Taking data)}

SEARCH FOR A $\Sigma$ HYPERNUCLEAR BOUND STATE IN ${ }^{4} \mathrm{He}\left(K^{-}, \pi^{ \pm}\right)$REACTIONS

Spokesperson: Tomofumi Nagae [Tokyo U., INS]

E-mazl contact tomofumi.nagae@kek.jp 


\section{SUMMARIES OF BROOKHAVEN EXPERIMENTS}

BNL-906 (In preparation)
EXPERIMENT TO DETECT DOUBLE-A HYPER-
NUCLEI BY OBSERVING CHARACTERISTIC $\pi^{-}$
MESONIC DECAY
Spokespersons: Robert Chrien [Brookhaven], Tomokazu Fukuda
[Tokyo U., INS]
E-mail contact chrien@bnl.gov, fukuda@insie1.ins.u-tokyo.ac.jp

BNL-907 (Taking data)

INVESTIGATION OF LIGHT HYPERNUCLEI USING $\left(K^{-}\right.$[stop], $\left.\pi^{0}\right)$ REACTION

Spokespersons: Ed V. Hungerford [Houston U.], Jen-Chieh Peng [Los Alamos]

E-mail contact hunger@uh.edu, peng@lanl.gov

BNL-910 (Taking data)

FACILITY TO STUDY PROTON-NUCLEUS AND

HEAVY ION COLLISIONS USING A LARGE ACCEPTANCE DETECTOR WITH PARTICLE IDENTIFICATION CAPABILITIES

Spokesperson: Brian A. Cole [Columbia U.]

E-mail contact cole@nevis.columbia.edu

WWW Home-page

http://nevis1.nevis.columbia.edu/heavyion/e910/

BNL-917 (In preparation)

HIGH DENSITY BARYON MATTER USING RARE PROBES

Spokespersons: Alice Mignerey [Maryland U.], R.K. Seto [UC, Riverside]

E-mail contact richard.seto@ucr.edu

\section{BNL Future Plans}

The physics program at the Lab is centered around the AGS and RHIC facilities. For the next few years, the AGS proton program will continue to emphasize the search for rare and forbidden $K$ decays, the measurement of $g-2$ for the muon, and studies of exotic mesons in the Multi-Particle Spectrometer. The heavy ion program will emphasize the search for the (strangeness 2) $H$ dibaryon and searches for strange baryonic fragments with anomalous charge/mass ratios (strangelets). An AGS-2000 Workshop took place in the spring of 1996. Its focus was on the competitive, leading-edge physics which can make use of the $10^{14}$ protons per pulse which will be available at the AGS. Among the experiments under consideration were a study of $K^{0} \rightarrow \pi^{0} \nu \bar{\nu}$ $\mu \rightarrow e$ conversion, polarization in $K_{\mu 3}$, and searches for low-mass gluino-gluino bound states. It is anticipated that the core of the $\Lambda$ GS program beyond the year 2000 will develop from these and other Workshop efforts. The RHIC construction project is well underway. The current schedule calls for the first circulating and accelerating beams by the beginning of 1999 , and the first physics runs later that year. 


\section{SUMMARIES OF CEBAF EXPERIMENTS}

\section{Selected CEBAF Experiments}

\section{CEBAF-89-024}

(Proposed 1987, Approved 1989, In preparation)

\section{RADIATIVE DECAYS OF LOW-LYING HYPERONS}

CLAS COLLABORATION

RICE U - S Ahmad, B E Bonner, G.S Mutchler ( $\sqrt{ }$ Spokesperson), $S$ Taylor

WILLIAM AND MARY COLL - M Eckhause, A D Hancock,

J R Kane, Y N Kuang, R E Welsh

Accelerator CEBAF Detector CLAS

Reactions

$$
\gamma p \rightarrow K^{+} Y^{*} \text { (unspec) } \quad 1.40-3.02 \mathrm{GeV}\left(\mathrm{E}_{\mathrm{lab}}\right)
$$

Particles studied $\Lambda\left(1405 S_{01}\right), \Lambda\left(1520 D_{03}\right), \Sigma\left(1385 P_{13}\right)^{\circ}$

Brief descriptzon Measures the electromagnetic branching ratios of low-lying excited hyperons, $\Lambda(1405), \Lambda(1520)$ and $\Sigma^{0}(1385)$. Uses the CLAS detector, a superconducting toroidal spectrometer with drift chambers and TOF scintillators. Determines the four-momentum of the excited hyperon from the energy of the tagged photon and $K^{+}$momentum. The four-momentum of $\Lambda$ (from the $Y^{*} \rightarrow \Lambda \gamma$ decay) can be reconstructed from the proton and $\pi^{-}$momentum. A good mass resolution with CLAS allows the suppression of the background due to $\pi^{0}$ decays. Uses a tagged photon beam and an $\mathrm{LH} 2$ target. Approved for 60 days of running in Hall B. Expected to run in Summer and Fall 1997.

Related experiments CEBAF-89-004, BNL-811

E-mail contact mutchler@physics.rice.edu

\section{CEBAF-89-038}

(Proposed Oct 1989, Approved May 1990, In preparation)

MEASUREMENT OF $p\left(e, e^{\prime} \pi^{+}\right) n, p\left(e, e^{\prime} p\right) \pi^{0}$, AND $n\left(e, e^{\prime} \pi^{-}\right) p$ IN THE SECOND AND THIRD RESONANCE REGIONS

N* COLLABORATION

CEBAF - W Brooks, V D Burkert ( $V$ Spokesperson), D Joyce,

B A Mecking, M Mestayer, B B Niczyporuk, E Smith,

A Yegneswaran

CHRISTOPIrER NEWPORT U - D Doughty, D Heddle, Z J Li

DUKE U - H R Weller

FLORIDA STATE U - L C Dennis, P Dragovitsch, K W Kemper

FRASCATI - N Bianchi, G P Capitani, E De Sanctis, P Levi-

Sandri, V Muccifora, E Polli, A R Reolon, P Rossi

INFN, GENOA - M Anghinolfi, P Corvisiero, G Gervino,

L Mazzaschi, V Mokeev, G Ricco, M Ripani, M Sanzone,

M Taiuti, A Zucchiatti

GEORGE MASON U - B J Lieb

JAMES MADISON U - K Giovanetti

KENT STATE U -.. D Keane, D M Manley

PIT'TSBURGH U - S A Dytman

RENSSELAER POLY - G S Adams, N C Mukhopadhyay, P Stoler

VIRGINIA U -. D B Day, J S McCarthy, R C Minehart

( $\checkmark$ Spokesperson), D Počanić, O A Rondon-Aramayo, R Sealock,

L C Smith, S T Thornton, H J Weber

VIRGINIA STATE U - C E Stronach

VIRGINIA TECH - $\mathrm{R}$ A Andt, D A Jenkins, L D Roper

WILLIAM AND MARY COLL - C E Carlson, H O Funsten,

$T$ Y Tung

CONNECTICUT U - M Gai ( $\checkmark$ Spokesperson)

Accelerator CEBAF Detector CLAS

Reactions

$$
\begin{array}{lc}
e^{-} p \rightarrow e^{-} p \pi^{0} & 2.0,4.0 \mathrm{GeV}\left(\mathrm{T}_{\mathrm{lab}}\right) \\
e^{-} p \rightarrow e^{-} n \pi^{+} & " \\
e^{-} p \rightarrow e^{-} p p \pi^{-} & "
\end{array}
$$

Particles studied $\quad N\left(1535 S_{11}\right), N\left(1520 D_{13}\right), N\left(1680 F_{15}\right)$, $N\left(1440 P_{11}\right)$

Brief description The experiment will measure transition formfactors to nucleon excited states in the mass region from 1350 to $1800 \mathrm{MeV} / \mathrm{c}^{2}$. Scheduled to run in Hall $\mathrm{B}$ in 1997.

Related experiments CEBAF-89-037, CEBAF-89-040, CEBAF89-042, CEBAF-89-043, CEBAF-91-002, CEBAF-93-036

E-mail contact burkert@cebaf.gov, minehart@virginia.edu, gai@uconnvm.uconn.edu

\section{CEBAF-89-039}

(Proposed Oct 1989, Approved 1989, In preparation)

AMPLITUDES FOR THE $N\left(1535 S_{11}\right)$ AND $N\left(1710 P_{11}\right)$ RESONANCES FROM THE $e p \rightarrow e p \eta$ SCATTERING

CLAS COLLABORATION

CEBAF - V D Burkert, D Cords, D Joyce, B A Mecking,

M Mestayer, B B Niczyporuk, E Smith, A Yegneswaran CHRISTOPHER NEWPORT U - D Doughty, L Elouadrhiri, $D$ Heddle

DUKE U - R Chasteler, D R Tilley, H Weller

FLORIDA STATE U - Ł C Dennis, P Dragovitsch, K W Kemper GEORGE MASON U - B J Lieb

FRASCATI - N Bianchi, G P Capitani, E De Sanctis, P Levi-

Sandri, V Muccifora, E Polli, A R Reolon, P Rossi

GENOA U \& INFN, GENOA - M Anghinolf, P Corvisiero,

G Gervino, L Mazzaschi, V I Mokeev, G Ricco, M Ripani,

$M$ Sanzone, $M$ Taiuti, $\Lambda$ Zucchiatti

JAMES MADISON U - K Giovanetti ( $\checkmark$ Spokesperson)

KENT STATE U - D M Manley

PITTSBURGH U - S A Dytman ( $\checkmark$ Spokesperson), J Mueller,

D Tedeschi, R Thompson

RENSSELAER POLY - G S Adams, N C Mukhopadhyay,

J Napolitano, J Pricc, P Stoler

VIRGINIA U - D B Day, R Marshall, J S McCarthy,

R C Minehart, D Počanić, O A Rondon-Aramayo, R Sealock,

$\mathrm{S}$ T Thornton, $\mathrm{H} J$ Weber

VIRGINIA STATE U - C E Stronach

VIRGINIA TECH - R A Arndt, D A Jenkins, L D Roper

WILLIAM AND MARY COLL - C E Carlson, $\mathrm{H}$ O Funsten,

T Y Tung

YALE U - M Gai

Accelerator CEBAF Detector CLAS

Reactions

$$
e^{-} p \rightarrow e^{-} p \eta \quad 2,4 \mathrm{GeV}\left(\mathrm{T}_{\mathrm{lab}}\right)
$$

Particles studied $N\left(1535 S_{11}\right), N\left(1710 P_{11}\right), N^{*}$ (unspec)

Brief description Covers the total center-of-mass energies, $\mathrm{W}$

from 1.35 to $1.9 \mathrm{GeV}$ for the second resonance region, and from 1.49 to $1.9 \mathrm{GcV}$ for the $\eta$ channel. Uses a simple hydrogen gas target. Scheduled to run in Hall B.

E-mail contact giovankl@vax $1 . j m u . e d u$,

dytman@vms.cis.pitt.edu

WWW Home-page http://www.cebaf.gov/clas/CLAS.html

\section{CEBAF-89-043}

(Proposed Oct 1989, Approved 1989, In preparation)

MEASUREMENTS OF THE ELECTROPRODUCTION OF THE $\Lambda, \Lambda(1520)$, AND $f_{0}(975)$ VIA THE $K^{+} K^{-} p$ AND THE $K^{+} \pi^{-} p$ FINAL STATES

ABILENE CHRISTIAN U - D Isenhower, M Sadler MI'I', BATES LINEAR ACCELERATOR -- L Ghedira

CEBAF - V D Burkert, D Joyce, B A Mecking, M Mestayer,

B B Niczyporuk, E Smith, R R Whitney, A Yegneswaran

CARNEGIE MELLON U - $R A$ Schumacher

CHRISTOPHER NEWPORX U - D Doughty

FLORIDA STATE U - L C Dennis (Spokesperson), K W Kemper GEORGE MASON U - B J Lieb

GEORGE WASHINGTON U - A Mokhtar

JAMES MADISON U K Giovanetti 


\section{SUMMARIES OF CEBAF EXPERIMENTS}

KANSAS STATE U - T R Donoghue

KENT STATE U - D Keane, D M Manley

MIT - W Y Kim

PITTSBURGH U - S A Dytman

RENSSELAER POLY - G S Adams, N C Mukhopadhyay, P Stoler

VIRGINIA U - D B Day, R Marshall, J S McCarthy,

$\mathrm{R} C$ Minehart, O A Rondon-Aramayo, $\mathrm{R}$ Sealock, $\mathrm{S} T$ Thornton, H J Weber

VIRGINIA STATE $U-C$ E Stronach

VIRGINIA TECH - $\mathrm{R}$ A Arndt, D A Jenkins, L D Roper

WILLIAM AND MARY COLL - C E Carlson, H O Funsten

(Spokesperson), C F Perdrisat

YALE U - M Ga

Accelerator CEBAF Detector CLAS

Reactions

$e^{-} p \rightarrow e^{-} \mathrm{X} \quad 4 \mathrm{GeV}\left(\mathrm{T}_{\mathrm{lab}}\right)$

Particles studied $f_{0}(980), \Lambda\left(1520 D_{03}\right)$

Brief description Studies exclusive electroproduction and

charged hadronic decay of the $f_{0}(980)$ resonance and low-mass

$\Lambda$ 's. Scheduled to run in Hall $B$.

$\underline{\text { E-mail contact }}$ larry@fsulcd.physics.fsu.edu, funsten@cebaf.gov

\section{CEBAF-91-002}

(Proposed Sep 1991, Approved Nov 1991, Jan 1995, In preparation)

THE STUDY OF EXCITED BARYONS AT HIGH MOMENTUM TRANSFER WITH THE CLAS SPECTROMETER

$N^{*}$ COLLABORATION

CEBAF - VD Burkert ( $\checkmark$ Spokesperson), B A Mecking,

M Mestayer, B B Niczyporuk, E Smith, B Wojtsekhowski,

A Yegneswaran

CHRISTOPHER NEWPORT U - D Doughty, D Heddle, $Z J \mathrm{Li}$

CONNECTICUT U - M Gai

DUKE U - R Chasteler, D R Tilley, H R Weller

FLORIDA STATE $U-L C$ Dennis, P Dragovitsch

FRASCATI - N Bianchi, G P Capitani, E De Sanctis, P Levi-

Sandri, V Muccifora, E Polli, A R Reolon, P Rossi

INFN, GENOA .. M Anghinolfi, P Corvisiero, G Gervino,

L Mazzaschi, V Mokcev, G Ricco, M Ripani, M Sanzone,

$M$ Tajuti ( $\checkmark$ Spokesperson), A Zucchiatti

GEORGE MASON U - B J Lieb

HAMPTON U - K Beard

JAMES MADISON U - K Giovanetti

KENT STATE U - D M Manley

PITTSBURGH U - S A Dytman

RENSSELAER POLY - G S Adams, N C Mukhopadhyay,

$J$ Napolitano, P Stoler ( $\checkmark$ Spokesperson)

VIRGINIA U - D Crabb, D B Day, R Marshall, J S McCarthy,

R C Minehart, D Počanić, O A Rondon-Aramayo, R Sealock,

L C Smith, S T Thornton, H Weber

WILLIAM AND MARY COLL - C E Carison, A Coleman,

H O Funsten, $T$ Y Tung

Accelerator CEBAF Detector CLAS

Reactions

$$
\begin{array}{lc}
e^{-} p \rightarrow e^{-} p \pi^{0} & 6.0 \mathrm{GeV}\left(\mathrm{T}_{\mathrm{lab}}\right) \\
e^{-} p \rightarrow e^{-} p \eta & " \\
e^{-} p \rightarrow e^{-}{ }_{n}^{+} & "
\end{array}
$$

Paricies studied $\Delta\left(1232 P_{33}\right), N\left(1440 P_{11}\right), N\left(1535 S_{11}\right)$, $N\left(1680 F_{15}\right)$

Brief description Studies the transition form-factors of prominent resonances, $\Delta\left(1232 P_{33}\right), N\left(1440 P_{11}\right), N\left(1535 S_{11}\right)$, and $N\left(1680 F_{15}\right)$ at high momentum transfers, in the transition region where constituent-quark models are expected to become less relevant and gluons and current-quarks are believed to play a more active role. Scheduled to run in Hall B in 1996/97.

Related experiments CEBAF-94-014

E-mail contact stoler@rpi.edu, burkert@cebaf.gov

\section{CEBAF-91-008}

(Proposed Sep 1991, Approved Jan 1992, In preparation)

\section{PHOTOPRODUCTION OF $\eta$ AND $\eta^{\prime}$ MESONS}

CLAS COLLABORATION

ARIZONA STATE U - B G Ritchie ( $\checkmark$ Spokesperson)

CATHOLIC U - H Crannell, J T O'Brien, D I Sober

CEBAF - B A Mecking

FLORIDA STATE U - L C Dennis

GEORGETOWN U - J Lambert

GEORGE WASHINGTON U - B L Berman, W J Briscoe,

K Dhuga, W R Dodge

BOŠKOVIĈ INST, ZAGREB - I Šlaus

SOUTH CAROLINA U - C Djalali, B M Preedom, A Tam,

S Whisnant

UCLA - B M K Nefkens

Accelerator CEBAF Detector CLAS

$\underline{\text { Reactions }}$

$$
\begin{array}{lc}
\gamma p \rightarrow \eta p & 0.65-2.25 \mathrm{GeV}\left(\mathrm{E}_{\mathrm{lab}}\right) \\
\gamma p \rightarrow \eta^{\prime} p & "
\end{array}
$$

Particles studied $\eta, \eta^{\prime}$

Brief description Measures the differential cross-section for the photoproduction of $\eta$ and $\eta^{\prime}$ mesons using the Hall-B bremsstrahlung photon tagger and the CLAS spectrometer. The target is a liquid hydrogen cell. Identification of the $\eta$ and $\eta^{\prime}$ is made by detection of the recoil proton in the CLAS. Studies the properties of $\eta, \eta^{\prime}, N\left(1535 S_{12}\right)$, and $N\left(1710 P_{11}\right)$. Scheduled to run in Hall $\mathrm{B}$.

Related experiments CEBAF-89-039, CEBAF-89-045, CEBAF93-008, CEBAF-93-017

E-mail contact barry.ritchie@asu.edu

\section{CEBAF-91-011}

(Proposed Oct 1991, Approved Dec 1993, In preparation)

HIGH PRECISION SEPARATION OF POLARIZED STRUCTURE FUNCTIONS IN ELECTROPRODUCTION OF THE $\triangle$ AND ROPER RESONANCES

CAI STATE, LA - M B Epstein

CEBAF -. J P Chen, J J LeRose, J H Mitchell, S Nanda, A Saha INFN, ROME - E Cisbani, $R$ de Leo, F Ghio, $M$ Jodice,

G M Urciuoli

INFN, LECCE - R Perrino

MARYLAND U - J J Kelly, P Markowitz

MIT - W Bertozzi, D Dale, S Gilad, A Sarty

NIKHEF, AMSTERDAM - H P Blok

OLD DOMINION U - P E Ulmer, L B Weinstein

RUTGERS U - R Gilman, C Glashausser, G Kumbartzki,

R Ransome, P M Rutt

SACLAY - J Y Mougey

NEW HAMPSHIRE $U-J$ Calarco

ROME U, TORVERGATA - S Frullani ( $\checkmark$ Spokesperson), F Garibaldi

VIRGINIA U - D H Barkhuff. R Lourie ( $\checkmark$ Spokesperson), B Milbrath, S Van Verst

WILLIAM AND MARY COLL - J M Finn, M Jones, C F Perdrisat

Accelerator CEBAF Detector Spectrometer

Reactions Polarized beam

$$
e^{-} p \rightarrow e^{-} p \pi^{0} \quad 3.2 \mathrm{GeV} / c
$$

Particles studied $\Delta\left(1232 P_{33}\right)$

Brief description Studies the six structure functions in the $N-\Delta$ transition as a function of $\theta_{\mathrm{cm}}$. The measurement is performed at $Q^{2}=1.0(\mathrm{GeV} / \mathrm{c})^{2}$, at $6 \mathrm{c}$. $\mathrm{m}$. angles, which allows a standalone multipole analysis of the amplitudes. Uses high-resolution spectrometers and a focal plane polarimeter. The target is liquid hydrogen. Scheduled to run in Fall A.

Related experiments CEBAF-89-037, -89-042,-93-036

E-mail contact frullani@sanita.infn.it, lourie@virginia.edu 


\section{SUMMARIES OF CEBAF EXPERIMENTS}

\section{CEBAF-91-023}

(Proposed Oct 1991, Approved May 1992, Jan 1995, In preparation)

MEASUREMENT OF POLARIZED STRUCTURE FUNCTIONS IN INELASTIC ELECTRON-PROTON SCATTERING USING THE CEBAF LARGE ACCEPTANCE SPECTROMETER

N* COLLABORATION

CEBAF - W Brooks, VD Burkert ( $\checkmark$ Spokesperson),

A Freyberger, B A Mecking, M Mestayer, B B Niczyporuk, E Smith, A Yegneswaran

CHRISTOPHER NEWPORT U - D Doughty, L Elouadrhiri, D Heddle

DUKE U - M Spraeker, H R Weller

FLORIDA STATE U - L C Dennis, P Dragovitsch, K W Kemper

FRASCATI - N Bianchi, G P Capitani, E De Sanctis, P LeviSandri, V Muccifora, E Polli, A R Reolon, P Rossi

INFN, GENOA - M Anghinolf, P Corvisiero, G Gervino,

L Mazzaschi, V Mokeev, G Ricco, M Ripani, M Sanzone,

M Taiuti, A Zucchiatti

HAMPTON U - K B Beard

JAMES MADISON U - K Giovanetti

KENT STATE U - D Keane, D M Manley

OLD DOMINION U - S Kuhn

PITTSBURGH U - S A Dytman

RENSSELAER POLY - G S Adams, N C Mukhopadhyay, P Stoler

VIRGINIA U - D G Crabb ( $\checkmark$ Spokesperson), D B Day,

J S McCarthy, R C Minehart ( $\checkmark$ Spokesperson), O A Rondon-

Aramayo, R Sealock, L C Smith, S T Thornton, H J Weber

VIRGINIA STATE U - C E Stronach

VIRGINIA TECFY - R A Arndt, D A Jenkins, L D Roper

WILLIAM AND MARY COLL - A Coleman, H O Funsten,

T Y Tung

YALE U - M Gai

Accelerator CEBAF Detector CLAS

Reactions Polarized beam and target

$$
e^{-} p \rightarrow e^{-\mathrm{X}} \quad 1.2-4.0,6.0 \mathrm{GeV}\left(\mathrm{T}_{\text {lab }}\right)
$$

Particles studied $N\left(1440 P_{11}\right)$

Brief description Measures the inclusive polarized structure

functions, $A_{1}$ and $A_{2}$, in the range $0.2 \leq Q^{2} \leq 2.5(\mathrm{GeV} / \mathrm{c})^{2}$ and $1.1<W<2.5 \mathrm{GeV}$. Uses polarized $\mathrm{NH}_{3}$ target. Scheduled to run in Hall B in 1998.

Related experiments CEBAF-93-009

E-mail contact burkert@cebaf.gov, dcrabb@virginia.edu, minehart@virginia.edu

\section{CEBAF-91-024}

(Proposed Oct 1991, Approved Dec 1991, In preparation)

SEARCH FOR MISSING RESONANCES IN $T H E$ ELECTROPRODUCTION OF $\omega$ MESONS

N* COLLABORATION

CEBAF - W Brooks, V D Burkert (Spokesperson), D Joyce,

B A Mecking (Spokesperson), M Mestayer, B B Niczyporuk, E Smith, A Yegneswaran

CHRISTOPHER NEWPORT U - D Doughty, D Heddle, Z J Li

FLORIDA STATE U - L C Dennis

FRASCATI - N Bianchi, G P Capitani, E De Sanctis, P Levi-

Sandri, V Muccifora, E Polli, A R Reolon, $P$ Rossi

INFN, GENOA - M Anghinolfi, P Corvisiero, G Gervino,

L Mazzaschi, V Mokeev, G Ricco, M Ripani, M Sanzone,

M Taiuti, A Zucchiatti

GEORGE MASON U - B J Lieb

HAMPTON U - K B Beard

JAMES MADISON U - K Giovanetti

KENT STATE U - D M Manley (Spokesperson)

PITTSBURGH U - S A Dytman

RENSSELAER POLY - G S Adams, N C Mukhopadhyay, P Stoler
VIRGINIA U - D G Crabb, D B Day, R Marshall, J S McCarthy R C Minehart, D Poćanić, O A Rondon-Aramayo, $R$ Sealock, L C Smith, S T Thornton, H J Weber

VIRGINIA STATE U - C E Stronach

WILLIAM AND MARY COLL - C E Carlson, H O Funsten

(Spokesperson), $\mathrm{T}$ Y Tung

YALE U - M Gai

Accelerator CEBAF Detector CLAS

Reactions

$$
e^{-} p \rightarrow e^{-} p \pi^{+} \pi^{-} \mathrm{X} \quad 1.6,2.4,4.0 \mathrm{GeV}\left(\mathrm{T}_{\mathrm{lab}}\right)
$$

Brief description The experiment is aimed at searching for 'missing' 3-quark baryon states in the mass range from 1.7 to $2.2 \mathrm{GeV} / c^{2}$ in the $p \omega$ decay channel. Scheduled to run in Hall $\mathrm{B}$ in $1996 / 97$.

E-mail contact burkert@cebaf.gov, mecking@cebaf.gov, manley@ksuvxa.kent.edu, funsten@cebaf.gov

\section{CEBAF-93-006}

(Proposed Apr 1993, Approved Jun 1993, In preparation) TWO PION DECAY OF EXECTROPRODUCED LIGHT QUARK BARYON RESONANCES

N* COLLABORATION

CEBAF - W Brooks, V D Burkert ( $\checkmark$ Spokesperson),

B A Mecking, B B Niczyporuk, E Smith, A Yegneswaran CHRISTOPHER NEWPORT U - D Doughty, D Heddle DUKE U - R Chasteler, $H R$ Weller

FLORIDA STATE U - L C Dennis, P Dragovitsch

FRASCATI - N Bianchi, G P Capitani, E De Sanctis, P Levi-

Sandri, V Muccifora, E Polli, A R Reolon, P Rossi

INFN, GENOA - M Anghinolf, P Corvisiero, V Mokeev, G Ricco,

$M$ Ripani ( $\checkmark$ Spokesperson), M Sanzone, M Taiuti, A Zucchiatti

GEORGE MASON U - B J Lieb

HAMPTON U - K B Beard

JAMES MADISON U - K Giovanetti

KENT STATE U - D M Manley

NORTH CAROLINA STATE U - D R Tilley

PITTSBURGH U - S A Dytman

RENSSELAER POLY - G S Adams, N C Mukhopadhyay,

$J$ Napolitano, $P$ Stoler

VIRGINIA U - D G Crabb, D B Day, R Marshall, J S McCarthy,

R C Minehart, D Počanić, O A Rondon-Aramayo, R Sealock,

L C Smith, S T Thornton, H J Weber

WILLIAM AND MARY COLL - C E Carlson, A Coleman.

H O Funsten, T Y Tung

YALE U - M Gai

Accelerator CEBAF Detector CLAS

Reactions

$e^{-} p \rightarrow e^{-}$nucleon pion pion $\mathrm{X}$

$1.6,2.4,4.0 \mathrm{GeV}$

$e^{-} n \rightarrow e^{-}$nucleon pion pion $\mathrm{X}$

( $\left.\mathrm{T}_{\text {lab }}\right)$

Brief description Studies some aspects of baryon spectra in the nonstrange sector, e.g., the form-factors of some poorly known states. It also looks for highly excited nucleon states around $1.6 \mathrm{GeV}$ (mostly $[56,0+]$ and $[70,1-]$ multiplets) and the 'missing' states around $2 \mathrm{GeV}$ (mostly $[56,2+]$ and $[70,2+]$ multiplets) predicted by quark models. Such states would strongly decay through $\Delta \pi$, and $\rho N$ channels, both giving a final state with two pions. Approved for 800 hours with hydrogen target and 400 hours with deutcrium target. Expected to run in Hall B in $1996 / 97$.

Related experiments CEBAF-91-024, CEBAF-91-002, CEBAF93-033

E-mail contact burkert@cebaf.gov, ripani@genova.infn.it WWW Home-page http://aiacehp.ge.infn.it/twopion.html 


\section{SUMMARIES OF CEBAF EXPERIMENTS}

\section{CEBAF-93-030}

(Proposed Apr 1993, Approved Jun 1993, In preparation)

MEASUREMENT OF THE STRUCTURE FUNCTIONS FOR KAON ELECTROPRODUCTION

\section{CLAS COLLABORATION}

CEBAF - M Mestayer ( $\checkmark$ Spokesperson), E Smith

CARNEGIE MELLON U - R Magahiz, R A Schumacher

CHRISTOPHER NEWPOR' U -- D Doughty

FLORIDA STATE U - S Capstick

GEORGE WASHINGTON U - C Bennhold

OHIO U - T Adami, R W Finlay, S Grimes, $\mathrm{K}$ H Hicks

( $\sqrt{ }$ Spokesperson), A Kumar, D S Onley, J Rapaport,

L E Wright

PITTSBURGH U - S A Dytman

RICHMOND U - P D Rubin

SOUTH CAROLINA U - A Tam

WILLIAM AND MARY COLL - H O Funsten

Accelerator CEBAF Detector CLAS

Reactions

$$
\begin{array}{ccc}
e^{-} p \rightarrow e^{-} K^{+} \Lambda & 2.4,3.2,4.0 \mathrm{GeV}\left(\mathrm{\Upsilon}_{\mathrm{lab}}\right) \\
e^{-} p \rightarrow e^{-} K^{+} \Sigma^{0} & \|
\end{array}
$$

Particles studied $N^{*}$ (unspec)

Brief description Measures $L, T, L T$, and $T T$ structure

functions for $Q^{2}$ between 1 and $2(\mathrm{GeV} / \mathrm{c})^{2}$ and $W$ between

1.8 and $2.2 \mathrm{GeV}$. Measures isospin dependence by comparing

$\Lambda$ and $\Sigma$ productions. Studies production ratio of hyperons up

to the $\Lambda(1520)$. Measures polarization of $\Lambda$. Searches for $N^{*}$

resonances which decay to hyperon $-K^{+}$final states. Scheduled to run in Hall B.

Journal papers NIM A323 (1992) 193, and IEEE TNS 39 (1992) 690.

E-mail contact mestayer@cebaf.gov,

hicks@ouvaxa.cats.ohiou.edu, khicks1@ohiot1.edu

\section{CEBAF-93-033}

(Proposed Apr 1993, Approved Jun 1993, In preparation)

\section{A SEARCH FOR MISSING BARYONS FORMED IN} $\gamma p \rightarrow p \pi^{+} \pi^{-}$USING THE CLAS DETECTOR

CLAS COLLABORATION

RENSSELAER POLY - G S Adams, J Napolitano

( $\checkmark$ Spokesperson), P Stoler, M Witkowski, B B Wojtsekhowski CARNEGIE MELLON U - R Schumacher

RICE U - G Mutchler

KENT STA'IE U - D M Manley

Accelerator CEBAF Detector CLAS

Reactions

$$
\gamma p \rightarrow p \pi^{+} \pi^{-} \quad 0.5-2.3 \mathrm{GeV}\left(\mathrm{E}_{\mathrm{lab}}\right)
$$

Particles studied $N^{*}$ (unspec)

Brief description Uses tagged photons and liquid hydrogen

target. Scheduled to run in Hall B in 1996/97.

Related experiments CEBAF-89-004, CEBAF-89-024

E-mail contact jimnap@rpi.edu

WWW Home-page http://www.cebaf.gov/clas/CLAS.html

\section{CEBAF-93-036}

(Proposed Apr 1993, Approved Jun 1993, In preparation)

MEASUREMENT OF SINGLE PION ELECTROPRODUCTION FROM THE PROTON WITH POLARIZED BEAM AND POLARIZED TARGET USING CLAS

N* COLLABORAIION

CEBAF - W Brooks. V D Burkert, D Joyce, B A Mecking,

B B Niczyporuk, E S Smith, A Yegneswaran
CHRISTOPHER NEWPORT $U$ - D Doughty, D Heddle, Z J Li DUKE U - R Chasteler ( $\checkmark$ Spokesperson), II R Weller ( $\checkmark$ Spokesperson)

FLORIDA STATE U - L C Dennis, P Dragovitsch

FRASCATI - N Bianchi, G P Capitani, E De Sanctis, P LeviSandri, V Muccifora, E Polli, A R Reolon, P Rossi

INFN, GENOA - M Anghinolfi, P Corvisiero, G Gervino,

L Mazzaschi, V Mokeev, G Ricco, M Ripani, M Sanzone, M Taiuti, A Zucchiatti

GEORGE MASON U - B I Licb

HAMPTON U - K B Beard

JAMES MADISON U - K Giovanetti

KENT STATE U - D M Manley

PITTSBURGH U - S A Dytman

RENSSELAER POLY - G S Adams, N C Mukhopadhyay, P Stoler

VIRGINIA U - D G Crabb, D B Day, I S McCarthy,

RC Minehart ( $\checkmark$ Spokesperson), D Počanić, O A RondonAramayo, R Sealock, L C Smith, S T Thornton, H J Weber VIRGINIA STATE U - C E Stronach

WILLIAM AND MARY COLL - C E Carlson, H O Funsten

YALE U - M Gai

Accelerator CEBAF Detector CLAS

Reactions Polarized beam and target

$$
\begin{array}{cc}
e^{-} p \rightarrow e^{-} \pi^{+} n & 1.2-4.0 \mathrm{GeV}\left(\mathrm{T}_{\mathrm{lab}}\right) \\
e^{-} p \rightarrow e^{-} p \pi^{0} & "
\end{array}
$$

Particles studied $N\left(1440 P_{11}\right), N\left(1520 D_{13}\right), \Delta\left(1232 P_{33}\right)$

Bricf description Measures polarization asymmetries for exclusive single-pion channels. Provides information on the Roper resonance. Scheduled to run in Hall B in 1998/99.

Related experiments CEBAF-89-037, CEBAF-89-038, CEBAF89-040, CEBAF-89-042, CEBAF-89-043, CEBAF-91-002

E-mail contact bobber@tunl.tunl.duke.edu, weller@tunl.tunl.duke.edu,minchart@virginia.edu

\section{CEBAF-94-008}

(Proposed Apr 1994, Approved Jun 1994, In preparation)

\section{PHOTOPRODUCTION OF $\eta$ and $\eta^{\prime}$ MESONS FROM} DEUTERIUM

CLAS COLLABORATION

ARIZONA STATE U - B Ritchie ( $\checkmark$ Spokesperson)

CEBAF - B A Mecking

GEORGETOWN U - J Lambert

RICHMOND U - G P Gilfoyle, $\mathrm{R}$ W Major, M F Vineyard SOUTH CAROLINA U - C Djalali, B Preedom, S Whismant

Accelerator CEBAF Detector CLAS

$$
\begin{aligned}
& \text { Reactions } \\
& \gamma \text { deut } \rightarrow \eta \text { deut } \quad 0.63-1.52 \mathrm{GeV}\left(\mathrm{E}_{\mathrm{lab}}\right) \\
& \gamma \text { deut } \rightarrow \eta^{\prime} \text { deut }
\end{aligned}
$$

Particles studied $\quad \eta, \eta^{\prime}, N\left(1535 S_{11}\right), N\left(1710 P_{11}\right)$

Brief description Measures the differential cross-section for the coherent and incoherent photoproduction of $\eta$ and $\eta^{\prime}$ mesons on the deuteron for photon energies from 0.63 to $1.52 \mathrm{GeV}$. Uses a liquid deuterium target cell and a tagged photon beam. Provides information on the properties of $\eta, \eta^{\prime}$ and gives insight into the structure of related nucleon resonances. Studics the behavior of the mesons in the lightly bound two-nucleon systems. Scheduled to run in Hall B, for 23 days (simultaneously with CEBAF-89-045, and CEBAF-93-017).

Related experiments CEBAF-89-039, CEBAF-89-045, CEBAF93-008, CEBAF-93-017, CEBAF-91-008

E-mail contact barry.ritchie@asu.edu

\section{CEBAF-94-016}

(Proposed Apr 1994, Approved Jan 1995, In preparation)

MEASUREMENT OF RARE RADIATIVE DECAYS OF THE $\phi$ MESON 


\section{SUMMARIES OF CEBAF EXPERIMENTS}

CATHOLIC U - H Cramell, J O'Brien, D I Sober

CEBAF - V D Burkert, B Niczyporuk, E S Smith

INDIANA U - B Brabson, R Crittenden, A Dzierba

( $\checkmark$ Spokesperson), J Gunter, R Lindenbusch, D Rust, P Smith, $S$ Teige

MOSCOW, ITEP - M V Kossov

NOTRE DAME U - J J Kolata, J Losecco, A H Sanjari,

W D Shephard

RICE U - S Ahmad, B E Bonner, G Mutchler

RICHMOND U - G P Gilfoyle, P Rubin, M F Vineyard

RENSSELAER POLY - G S Adams, J J Napolitano ( $\sqrt{ } \mathrm{Co}$ spokesperson), M T Witkowski

VIRGINIA U - E Frlež, D Počanić

WILLIAM AND MARY COLL - D Armstrong

Accelerator CEBAF Detector Calorimeter

Reactions

$$
\gamma p \rightarrow \eta p \quad 4 \mathrm{GeV}\left(\mathrm{E}_{\mathrm{lab}}\right)
$$

Particles studied $\phi, \rho, \omega$

Brief description Uses the $4 \mathrm{GeV}$ tagged photon beam and a lead glass detector to study decays of the $\phi$ into all-photon final states. Expects to achieve the branching ratio sensitivity of about $10^{-5}$, which should be enough to observe the (yet unobserved) decays $\phi \rightarrow f_{0}(980) \gamma, \phi \rightarrow a_{0}(980) \gamma$, and $\phi \rightarrow \eta^{\prime} \gamma$, and to improve the measurement of other branching ratios of $\phi, \rho$ and $\omega$. Scheduled to run in Hall B, for 30 days.

Related experiments BNL-852

E-mail contact dzierba@indiana.edu, jimnap@rpi.edu WWW Home-page

http://www.cebaf.gov/physics/radphi/radphi.html

\section{CEBAF-94-109}

(Proposed Dec 1994, Approved Jan 1995, In preparation)

PHOTOPRODUCTION OF THE $\rho$ MESON FROM THE PROTON WITH LINEARLY POLARIZED PHOTONS

ARIZONA STATE $U-R$ Alarcon, J Comfort

CATHOLIC U - H Crannell, S Matthews, J O'Brien, D I Sober CEBAF - R R Whitney ( $\checkmark$ Spokesperson)

GEORGE WASHINGTON U - B L Berman, W I Briscoe,

P L Cole ( $\checkmark$ Spokesperson), J P Connelly ( $\checkmark$ Spokesperson),

K S Dhuga, L Murphy, S Rugari

KENT STATE U - D M Manley

OLD DOMINION U - C Hyde-Wright, W Roberts

RENSSELAER POLY - J J Napolitano

SACLAY - G Audit, M Guidal, F Kunne-Perrot, I M Laget,

C Marchand, B Saghai

FLORIDA STATE U, SCRI - S C Capstick

VIRGINIA TECH -- J Ficenec, D A Jenkins

Accelerator CEBAF Detector CLAS

Reactions Polarized beam

$$
\begin{array}{cc}
\gamma p \rightarrow \rho^{0} p & 1.0-1.5 \mathrm{GeV}\left(\mathrm{E}_{\mathrm{lab}}\right) \\
\gamma p \rightarrow \rho^{+} n & " \\
\gamma p \rightarrow \rho^{0} p & 1.4-2.1 \mathrm{GeV}\left(\mathrm{E}_{\mathrm{lab}}\right) \\
\gamma p \rightarrow \rho^{+} n & "
\end{array}
$$

Particles studued $N^{*}$ (unspec), $\Delta$ (unspec)

Brief description Studies the baryon resonance region between 1.66 and $2.22 \mathrm{GeV}$ center-of-mass energy in the $\rho N$ channel. Uses a linearly polarized tagged photon beam produced by coherent bremsstrahlung from a diamond crystal. Measures the beam asymmetry and extracts the spin density matrix elements from the angular distribution of the two-pion decay of the $\rho$ meson as a function of $s$ and $t$. This facilitates the search for baryon resonance contributions and provides information on diffractive, $t$ - and $u$-channel processes. Scheduled to run in

Fiall $\mathrm{B}$, for 11 days at $4 \mathrm{GeV}$, and 9 days at $6 \mathrm{GeV}$.

Related experiments CEBAF-93-033

E-mail contact cole@microl.cebaf.gov

\section{Other CEBAF Experiments}

Listed here are some other Jefferson Lab's (formerly CEBAF) experiments of interest to the particle physics community. Find more details about these projects online, in the SLAC's EXPERIMENTS database (sce p. 3), or contact the spokespersons. The regularly published Program Advisory Committee (PAC) reports often list all the approved experiments at the Lab. For the copies of these reports contact users@cebaf.gov, or check the Lab's main Web page (http://www.cebaf.gov)

CEBAF-89-004 (In preparation)

ELECTROMAGNETIC PRODUCTION OF HYPERONS By CLAS Collaboration

Spokesperson: Reinhard A. Schumacher [Carnegic Mellon U.]

E-mail contact reinhard@ernest.phys.cmu.edu

CEBAF-89-008 (In preparation)

INCLUSIVE SCATTERING FOR NUCLEI AT $x>1$ AND HIGH $Q^{2}$

Spokespersons: Bradley W. Filippone [Cal Tech], Donal B. Day [Virginia U.]

$\underline{\text { E-mail contact }}$ brad@erin.caltech.edu, dbd@virginia.edu

CEBAF-89-009 (In preparation)

INVESTIGATION OF THE SPIN DEPENDENCE OF THE $\Lambda N$ EFFECTIVE INTERACTION IN THE $P$ SHELL Spokespersons: Robert E. Chrien [Brookhaven\}, Ed Hungerford [Houston U.]

E-mail contact chrien@bnl.gov, hunger@uh.edu

CEBAF-89-012 (Taking data)

TWO-BODY PHOTODISINTEGRATION OF THE

DEUTERON AT FORWARD ANGLES AND PHOTON

ENERGIES BETWEEN 1.5 AND $4.0 \mathrm{GeV}$

Spokesperson: Roy J. Holt [Argonne]

E-mail contact holt@anlphy.phy.anl.gov

CEBAF-89-015 (In preparation)

STUDY OF COINCIDENCE REACTIONS IN THE DIP AND $\triangle$-RESONANCE REGIONS

Spokesperson: Hossain Baghaei [Virginia U.]

E-mail contact hossain@virginia.edu

CEBAF-89-0I7 (In preparation)

ELECTROEXCITATION OF THE $\triangle$ (1232) IN NUCLEI

Spokesperson: Richard Sealock [Virginia U.]

E-manl contact sealock@virginia.edu

CEBAF-89-033 (In preparation)

MEASUREMENT OF RECOIL POLARIZATION IN THE ${ }^{16} \mathrm{O}\left(\vec{e}, e^{\prime} \vec{p}\right)$ REACTION WITH 4 GeV ELECTRONS

Spokespersons: Sirish Nanda, J.Wallace Van Orden [CEBAF], Chia Cheh (George) Chang [Maryland U.], Charles Glashausser [Rutgers U.]

E-mail contact nanda@cebaf.gov, vanorden@cebaf.gov,

chang@enp.umd.edu, glashausser@ruthep.rutgers.edu 


\section{SUMMARIES OF CEBAF EXPERIMENTS}

CEBAF-89-037 (In preparation)

ELECTROPRODUCTION OF THE $\triangle\left(1232 P_{33}\right)$ RESO-

NANCE

By N* Collaboration

Spokespersons: Volker D. Burkert [CEBAF], Ralph C. Minehart [Virginia U.]

E-mail contact burkert@cebaf.gov, minehart@virginia.edu

CEBAF-89-042 (In preparation)

MEASUREMENT OF THE ELECTRON ASYMMETRY

IN THE $p\left(e, e^{\prime} p\right) \pi^{0}$ AND $p\left(e, e^{\prime} \pi^{+}\right) n$ IN THE MASS

REGION OF THE $\Delta(1232 P 33)$ FOR $Q^{2} \leq 2(\mathrm{GeV} / c)^{2}$

By $\mathrm{N}^{*}$ Collaboration

Spokespersons: Volker D. Burkert [CEBAF], Ralph C. Minehart [Virginia U.]

$\underline{\text { E-mail contact }}$ burkert@cebaf.gov, minehart@virginia.edu

CEBAF-89-045 (In preparation)

STUDY OF KAON PHOTOPRODUCTION ON DEUTERIUM

Spokesperson: Bernhard A. Mecking [CEBAF]

E-mail contact mecking@cebaf.gov

CEBAF-91-003 (In preparation)

A STUDY OF LONGITUDINAL CHARGED PION ELECTROPRODUCTION IN ${ }^{2} \mathrm{D},{ }^{3} \mathrm{He}, \mathrm{AND}^{4} \mathrm{He}$

Spokesperson: Harold E. Jackson [Argonne]

E-mail contact hal@anl.gov

CEBAF-91-004 (In preparation)

MEASUREMENT OF STRANGE QUARK EFFECTS USING PARITY VIOLATING ELASTIC SCATTERING FROM ${ }^{4} \mathrm{He}$ AT $Q^{2}=0.6(\mathrm{GeV} / \mathrm{c})^{2}$

Spokesperson: Betsy Beise [Maryland U.]

E-mail contact beise@enp.umd.edu

CEBAF-91-007 (In preparation)

MEASUREMENT OF THE NUCLEAR DEPENDENCE AND MOMENTUM TRANSFER DEPENDENCE OF QUASIELASTIC $\left(e, e^{\prime} p\right)$ SCATTERING AT LARGE MOMENTUM TRANSFER

Spokesperson: Richard G. Milner [MIT]

E-mail contact milner@mitlns.mit.edu

CEBAF-91-010 (In preparation)

PARITY VIOLATION IN ELASTIC SCATTERING FROM THE PROTON AND ${ }^{4} \mathrm{He}$

Spokespersons: Paul A. Souder [Syracusc U.], J.M. Finn [William and Mary Coll.]

E-mail contact souder@suhep.phy.syr.edu, finn@cebaf.gov WWW Home-page http://www.cebaf.gov/halla/parity.html

CEBAF-9t-014 (In preparation)

QUASIFREE STRANGENESS PRODUCTION IN NUCLEI

Spokesperson: Charles E. Hyde-Wright [Old Dominion U.]

E-mail contact hyde@cebaf.gov
CEBAF-91-015 (In preparation)

HELICITY STRUCTURE OF PION PHOTOPRODUCTION

By CLAS Collaboration

Spokesperson: Daniel I. Sober [Catholic U.]

E-mail contact sober@cua.edu

CEBAF-91-016 (In preparation)

ELECTROPRODUCTION OF KAONS AND LIGHT HYPERNUCLEI

Spokesperson: Benjamin Zeidman [Argonne]

E-mail contact zeidman@anlphy.phy.anl.gov

CEBAF-91-017 (In preparation)

MEASUREMENT OF $G^{0}$, THE FLAVOR SINGLET CHARGE FORM-FACTOR OF PROTON

Spokesperson: Douglas H. Beck [Illinois U., Urbana]

E-mail contact beck@uinpla.npl.uiuc.edu

CEBAF-91-026 (In preparation)

MEASUREMENT OF THE ELECTRIC AND MAGNETIC STRUCTURE FUNCTIONS OF DEUTERON AT LARGE MOMENTUM TRANSFERS

Spokesperson: Gerassimos (Makis) G. Petratos [Kent State U.]

E-mail contact petratos@ksuvxd.kcnt.edu

CEBAF-93-008 (In preparation)

INCLUSIVE $\eta$ PHOTOPRODUCTION IN NUCLEI

Spokesperson: Michael F. Vineyard [Richmond U.]

E-mail contact vineyard@urvax.urich.edu

CEBAF-93-009 (In preparation)

THE POLARIZED STRUCTURE FUNCTION $G_{1 n}$ AND THE $Q^{2}$ DEPENDENCE OF THE GERASIMOV-DRELLHEARN SUM RULE FOR THE NEUTRON

By $N^{*}$ Collaboration

Spokesperson: Sebastian E. Kuhn [Old Dominion U.]

E-mail contact kuhn@cebaf.gov

CEBAF-93-012 (in preparation)

ELECTROPRODUCTION OF LIGHT QUARK MESONS By CLAS Collaboration

Spokesperson: Mikhail V. Kossov [Moscow, ITEP]

E-mail contact kossov@cebaf.gov

CEBAF-93-017 (In preparation)

STUDY OF $\gamma d \rightarrow p n$ AND $\gamma d \rightarrow p \Delta^{0}$ REACTIONS FOR SMALL MOMENTUM TRANSFERS

Spokespersons: Enzo De Sanctis, Patrizia Rossi [Frascati]

E-mail contact rossi@lnf.infn.it, desanctis@lnf.infn.it

CEBAF-93-021 (In preparation)

THE CHARGED PION FORM-FACTOR

Spokesperson: David J. Mack [CEBAF]

E-mail contact mack@cebaf.gov 


\section{SUMMARIES OF CEBAF EXPERIMENTS}

CEBAF-93-022 (In preparation)

MEASUREMENT OF THE POLARIZATION OF THE $\phi$ IN ELECTROPRODUCTION

Spokespersons: Elton Smith [CEBAF], Philip D. Rubin [Richmond U.], Herbert O. Funsten [William and Mary Coll.]

$\underline{E-m a i l}$ contact elton@cebaf.gov, rubin@bart.urich.edu, funsten@cebaf.gov

CEBAF-93-024 (In preparation)

MEASUREMENT OF $T H E$ MAGNETIC FORM-FACTOR OF THE NEUTRON AT LARGE MOMENTUM TRANSFERS

Spokespersons: Javier Gomez [CEBAF], Gerassi-

mos (Makis) G. Petratos [Kent State U.]

E-mail contact gomez@cebaf.gov, gpetrato@kentvm.kent.edu

\section{CEBAF-93-026 (In preparation)}

THE CHARGE FORM-FACTOR OF THE NEUTRON

Spokesperson: Donal B. Day [Virginia U.]

E-mail contact dbd@virginia.edu

CEBAF-93-027 (In proparation)

ELECTRIC FORM-FACTOR OF PROTON BY RECOIY POLARIZATION

Spokespersons: Vina Punjabi [Norfolk State U.]. Charles F. Per drisat [William and Mary Coll.]

E-mail contact punjabi@cebaf.gov, perdrisat@cebaf.gov

\section{CEBAF-93-031 (In preparation)}

\section{PHOTOPRODUCTION OF VECTOR MESONS AT}

HIGH $t$

Spokespersons: Marco Anghinolfi [INFN, Genoa],

Jean Marc Laget, Claude Marchand [Saclay]

$\underline{\text { E-mail contact }}$ anghi@genova.infn.it, laget@phnx7.saclay.cea.fr, marchand@phnx7.saclay.cea.fr

CEBAF-93-038 (In preparation)

THE ELECTRIC AND MAGNETIC FORM-FACTORS OF THE NEUTRON FROM THE $d\left(\vec{e}, e^{\prime} \vec{n}\right) p$ REACTION

Spokesperson: Richard Madey [Hampton U.]

E-mail contact madey@cebaf.gov

CEBAF-94-002 (In preparation)

PHOTOPRODUCTION OF VECTOR MESONS OFF
NUCLEI

Spokespersons: Pierre Bertin [Clermont-Ferrand U.],

Mikhail Vladimirovich Kossov [Moscow, ITEP], Barry Preedom [South Carolina U.]

E-mail contact kossov@cebaf.gov

CEBAF-94-005 (In preparation)

DETERMINATION OF THE $N \triangle$ AXIAL VECTOR TRANSITION FORM-FACTOR $G_{N \Delta}^{A}$ FROM THE $e p \rightarrow e^{\prime} \Delta^{++} \pi^{-}$REACTION

Spokespersons: Latifa Elouadrhiri, David Heddle [Christopher Newport U. ]

E-mail contact latifa@cebaf.gov
CEBAF-94-010 (In preparation)

MEASUREMENT OF THE NEUTRON $\left({ }^{3} \mathrm{He}\right)$ SPIN STRUCTURE FUNCTION AT LOW $Q^{2}$ : A CONNECTION BETWEEN THE BJORKEN AND DRELLHEARN-GERASIMOV SUM RULES

Spokespersons: Gordon D. Cates [Princeton U.], ZeinEddine Meziani ['Temple U.]

E-mail contact meziani@vm.temple.edu

CEBAF-94-012 (In preparation)

MEASUREMENT OF PHOTOPROTON POLARIZA

TION IN THE $\mathrm{H}(\gamma, \vec{p}) \pi^{0}$ REACTION

Spokespersons: Roy J. Holt [Illinois U., Urbana], Ronald Gilman [Rutgers U.]

$\underline{E-m a i l}$ contact gilman@ruthep.rutgers.edu

CEBAF-94-014 (In preparation)

The $\triangle(1232)$ FORM-FACTOR AT HIGH MOMENTUM TRANSFER

Spokesperson: James 3. Napolitano [Rensselaer Poly]

E-mail contact jimnap@rpi.edu

CEBAF-94-015 (In preparation)

STUDY OF THE AXIAL ANOMALY USING THE $\gamma \pi^{+} \rightarrow$ $\pi^{+} \pi^{0}$ REACTION NEAR THRESHOLD

Spokespersons: Amrit S. Yegneswaran [CEBAF], Rory A. Miskimen [Massachusetts U., Amberst], Kebin Wang [Virginia U.]

E-mail contact miskimen@phast.umass.edu

CEBAF-94-017 (In proparation)

THE NEUTRON MAGNETIC FORM-FACTOR FROM

PRECISION MEASUREMENTS OF THE RATIO

OF QUASIELASTIC ELECTRON-NEUTRON TO

ELECTRON-PROTON SCATTERING IN DEUTERIUM

Spokespersons: William K. Brooks [CEBAF], Michael F. Vincyard [Richmond U.]

E-mail contact brooksw@cebaf.gov

CEBAF-94-103 (In preparation)

THE PHOTOPRODUCTYON OF PIONS

Spokespersons: William J. Briscoe [George Washington U.], John Ficenec, David A. Jenkins [Virginia Tech]

E-mail contact jficenec@vt.edu

CEBAF-94-104 (In preparation)

THE FUNDAMENTAL $\gamma n \rightarrow \pi-p$ PROCESS IN ${ }^{2} \mathrm{H},{ }^{4} \mathrm{He}$, and ${ }^{12} \mathrm{C}$ IN THE 1.2-6.0 GeV REGION

Spokespersons: Haiyan Gao, Roy J. Holt [Illinois U., Urbana]

E-mail contact gao@uinpla.npl.uiuc.edu

CEBAF-95-003 (In preparation)

MEASUREMENT OF $K^{0}$ ELECTROPRODUCTION

Spokesperson: Richard Magahiz [Carnegie Mellon U.]

E-mail contact magahiz@ernest.phys.cmu.edu 


\section{SUMMARIES OF CERN EXPERIMENTS}

\section{Selected CERN Experiments}

\section{CERN-IS-300}

(Proposed Sep 1991, Approved Sep 1991, In preparation)

\section{A SEARCH FOR AXIONS AND MASSIVE NEUTRINOS}

IS300-ISOLDE COLLABORATYON

AARHUS U - P Flornshoj, H L Nicisen, J W Petersen, K Riisager, G Weyer ( $\checkmark$ Spokesperson)

CERN - A De Rujula, H L Ravn

CHALMERS UNIV TECH - B Jonson, G Nyman

Accelerator CERN-PS Detector Spectrometer

Particles studied axion, $\nu$

Brief description A search for axions and a heavy neutrino

relying on a strong, high purity source of radioactive ${ }^{125} \mathrm{I}$. A possible $17 \mathrm{keV}$ neutrino emission in the clectron capture decay of ${ }^{125}$ I could be observed by studying the internal bremsstrahlung spectrum. A possible observation of axions in the $35.5 \mathrm{keV}$ M1 transition of the ${ }^{225} \mathrm{Te}$ daughter is based on an analogue of the Mössbauer effect, i.e. the axion resonance absorption in the 125 , re resonance absorber. Awaiting for a staggered beam from the PS Booster, expected to be available in Spring 97.

E-mailcontact helge.ravn@cern.ch

\section{CERN-LEP-ALEPH}

(Proposed 1982, Approved Nov 1982, Began data-taking Aug 1989, In progress)

\section{THE ALEPH DETECTOR (APPARATUS FOR $L E P$} PHYSICS)

\section{ALEPH COLLABORATION}

ANNECY - D Buskulic, I De Bonis, D Decamp, P Ghez, C Goy, I P Lees, A lucotte, M N Minard, P Odier, B Pietrzyk

BARCELONA, AUTONOMA U - M Chmeissani, J M Crespo, $M$ Delfino, I Efthymiopoulos, M Fernandez-Bosman,

E Fernandez, L Garrido, A Juste, M Martinez, S Orteu, A Pacheco, C Padilla, F Palla, A Pascual, J A Perias, I Riu, F Sanchez, F Teubert

BARI U \& INFN, BARI - A Colaleo, D Creanza, M de Palma, A Farilla, G Gelao, M Girone, G Iasetli, G Maggi, M Maggi, N Marinelli, S Natali, S Nuzzo, A Ranieri, G Raso, F Romano, F Ruggieri, G Selvaggi, L Silvestris, P Tempesta, G Zito

BEIJING, IHEP - X Huang, J Lin, Q Ouyang, T Wang, $X X i e$, $R$ Xu, S Xue, J Zhang, L Zhang, W Zhao

CERN - R Alemany, A O Bazarko, G Bonvicini, M Cattaneo, P Comas, P Coyle, H Drevermann, R W Forty, M Frank, R Hagelberg, J Harvey, R Jacobsen, P Janot, B Jost, E Kneringer, J Knobloch, X Lehraus, E B Martin, P Mato, A Minten, R Miquel, L M Mir, L Moneta, 'T Oest. P Palazzi, I R Pater, J F Pusztaszeri, F Ranjard, P Rensing, L Rolandi ( $\sqrt{ }$ Spokesperson), D Schlatter, M Schmelling, O Schneider, W Tejessy, I R Tomalin, A Venturi, H Wachsmuth, A Wagner. T Wildish, W Witzeling, J Wotschack

CLERMONT-FERRAND U - Z Ajaltouni, A Barres, C Boyer,

A Falvard, P Gay, C Guicheney, P Henrard, J Jousset, B Michel, S Monteil, J C Montret, D Pallin, P Perret, F Podlyski, J Proriol, J M Rossignol

BOHR INST - T Fearnley, J B Hansen, J D Hansen, J R Hansen, P H Hansen, B S Nilsson, A Waananen

DEMOCRITOS NUCLEAR RESEARCH CENTER - A Kyriakis,

C Markou, E Simoponlou, I Siotis, A Vayaki, K Zachariadou

ECOLE POLYTECHNIQUE - A Blondel. G Bonneaud,

P Bourdon, J C Brient, A Rouge, M Rumpf, R Tanaka A Valassi, M Verderi, H Videau

EDINBURGH U - D J Candlin, M I Parsons

FLORENCE U \& INFN, FLORENCE - E Focardi, G Parrini FLORIDA STATE U -- M Corden, C Georgiopoulos, D E Jaffe FrASCATX ... A Antonelli, G Bencivenni, G Bologna, F Bossi,

P Campana, G Capon, D Casper, V Chiarella, G Felici,
P Laurelli, G Mannocchi, F Murtas, G P Murtas, L Passalacqua, M Pepe-Altarelli

GLASGOW U - L Curtis, S J Dorris, A W Halley, I G Knowles,

J G Lynch, V O'Shea, C Raine, P Reeves, J M Scarr, K Smith, A S Thompson, $F$ 'Thomson, $S$ Thorn, $R$ M Turnbull

HEIDELBERG U - U Becker, C Geweniger, G Graefe, $P$ Hanke,

V Hepp, E E Kluge, A Putzer, B Rensch, M Schmidt,

J Sommer, H Stenzel, K Tittel, S Werner, M Wunsch

IMPERIAL COLL - D Abbaneo, $R$ Beuselinck, D M Binnie,

W Cameron, P J Dornan, A Moutoussi, J Nash, G San Martin, J K Sedgbeer, A M Stacey, M D Williams

INNSBRUCK U - G Dissertori, P Girtler, D Kuhn, G Rudolph

LANCASTER U - C K Bowdery, T J Brodbeck, P Colrain,

G Crawford, A J Finch, F Foster, G Hughes, T Sloan

E P Whelan, M I Williams

MAINZ U, INST PHYS - A Galla, A M Greene, K Kleinknecht,

G Quast, B Renk, E Rohne, H G Sander, P van Gemmeren,

R Wanke, C Zcitnitz

MARSEILLE, CPPM - J J Aubert, A M Bencheikh, C Benchouk, A Bonissent, G Bujosa, D Calvet, J Carr, C Diaconu, F Etienne, N Konstantinidis, D Nicod, P Payre, D Roussean, A Sadouki, M Talby, M Thulasidas, $K$ Trabelsi

MUNICH, MAX PLANCK INST - I Abt, R Assmann, C Bauer,

W Blum, H Dietl, F Dydak, G Ganis, C Gotzhein, K Jakobs,

I Kroha, G Lutjens, G Lutz, W Manner, H G Moser, R Richter, $\Lambda$ Rosado-Schlosser, S Schael, R Settles, H Seywerd, R St Denis, W Wiedenmann, $G$ Wolf

ORSAY, LAL - J Boucrot, O Callot, A Cordicr, M Davier, L Duflot, J F Grivaz, P Heusse, M Jacquet, D W Kim, F Le Diberder, J Lefrancois, A M Lutz, I Nikolic, H J Park, I C Park, M H Schune, S Simion, J J Veillet, I Videau

PISA U \& INFN, PISA \& PISA, SCUOLA NORMALE SUPERIORE - P Azzurri, G Bagliesi, G Batignani, S Bettarini, C Bozzi, G Calderini, M Carpinelli, M A Ciocci, V Ciulli, R Dell'Orso, R Fantechi, I Ferrante, L Foa, F Forti, A Giassi, M A Giorgi, A Gregorio, F Ligabue, A Lusiani, P S Marrocchesi, A Messineo, G Rizzo, G Sanguinetti, A Sciaba, P Spagnolo, J Steinberger, R Tenchini. G Tonelli, C Vannini, P G Verdini, $\mathrm{J}$ Walsh

ROYAL HOLLOWAY - BEDFORD COLL - A P Betteridge, G A Blair, L M Bryant, F Cerutti, J 'T' Chambers, Y Gao, M G Green, D L Johnson, T Medcalf, P Perrodo, J A Strong, $3 \mathrm{H}$ von Wimmersperg-Toller

RUTHERFORD - D R Botterill. R W Clifft, T R Edgecock, $S$ Haywood, P Maley, P R Norton, J C Thompson

DAPNIA, SACLAY - B Bloch-Devaux, P Colas. S Emery,

W Kozanecki, E Lancon, M C Lemaire, E Locci, B Marx, P Perez, J Rander, J F Renardy, A Roussaric, J P Schuller, I Schwindling, A Trabelsi, B Vallage

UC, SANTA CRUZ - R P Johnson, H Y Kim, A M Litkc, M A McNeil, G Taylor

SHEFFIELD U - A Beddall, C N Booth, R Boswell, C A J Brcw, S Cartwright, F Combley, A Koksal, M Letho, W M Newton, C Rankin, J Reeve, L $F$ Thompson

SIEGEN U - $A$ Bohrer, S Brandt, V Buscher, G Cowan, E Feigl, C Grupen, G Lutters, J Minguet-Rodriguez, F Rivera, P Saraiva, L Smolik, F Stephan

TRIESTE U, IST FIS \& INFN, TRIESTE - M Aleppo,

N Apollonio, L Bosisio, R Della Marina, G Giannini, B Gobbo, G Musolino, $F$ Ragusa

WASHINGTON U, SEATTLE - I Rothberg, S Wasserbaech

WISCONSIN U - S R Armstrong, L Bellantoni, P Elmer, Z Feng, D P S Ferguson, Y S Gao, S Gonzales, J Grahl, 'T C Grecning, I L Harton, O J Hayes, H I u, P A McNamara, III , J M Nachtman, W Orejudos, Y B Pan, Y Saadi, M Schmitt, I J Scott, V Sharma, J D Turk, A M Walsh, S L Wu, X Wu, J M Yamartino, M Zheng, G Zobernig

Accelerator CERN-LEP Detector ALEPH

Reactions

$e^{+} e^{-} \quad<200 \mathrm{GeV}\left(\mathrm{E}_{\mathrm{cm}}\right)$

Partzcles studied $W^{+}, W^{-}, Z^{0}$, hvy-flavor, hvy-lepton, higgs, s-particle

Brief description A $4 \pi$ detector designed to give as much detailed information as possible about complex events in highencrgy $\mathrm{e}^{+} e^{-}$collisions. $\Lambda$ superconducting coil $5 \mathrm{~m}$ in diameter and $6 \mathrm{~m}$ long produces a uniform 1.5 tesla field in the beam direction. Inside the coil, in order of increasing radius, there 


\section{SUMMARIES OF CERN EXPERIMENTS}

is a microstrip solid state device, an Inner Tracking Chamber (ITC) using drift wires, a Time Projection Chamber (TPC), $3.6 \mathrm{~m}$ diameter, $4.4 \mathrm{~m}$ long, and an electromagnetic calorimeter, of $2 \mathrm{~mm}$ lead sheets with proportional wire sampling. Outside of the coil, a $1.2 \mathrm{~m}$ thick Fe return path is used as a hadron calorimeter, and a double layer of drift tubes aids in the muon identification. Strong points of the detector are a precision of momentum measurements for charged particles, due to a high magnetic field and a TPC, a good identification of electrons and muons even when they are immersed in jets, and a spatial resolution obtained in $e \gamma$ calorimetry. A minivertex detector provides a capability for identifying secondary vertices, and a silicon-tungsten calorimeter installed in 1992 allows a significant reduction of the luminosity error. Taking data (July 96).

Journal papers NIM 217 (1983) 305, NIM 217 (1983) 317, NIM A225 (1984) 481, NIM A226 (1984) 82, IEEE TNS 32 (1985) 605, NIM A228 (1985) 327, NIM A234 (1985) 47, NIM A235 (1985) 296, NIM A239 (1985) 192, NIM A244 (1986) 516, NIM A247 (1986) 438, NIM A251 (1986) 449, NIM A252 (1986) 392, NLM A252 (1986) 399, NIM A252 (1986) 403, IEEE TNS 34 (1987) 133, CPC 45 (1987) 229, CPC 45 (1987) 283, CPC 45 (1987) 433, NIM A257 (1987) 587, IEEE TNS 35 (1988) 316, NIM A263 (1988) 43, NIM A263 (1988) 58, NIM A268 (1988) 144, NIM A271 (1988) 449, CPC 57 (1989) 401, IEEE TNS 36 (1989) 1459, IEEE TNS 36 (1989) 3464, IEEE TNS 36 (1989) 1514, NIM A277 (1989) 358, NIM A279 (1989) 212, NIM A283 (1989) 573, PL B231 (1989) 519, IJMP Cl (1990) 147, IEEE TNS 37 (1990) 1210, NIM 4286 (1990) 61, NIM $A 289$ (1990) 176, NIM A294 (1990) 121 [erratum: NIM A303 (1991) 393], NIM A297 (1990) 153, NIM A297 (1990) 390, HEPNP 14 (1990) 966, PL B234 (1990) 209, PL B234 (1990) 399, PL B235 (1990) 399, PL B236 (1990) 86, PL B236 (1990) 233, PL B236 (1990) 501, PL B236 (1990) 511, PL B237 (1990) 291, PL B241 (1990) 141, PL B241 (1990) 623, PL B241 (1990) 635, PL B244 (1990) 541 , PL B244 (1990) 551, PL B245 (1990) 289, PL B246 (1990) 306, PL B250 (1990) 172, ZPHY C48 (1990) 365, NIM A306 (1991) 446, NP (PROC SUPPL) 23B (1991) 291, PL B255 (1991) 623, PL B257 (1991) 479, PL B257 (1991) 492, PL B258 (1991) 236, PL B259 (1991) 377, PL B262 (1991) 139. PL B263 (1991) 112, PL B263 (1991) 325, PL B264 (1991) 476, PL B265 (1991) 430, PL B265 (1991) 475, PL B266 (1991) 218, PL B273 (1991) 181, NIM A315 (1992) 121, NIM A320 (1992) 177, NIM A323 (1992) 213, PL B276 (1992) 247. PL B278 (1992) 209, PL B279 (1992) 411, PL B284 (1992) 151, PL B284 (1992) 163, PL B284 (1992) 177, PL B285 (1992) 309, PL B292 (1992) 210, PL B294 (1992) 145, PL B295 (1992) 174, PL B295 (1992) 396, PL B297 (1992) 432, PL B297 (1992) 449, PL B297 (1992) 459, PRPL 216 (1992) 253, ZPHY C53 (1992) 1, ZPHY C53 (1992) 21, ZPHY C53 (1992) 375, ZPHY C54 (1992) 75, ZPHY C54 (1992) 211, ZPHY C55 (1992) 209, PL B298 (1993) 479, PL B303 (1993) 198, PL B307 (1993) 187, PL B307 (1993) 194, PL B307 (1993) 209, PL B308 (1993) 425, PL B311 (1993) 425 [erratum: PL B316 (1993) 631], PL B313 (1993) 299, PL B313 (1993) 312, PL B313 (1993) 498, PL B313 (1993) 509, PL B313 (1993) 520, PL B313 (1993) 535, PL B313 (1993) 549, PL B314 (1993) 459, ZPHY C57 (1993) 17, ZPHY C59 (1993) 215, ZPHY C59 (1993) 369, ZPHY C60 (1993) 71, IEEE TNS 41 (1994) 236 , NIM A346 (1994) 461, PL B321 (1994) 168, PL B322 (1994) 275, PL B322 (1994) 441, PL B332 (1994) 209, PL B332 (1994) 219, PL B334 (1994) 244, PL B335 (1994) 99, ZPHY C62 (1994) 1, ZPHY C62 (1994) 179, ZPIIY C62 (1994) 539, ZPHY C64 (1994) 361, NIM A360 (1995) 481, NP (PROC SUPPL) 39BC (1995) 322, PL B343 (1995) 444, PL B345 (1995) 103, PL B346 (1995) 371, PL B346 (1995) 379, PL B346 (1995) 389, PL B349 (1995) 238, PL B349 (1995) 585, PL B352 (1995) 479, PL B355 (1995) 381, PL B355 (1995) 595, PL B356 (1995) 409, PL B357 (1995) 487 [erratum: PL B364 (1995) 247], PL B357 (1995) 685, PL B357 (1995) 699, PL B359 (1995) 236, PL B361 (1995) 221, ZPHY C66 (1995) 3, ZPHY C66 (1995) 355, ZPHY C69 (1995) 15, PL B365 (1996) 437, PL B369 (1996) 151, PL B373 (1996) 246, PL B374 (1996) 319, ZPHY C69 (1996) 183, ZPHY C69 (1996) 365, ZPHY C69 (1996) 379, ZPHY C69 (1996) 393, and 2PHY C69 (1996) 585.

\section{E-mail contact luigi.rolandi@cern.ch}

WWW Home-page http://alephwww.cern.ch/WWW/

\section{CERN-LEP-DELPHI}

(Proposed 1982, Approved Nov 1982, Began data-taking Aug 1989, In progress)

\section{THE DELPHI DETECTOR (DETECTOR WITH} $L E P T O N$ PHOTON AND HADRON IDENTIFICATION)

\section{DELPHI COLLABORATION}

ANTWERP U \& BRUSSELS U, ILHE \& MONS U - D Bertrand, C Bricman, F Cao, $M$ Chen, A Deghorain, S De Brabandere, $C$ De Clercq, $P$ Herquet, $S$ Hoorelbeke, $K$ Huet, $V$ Lefebure, $J$ Lemonne, A Tomaradzc, C Vander Velde, W K Van Doninck, $\mathrm{F}$ Verbeure, $3 \mathrm{H}$ Wickens

IOWA STATE U -- H B Crawley, D Edsall, A Firestone, L Gorn, T S Hill, J W Lamsa, D W Lane, C K Legan, R Mc Kay, W T Meyer, E I Rosenberg

ATHENS U - S Katsanevas, C Kourkoumelis, R Nicolaidou, L K Resvanis

BERGEN U - G Eigen, A G Frodesen, R Keranen, A Klovning, B Stugu

BOLOGNA U \& INFN, BOLOGNA - A C Benvenuti,

F R Cavallo, F L Navarria, A Perrotta, T Rovelli, G Valenti

RIO DE JANEIRO, CBPF \& RIO DE JANEIRO, PONT UNIV CATOLICA \& RIO DE JANEIRO STATE U - M Begalli,

M Gandelman, L M Mundim, M E Pol, R C Shellard, D SouzaSantos

COMENIUS U - P Chochula, R Janik, P Kubinec, B Sitar

COLLEGE DE FRANCE - P Beilliere, J M Brunet, C Defoix, J Dolbeau, P Frenkiel, G Tristram

CERN - U Amaldi, P Antilogus, A Augustinus, P Baillon,

Y Belokopytov, C Bourdarios, R C A Brown, A Buys,

T Camporesi, F Carena, A Cattai, V Chabaud, P Charpentier. V Chorowicz, P Collins, M Davenport, A De Angelis, H Dijkstra, M Donszelmann, M Feindt, H Foeth, F Formenti, C Gaspar, P Gavillet, F Hahn, H Herr, H J Hilke, C Joram,

K Klein, M Koratzinos, J C Marin, M Mc Cubbin,

M Paganoni, L Pape, D Reid, E Rosso, F Stichelbaut, $D$ Treille ( $\checkmark$ Spokesperson $)$, W Trischuk, A Tsirou, O Ullaland, E Vallazza, P Weilhammer, A M Wetherell, A Zalewska

STRASBOURG, CRN - D Bloch, F Djama, M Dracos, J P Engel, P A Fischer, D Gele, 3 P Gerber, P $y_{\text {uillot, }} V$ Nikolaenko, $P$ Pages, R Strub, 'T' Todorov, S Todorova, M Winter

DEMOCRITOS NUCLEAR RESEARCH CENTER -

K Karafasoulis, E Karvelas, P Kokkinias, D Loukas, A Markou. K Papageorgiou, E Zevgolatakos

PRAGUE, INST PIYS - S Nemecek, M Novak, J Rames,

J Ridky, V Vrba

GENOA U \& INFN, GENOA - M Bozzo, M Canepa, C Caso, R Contri, G Crosetti, F Fontanelli, V Gracco, O Kouznetsov, M R Monge, P Morettini, F Parodi, A Petrolini, G Piana,

I Roncagliolo, M Samnino, S Squarcia

GRENOBLE U .. M L Andrieux, $R$ Barate, F Ledroit, F Naraghi. L Roos, O Sahr, G Sajot

HELSINKI U - M Battaglia, R A Brenner, S Czellar, K Kurvinen, R Lauhakangas, R Orava, $K$ Osterberg, H Saarikko

DUBNA - G D Alekseev, D X Bardin, M S Bilenky, G A Chelkov, B A Khomenko, N N Khovanski, Z Krumstein, V Malychev, A G Olshevski, V Pozdniakov, N Pukhacva, A Sadovsky, Y Sedykh, A N Sisakian, L G Tkatchev, I A Tyapkin, L S Vertogradov, A S Vodopyanov. N I Zimin

KARLSRUHE U -. W D Apel, W De Boer, R Ehret, D C Fries, M Kaiser, C Kreuter, G Maehlum, S Meyer, $H$ Mueller, W Oberschulte-Beckmann, O Podobrin, M Schimmelpfennig. H Schneider, U Schwickerath, A Seitz, C Weiser, M Wielers

CRACOW - P Bruckman, Z Hajduk, P Jalocha, K Korcyl,

W Krupinski, W Kuccwicz, T Lesiak, B Muryn, H Palka, G Polok, K Rybicki, M Witek

ORSAY, LAL - P Bambade, B Bouquet, J L Contreras, G Cosme, B Dalmagne, F Fulda-Quenzer, G Grosdidier, B Jean-Marie, V Lepeltier, P Paganini, S Plaszczynski, P Rebecchi, F Richard, P Roudeau, A Stocchi, A Trombini

LANCASTER U .. P N Ratoff, P Seager

LISBON, LIP - P Abreu, F Barao, M Espirito Santo,

$R$ Henriques, A Majo, $A$ Onofre, L Peralta, M Pimenta, T Spassov, B Tome

LIVERPOOL U - P P Allport, P S L Booth, T J V Bowcock, L Carroll, J H Cowell, A Galloni, M Gibbs, C Green,

M Houlden, J N Jackson, B King, S Marti i Garcia, R Mc Nulty, J Richardson, S Tzamarias 


\section{SUMMARIES OF CERN EXPERIMENTS}

PARIS, CURIE UNIV VI - M Baubillier, P Billoir, W Da Silva. $C$ De La Vaissiere, S Fichet, F Kapusta, R Pain, J P Tavernet LUND U - S Almehed, O Barring, E Falk, V Hedberg, C Jariskog, $G$ Jarlskog, L Jonsson, P Jonsson, I Kronkvist, B Loerstad, U Mjoernmark, O Smirnova, G Transtromer

LYON, IPN - J E Augustin, D Bertini, L Chaussard, J D Durand, I Laktineh, L Mirabito, G Smadja, P Vincent, F Zach

MADRID U - J A Barrio, J Sanchez

MARSEILLE, CPPM - P Delpierre, A Tilquin

MILAN U \& INFN, MILAN - A Andreazza, M Bonesini, W Bonivento, M Caccia, M Calvi, X Gouz, S Gumenyuk, C Matteuzzi, C Meroni, P Negri, A Pullia, S Ragazzi, N G Redaelli, T Tabarelli, A Tonazzo, C Troncon, G Vegni BOHR INST - E Dahl-Jensen, M Dam, G Damgaard, N J Kjaer, $R$ Moeller, B S Nielsen

CHARLES U - R Leitner

NIKHEF, AMSTERDAM - E Agasi, E Boudinov, W Hao,

D Holthuizen, P Kluit, B Kocne, M Merk, M Nieuwenhuizen, W Ruckstuhi, I Siccama, J Timmermans, D Z Toet, G W Van Apeldoorn, P Van Dam, J Van Eldik

DEMOCRITOS NUCLEAR RESEARCH CENTER -

M Dris, D Fassouliotis, T A Filippas, E Fokitis, E N Gazis, E C Katsoufis, T D Papadopoulou, H Rahmani

OSLO U - I Bugge, T Buran, A L Read, T B Skaali, S Stapnes OVIEDO U - J Cuevas Maestro

OXFORD U - N Demaria, F J Harris, T L Hessing, P J Holt, J Libby, J G Loken, L Lyons, G Myatt, A Normand, C Parkes, D Radojicic, P B Renton, A M Segar, K Stevenson, J Thomas, N Vassilopoulos, G R Wilkinson, W S C Williams, K Yip

PADUA U \& INFN, PADUA - K D Brand, P Checchia, A De Min, U Gasparini, A Lipniacka, I Lippi, M Margoni, M Mazzucato, M Michclotto, A Nomerotski, M Pegoraro, P Ronchese, F Simonetto, I Stavitski, L Ventura, M Verlato, $\mathrm{G}$ Zumerle

RUTHERFORD - I Adye, M J Bates, D Crennell, P D Dauncey, B Franek, G Gopal, J Guy, W J Murray, H T Phillips, R Sekulin, G R Smith, M 'Tyndel, W Venus ( $\checkmark$ Deputy Spokesperson)

ROME U, 'IORVERGATA \& INFN, ROME - V Canale, L Di Ciaccio, G Matthiae, P Privitera, L Serbelloni

DAPNIA, SACLAX -- J Baudot, M Besancon, T Bolognese, $G$ Borisov, C De Saint-Jcan, P Gris, P Jarry, J P Laugier, P Lutz, A Ouraou, F Pierre, I Ripp, V Ruhlmann-Kleider, $X$ Sacquin, P Sicgrist, $R$ Silvestre, S Simonetti, M L Turluer, D Vilanova, $M$ Zito

ROME, ISS \& INEN, ROME - A Baroncelli, C Bosio, P Branchini, E Graziani, C Mariotti, A Passeri, E Spiriti, C Stanescu, L Tortora

SANTANDER U - A J Camacho Rozas, J Garcia, J M Lopez, J Marco, R Marco, C Martinez-Rivero, F Matorras, A Ruiz SERPUKHOV - I Ajinenko, M Chapkin, P Chliapnikov, A Fenyuk, V Kostioukhine, V Lapin, V Obraztsov, A Ostankov, M Petrovyck, N Smirnov, O Tchikilev, V Uvarov, E Vlasov, A Zaitsev

STEFAN INST, LJUBEJANA \& LJUBLJANA U - V Cindro, B Erzen, B Golob, D Zavrtanik, D Zontar

STOCKHOLM U - B Asman, K Cankocak, G Ekspong, $\mathrm{P}$ Gunnarsson, S O Holmgren, $\mathrm{K}$ Hultqvist, $\mathrm{R}$ Jacobsson, E K Johansson, M Karlsson, 'T G M Malmgren, T Moa, P Niss, C Walck, G C Zucchelli

TURIN U \& INFN, TURIN - F Bianchi, M Bigi, R Chierici, D Gamba, E Migliore, G Rinaudo, A Romero, G Sciolla

TRIESTE U, IST FIS \& INFN, TRIESTE \& UDINE U G Barbiellini, F Cossutti, G Della Ricca, B De Lotto, L Lanceri, C Petridou, P Poropat, F Scuri, L Vitale, F Waldner

RIO DE JANEIRO U - $S$ Amato, M Barbi, M Berggren, L De Paula, B Marechal

UPPSALA U - O Botner, T Ekelof, M Gunther, A Hallgren, $\mathrm{J}$ Medbo, $\mathrm{K}$ Woschnagg

VALENCIA U - R Alemany, S Cabrera, M V Castillo Gimenez, E Cortina, A Ferrer, J Fuster, C Garcia, J J Hernandez, E Higon, C Lacasta, F Martinez-Vidal, S Navas, J Salt

VIENNA, OAW - W Adam, W Bart1, R Fruhwirth, J Hrubec, M Krammer, G Leder, D Liko, J MacNaughton, F Mandl, W A Mitaroff, N Neumeister, H Pernegger, M Pernicka, M Regler, J Strauss

WARSAW, INR - K Doroba, R Gokieli, M Gorski, K Grzelak, K Nawrocki, R Sosnowski, M Szczekowski, M Szeptycka, P Zalewski
WUPPERTAL U - K H Becks, M Blume, T Brenke, T Burgsmueller, P Buschmann, J Dahm, J Drees, $K$ A Drees, $M$ Elsing, A Grefrath, S Hahn, $\mathrm{K}$ Hamacher, O Klapp, P Langefeld, G Lenzen, $R$ Lindner, $K$ Moenig, W Neumann, M Reale, M A E Schyns, P Sponholz, B Ueberschaer, H Wahlen, M Weierstall, D Wicke

Accelerator CERN-LEP Detector DELPHI

\section{Reactions \\ $e^{t} e^{-} \quad<200 \mathrm{GeV}\left(\mathrm{E}_{\mathrm{cm}}\right)$}

Particles studied $W^{+}, W^{-}, Z^{0}$, hvy-lepton, higgs, hvy-flavor

Brief description A general purpose LEP detector for physics

on and above the $Z^{\circ}$, offering 3-dimensional information on curvature and energy deposition with fine spatial granularity, as well as identification of leptons and hadrons over most of the solid angle. A superconducting coil provides a $1.2 \mathrm{~T}$ solenoidal field of high uniformity. Tracking relies on a microvertex detector, an inner detector, a Time Projection Chamber (TPC), an outer detector, and forward drift chambers. A 3-layer silicon microvertex detector allows a precision measurement of the interaction vertex and decay vertices of short-lived particles such as bottom and charm hadrons and $\tau$ leptons. Electromagnetic showers are measured in the barrel with high granularity by the High Density Projection Chamber (HPC) and in the endcaps by $1^{\circ} \times 1^{\circ}$ projective towers composed of lead glass as an active material and phototriode readout. Hadron identification is provided mainly by liquid and gas ring imaging Cerenkov counters (RICH). A segmented magnet yoke serves for hadron calorimetry and as a filter for muons which are identified in two drift chamber layers. In addition, scintillator systems are implemented in the barrel and forward regions. A small angle Shashlik-type calorimeter (STIC) is used for the luminosity determination. Taking data (July 96).

Journal papers NIM 225 (1984) 477, NIM A225 (1984) 606, NIM A235 (1985) 310, NIM A241 (1985) 429, NIM A243 (1986) 77 , NIM A243 (1986) 91, NIM A248 (1986) 317, NIM A252 (1986) 188, NIM A252 (1986) 413, NIM A252 (1986) 418, NIM A252 (1986) 435, NIM $\Lambda 252$ (1986) 524, NIM A252 (1986) 573, NIM A254 (1987) 111, NIM A256 (1987) 65, NIM A256 (1987) 267, NIM A257 (1987) 499, NIM A260 (1987) 124, IEEE TNS 34 (1987) 227, NIM $A 263$ (1988) 215, NIM A265 (1988) 218, NIM A269 (1988) 652, NIM A270 (1988) 393, NIM A273 (1988) 553, NIM A273 (1988) 565, NIM A273 (1988) 841, NIM A273 (1988) 847 , IEEE TNS 36 (1989) 390, NIM A275 (1989) 49, NIM A277 (1989) 154, NIM A277 (1989) 160, NIM A277 (1989) 338, NIM A277 (1989) 347, NIM A279 (1989) 473, NIM A279 (1989)

518, NIM A283 (1989) 502, NIM A283 (1989) 567, NIM A283 (1989) 792, PL B231 (1989) 539, NIM A289 (1990) 400, NIM A290 (1990) 320, NIM A290 (1990) 327, NIM A292 (1990) 75, NIM A292 (1990) 319, NIM A292 (1990) 551, NIM A294 (1990) 424, PL B240 (1990) 271, PL B241 (1990) 425, PL B241 (1990) 435, PL B241 (1990) 449, PL B242 (1990) 536, PL B245 (1990) 276, PL B247 (1990) 137, PL B247 (1990) 148, PL B247 (1990) 157, PL B247 (1990) 167, PL B252 (1990) 140, PL B252 (1990) 149 , NP B342 (1990) 1, IEEE TNS 38 (1991) 861, NIM A303 (1991) 233, NIM A305 (1991) 344, NIM A310 (1991) 596, PL B255 (1991) 466, PL B260 (1991) 240, PL B267 (1991) 422, PL B268 (1991) 296, NP B367 (1991) 511, ZPHY C50 (1991) 185 , ZPHY C51 (1991) 25, ZPHY C52 (1991) 271, IEEE TNS 39 (1992) 166, NIM A315 (1992) 143, NIM A315 (1992) 393, NIM A323 (1992) 209, NIM A323 (1992) 351, NIM A323 (1992) 363, PL B274 (1992) 230, PL B274 (1992) 498, PL B275 (1992) 222, PL B275 (1992) 231, PL B276 (1992) 247, PL B276 (1992) 254, PL B276 (1992) 536, PL B277 (1992) 37I, PL B281 (1992) 383 PL B286 (1992) 201, PL B289 (1992) 199, PL B295 (1992) 383, PL B298 (1992) 236, PL B298 (1992) 247, NP B373 (1992) 3, NP B386 (1992) 471, ZPHY C53 (1992) 41, ZPHY C53 (1992) 555 , ZPHY C53 (1992) 567, ZPHY C54 (1992) 55, ZPHY C55 (1992) 555, ZPHY C56 (1992) 47, ZPHY C56 (1992) 63, NIM A328 (1993) 447, NIM A338 (1993) 284, PL B301 (1993) 145, PL B302 (1993) 356, PL B307 (1993) 187, PL B307 (1993) 221, PL B311 (1993) 379, PL B311 (1993) 408, PL B312 (1993) 253 PL B316 (1993) 620, PL B318 (1993) 249, NP B403 (1993) 3, ZPHY C57 (1993) 181, ZPHY C59 (1993) 21, ZPHY C59 (1993) 357, ZPHY C59 (1993) 533 [erratum: ZPHY C65 (1995) 709], PL B322 (1994) 459, PL B323 (1994) 242, PL B324 (1994) 500 , PL B327 (1994) 386, PL B332 (1994) 488, PL B334 (1994) 435, PL B338 (1994) 409, PL B341 (1994) 109, NP B417 (1994) 3 


\section{SUMMARIES OF CERN EXPERIMENTS}

[erratum: NP B426 (1994) 244], NP B418 (1994) 403, NP B421 (1994) 3, ZPHY C61 (1994) 407, ZPHY C62 (1994) 357, ZPHY C63 (1994) 3, ZPHY C63 (1994) 17, ZPHY C64 (1994) 183, NIM A362 (1995) 361, PL B342 (1995) 402, PL B345 (1995) 598, PL B347 (1995) 447, PL B355 (1995) 415, PL B357 (1995) 255, PL B357 (1995) 715, PL B359 (1995) 411, PL B361 (1995) 207, NP B444 (1995) 3, ZPHY C65 (1995) 555, ZPHY C65 (1995) 569, ZPHY C65 (1995) 587, ZPHY C65 (1995) 603, ZPHY C66 (1995) 323, ZPHY C66 (1995) 341, ZPHY C67 (1995) 1, ZPHY C67 (1995) 69, ZPHY C67 (1995) 183, ZPHY C67 (1995) 543, ZPHY C68 (1995) 13, ZPHY C68 (1995) 353, ZPIYY C68 (1995) 363, ZPHY C68 (1995) 375, ZPHY C68 (1995) 541, ZPHY C69 (1995) 1, NIM A368 (1996) 314, PL B365 (1996) 448, PL B372 (1996) 172, ZPHY C69 (1996) 223, ZPHY C69 (1996) 561 ,

ZPHY C69 (1996) 575, ZPHY C70 (1996) 179, and ZPHY C70 (1996) 371.

E-mail contact daniel.treille@cern.ch

WWW Home-page http://delinfo.cern.ch/Delphi/Welcome.html

\section{CERN-LEP-L3}

(Proposed 1982, Approved Nov 1982, Began data-taking Aug 1989, In progress)

\section{L3 EXPERIMENT}

\section{L3 COLLABORATION}

AACHEN, TECH HOCHSCH, I PHYS INST \& AACHEN, TECH HOCHSCH, III PHYS INST - R Bock, A Bohm, H Fesefeldt K Hangarter, B Hartmann, W Krenz, K Lubclsmeyer, S Muller, A Nippe, D Pandoulas, C Paus, Y J Pei, S Rohner, S Roth, M Sassowsky, C Schafer, S Schmidt-Kaerst, D Schmitz,

P Schmitz, J Schwenke, G Schwering, T Siedenburg,

A Straessner, M Tonutti, M von der Mey, W Wallraff, A Weber, $S$ Wynhoff, Y Zeng

NIKHEF, AMSTERDAM - G J Bobbink, A P Colijn, P Duinker,

F C Erne, F L Linde, G G G Massaro, D van Dierendonck

MICHIGAN U - T Azemoon, A Button, L W Jones, $R$ Moore, $K$ Riles, B P Roe

ANNECY - G Carlino, X Chereau, G Coignet, A Degre,

D Duchesneau, Y Karyotakis, D Perret-Gallix, S Rosier-Lees, M Vivargent

JOHNS HOPKINS U - C Y Chien, A Gougas, G Hu, D Kim,

A Pevsner

BASEL U - L Tauscher, M Wadhwa

LOUISIANA STATE $U-R R$ MCNeI

BEIJING, IHEP - G Chen, G M Chen, H S Chen, B N Jin,

$\mathrm{Z}$ A Liu, Y S Lu, X W Tang, $K \mathrm{~L}$ Tung, $\mathrm{C} G$ Yang, X Y Yao, Z Zhang, G Y Zhu

HUMBOLDT U, BERLIN - M W Gruenewald, T Hebbeker, $S$ Petrak

BOLOGNA U \& INFN, BOLOGNA - F Anselmo, D Antreasyan,

M Basile, G Cara Romeo, F Cindolo, D Hatzifotiadou

TATA INST - T Aziz, S Banerjee, S Dutta, S N Ganguli,

A Gurtu, M Maity, G Majumder, S Mangla, K Mazumdar,

R Raghavan, S Sarkar, K Sudhakar, S C Tonwar

BOSTON U - S Ahlen, J Goldstein, A Marin, J Xu, B Zhou

NORTHEASTERN U - G Alverson, S S Gau, T Paul, S Reucroft, L Taylor

BUChAREST U - T Angelescu, F Cotorobai, N Gheordanescu, A Mihul

BUDAPEST, CRIP - A Csilling, D Kiss, E Nagy, J Toth, $G$ Vesztergombi

HARVARD U - K Strauch

MIT - U Becker, P Berges, E Brambilla, J D Burger, X D Cai, $M$ Capell, I Clare, R Clare, $T$ S Dai, $P$ de Jong, $F$ J Eppling, P H Fisher, G Forconi, T Kramer, C Lapoint, A Lebedev, D Luckey, S Nahn, H Postema, J Rodin, B Smith, M Steuer, F Sticozzi, S M Ting, S C C Ting ( $\checkmark$ Spokesperson), J C Wang; Y F Wang

FLORENCE U \& INFN, FLORENCE - O Adriani, F Becattini, A M Cartacci, G Castellini, C Civinini, R D'Alessandro, A Favara, G Landi, M Lenti, A Macchiolo, M Meschini, B Monteleoni, G Passaleva, M Pieri, E Pistolesi, P Spillantini CERN - J Allaby, R Barillere, B Bertucci, M Biasini, J J Blaising, A Herve, V Innocente, J Kirkby, M Lebeau, P Lecoq, J M Le Goff, D McNally, J Mnich, D Peach, J A Rubio, J Salicio, U Uwer, F Wittgenstein, A Zichichi
WORLD LAB, GENEVA - U K Chaturvedi, M T Dova, M Kaur, R A Khan, G Sultanov, J D Swain, S X Wu

GENEVA U - G Ambrosi, M Bourquin, W J Burger, C Cecchi,

P Extermann, J H Field, L Fredj, H Hoorani, M N Kienzle-

Focacci, F Muheim, N Produit, D Sciarrino, G F Susinno

HEFEI, CUST - H F Chen, Z F Gong, C Li, W G Ma, L Z Sun,

$X$ L Wang, $Z$ M Wang, $Z$ Z Xu, B Z Yang, J B Ye, Z P Zhang

HELSINKI U - P Laurikainen, R Ostonen, M E Sarakinos

LAUSANNE U - G Alemanni, A Bay, S Goldfarb, A Kasser, $Y \mathrm{Mi}, \mathrm{P}$ Rosselet

LECCE U - F Cesaroni

LOS ALAMOS - J S Kapustinsky, W W Kinnison, J Shukla

LYON, IPN - M Chemarin, H El Mamouni, J P Ernenwein, J Fay, P Lebrun, J P Martin

MADRID, CIEMAT - M Aguilar-Benitez, $\boldsymbol{J}$ Alcaraz, J Berdugo, J Casaus, M Cerrada, M Chamizo, N Colino, B de la Cruz, D Fernandez, I Josa-Mutuberria, P Ladron de Guevara, C Mana, F J Rodriguez, L Romero, E Sanchez

MILAN U \& INFN, MILAN - M Acciarri, A Baschirotto, R Castello, C Furetta, S Pensotti, P G Rancoita, M Rattaggi MOSCOW, ITEP - A Arefiev, Y Galaktionov, A Klimentov,

I Korolko, V Koutsenko, A Kunin, A Malinin, V Plyaskin, V Pojidaev, V Shoutko, E Shumilov, I Vetlitsky, I Vorobiev NAPLES U, IFS \& INFN, NAPLES - A Aloisio, M G Alviggi, N Cavallo, $G$ Chiefari, $R$ de Asmundis, A Doria, E Drago, L Lista, S Mele, L Merola, M Napolitano, P Paolucci, S Patricelli, D Piccolo, C Sciacca

CYPRUS U -- A Hasan, P Razis, A Vorvolakos

NIKHEF, NIJMEGEN \& NIJMEGEN U - A Buytenhuijs,

H De Boeck, W Kittel, A C Konig, H Kuijten, W J Metzger, D J Schotanus, $\mathrm{R} T$ Van de Walle, W $\mathrm{C}$ van Hoek, A $J \mathrm{~W}$ van $\mathrm{Mil}$

OAK RIDGE - H O Cohn, Y Efremenko, Y Kamyshkov, K Read

CAL TECH - G Gratta, A Kirkby, D Kirkby, W Lu, R Mount,

H. Newman, S Shevchenko, A Shvorob, R Y Zhu

PERUGIA U \& INFN, PERUGIA - B Alpat, P Bartalini,

R Battiston, G M Bilei, M Caria, S Easo, E Fiandrini, M Pauluzzi, A Santocchia, L Servoli

CARNEGIE MELLON U - S C Blyth, I C Brock, A Engler, $T$ Ferguson, $F$ Filthaut, $\mathrm{R}$ W Kraemer, H K Park, I C Pinto, II Vogel, J M You

PRINCETON U - P Denes, V K Gupta, P A Piroue,

D P Stickland, H Stone, C Tully

ROME U \& INFN, ROME - P Bagnaia, L Barone, B Borgia,

F Cavallari, S Costantini, F DeNotaristefani, $M$ Diemoz,

C Dionisi, R Faccini, S Falciano, F Ferroni, S Gentile, S Giagu, E Leonardi, E Longo, C Luci, L Ludovici, L Luminari,

L Malgeri, F Marzano, G Mirabelli, S Morganti, G Organtini,

S Paoletti, G Pascale, M Rescigno, E Valente

ST PETERSBURG, INP - V P Andreev, O Fedin, P Levtchenko, D Prokofiev, V Schegelsky, A A Vorobyov, A Zalite, Y Zalite SALERNO U - L Cifarelli

UC, SAN DIEGO - J G Branson, A Dominguez, G Raven SANTIAGO DE COMPOSTELA U - I Duran

SOFIYA, AUTOMATION SCI INSTRUM LAB - N Shivarov, B Stoyanov

KOREA INST SCI, TAEJON - M T Choi, J K Kim, S C Kim, Y G Kim, J S Lee, K Y Lee, S Ro, D Son

ALABAMA U - L Baksay, J Busenitz, D DiBitonto,

$\mathrm{H}$ Tuchscherer

UTRECHT U - A Buijs, T van Rhee, W van Rossum

PURDUE U - K Banicz, L J Gutay, B C Riemers

PSI, VILLIGEN - K Deiters, $M$ Fabre, W Lustermann

DESY-IFH, ZEUTHEN - P Kapinos, R Leiste, E Lieb,

W Lohmann, H Nowak, S Riemann, B Schocneich, A Sopczak, $F$ Tonisch, H Vogt, $\mathrm{R}$ Volkert

ZURICH, ETH - H Anderhub, F Behner, B L Betev, A Biland,

D Bourilkov, V Brigljevic, M Campanelli, F Di Lodovico,

M Dittmar, M Felcini, K Freudenreich, H Hofer, K Lassila-

Pcrini, P Lecomte, P Le Coultre, P Marchesini, F Nessi-

Tedaldi, F Pauss, M Pohl, G Rahal-Callot, D Ren, $\Lambda$ Robohm,

H Rykaczewski, N Scholz, H Suter, J Ulbricht, G Viertel, P Zemp

HAMBURG U - H Schopper

TAIWAN, HEP GROUP - A Chan, Y H Chang, A Chen,

$S \mathrm{R}$ Hou, W $T$ Lin, I C Sens, S C Yeh

Accelerator CERN-LEP Detector L3

Reactions

$$
e^{+} e^{-} \quad<200 \mathrm{GeV}\left(\mathrm{E}_{\mathrm{cm}}\right)
$$




\section{SUMMARIES OF CERN EXPERIMENTS}

Particles studied $Z^{\circ}, W^{ \pm}, \gamma$, quark, giuon, leptons, hvy-lepton, higgs, s-particle

Brief description The detector consists of a high-valume low. field solenoid magnet, a small central tracking chamber with very high spatial resolution, a high-resolution electromagnetic calorimeter encapsulating the central detector, a hadron calorimeter acting also as a muon filter, and high-precision muon tracking chambers. The detector is designed to measure energy and position of leptons with the highest obtainable precision allowing a mass resolution $\Delta m / m$ smaller than $2 \%$ in dilepton final states. Hadronic energy flux is detected by a fine-grained calorimeter, which also serves as a muon filter and a tracking device. The outer boundary of the detector is given by the iron return-yoke of a conventional magnet. The ficld is $0.5 \mathrm{~T}$ over a length of $12 \mathrm{~m}$. The muon momentum measurement is performed by three sets of drift chambers in the central detector region. A forward-backward muon detection system extends the polar angle coverage to 22 degrees in the forward region. Radially inwards is a combined hadron calorimeter and muon absorber. The electromagnetic cnergy flow is determined by approximately 11000 crystals of BGO. Full electromagnetic shower containment over nearly $4 \pi$ solid angle coverage is achieved. Surrounding the $10 \mathrm{~cm}$ diameter beam pipe, a highprecision Silicon Microstrip Detector (SMD) and a small drift chamber operating in the time expansion mode (TEC) act as charged particle vertex detectors. Taking data (July 96).

Journal papers NIM 214 (1983) 525, NIM A225 (1984) 493, NIM A228 (1985) 294, NIM A235 (1985) 464, NIM A251 (1986) 258, NIM A252 (1986) 304, CPC 45 (1987) 391, NIM A253 (1986) 15, NIM A254 (1987) 535, NIM A256 (1987) 261, NIM A257 (1987) 125, NIM $A 257$ (1987) 528, HEPNP 12 (1987) 587, NIM A258 (1987) 58, NIM A263 (1988) 14, NIM A263 (1988) 343, NIM A265 (1988) 50, NIM A265 (1988) 252, NIM A270 (1988) 397 NIM A272 (1988) 713, NIM A273 (1988) 471, NIM A273 (1988) 814, NIM A274 (1989) 113, NIM A275 (1989) 71, NIM A275 (1989) 81, NIM A277 (1989) 187, NIM A278 (1989) 699, NIM A279 (1989) 671, NIM A280 (1989) 25, NIM A283 (1989) 799 NIM A285 (1989) 403, PL B231 (1989) 509, PL B233 (1989) 530, MPL A5 (1990) 1381, NIM A288 (1990) 364, NIM A289 (1990) 35, NIM A289 (1990) 103, NIM A289 (1990) 335, NIM A290 (1990) 115, PL B236 (1990) 109, PL B237 (1990) 136, PL $\mathrm{B} 238$ (1990) 122, PL B241 (1990) 416, PL B247 (1990) 177, PL B247 (1990) 473, PL B248 (1990) 203, PL B248 (1990) 227 , PL B248 (1990) 464, PL B249 (1990) 341, PL B250 (1990) 183, PL B250 (1990) 199, PL B250 (1990) 205, PL B251 (1990) 311, PL B251 (1990) 321, PL B252 (1990) 511, PL B252 (1990) 518, PL B252 (1990) 525, PL B252 (1990) 703, PL B252 (1990) 713, NIM A302 (1991) 53, NIM A306 (1990) 150, NIM A309 (1991) 318, PL B257 (1991) 450, PL B257 (1991) 469, PL B259 (1991) 199 PL B261 (1991) 169, PL B261 (1991) 177, PL B262 (1991) 155 , PL B263 (1991) 551, PL B265 (1991) 451, PL B270 (1991) 111 PL B271 (1991) 453, PL B271 (1991) 461, ZPHX C51 (1991) 179, PL B275 (1992) 209, PL B276 (1992) 247, PL B283 (1992) 454, PL B284 (1992) 471, PL B286 (1992) 403, PL B288 (1992) 395, PL B288 (1992) 404, PL B288 (1992) 412, PL B292 (1992) 454, PL B292 (1992) 463, PL B292 (1992) 472, PL B294 (1992) 457, PL B294 (1992) 466, PL B295 (1992) 337, PL B295 (1992) 371, PL B297 (1992) 469, ZPHY C55 (1992) 39, PL B301 (1993) 136, PL B303 (1993) 391, PL B306 (1993) 187, PL B307 (1993) $187, \mathrm{PL} B 307$ (1993) 237, PL B309 (1993) 451, PL B313 (1993) 326, PL B315 (1993) 494, PL B316 (1993) 427, PL B317 (1993) 467, PL B317 (1993) 474, PL B317 (1993) 637, PL B318 (1993) 575, PRPL 236 (1993) 1, ZPHY C57 (1993) 355, NIM A340 (1994) 396, NIM A343 (1994) 456, NIM A344 (1994) 133, NIM A344 (1994) 212, NIM A344 (1994) 521, NIM A348 (1994) 431, NIM A348 (1994) 436, NIM A351 (1994) 300, IEEE TNS 41 (1994) 772, PL B321 (1994) 283, PL B328 (1994) 223, PL B332 (1994) 201, PL B335 (1994) 542, PL B341 (1994) 245, ZPHY C62 (1994) 551, PL B345 (1995) 74, PL B345 (1995) 93, PL B345 (1995) 589, PL B345 (1995) 609, PL B346 (1995) 190, PI $\mathrm{B} 350$ (1995) 109, PL B351 (1995) 375, PL B352 (1995) 487, PL B353 (1995) 136, PL B353 (1995) 145, PL B363 (1995) 118, PL $\mathrm{B} 363$ (1995) 127, PL B363 (1995) 137, PL B370 (1996) 195, PL B370 (1996) 211, PL B371 (1996) 126, PL B37I (1996) 137, and PL B374 (1996) 331 .

E-mail contact david.stickland@cern.ch WWW Home-page http://hpl3sn02.cern.ch/

\section{CERN-LEP-OPAL}

(Proposed 1982, Approved Nov 1982, Began data-taking Aug 1989, In progress)

\section{THE OPAL DETECTOR (AN OMNI PURPOSE APPARATUS FOR LEP)}

OPAL COLLABORATION

AACHEN, TECH HOCHSCH, III PHYS INST - J Bechtluft,

$S$ Bethke, O Biebel, D Lanske, P Pfeifenschneider, U Ruppel

ALBERTA U -- D Gingrich, J Hewlett, J McDonald, S Mullin,

$\mathrm{J}$ Pinfold, H Przysiczniak, N Rodning, P Routenburg, I Schaapman, W Springer

BIRMINGIIAM U - A N Bell, I J Bloodworth, J Bloomer, D G Charlton, S J Hillier, R J Homer, M Jimack, P Jovanovic, 'I' J Mc Mahon, S W O'Neale, M Pearce, D Rees, D Rigby, $S D$ ralbot, $P$ Matkins, A $T$ Watson, $J A$ Wilson

BOLOGNA U \& INFN, BOLOGNA - S Arcelli, P Capiluppi, M Cuffiani, G M Dallavalle, F Fabbri, M Ferrari, M Fierro, G Giacomelli, R Giacomelli, C Grandi, J Letts, S Marcellini, A Michelini, A Montanari, F Odorici, B Poli, A M Rossi, C Sbarra, G P Siroli

BONN U - R Bartoldus, K Desch, A Eyring, H M Fischer, C Geich-Gimbel, S Gross, C Hartmann, 'I P Kokott, P Maettig, C Markus, S Menke, U C Mueller, B Nellen, A Posthaus, F Scharf, P Schuetz, M Schumacher, A Stahl, B Stockhausen, $E$ von Toerne, $N$ Wermes

BUDAPEST, CRIP - C Hadju, D Horvath, G Pasitor

CAMBRIDGE U - N Altekamp, J R Batley, I R Carter, V Gibson, M J Goodrick, C M Hawkes, J C Hill, D E Hutchcroft, C R Jones, R Shaw, C P Ward, D R Ward, $S$ Wotton

CARLETON U - R K Carnegie, C Charlesworth, P E Estabrooks, R J Hemingway, M Jones, D Karlen, D Koetke, P Krieger, $S$ Towers, P Weber

CERN - E Barberio, T Behnke, S Bentvelsen, V Blobel, H J Burckhart, C Burgard, L Del Pozo, A Fuertjes, W Glessing, M. Gruwe, M Guillot, $R$ Hammarstrom, $M$ Hansroul, $M$ Hauschild, $R$ Hawkings, $R D$ Keuer ( $\checkmark$ Spokesperson), $M$ Hildreth, J Hill, M Jimenez, $\mathrm{R}$ W L Jones, 'T R Junk, B King, S Kluth, R Kowalewski, R Lorenzi, M Mannelli, R A McPherson, F Meijers, $H$ A Neal, $R$ Nisius, D Plane, A Renoux, E Ros, O Runolfsson, P Scharff-Hansen, B Schmitt, M Schrocder, M Schulz, A M Smith, F Strumia, M Tecchio, M Thomson, M Turner-Watson, P Wells, K Zankel, M Znoy CHICAGO U - K J Anderson, R L Armstrong, H Evans, D Glenzinski, P Hart, F \& Merritt, M J Oreglia, J E Pilcher, E Pod, H Sanders, A Turcot

DEBRECEN, INST NUCL RES . J Palinkas

DUKE U - S Lautenschlager, $A$ Lee

FREIBURG U - P Berlich, M Bobinski, R Buergen, G Herten T Hilse, D Joos, M Kobel, R Kolpin, M Kolrep, J Ludwig, W Mohr, J Patt, H Roescr, K Runge, K Sachs, H SchultzCoulon, S Soeldner-Rembold, M Thiergen, M Verzocchi, $\mathrm{H} \mathrm{C}$ Weber, B Wilkens

TECHNION - S Dado, J Goldberg, D Lazic, N Lupu, Y Rozen. $\mathrm{S}$ Tarem

DESY \& HAMBURG U - J Meyer, S Petzold, C H ShepherdThemistocleous, A Sittler, J Steuerer, A Wagner, G Wilson HEIDELBERG U, IHEP - P Bock, H M Bosch, P Fath, P IgoKemenes, U Jost, 'T Kress, S Schmitt, V Soergel, M Steiert, P Utzat, J von Krogh, $P$ von Walter

INDIANA $U$ - S Anderson, B Bouwens, S Braibant, S De Jong, $\mathrm{E}$ do Couto e Silva, F Fiedler, G Hanson, H O Ogren, D R Rust, M Starks, $\mathrm{R}$ van Kooten

KOBE U - K Kawagoe, H Takeda

UNIVERSITY COLL, LONDON - B E Anderson, D J Attree, A Charalambous, P E L Clarke, J E Conboy, $R$ Cranfield, $M$ Cresswell, D $A$ Hayes, $J$ W Lauber, C Lewis, E A McKigney, D I Miller, T Rooke, P Sherwood, A Skillman, J J Ward

BRUNEL U - K Ametewec, P Hobson, D C Imrie, W Matthews, R Rylko

QUEEN MARY - WES TFIELD COLL - G A Beck, A A Carter M D Evans, W R Gibson, M Hapke, P Kyberd, S L Lloyd, A J Martin, A I McNab, J Morris, T W Pritchard, $S$ A Robins MANCHESTER U - J Allison, R J Barlow, C Beeston, $S G$ Clowes, O Cooke, I P Duerdoth, J Edwards, R E HughesJones, G D Lafferty, F K Locbinger, B Nijjhar, $N$ J Oldershaw, J Pater, P D Phillips, K Stephens, E Vokurka, T R Wyatt 


\section{SUMMARIES OF CERN EXPERIMENTS}

MARYLAND U - A Ball, R Bard, C Y Chang, C Dallapiccola, D Fong, M Foucher, S Gascon-Shotkin, A Jawahery, R G Kellogg, R Lahmann, G Long, G Martinez, A Skuja, G A Snow

MONTREAL U - G Azuelos, G Beaudoin, M Beaulieu, M Doucct, $J$ Gascon, H Jeremie, A Joly, E Lefebvre, J-P Martin, $R$ Tafirout, $P$ Taras, $P$ Vikas, V Zacek

MUNICH U - M Boutemeur, G Duckeck, A D Schaile, O Schaile OREGON U - W-P Lai, D Strom

CRPP, OTTAWA - M S Dixit, P Gagnon, C K Hargrove, M J Losty, H Mes, F G Oakham, N K Watson

UC, RIVERSIDE - D Chrisman, S L Chu, J W Gary, P Giacomelli, W Gorn, J G Layter, P Schenk, B C Shen RUTHERFORD - K W Bell, R M Brown, N I Geddes, T Geralis, F R Jacob, P W Jeffreys, B W Kennedy, G N Patrick, W G Scott, M Sproston, P Szymanski

DAPNYA, SACLAY - A Gaidot, F-X Gentit, H Lafoux, A Malik, $G$ Vasseur

TEL AVIV U .. G Alexander, G Bella, I Cohen, E Sarkisyan, E Tsur

TOKYO U - S Asai, K Ishii, I Kanzaki, T Kawamoto,

T Kobayashi, S Komamiya, T Mashimo, ' $\mathrm{I}$ Mori, M Morii,

I Nakamura, $\mathrm{T}$ Omori, S Orito, S Tanaka, 'T T'sukamoto, S Yamashita

BRI'TISH COLUMBIA U - D A Axen, R Howard, J Kirk,

3 McKenna

VICTORIA U - D Deatrich, A Honma, R K Keeler, I Lawson, $P$ Poffenberger, S Robertson, M J Roney, M Rosvick, $T$ I Smith, $R$ Sobie, $M$ Vincter, $J$ White

WEIZMANN INST - E Duchovni, R Folman, E Gross, D Lellouch, L Levinson, G Mikenberg, R Mir, K Nagai, M Shoa, G Wolf, $G$ Yekutieli

Accelerator CERN-LEP Detector OPAL

Reactions

$$
e^{+} e^{-} \quad<200 \mathrm{GeV}\left(\mathrm{E}_{\mathrm{cm}}\right)
$$

Particles studied $Z^{0}, W^{+}, W^{-}, \gamma, \tau$, hvy-flavor, gluon, higgs,

s-particle

Brief description OPAL is a general purpose detector designed to study a wide range of unexplored physics at LEP. Among the central physics issues are the study of the $Z^{0}$ and $W^{ \pm}$bosons (e.g., the determination of their exact masses and widths, and couplings to leptons and quarks), the physics of hcavy flavors (such as the spectroscopy of $b$ quarks and the determination of the mixing and lifetimes of $B$ states), and various QCD topics. $A$ general search for new particles, in particular the Higgs bosons, is being made. The main components of the apparatus, in order of increasing distance from the interaction point, are a silicon microvertex detector, central detectors consisting of a vertex and a jet chamber, and a barrel of $Z$ chambers, a warm conductor solenoid providing a uniform magnetic field of $0.4 \mathrm{~T}$, a TOF scintillator barrel detector complimented by a scintillating tile endcap detector, a $4 \pi$ lead glass electromagnetic calorimeter, a hadron calorimeter instrumented by streamer tubes and thin gap wire chambers, an external muon identifier, and a forward detector which includes a new small-angle silicontungsten calorimeter. Taking data (July 96).

Journal papers NIM A236 (1985) 284, IEEE TNS 32 (1985) 736, NIM A242 (1986) 247, NIM A244 (1986) 416, NIM A250 (1986) 503, NIM A252 (1986) 331, NIM A252 (1986) 511, IEEE TNS 34 (1987) 240, CPC 47 (1987) 55, NIM A260 (1987) 132, NIM A260 (1987) 329, NIM A265 (1988) 11, NIM A265 (1988) 445, IEEE TNS 36 (1989) 380, NIM A278 (1989) 725, NIM A279 (1989) 236, NIM A279 (1989) 523, NIM A283 (1989) 492, NIM A283 (1989) 515, NIM A283 (1989) 650, PL B231 (1989) 530, IEEE TNS 37 (1990) 1584, NIM A286 (1990) 99, NIM A286 (1990) 107, NIM A286 (1990) 117, NIM A290 (1990) 76, NIM A293 (1990) 145 , NIM A294 (1990) 431, PL B235 (1990) 379, PL B235 (1990) 389, PL B236 (1990) 224, PL B236 (1990) 364 , PL B240 (1990) 250, PL B240 (1990) 261, PL B240 (1990) 497, PL B241 (1990) 133, PL B242 (1990) 299, PL B244 (1990) 135, PL B246 (1990) 285, PL B247 (1990) 448, PL B247 (1990) 458, PL B247 (1990) 617, PL B248 (1990) 211, PL B251 (1990) 211, PL B252 (1990) 159, PL B252 (1990) 290, ZPHY C47 (1990) 505 NIM A302 (1991) 434, NIM A305 (1991) 275, NIM A310 (1991) 527, PL B253 (1991) 511, PL B254 (1991) 293, PL B257 (1991) 531, PL B261 (1991) 334, PL B262 (1991) 341, PL B262 (1991) 351, PL B263 (1991) 123, PL B263 (1991) 311, PL B264 (1991)
219, PL B264 (1991) 467, PL B265 (1991) 462, PL B266 (1991) 201, PL B266 (1991) 485, PL B267 (1991) 143, PL B268 (1991) 122, PL B273 (1991) 338, PL B273 (1991) 355, ZPHY C49 (1991) 1, ZPHY C49 (1991) 49, ZPHY C49 (1991) 375, ZPHY C50 (1991) 373, ZPHY C52 (1991) 175, ZPHY C52 (1991) 543 , NIM A313 (1992) 103, NIM A314 (1992) 74, NIM A317 (1992) 47, NIM A320 (1992) 183, NIM A323 (1992) 169, PL B274 (1992) 513, PL B276 (1992) 247, PL B276 (1992) 379, PL B276 (1992) 547 , PL B278 (1992) 485, PL B281 (1992) 394, PL B281 (1992) 405, PL B287 (1992) 389, PL B287 (1992) 401, PL B288 (1992) 373, PL B291 (1992) 503, PL B294 (1992) 436, PL B295 (1992) 347, PL B295 (1992) 357, ZPHY C53 (1992) 539, ZPHY C54 (1992) 193, ZPHY C55 (1992) 1, ZPHY C55 (1992) 191, ZPHY C56 (1992) 521, NIM A324 (1993) 34, NIM A325 (1993) 129, NIM A325 (1993) 271, NIM A325 (1993) 494, NIM A333 (1993) 330, PL B298 (1993) 456, PL B302 (1993) 523, PL B305 (1993) 407, PL B305 (1993) 415, PL B307 (1993) 187, PL B307 (1993) 247, PL B311 (1993) 391, PL B312 (1993) 501, PL B313 (1993) 333, PL B316 (1993) 435, ZPHY C58 (1993) 207, ZPHY C58 (1993) 219, ZPHY C58 (1993) 387, ZPHY C58 (1993) 405, ZPHY C58 (1993) 523, ZPHY C59 (1993) 1, ZPHY C59 (1993) 183, ZPHY C60 (1993) 19, ZPHY C60 (1993) 199, ZPHY C60 (1993) 217, ZPHY C60 (1993) 397, ZPHY C60 (1993) 579 , ZPHY C60 (1993) 593, ZPHY C60 (1993) 601, NIM A348 (1994) 409, NIM A348 (1994) 421, NIM A348 (1994) 424, PL B320 (1994) 417, PL B327 (1994) 397, PL B327 (1994) 411, PL B328 (1994) 207, PL B336 (1994) 585, PL B337 (1994) 196, PL B337 (1994) 207, PL B337 (1994) 393, PL B338 (1994) 497, PL B339 (1994) 278, NC 107A (1994) 2007, NC 107A (1994) 2055, ZPHY C61 (1994) 19, ZPHY C61 (1994) 199, ZPHY C61 (1994) 209, ZPHY C61 (1994) 357, ZPHY C63 (1994) 181, ZPHY C63 (1994) 197, ZPHY C63 (1994) 363, ZPHY C64 (1994) 1, PL B350 (1995) 273, PL B352 (1995) 176, PL B353 (1995) 402, PL B353 (1995) 595, PL B358 (1995) 162, PL B364 (1995) 93 ZPHY C65 (1995) 1, ZPHY C65 (1995) 17, ZPHY C65 (1995) 31 , ZPHY C65 (1995) 47, ZPHY C65 (1995) 183, ZPHY C65 (1995) 367, ZPHY C66 (1995) 19, ZPHY C66 (1995) 31, ZPHY C66 (1995) 543, ZPHY C66 (1995) 555, ZPHY C67 (1995) 15 ZPHY C67 (1995) 27, ZPHY C67 (1995) 45, ZPHY C67 (1995) 57, ZPHY C67 (1995) 203, ZPHY C67 (1995) 365, ZPHY C67 (1995) 379, ZPHY C67 (1995) 389, ZPHY C67 (1995) 555, ZPHY C68 (1995) 1, ZPHY C68 (1995) 179, ZPHY C68 (1995) 203, ZPHY C68 (1995) 519, ZPHY C68 (1995) 531, ZPHY C68 (1995) 555, PL B368 (1996) 244, PL B369 (1996) 163, PL B370 (1996) 174, PL B370 (1996) 185, PL B374 (1996) 341, PL B376 (1996) 232, PL B376 (1996) 315, ZPHY C69 (1996) 195, ZPHY C69 (1996) 543, ZPHY C70 (1996) 197, ZPHY C70 (1996) 357, and ZPHY C71 (1996) 1 .

E-mail contact rolf.heuer@cern.ch WWW Home-page http://www1.cern.ch/Opal/

\section{CERN-LEP-06}

(Proposed 1988, Approved Sep 1989, Began data-taking 1990, Completed data-taking Feb 1992)

\section{THE SEARCH FOR HIGHLY IONIZING PARTICLES IN} $e^{+} e^{-}$COLLISIONS AT LEP USING MODAL

L6-MODAL COLLABORATION

ALBERTA U - J L Pinfold ( $\sqrt{ }$ Spokesperson)

BOLOGNA U \& INFN, BOLOGNA -. G Giacomelli, F Patrizii, F Predicri, P Serra

HARVARD U - K Kinoshita

Accelerator CERN-LEP Detector Plastic

Reactions

$$
e^{+} e^{-} \rightarrow \quad 90 \mathrm{GeV}\left(\mathrm{E}_{\mathrm{cm}}\right)
$$

Particlesstudied monopole

Brief description The MODAL (MOnopole Detector At LEP) detector is designed to search for monopoles, dyons, and other highly ionizing particles. It is formed from lexan/CR39 diclectric track detector modules arranged in a polyhedral configuration around the intersection region.

Journal papers PR D46 (1992) 881.

E-mail contact james.pinfold@cern.ch 


\section{SUMMARIES OF CERN EXPERIMENTS}

\section{CERN-NA-012-2}

(Proposed Aug 1985, Approved Feb 1986, Began data-taking Oct 1986, Completed data-taking Nov 1993)

SEARCH FOR MESONS AND GLUEBALLS DECAYING INTO MULTIPHOTON FINAL STATES PRODUCED IN CENTRAL HADRON COLLISIONS AND STUDY OF INCLUSIVE PRODUCTION OF HEAVY QUARK MESONS

GAMS COLLABORATION

ANNECY - T Kinashi, J P Peigneux, M Poulet

KEK - S Inaba, M Kobayashi, T Tsuru

LOS ALAMOS - D Alde, E A Knapp

PISA U \& INFN, PISA - R Bellazzini, A Brez, M M Massai, M R Torquati

SERPUKHOV - S V Donskov, A V Inyakin, G V Khaustov,

A V Kulik, A A Lednev, S A Polovnikov, V A Polyakov,

YD Prokoshkin ( $\checkmark$ Spokesperson), S A Sadovsky,

V D Samoylenko, P M Shagin, A V Shtannikov, A V Singovsky, V P Sugonyaev

BRUSSELS U \& BRUSSELS U, IISN - F Binon, J P Stroot

( $\checkmark$ Spokesperson)

CHIBA U - H Kawai

MIYAZAKI U - T Nakamura, K Takamatsu

YAMAGATA U - H Shimizu

CERN - M Boutemeur

Accelerator CERN-SPS Detector GAMS-4000

Reactions

$\begin{array}{lc}\pi^{-} p \rightarrow p \pi^{-} 2 \gamma\left(\gamma^{\prime} \mathrm{s}\right) & 300 \mathrm{GeV} / c \\ \pi^{-} p \rightarrow 2 \gamma\left(\gamma^{\prime} \mathrm{s}\right) \mathrm{X} & " \\ \pi^{--} n \rightarrow n \pi^{-} 2 \gamma\left(\gamma^{\prime} \mathrm{s}\right) & " \\ \pi^{-} n \rightarrow 2 \gamma\left(\gamma^{\prime} \mathrm{s}\right) \mathrm{X} & " \\ p p \rightarrow 2 p 2 \gamma\left(\gamma^{\prime} \mathrm{s}\right) & 450 \mathrm{GeV} / c\end{array}$

Particles studied glueball, exotic, meson ${ }^{0}, \eta_{c}(1 S), \chi_{c}$ (unspec)

Brief description Searches for neutral mesons, exotics like glueballs, hybrids, and many-quark states produced in central hadron-proton collisions. Studies the inclusive hadronic production of neutral heavy quark mesons. Uses the 4092-cell electromagnetic calorimeter GAMS- 4000 supplemented with a forward magnetic spectrometer and microstrip gas chambers. Data analysis in progress ( $J$ uly 96 ).

Journal papers NIM A268 (1988) 112, NIM A269 (1988) 101, PL B201 (1988) 160, YF 47 (1988) 1273, YF 47 (1988) 1639, NIM A276 (1989) 652, YF 49 (1989) 712, ZPHY C43 (1989) 541, DANS 316 (1991) 900, NIM A315 (1992) 21, NIM A342 (1994) 389, PL B340 (1994) 122, NC A107 (1994) 1867, NC A.107 (1994) 1911, ZPHY C61 (1994) 35, YF 58 (1995) 662, DANS 344 (1995) 469, ZPHY C66 (1995) 375 , and ZPHY C66 (1995) 379.

Related experiments CERN-NA-012, CERN-WA-091, CERNWA-102

E-mail contact prokoshkin@mx.ihep.su, jean-pierre.stroot@cern.ch

\section{CERN-NA-045-2}

(Proposed Jan 1994, Approved Apr 1995, Began data-taking Nov 1995, In progress)

STUDY OF LOW-MASS ELECTRON PAIR AND PHOTON PRODUCTION IN LEAD-LEAD COLLISIONS

CERES COLLABORATION

BROOKHAVEN - P Holl, H Kraner, P Rehak

CERN - J Schukraft

DUBNA - G Agakichiev, Y Minaev, Y Panebrattsev, S Razin,

S Shimanski, V Yurevich

DARMSTADT, GSI - P Braun-Munzinger, D Miskowiec

HEIDELBERG, MAX PLANCK INST - F Ceretto,

U Faschingbauer, C Fuchs, M Hemberger, O Nix, J P Wurm

HEIDELBERG U, IHEP - $R$ Baur, C Bormann, A Drees,

S Esumi, P Glaessel, C H Jung, B Lenkeit, M Messer, A Pfeiffer,

H J Specht, J Stachel, T'S Ullrich, C Voigt, I P Wessels
MILAN POLYTECHNIC - E Gatti, M Sampietro

WEIZMANN INST - C P de los Heros, Z Fraenkel, I Ravinovich, E Socol. G Tel-Zur, I Tserruya ( $\checkmark$ Spokesperson)

Accelerator CERN-SPS Detector Ring imaging Cerenkov, Drift chamber, Multiwire proportional chamber

$\underline{\text { Reactions }}$

${ }^{207} \mathrm{~Pb}$ nucleus $\rightarrow e^{+} e^{-} \mathrm{X}$

${ }^{207} \mathrm{~Pb}$ nucleus $\rightarrow e^{+} e^{-} \gamma \mathrm{X}$

$160 \mathrm{GeV} / \mathrm{c}\left(\mathrm{P}_{\mathrm{lab}} / \mathrm{N}\right)$

Brief description A continuation of the CERN-NA-045 exper-

iment dedicated to the measurement of $e^{+} e^{-}$pairs and direct photons produced in nucleus-nucleus collisions. The main goal is the study of the $e^{+} e^{-}$pair continuum in the invariant mass region from $50 \mathrm{MeV} / \mathrm{c}^{2}$ up to $2 \mathrm{GeV} / \mathrm{c}^{2}$ in a search for thermal emission from the conjectured quark-gluon plasma. It also addresses the question of chiral symmetry restoration in high density matter through the study of the vector mesons $\rho, \omega$ and $\phi$. The CERES spectrometer has been upgraded to cope with the higher multiplicities and background of the central $\mathrm{Pb}$-nucleus collisions. The basic spectrometer consists of two ring imaging Čerenkov (RICH) detectors separated by a superconducting double solenoid. The main elements of the upgrade are additional detectors, two silicon radial-drift chambers (instead of one in the original setup) and a pad chamber (a large MWPC with pad readout) located behind the spectrometer. They allow real tracking and help the pattern recognition of the RICH detectors, by providing an a priori knowledge of the ring location in the UV counters. The two silicon radial-drift chambers allow precise vertex reconstruction and provide rapidity density information. They also play, together with the first RICH, a major role in the rejection of $\gamma$ conversions and $\pi^{0}$-Dalitz decays. The upgrade includes also a new $D A Q$ system with a rate capability higher by more than one order of magnitude compared to the one used in CERN-NA-045. Taking data (July 96).

Journal papers Includes the list of papers by the CERN-NA-045 experiment. IEEE TNS 35 (1988) 404, IEEE TNS 35 (1988) 432, NIM A273 (1988) 798, IEEE TNS 37 (1990) 241, IEEE TNS 39 (1992) 619, NIM A316 (1992) 259, NIM A326 (1993) 273, NP A553 (1993) 857c, NIM A343 (1994) 87, NIM A343 (1994) 231 NP A566 (1994) 87c, NP A566 (1994) 347c, PL B332 (1994) 471 NIM A355 (1995) 329, NP A590 (1995) 103c, and PRL 75 (1995) 1272 .

Related experiments CERN-NA-045

E-mail contact fntsruya@weizmann.weizmann.ac.il, itzhak.tserruya@cern.ch

WWW Home-page http://ceres6.physi.uni-heidelberg.de/

\section{CERN-NA-047}

(Proposed Dec 1988, Approved Apr 1989, Began data-taking Aug 1991, In progress)

MEASUREMENT OF THE SPIN DEPENDENT STRUCTURE FUNCTIONS OF THE NEUTRON AND PROTON

SPIN MUON COLLABORATION (SMC)

NIKHEF, AMSTERDAM - $\mathrm{N}$ de Groot, $\mathrm{T}$ J Ketel, E Kok,

$M$ Litmaath, J E J Oberski, H Postma, E P Sichtcrmann,

R van Dantzig, $G$ van Middelkoop

BIELEFELD U - G Baum, S Bueltmann, D Kraemer

CERN - P Hautle, C A Heusch, W Kroeger, J-M Le Goff,

T O Niinikoski, U Stiegler, $R$ Voss

DUBNA - A Karev, Y Kisselev, V Krivokhijine, K Medved, A Nagajcev, D Peshekhonov, D Pose, I Savin, G Smirnov FREIBURG U - U Landgraf, A Witzmann

GKSS, GEESTHACHT - H Stuhrmann, R Willumeit, J Zhao

HELSINKI U OF TECH - P Berglund, J Kyynarainen, J Ylostalo

BOGAZICI U - E Arik, T Cuhadar, E Gulmez

ISTANBUL, TECH U - T Akdogan, H Guven, C Ozben.

I Reyhancan, G Unel

MAINZ U - E M Kabuss, G K Mallot, J Pretz, R Seitz,

A Steinmetz, D von Harrach

MONS U - $\mathrm{R}$ Windmolders

MUNICH U -. L Betev, A Staude, J Vogt 


\section{SUMMARIES OF CERN EXPERIMENTS}

NAGOYA U - T Hasegawa, N Hayashi, N Horikawa, S Ishimoto, T Iwata, T Kageya, A Kishi, T Matsuda, K Mori, A Ogawa NOR'THEASTERN U -. J Moromisato, E von Gocler NORTHWESTERN U - D Fasching, D Miller, R Segel, P Shanahan, $M$ Velasco

RICE U - B E Bonner, J Cranshaw, S Eichblatt, T Gaussiran, $M$ Lowe, J B Roberts

SACLAY - N de Botton, E Burtin, A de Lesquen, F Feinstein B Frois, F Lehar, A Magnon, F Marie, J Martino, F PerrotKunne, S Platchkov

SANTIAGO DE COMPOSTELA U - B Adeva, C Fernandez, J A Garzon, A Gomez, G Gracia, S Lopez-Ponte, C A Perez, M Plo, J Saborido

TEL AVIV U - J Lichtenstadt, I Sabo

TRIESTE U, IST FIS \& INFN, TRIESTE - R Birsa,

F Bradamante, A Bressan, M Clocchiatti, S Dalla Torre,

M Giorgi, M Lamanna, A Martin, A Penzo, R Puntaferro,

P Schiavon, F Simeoni, F Tessarotto, A Zanett

UCLA - B Derro, C Dulya, G Igo, C Whitten

HOUSTON U - B Mayes, L Pinsky, J Pyrlik, R Weinstein

UPPSALA $U$ - A Arvidson, P Bjorkholm, A Dyring, $M$ Rodriguez

VIRGINIA TECH - D Crabb, J McCarthy

WARSAW, INST NUCL STUDIES - B Badelek, J Kiryluk,

K Kurek, J Polec

SOLTAN INST, SWIERK - J Nassalski, E Rondio, A Sandacz, M Szleper, W Wislicki

YALE U - A Deshpande, S Dhawan, V W Hughes (Spokesperson), R Piegaia

Accelerator CERN-SPS Detector EMC

Reactions Polarized beam and target

muon $p \rightarrow$ muon $\mathrm{X} \quad 100-200 \mathrm{GeV}\left(\mathrm{E}_{\mathrm{lab}}\right)$

muon deut $\rightarrow$ muon $\mathrm{X}$

Particles studied $p, n$, deut

Brief description Measurcs the spin-dependent asymmetries $A_{1}$ and $A_{2}$ in deep inelastic scattering of longitudinally polarized muons by longitudinally and transversely polarized protons and deuterons. Tests the nucleon spin structure and EllisJaffe and Bjorken sum rules. The apparatus is the upgraded forward spectrometer which was used originally by the European and New Muon Collaborations. To minimize the systematic uncertainties the target contains two oppositely polarized cells, which are exposed to the muon beam simultancously. 'The polarized target has a high cooling power and field homogeneity, and long target cells. It was used with proton (butanol beads) and deuteron target materials in previous years, and with proton material (ammonia) in 1996. Taking data (June 96).

Journal papers PL B302 (1993) 533, NIM A343 (1994) 400, NIM A349 (1994) 334, PL B320 (1994) 400, PL B329 (1994) 399, PL B336 (1994) 125, and PL B369 (1996) 93.

Related experiments CERN-NA-002, CERN-NA-009, CERN-NA028, CERN-NA-037

E-mail cantact vernon.hughes@yale.edu

WWW Home-page http://na47sun05.cern.ch/welcome.html

\section{CERN-NA-048}

(Proposed Jul 1990, Approved Nov 1991, In preparation)

A PRECISION MEASUREMENT OF $\epsilon^{\prime} / \epsilon$ IN $C P$ VIOLATING $K^{0} \rightarrow 2 \pi$ DECAYS

NA48 COLLABORATION

SACLAY - J Alitti, S Anvar, D Bederede, F Bugcon, J-B Cheze,

$M$ De Beer, P Debu, I L Fallou, A Givernaud, H Le Provost,

F Louis, E Mazzucato, A Migliori, M Mur, B Peyaud,

$S$ Schanne, G Tarte. $R$ Turlay, B Vallage

CERN - F Bal, G D Barr, G Bocquet, P Buchholz, D Cundy.

N Doble, F Formenti, W Funk, L Gatignon, A Gonidec,

P Grafstrom, B Klallgren, W Ywanski. G Kesseler, A Lacourt,

G Laverriere, G Linser, M Martini, M Mast, J P Matheys,

A Norton, P Ponting, D Schinzel, W Seidl, M Sozzi, H Taureg,

$\mathrm{P}$ Vande Vyvre, $\mathrm{M}$ Velasco, $\mathrm{O}$ Vossnack, $\mathrm{H}$ Wahl, $\mathrm{P}$ Wertelaers.

J Wetering, $\mathrm{M}$ Ziolkowski

CAGLIARI U \& INFN. CAGLIARI - V Fanti, A Lai, L Musa,

A Nappi. P Randaccio, M-G Setzu
CAMBRIDGE U - S Katvars, R Moore, D J Munday, A Parker, $S F$ Takach, TO White

DUBNA - V D Kalagin, A M Kalinin, M N Kapishin, V D Kekelidze, P Khristov, D A Kirillov, I A Kojevnikov, Y K Potrebenikov

EDINBURGH U - I Bertolotto, O Boyle, B Hay, A Main, N McKay, G Oakland, K J Peach, E Veitch, L L J Vick, A Walker

FERRARA U \& INFN, FERRARA - D Bettoni, R Calabrese, P Dalpiaz, J Duclos, P Ferretti Dalpiaz, P Frabetti, A Gianoli, V Guidi, E Luppi, F Petrucci, L Piemontese, F Rossi, M Savrie

FLORENCE U \& INFN, FLORENCE - A Bizetti, M Calvetti

( $\checkmark$ Spokesperson), M Lenti, A Michetti

MAINZ U, INST PHYS - H-G Becker, T Beier, H Bluemer, B Bockholt, C Ebersberger, A Kalter, K Kleinknecht, HC Knoeppler, U Koch, L Koepke, S Luitz, B Renk, J Scheidt, J Schmidt, V Schoenharting, J Staeck, R Wilhelm, A Winhardt, $M$ Wittgen

VIENNA, OAW - H Dibon, M Jeitler, M Markytan, I Mikulec, G Neuhofer, M Pernicka, A Taurok, C E Wulz

ORSAY, LAL -- J-N Albert, E Auge, G Barrand, J-E Campagne, J-C Chollet, $\mathrm{C}$ de la Taille, L Fayard, L Iconomidou-Fayard, G Martin-Chassard, A Schaffer, G Unal

PERUGIA U \& INFN, PERUGIA - G Anzivino, F Bordacchini, $P$ Cenci, A Del Rosso, P Lubrano, M Pepe, M Punturo, C Talamonti

PISA U \& INFN, PISA - A Bast, L Bertanza, A Bigi, P Calafiura, R Carosi, C Cerri, F Costantini, R Fantechi, F Fidecaro, B Gorini, F Laico, I Mannelli, V Marzulli, G M Pierazzini, F Raffaelli

TURIN U - C Biino, A Ceccuci, R Cester, P Maas, F Marchetto. E Menichetti, R Mussa, S Palestini, N Pastrone

SIEGEN U - I Augustin, M Bender, G Gillessen, M Holder, W Otto, M Roschangar, C Schmitz, B Schofer, R Werthenbach. $S$ Winkler

WARSAW U - A Chlopik, Z Guzik, J Nassalski, E Rondio, M Szleper, W Wislicki

Accelerator CERN-SPS Detector Calorimeter, Spectrometer

Particles studied $K_{S}, K_{L}, \Lambda$

Brief description The goal is to measure $\operatorname{Re}\left(\epsilon^{\prime} / \epsilon\right)$ with an accuracy of $2 \times 10^{-4}$. The experiment uses the $450 \mathrm{GeV} / c$ SPS proton beam to produce two nearly collinear $K_{S}$ and $K_{L}$ beams concurrently. Kaon decays are distinguished by tagging the protons generating the $K_{S}$ component. The detector is optimized for the detection of $\pi^{+} \pi^{-}$and $\pi^{0} \pi^{0}$ final states from neutral kaons with momenta between 70 and $170 \mathrm{GeV} / c$. Charged decays are measured in a magnetic spectrometer with a central dipole magnet and two sets of large and highprecision drift chambers on each side. Neutral decays are recorded in a homogeneous liquid krypton calorimeter designed for high rate capability, good energy and space resolution, and sub-nanosecond time resolution. This configuration permits collecting all four modes concurrently with minimal systematic error. Other components of the apparatus include a proton tagger, a hadron calorimeter, and counters to veto muons and particles outside the detector acceptance. The first charged kaon test data have been collected during 1995 . The installation is being completed with the positioning of the LKr calorimeter (Summer 96). First data taking is expected in early 1997.

Journal papers NIM A316 (1992) 1, NIM A323 (1992) 393, NIM A344 (1994) 487, NIM A344 (1994) 507, NIM A360 (1995) 224, NIM A361 (1995) 466, NIM A365 (1995) 268, NIM ^367 (1995) 263. NIM A370 (1996) 413, and NIM A373 (1996) 213.

Related experiments CERN-PS-195, FNAL-832

E-mail contact mario.calvetti@cern.ch

WWW Home-page http://wwwl.cern.ch/NA48/Welcome.html

CERN-NA-050

(Proposed Nov 1991, Approved Feb 1992, Began data-taking Nov 1994, In progress)

STUDY OF MUON PAIRS AND VECTOR MESONS PRODUCED IN HIGH ENERGY Pb Pb INTERACTIONS

NA50 COLLABORATION 


\section{SUMMARIES OF CERN EXPERIMENTS}

ANNECY - C Baylin, A Bussiere, V Capony, R Kossakowski BUCHAREST, IAP - C Alexa, V Boldea, S Constantinescu, $S$ Dita

CAGLIARI U \& INFN, CAGLIARI - M Ceru, C Cicalo, A De Falco, P Macciotta, A Masoni, G Puddu, S Serci, $P$ Temnikov, G Usai

CLERMONT-FERRAND U - A Baldit, J Castor, T Chambon,

I Chevrot, A Devaux, B Espagnon, J Fargeix, P Force,

$G$ Landaud, L Luquin, S Mourgues, P Saturnini

CRACOW, INST PHYS NUCL TECYI - W Dabrowski

CERN - C Lourenco, R Shahoian, P Sonderegger

LISBON, LIP - M C Abreu, P Bordalo, S Ramos, S Silva

MOSCOW, INR - Y K Gavrilov, M G Golubeva, M Gotra,

T L Karavicheva, A B Kurepin, N S Topilskaya

ORSAY, IPN - J Astruc, C Gerschel, D Jouan, Y Le Bornec,

$\mathrm{X}$ Tarrago, $\mathrm{N}$ Willis

ECOLE POLYTECHNIQUE - $A$ Borhani, B Chaurand, F Fleuret,

M Gonin, L Kluberg ( $\checkmark$ Spokesperson), P Petiau, A Romana

STRASBOURG, CRN - P Gorodetzky, C Racca

TURIN U \& INFN, TURIN - S Alessandro, $S$ Beole, G Bonazzola,

E Chiavassa, $G$ Dellacasa, $N$ De Marco, M Gallio, P Giubellino,

P Guaita, M Idzik, A Marzari Chiesa, M Masera, A Musso,

A Piccotti, W L Prado da Silva, L Ramello, P Rato Mendes,

L Riccati, E Scomparin, E Vercellin

LYON, IPN - M Bedjidian, F Bellaiche, B Cheynis, O Drapier,

I X Grossiord, A Guichard, $R$ Haroutunian, M Jacquin,

F Malek, J R Pizzi

YEREVAN PHYS INS'T -. A A Grigoryan, H R Gulkanyan,

R S Hakobyan, S S Mehrabyan

Accelerator CERN-SPS Detector Calorimeter, Spectrometer

Reactions

$$
\mathrm{Pb} \text { nucleus } \rightarrow \mu^{+} \mu^{-} \mathrm{X} \quad 160 \mathrm{GeV}\left(\mathrm{T}_{\mathrm{lab}} / \mathrm{N}\right)
$$

Partzclesstudied vmeson

Brief description Studies dimuons produced in $\mathrm{Pb}-\mathrm{Pb}$ collisions at the nucleon-nucleon $E_{c . m}$. of $18 \mathrm{GeV}$. The setup is optimized for a mass range which includes signals probing QGP (Quark and Gluon Plasma), namely $\phi, J / \psi, \psi^{\prime}$, and (unseparated) $\rho$ and $\omega$ vector mesons. It also covers Drell-Yan dimuons which serve as a normalization. The detector is an improved version of the CERN-NA-038 setup, with neutral energy and multiplicity detectors following the target, the Zero Degrees quartz fiber hadron calorimeter (ZDC) embedded in the hadron dump, and a muon spectrometer following the dump. Taking data (June 96).

Related experiments CERN-NA-038

E-mail contact louis.kluberg@cern.ch, peter.sonderegger@cern.ch WWW Home-page http://www.cern.ch/NA50/

\section{CERN-NA-051}

(Proposed Apr 1992, Approved Apr 1992, Began data-taking Jun 1992, Completed data-taking Jul 1992)

\section{DRELL-YAN STUDY OF SEA ISOSPIN SYMMETRY}

\section{NA51 COLLABORATION}

CERN -- P Sonderegger

CLERMONT-FERRAND U - A Baldit, C Barriere, I Castor,

T' Chambon. A Devaux, B Espagnon, J Fargeix, P Force,

G Landaud, P Saturnini, F Vazeille

LISBON, LIP M C Abreı, P Bordalo, R Ferreira, C Lourenco,

$S$ Ramos, S Silva, $J$ Varela

ORSAY, IPN - C Gerschel, D Jouan, X Tarrago

ECOLE POLYTECHNIQUE - B Chaurand, L Kluberg

( $\checkmark$ Spokesperson), A Romana

STRASBOURG, CRN -- P Gorodetzky, D Lazic, R Mazini, C Racca

TURIN U \& INFN, TURIN - B Alessandro, E Chiavassa,

G Dellacasa, M Gallio, P Giubellino, P Guaita, A Marzari-

Chiesa, M Masera, M Monteno, A Musso, L Ramello, L Riccati, E Scomparin. E Vercellin

LYON, IPN - M Bedjidian, D Contardo, E Descroix, O Drapier,

J Y Grossiord, A Guichard, R Haroutunian, F Malek,

R Mandry, J R Pizzi

Accelerator CERN-SPS Detector Spectrometer
Reactions

$$
\begin{array}{lc}
p p \rightarrow \mu^{+} \mu^{-} \mathrm{X} & 450 \mathrm{GeV} / c\left(\mathrm{P}_{\mathrm{lab}}\right) \\
p \text { deut } \rightarrow \mu^{+} \mu^{-} \mathrm{X} & "
\end{array}
$$

Brief description The purpose of the experiment is to study

the isospin symmetry in the light-quark sea of the proton. Its violation is one possible explanation of recent unexpected muon deep inelastic scattering experimental results which disagree with the Gottfried sum rule. The experiment makes use of the large acceptance muon spectrometer used previously by CERNNA-010 and CERN-NA-038. It detects muon pairs produced by the Dreil-" Yan mechanism in $p p$ and $p d$ reactions. A beam of $450 \mathrm{GeV} / c$ protons impinges on alternating liquid hydrogen and deuterium targets. The aim is to measure the cross-section ratio at dimuon masses above $4 \mathrm{GeV} / \mathrm{c}^{2}$, which is a sensitive probe of the rclative content of light antiquarks $\bar{u}$ and $\vec{d}$ in the proton sea.

Journal papers PL B332 (1994) 244.

Related experiments FNAL-288, FNAL-711

E-mail contact louis.kluberg@cern.ch

WWW Home-page http://yoinfo.in $2 \mathrm{p} 3 . \mathrm{fr} /$ eiexp/na51.html

\section{CERN-PS-185-2}

(Proposed Jan 1992, Approved Nov 1992, Began data-taking Jul 1994, Completed data-taking Jun 1995)

HIGH PRECISION MEASUREMENT OF $\bar{p} p \rightarrow \bar{\Lambda} \Lambda$ CROSS-SECTIONS IN THE MASS REGION AROUND $2232 \mathrm{MeV} / \mathrm{c}^{2}$

CERN - N Hamann, (deceased)

CARNEGIE MELLON U - G Franklin, R McCrady, C A Meyer,

B Quinn, R Schumacher

ERLANGEN U - H Dennert, W Eyrich, J Hauffe, F Stinzing

FREIBURG U - D Afzali, J Franz, J Rauch, E Rocssle, M Ruh.

D Ruschmeier, H Schmitt, R Todenhagen, T Walter, H Wirth

JULICH, FORSCHUNGSZENTRUM - R Broeders, R Gcyer,

K Kilian ( $\checkmark$ Spokesperson), W Oelert, K Roehrich

( $\checkmark$ Spokesperson), K Sachs, $\Upsilon$ Sefzick

LOS ALAMOS - P D Barnes

ILLINOIS U, URBANA $\cdots$ R A Eisenstein, P Harris, D Hertzog, $T$ Jones, $\mathrm{R}$ Tayloe

NEW MEXICO U - H Fischer

UPPSALA U - T Johansson, E Traneus

Accelerator CERN-LEAR Detector Wire chamber

Reactions

$$
\bar{p} p \rightarrow \bar{\Lambda} \Lambda \quad 1.435-1.450 \mathrm{GeV} / c\left(\mathrm{P}_{\mathrm{lab}}\right)
$$

Brief description The aim of the experiment is to verify a possible structure in the excitation function of the reaction $\bar{p} p \rightarrow \bar{\Lambda} \Lambda$ indicated by the recent threshold data of the CERN-PS-185 collaboration at an invariant mass of about $2232 \mathrm{MeV} / \mathrm{c}^{2}$. Studies the total and differential cross-sections as well as polarizations and spin correlations, within a fcw $\mathrm{MeV}$ around the reaction threshold. Uses an upgraded version of the PS-185 detector. The full $\bar{\Lambda} \Lambda$ kinematics is reconstructed from tracks in a $30 \mathrm{~cm}$ long stack of MWPC's and drift chambers. The production vertex is reconstructed by making use of four planes of $\mu$-strip counters upstream the $\mathrm{CH}_{2}$ sandwich target. Hyperon and antihyperon are distinguished by means of a magnetic solenoid with threc drift chambers inside. The setup provides a large acceptance, a high efficiency, and a low annihilation background.

Related experments CERN-PS-185, CERN-PS-185-3

E-mail contact klaus.rohrich@cern.ch

WWW Home-page

http://hpfr02.physik.uni-freiburg.de/ps185/ps185.html

\section{CERN-PS-195}

(Proposed Jan 1985, Approved Sep 1985, Began data-taking 1991, (n progress)

TESTS OF CP VIOLATION WITH $\vec{K}^{0}$ AND $K^{0}$ AT LEAR 


\section{SUMMARIES OF CERN EXPERIMENTS}

CPLEAR COLLABORATION

ATHENS U - A Angelopoulos, A Apostolakis, E Rozaki, L Sakelliou, K Sarigiannis

BASEL U - P Kokkas, F Leimgruber, P Pavlopoulos ( $\sqrt{ }$ Spokesperson), G Polivka, R Rickenbach, T Schietinger, L Tauscher, S Vlachos

BOSTON U - M Chertok, D Francis, J P Miller, B L Roberts, D Zimmerman

SACLAY - G Chardin, M Dejardin, J Derre, D Garreta, C Guyot, C Kochowski, G Marel, P Schune, A Soares, C Yeche

CERN - P Bloch, M Fidecaro, T Ruf, A Schopper, C Touramanis COIMBRA U .. J Carvalho, $R$ Ferreira-Marques, E Machado,

$\mathrm{J}$ Pinto da Cunha, E Von Beveren

DELFT UNIV TECH - R W Hollander, R Kreuger,

C W E Van Eijk

FRIBOURG U - F Blanc, L Faravel, L A Schaller

IOANNINA U - I Evangelou, N Manthos, F Triantis

LIVERPOOL U - A Benelli, M Caroll, E Cawley, A Cody,

J R Fry, E Gabathuler, R Gamet, A Haselden, P I Hayman LJUBLJANA U - A Filipcic, I Mandic, M Mikuz, D Zavrtanik ORSAY, CSNSM - C Thibault

PSI, VILLIGEN - P R Kettle, T Nakada, O Wigger

ROYAL INST TECH, STOCKHOLM - P Carison, M Danielsson,

A Go, K Jon-And

THESSALONIKI U - S Charalambous, S Dedoussis,

$C$ Eleftheriadis, $A$ Liolios, I Papadopoulos

MARSEILLE, CPPM - E Aslanides, V Bertin, A Ealet, F HenryCouannicr, E Hubert, $R$ Le Gac, F Montanet, F Touchard

ZURICH, ETH - O Behnke, W Fetscher, H J Gerber, B Pagels,

M Schaefer, P Weber, M Wolter

Accelerator CERN-LEAR Detector Spectrometer, Calorimeter

\section{Reactions}

$$
\begin{array}{lc}
\bar{p} p \rightarrow K^{0} \mathrm{X} & 0 \mathrm{GeV} / c \\
\bar{p} p \rightarrow \bar{K}^{0} \mathrm{X} & "
\end{array}
$$

\section{Particles studied $\quad K^{0}, \bar{K}^{0}$}

Brief descraption Measures time-dependent $K^{0}-\overline{K^{0}}$ decay rate asymmetries for nonleptonic and semileptonic decays. They are sensitive to $C P$ and $T$ violation in different and complementary ways, and also provide sensitive tests of $C P T$. A beam of $10^{6} \bar{p} /$ sec provided by LEAR is brought to rest in a hydrogen gas target, producing $K^{0}$ and $\bar{K}^{0}$ through the reaction $\bar{p} p$ $\rightarrow K^{ \pm}{ }_{\pi} K^{0}\left(\bar{K}^{0}\right)$. Decays of the $K^{0}$ and $\bar{K}^{0}$ are recorded under the same operating conditions, inside a magnetic field and using tracking chambers and a gas sampling electromagnetic calorimeter. The strangeness of the neutral kaon is tagged by detecting the sign of accompanying charged kaon identified by Cerenkovs and scintillators. Hardware processors are used to reconstruct and select different decay topologies on-line in less than 25 microseconds. Taking data (July 96).

Journal popers NIM A279 (1989) 285, NIM A279 (3989) 305,

NIM A279 (1989) 317, NIM A283 (1989) 484, NC 102A (1989) 127, NIM A297 (1990) 126, NIM A301 (1991) 424, NP (PROC SUPPL) 24A (1991) 45, PL B267 (1991) 154, NIM A311 (1992) 78, NIM A321 (1992) 458, NIM A323 (1992) 511, NP (PROC SUPPL) 27 (1992) 285, PW 3 (1992) 40, PL B286 (1992) 180, SJNP 55 (1992) 840, NIM A333 (1993) 93, NIM A333 (1993) 513, NP (PROC SUPPL) 31 (1993) 196, NP A558 (1993) 43c, NP A558 (1993) 437c, NP A558 (1993) 449c, PAN 57 (1994) 1772, ZPHX C63 (1994) 541, PL B363 (1995) 237, PL B363 (1995) 243, PL B364 (1995) 239, ZPHY C65 (1995) 199, NIM A368 (1996) 666. PL B369 (1996) 367, PL B370 (1996) 167. PI, B374 (1996) 313, and ZPYY C70 (1996) 211

Related experiments CERN-NA-048, FNAL-621, FNAL-832

E-mail contact noulis.pavlopoulos@cern.ch

WWW Home-page http://wwwl.cern.ch/cplear/Welcome.html

\section{CERN-PS- 196}

(Proposed Mar 1985, Approved Nov 1985, In progress)

PRECISION COMPARISON OF $\bar{p}$ AND $p$ MASSES IN A PENNING TRAP
HARVARD U - G Gabrielse ( $\checkmark$ Spokesperson), D Phillips

MAINZ U, INST PHYS - K Heimann, H Kalinowsky

SEOUL NATIONAL U - W Jhe

Accelerator CERN-LEAR Detector Other

Particles studied $\bar{p}$

Brief description Compares $p$ and $\bar{p}$ masses to an accuracy of one part in $10^{10}$ witlin a small volume of an ion trap, and develops trapping and cooling techniques to allow the production and study of low energy antiprotons and antihydrogen. Antiprotons have been trapped below $3 \mathrm{keV}$. Electron cooling from $\mathrm{keV}$ to $<10^{-3} \mathrm{eV}$ has been observed in the trap. Also measures the antiproton storage lifetime. First attempt to make cold antihydrogen is scheduled for late 1996. Taking data through Dec 96.

Journal papers PRL 57 (1986) 2504, RSI 58 (1987) 2197, PL

A129 (1988) 38, PRL 63 (1989) 1360, PRL 65 (1990) 1317,

PRL 74 (1995) 3544, PRL 75 (1995) 806, and PRL 77 (1996) (in press).

E-mail contact gabrielse@hussle.harvard.edu

\section{CERN-PS-197}

(Proposed Oct 1985, Approved Apr 1986, Began data-taking 1989, In progress)

\section{THE CRYSTAL BARREL: MESON SPECTROSCOPY AT LEAR WITH A $4 \pi$ DETECTOR}

\section{CRYSTAL BARREL COLLABORATION}

RUHR U, BOCHUM - K Beuchert, T Degener, H Koch, M Kunze, $J$ Luedemann, H Matthacy, K Peters, H Stoeck

BONN U - B Barnett, R Hackmann, M Herz, H Kalinowsky,

B Kaltcyer, E Klempt, S Resag, C Strassburger, U Thoma

BUDAPEST, CRIP .. P Hidas

CERN - M Doser, J Kisiel, R Landua, L Montanet, R Ouared, U Wiedner

CARNEGIE MELLON U - A Berdoz, R McCrady, C A Meyer

HAMBURG U - J Meier, P Schmidt, R Seibert, U Strohbusch

KARLSRUIIE U - S Bischof, $P$ Bluem, D Engelhardt,

C Holtzhaussen, $M$ 'Iischhaeuser

LBL - D Armstrong, T Case, $\mathrm{K} M$ Crowe, $F$ H Heinsius, P Kammel, M Lakata

QUEEN MARY - WESTFIELD COLL - D V Bugg, I Scott, B Zou

MUNICH U - K Braune, O Cramer, N Djaoshvili, W Duennweber, M A Faessler ( $\checkmark$ Spokesperson), N P Hessey, D Jamnik, C Regenfus, W Roethel, C Voelcker, D Walther, C Zupancic NORTHWESTERN U - B Kaemmle, K Seth

PARIS, CURIE UNIV VI \& PARIS, UNIV VII, LPNIIE M Benayoun

RUTIERFORD - C A Baker, C J Batty, C Pinder

STRASBOURG, CRN - M Suffert

UCLA $\cdots R$ P Haddock

ZURICH U - C Amslcr, P Giaritta, F Ould-Saada, S Spanier,

$S$ von Dombrowski

Accelerator CERN-LEAR Detector CRYSTAL-BARREL

Reactions
$\bar{p} p \rightarrow$ annihil
$0.2000 \mathrm{MeV} / \mathrm{c}$

$\bar{p} n \rightarrow$ annihil

Particles studied glueball, meson

Brief description The spectrometer is designed to provido complete and precise information on nearly every final state produced in $\bar{p} p$ and $\ddot{p} d$ annihilations at low energy and to collect high statistics data samples. The physics goal is to identify all light mesons in the mass range from 0.14 to $2.3 \mathrm{GeV} / \mathrm{c}^{2}$, to determine their properties and the annihilation dynamics, and to search for the glueball and hybrid degrees of freedom The apparatus consists of a barrel shaped electromagnctic calorimeter with $1380 \mathrm{CsI}(\mathrm{Tl})$ crystals read out by photodiodes. a cylindrical jet drift chamber, a silicon $\mu$-strip vertex detector, and silicon hodoscopes. The whole detector is embedded in a solenoidal magnet with field strength up to $1.5 \mathrm{~T}$. Uses liquid and gaseous $\mathrm{H}_{2}$ and $\mathrm{D}_{2}$ targets. Scheduled to rum till the end of 1996. 


\section{SUMMARIES OF CERN EXPERIMENTS}

Journal papers NP (PROC SUPPL) 8 (1989) 65, PL B260

(1991) 249, NP A527 (1991) 491c, IEEE TNS 39 (1992) 826, NIM A321 (1992) 69, PL B291 (1992) 347, PL B294 (1992) 451, PL B297 (1992) 214, SJNP 55 (1992) 767, PL B311 (1993) 362, PL B311 (1993) 371, PL B319 (1993) 373, ZPHY C58 (1993) 175, PAN 57 (1994) 1711, PL B322 (1994) 431, PL B323 (1994) 233, PL B327 (1994) 425, PL B333 (1994) 271, PL B333 (1994) 277 , PL B340 (1994) 259, PAN 57 (1994) $1465=$ YF 57 (1994) 1542, NC 107A (1994) 1815, ZPHY A351 (1994) 325, PL B342 (1995) 433, PL B346 (1995) 203, PL B346 (1995) 363, PL B352 (1995) 187, PL B353 (1995) 571, PL B355 (1995) 425, and PL B358 (1995) 389.

Related experiments CERN-PS-201

E-mail contact martin.faessler@cern.ch

WWW Home-page http://www.phys.cmu.edu/cb/cb.html

\section{CERN-PS-201}

(Proposed Jan 1986, Approved Sep 1986, Began data-taking Aug 1990 , In progress)

STUDY OF ANTINUCLEON ANNIHILATIONS AT LEAR WITH OBELIX, A LARGE-ACCEPTANCE AND HIGH RESOLUTION DETECTOR BASED ON THE OPEN AXIAL FIELD SPECTROMETER

OBELIX COLLABORATION

BOLOGNA U \& INFN, BOLOGNA - A Bertin, M Bruschi, M Capponi, I D'Antone, S De Castro, A Ferretti, D Galli, B Giacobbe, U Marconi, I Massa, M Piccinini, M Poli,

N Semprini-Cesari, R Spighi, S Vecchi, A Vezzani, F Vigotti, $M$ Villa, A Vitale, A Zoccoli

BRESCIA U \& INFN, BRESCIA - G Belli, M Corradini, A Donzella, E Lodi Rizzini, L Venturelli, A Zenoni

CAGLIARI U \& INFN, CAGLIARI - A Adamo, C Cicalo, A Lai, A Masoni, L Musa, G Puddu, S Serci, P Temnikov, G L Usai

DUBNA - O Y Denisov, O E Gorchakov, V P Nomokonov,

S N Prakhov, A M Rozhdestvensky, M G Sapozhnikov,

$\mathrm{V}$ I 'rretyak

FRASCATI - P Gianotti, C Guaraldo, A Lanaro, V Lucherini, F Nichitiu, C Petrascu, A Rosca

LEGNARO - V G Ableev, C Cavion, U Gastaldi, L Lombardi, $G$ Maron, R A Ricci, L Vannucci, G Vedovato

PADUA U \& INFN, PADUA - A Andrighetto, M Morando

PAVIA U \& INFN, PAVIA - G Bendiscioli, V Filippini,

A Fontana, C Marciano, P Montagna, A Rotondi

( $\sqrt{ }$ Spokesperson), $A$ Saino, $P$ Salvini

TURIN POLYTECHNIC \& INFN, TURIN - M Agnello, F Iazzi, B Mincti

TURIN U \& INFN, TURIN - F Balestra, G C Bonazzola, E Botta, 'I Bressani, M P Bussa, L Busso, D Calvo, P Cerello, $S$ Costa, D D'Isep, L Fava, A Feliciello, L Ferrero, A Filippi, R Garfagnini, A Grasso, A Maggiora, S Marcello, D Panzieri,

D Parena, E Rossetto, F Tosello, G Zosi

TRIESTE U, IST FIS \& INFN, TRIESTE - G Margagliotti,

G Pauli, S Tessaro

UDINE U \& INFN, UDINE - L Santi

Accelerator CERN-LEAR Detector Spectrometer

Reactions

$\bar{p} p \ldots$ annihil

$\bar{p}$ deut $\rightarrow$ annihil

$\bar{p}$ nucleus $\rightarrow$ annihil

$\bar{n} p-$, annihil

$\bar{n}$ nucleus $\rightarrow$ annitii

$$
\begin{gathered}
0-0.2 \mathrm{GeV} / c\left(P_{\mathrm{lab}}\right) \\
" \\
" \\
0-0.4 \mathrm{GeV} / c\left(P_{\mathrm{lab}}\right)
\end{gathered}
$$

Partzcles studied mesons

Brief description Studies (1) spectroscopy of $q \bar{q}$, exotic, glueball,

and hybrid mesons, (2) dynamics of $N \bar{N}$ intcractions, (3) atomic physics with $\bar{p}$ 's, and (4) $\bar{p}$ annihilations onto more than one nucleon. A Spiral Projection Chamber (SPC) is used as vertex detector. The magnet and the jet drift chamber of the Open Axial Field Spcctrometer (AFS) are used for charged particles momentum and $\mathrm{d} E / \mathrm{d} x$ measurements. Two concentric arrays of plastic scintillators identify and trigger on charged kaons. Gamma showers are reconstructed in three dimensions with gas sampling calorimeter moduli. Taking data (July 96). Scheduled to complete the data taking by the end of 1996 .

Journal papers IEEE TNS 38 (1991) 331, IEEE TNS 38 (1991) 337, IEEE TNS 38 (1991) 393, NIM A306 (1991) 305, PL B256 (1991) 349, NIM A323 (1992) 523, PL B284 (1992) 448, PL B285 (1992) 15, PL B287 (1992) 368, SJNP 55 (1992) 806, NIM A325 (1993) 417, NIM A334 (1993) 391, NP A553 (1993) 651c, NP A558 (1993) 13c, NP A558 (1993) 137c, NP A558 (1993) $369 c$, NP A558 (1993) 665c, NP A562 (1993) 617, PR A47 (1993) 4517, PL B329 (1994) 407, PL B334 (1994) 237, PL B337 (1994) 226, PAN 57 (1994) 1614 = YF 57 (1994) 1684, PAN 57 (1994) $1716=\mathrm{YF} 57$ (1994) 1787, PAN 57 (1994) $1745=\mathrm{YF}$ 57 (1994) 1816, NC A107 (1994) 943, NC A107 (1994) 1325, NC A 107 (1994) 2837, NP A569 (1994) 761, NIM A356 (1995) 270, PL B361 (1995) 187, PRL 74 (1995) 371, NP A585 (1995) 577 NP A594 (1995) 375, and PL B369 (1996) 77.

Related experiments CERN-PS-195, CERN-PS-197, CERN-PS202, BNL-852, FNAL-760

E-mail contact alberto.rotondi@cern.ch, rotondi@vxcern.cern.ch

\section{CERN-PS-202}

(Proposed 1986, Approved Feb 1987, Began data-taking Jul 1991, Completed data-taking Sep 1994)

JETSET: PHYSICS AT LEAR WITH AN INTERNAL GAS JET TARGET AND AN ADVANCED GENERAL PURPOSE DETECTOR

PS202 COLLABORATION

BARI U \& INFN, BARI - C Evangelista, A Palano

CERN - D Drijard, M Ferro-Luzzi. R Jones, B Mouellic,

$J$ M Perreau, M J Price

ERLANGEN U - W Eyrich, R Geyer, S Pomp, F Stinzing

FREIBURG U - H Fischer, J Franz, E Roessle, H Schmitt, $M$ Tscheulin, $\mathrm{H}$ J Urban, $H$ Wirth

GENOA U \& INFN, GENOA - A Buzzo, M Loveterc, M Macri

( $\checkmark$ Spokesperson), M Marinelli, S Passaggio, M G Pia, A Pozzo, E Robutti, A Santroni

ILLINOIS U, URBANA - $P$ Debevec, $R$ A Eisenstein, $P$ Harris,

D Hertzog, S Hughes, P Reimer, J Ritter

KERNFORSCHUNGSANLAGE, JULICH - K Kilian, W Oelert,

K Roehrich, M Rook, O Steinkamp

OSLO U - H Korsmo

UPPSALA U - T Johansson

Accelerator CERN-LEAR Detector JETSET

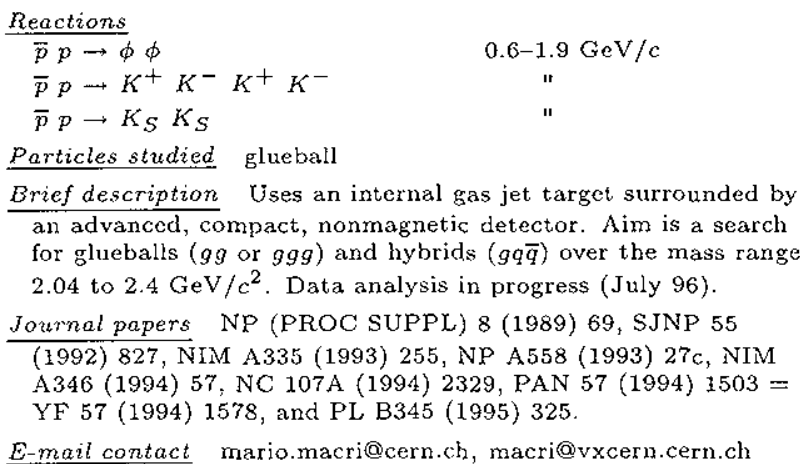

\section{CERN-UA-004-2}

(Approved Jul 1990, Completed data-taking)

A PRECISE MEASUREMENT OF THE REAL PART OF THE ELASTIC SCATTERING AMPLITUDE AT THE $\mathrm{S} \bar{p} p \mathrm{~S}$

GENOA U \& INFN, GENOA - M Bozzo, G Sette, M Zito ECOLE POLYTECHNIQUE - C Augier, D Bernard, J Bourotte, M Haguenauer (Spokesperson) 


\section{SUMMARIES OF CERN EXPERIMENTS}

PRAGUE, INST PHYS - V Kundrat, S Nemecek, M Novak, M Smizanska

ROME U, TORVERGATA \& INFN, ROME - R Cardarelli, $L$ Cerrito, G Matthiae, $F$ Natali

VALENCIA U - F Alted, $\mathrm{R}$ Cases, E Sanchis, J Velasco Accelerator CERN-PBAR/P Detector. Wire chamber Reactions

$$
\bar{p} p \rightarrow \bar{p} p \quad 630 \mathrm{GeV}\left(\mathrm{E}_{\mathrm{cm}}\right)
$$

Brief description Measures the total cross-section and the ratio $\rho$ of the real to the imaginary part of the forward elastic scattering amplitude. The setup is composed of two pairs of Roman Pots placed symmetrically at $45 \mathrm{~m}$ from the crossing point. The horizontal scattering angle is measured by drift chambers and the vertical coordinate is obtained by using a hodoscope. Data analysis in progress (June 96).

Journal papers PL B315 (1993) 503, PL B316 (1993) 448, and PL B344 (1995) 451

Related experiments CERN-UA-004

E-mail contact haguen@frcpn11.in2p3.fr

\section{CERN-WA-079}

(Proposed Apr 1983, Approved Jun 1983, Began data-taking Aug 1986, Completed data-taking Aug 1991)

STUDY OF NEUTRINO-ELECTRON SCATTERING AT THE SPS

CHARM-II COLLABORATION

BRUSSELS U, IMHE - P Vilain, G Wilquet

CERN - R Beyer, W Flegel, H Grote, T Mouthuy, H Overas,

J Panman, $A$ Rozanov, $K$ Winter ( $\checkmark$ Spokesperson), G Zacek, $\checkmark$ Zacek

HAMBURG U - F W Busser, C Foos, L Gerland, T Layda,

F Niebergall, G Radel, P Stahelin, T Voss

LOUVAIN U - D Favart, G Gregoire, E Knoops, V Lemaitre

MOSCOW, ITEP - P Gorbunov, E A Grigoriev, V D Khovansky, A Maslennikov

MUNICH U, EXP PHYS - W Lippich, A Nathaniel, A Staude, J Vogt

NAPLES U, IFS \& INFN, NAPLES - A G Cocco, A Ereditato, G Fiorillo, F Marchetti-Stasi, V Palladino, P Strolin

INFN, ROME - A Capone, D De Pedis, U Dore, A Frenkel-

Rambaldi, P F Loverre, D Macina, G Piredda, R Santacesaria

FERRARA U \& INFN, FERRARA - E Di Capua, S Ricciardi, B Saitta

MIDDLE EAST TECH U, ANKARA - B Akkus, E Arik, M SerinZeyrek, $R$ Sever, $P$ Tolun

BERLIN-ZEUTIEN ADW - K Hiller, R Nahnhauer, H E Roloff Accelerator CERN-SPS Detector CHARM-II

Reactions

$$
\begin{array}{cc}
\nu_{\mu} e^{-} & 5-100 \mathrm{GeV} / c \\
\bar{\nu}_{\mu} e^{-} & " 1
\end{array}
$$

Brief description Aims at determining the electroweak mixing angle $\theta_{W}$ and the ratio $g_{A} / g_{V}$ from the ratio of $\nu e^{-}$and $\bar{\nu} e^{-}$scattering cross-sections. The obtained values are to be compared to those determined with $10^{6}$ times larger $Q^{2}$ at LEP. The CHARM-II neutrino detector consists of a massive, finegrained, and low-density electronic calorimeter, followed by a muon spectrometer made of magnetized iron, with scintillators and drift chambers as active elements. The measurements are performed in the horn-focused wide band neutrino beam.

Journal papers NIM A252 (1986) 443, NIM A260 (1987) 368,

NIM A263 (1988) 109, PL B213 (1988) 554, NIM A277 (1989) 83, NIM A277 (1989) 170, NIM A278 (1989) 670, PL B231 (1989) 317, PL B232 (1989) 539, PL B245 (1990) 271. PL B247 (1990) 131, NP (PROC SUPPL) 19 (1991) 306, PL B259 (1991) 499, PL B281 (1992) 159, PL B302 (1993) 351, PL B309 (1993) 463, PL B313 (1993) 267, PL B320 (1993) 203, PL B332 (1994) 465, PL B335 (1994) 246, ZPHY C64 (1994) 539, PL B343 (1995) 453, PL B345 (1995) 115, and PL B364 (1996) 121.

E-mail contact klaus.winter@cern.ch

\section{CERN-WA-085}

(Proposed Oct 1984, Mar 1987, Approved Apr 1987, Began datataking Oct 1987, Completed data-taking Sep 1991)

STUDY OF HIGH ENERGY NUCLEUS-NUCLEUS INTERACTIONS USING THE $\Omega^{\prime}$ SPECTROMETER EQUIPPED WITH A MULTIPARTICLE HIGH $p_{\perp}$ DETECTOR

WA85 COLLABORATION

ATHENS U - $S$ Abatzis, $G$ Vassiliadis

BARI U - N Di Bari, D Elia, R Fini, B Ghidini, A Jacholkowski,

V Lenti, R A Loconsole, V Manzari, F Navach

BERGEN U - H Helstrup

BIRMINGHAM U - R Barnes, A C Bayes, J N Carney,

J P Davies, D Evans ( $\checkmark$ Spokesperson), J B Kinson, A Kirk,

$M$ Venables, $O$ Villalobos-Baillie, $M F$ Votruba

CERN - A Andrighetto, F Antinori, W Beusch, J P Dufey,

B R French, A K Holme, K Knudson, J C Lassalle, E Quercigh,

L Rossi, K Safarik

MADRID, CIEMAT - B de la Cruz

COLLEGE DE FRANCE - M Benayoun, J Kahane, P Leruste,

J L Narjoux, M Sene, R Sene, A Volte

OSLO U - J A Lien, $T$ Storas

Accelerator CERN-SPS Detector OMEGA-PRIME

Reactions

$p \mathrm{Wt}$

$200 \mathrm{GeV}\left(\mathrm{T}_{\mathrm{lab}}\right)$

${ }^{32} \mathrm{~S} \mathrm{Wt}$

$200 \mathrm{GeV}\left(\mathrm{T}_{\mathrm{lab}} / \mathrm{N}\right)$

Particles studied $K^{0}, \Lambda, \bar{\Lambda}, \Xi^{-}, \Xi^{+}, \Omega^{-}, \bar{\Omega}+$

Brief description An exploratory experiment to look for new physics, and particularly for evidence of a quark-gluon plasma, through an increase in strange particle and antiparticle production. Some of the goals are a study of $\Xi$ and anti- $\Xi$ production, and the full reconstruction of $\Omega$ and anti- $\Omega$

hyperons. Also compares the strange and multistrange particle (anti-particle) productions in $p \mathrm{~W}$ and $\mathrm{S} \mathrm{W}$ interactions. Data analysis in progress (July 96 ).

Journal papers NP A498 (1989) 369c, PL B244 (1990) 130, NP (PROC SUPPL) 16 (1990) 409, PL B259 (1991) 508, PL B270 (1991) 123, NP A525 (1991) 441c, NP A525 (1991) 445c, NP A544 (1992) 321c, PL B316 (1993) 615, NP A566 (1994) 225c, NP A566 (1994) 491c, PL B347 (1995) 158, PL B355 (1995) 401. PL B359 (1995) 382, and NP A590 (1995) 307c.

Related experiments CERN-WA-094, CERN-WA-097

E-mail contact devans@ion.cern.ch, david.evans@cern.ch

\section{CERN-WA-089}

(Proposed Aug 1987, Aug 1987, Approved Feb 1988, Began datataking 1990, Completed data-taking Sep 1994)

\section{NEW HYPERON BEAM EXPERIMENT AT THE CERN-SPS USING THE OMEGA FACILITY}

BRISTOL U - D Newbold, V Smith

CERN .- W Beusch, W Klempt

GENOA U \& INFN, GENOA - D Barberis, L Rossi

GRENOBLE U -. C Berat, M Buenerd. F Charignon, J Chauvin.

A Fournier, $P$ Martin, M Rey-Campagnolle, $E$ Vesin

HEIDELBERG, MAX PLANCK INST -- E Albertson,

$M$ Beck, S Brons, W Brueckner, C Buescher, U Dersch,

F Dropmann. S G Gerassimov, $M$ Godbersen, 'T Haller,

M Heidrich, K Koenigsmann, I Konorov, D Maier, S Masciocci,

R Michaels, C Newsom, S Paul (Spokesperson), B Povh, Z Ren

L Schmitt, A Trombini, K Vorwalter, $\mathrm{R}$ Werding, E Wittmann, M Zizelsberger

HEIDELBERG $U$ - M Boss, P Lennert, K Martens, H Rieseberg,

H W Siebert, A Simon, O Thilmann, G Waelder

MAINZ U, INST KERNPHYS - E Chudakov, U Mueller,

G Rosner, H Rudolph, B Volkemer, 'I' Walcher

LEBEDEV INST - M I Adamovich, Y A Alexandrov,

$M V$ Zavertyaev

RUTGERS U . R Ransome

Accelerator CERN-SPS Detector OMEGA 


\section{SUMMARIES OF CERN EXPERIMENTS}

$\begin{array}{lc}\frac{\text { Reactions }}{\Sigma^{-} \mathrm{Cu}} & 330 \mathrm{GeV} / \mathrm{c}\left(\mathrm{P}_{\mathrm{lab}}\right) \\ \Sigma^{-} \mathrm{C} & " \\ \Xi^{-} \mathrm{Cu} & 270 \mathrm{GeV} / \mathrm{c}\left(\mathrm{P}_{\mathrm{lab}}\right) \\ \Xi^{-} \mathrm{C} & " \\ \Omega^{-} \mathrm{Cu} & " \\ \Omega^{-} \mathrm{C} & "\end{array}$

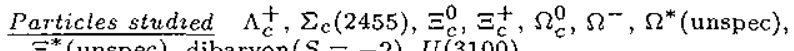
$\Xi^{*}$ (unspec), dibaryon $(S=-2), U(3100)$

Brief description The aims are (1) to study charmed strange baryons, (2) to see if the $U(3100)$ actually exists, (3) to study $\Omega$ decays and $\Xi$ and $\Omega$ resonances, (4) to look for $H$, the doubly strange dibaryon, (5) to measure semileptonic decays of charmed particles, (6) to study hyperon polarization phenomena, and (7) to study $\Sigma^{-} e^{-}$elastic scattering. Uses the upgraded OMEGA facility and a hyperon beam installed at the end of the H1 beamline. Data analysis in progress (July 96).

Journal papers NIM A313 (1992) 203, NIM A313 (1992) 345, NIM A313 (1992) 429, NIM A323 (1992) 373, NIM A338 (1994) 310 , NIM A342 (1994) 364, NIM A343 (1994) 60, NIM A343 (1994) 258, NIM A343 (1994) 279, NIM A348 (1994) 444, NIM A355 (1995) 351, NIM A357 (1995) 274, PL B358 (1995) 151 ZPHY A350 (1995) 379, NIM A371 (1996) 27, and NIM A371 (1996) 192.

Related experments CERN-WA-062

E-mail contact snp@vsnhd1.cern.ch, stephan.paul@cern.ch WWW Home-page http://vsnhd1.cern.ch/

\section{CERN-WA-091}

Proposed Jan 1990, Approved Apr 1990, Began data-taking Jun 1991, Completed data-taking Jun 1994)

SEARCH FOR CENTRALLY PRODUCED NON- $q \bar{q}$ MESONS IN PROTON-PROTON INTERACTIONS AT $450 \mathrm{GeV} / c$ BY USING THE CERN $\Omega$ SPECTROMETER

WA9ג COLLABORATION

ATHENS U - $S$ Abatzis, G Vassiliadis

BARI U \& INFN, BARI - N Di Bari, R Fini, B Ghidini, V Lenti, A Loconsole, V Manzari, $F$ Navach

BIRMINGHAM U - A C Bayes, J N Carney, S Clewer, J P Davies, C, J Dodenhoff, J B Kinson, K Norman, $O$ Villalobos-Baillie, M F Votruba

CERN -. F Antinori, D Barberis, W Beusch, D Evans, B R French, A Jacholkowski, A Kirk ( $\checkmark$ Spokesperson), K Knudson, $\mathrm{J} C$ Lassalle, E Quercigh

DUBNA - Y Kulchitsky, S Maljukov, I Minashvili, V Romanovsky, N IRussakovich, A Semenov, A Soloviev, G Tchlatchidze

COLLEGE DE FRANCE - M Sene, R Sene

Accelerator CERN-SPS Detector OMEGA

Reactions

$p p \rightarrow p p \mathrm{X} \quad 450 \mathrm{GeV} / c$

Particles studied mesons

Brief description A search for new, non- $q \bar{q}$ states in the central region, with at least 10 times the statistics of the CERN-WA076 experiment. Uses a liquid hydrogen target. Data analysis in progress (July 96).

Journal papers PL B324 (1994) 509, NC 107A (1994) 1557, and PL B353 (1995) 589.

Related experiments CERN-WA-076, CERN-WA-102

E-mail contact andrew.kirk@cern.ch

\section{CERN-WA-092}

(Proposed Mar 1990, Approved Jul 1990, Began data-taking Jun 1992, Completed data-taking Sep 1993)

MEASUREMENT OF BEAUTY PARTICLE LIFETIMES AND HADROPRODUCTION CROSS-SECTIONS

BEATRICE COLLABORATION

BOLOGNA U \& INFN, BOLOGNA - A Forino, R Gessaroli, $\mathrm{L}$ Malferrari, P Mazzanti, A Quareni

CERN - F Antinori, W Beusch, J P Dufey, P Farthouat,

B R French. A Kirk, I C Lassalle, M Passaseo, V Ryzhov, G Schuler

DUBNA - S Maljukov, I Minashvili, N Russakovich, A Semenov, A Soloviev

GENOA U \& INFN, GENOA - M Adinolf, D Barberis,

M Dameri, G Darbo, $R$ Hurst, $P$ Martinengo, B Osculati, L Rossi ( $\sqrt{ }$ Spokesperson), C Salvo

MPERIAL COLL - D Barney, J Batten, A Duane, N Hummadi D M Websdale

LEBEDEV INST - M Adamovich, Y Alexandrov, P Nechaeva, M Zavertyaev

PISA U \& INFN, PISA - C Angelini, A Cardini, V Flaminio, C Lazzeroni, C Roda

ROME U \& INFN, ROME - C Bacci, F Ceradini, G Ciapetti,

A Frenkel, $\mathrm{K}$ Harrison, F Lacava, G Martellotti, A Nisati,

D Orestano, G Penso, E Petrolo, L Pontecorvo, M Torelli,

$S$ Veneziano, M Verzocchi, L Zanello

ROME U, TORVERGATA \& INEN, ROME - R Cardarelli,

A Di Ciaccio, $R$ Santonico

SOUTHAMPTON U - J G McEwen

Accelerator CERN-SPS Detector OMEGA-PRIME

Reactions

$\pi^{-}$nucleus $\rightarrow B \bar{B} \times \quad 350 \mathrm{GeV} / c$

Particles studied bottom, charm

Brief description An experimental search for beauty particles produced in fixed target hadronic interactions. Uses a high precision Decay Detector and a fast secondary vertex trigger processor. Data analysis in progress (July 96)

Journal papers NIM A315 (1992) 95, NP (PROC SUPPL) 27 (1992) 251, NIM A337 (1994) 225, NIM A351 (1994) 222, NIM A351 (1994) 225, NIM A361 (1995) 506, NIM A368 (1995) 185 , NP (PROC SUPPL) 44 (1995) 435, PL B348 (1995) 256, and PL B353 (1995) 563

Related experiments FNAL-653, FNAL-771

E-mail contact leonardo@vxcern.cern.ch, leonardo.rossi@cern.ch

\section{CERN-WA-094}

Proposed Jan 1991, Approved Apr 1991, Began data-taking Oct 1991, Completed data-taking Nov 1993)

STUDY OF BARYON AND ANTIBARYON SPECTRA IN SULPHUR-SULPHUR INTERACTIONS AT 200 GeV/c PER NUCLEON

WA94 COELABORATION

A'THENS U - $S$ Abatzis, G Vassiliadis

BARI U - D Di Bari, D Elia, R Fini, B Ghidini, A 3acholkowski, $V$ Lenti, R A Loconsole, V Manzari, F Navach

BERGEN U - E Andersen, K Fanebust, H Helstrup, G Lovhoiden. $P$ Sennels, $T$ F Thorsteinsen

BIR.MINGHAM U - J N Carney, J P Davies, D Evans,

J B Kinson ( $\sqrt{ }$ Spokesperson), A Kirk. $\mathrm{K}$ Norman, M Venables, O Villalobos-Baillie, M F Votruba

COMENIUS U .. J Ftacnik, R Lietava

CERN - F Antinori, W Beusch, B R French, A K Holme,

K Knudson, J C Lassalle, M Passaseo, E Quercigh, K Safarik KOSICE, IEF - J Boehm, I Kralik, K Piska, L Sandor, J Urban, $P$ Zavada

LEGNARO - R A Ricci

MADRID, CIEMAT - B de la Cruz, P Ladron de Guevara

PADUA U \& INFN, PADUA - A Andrighetto, N Carrer,

M Morando, F Pellegrini, G Segato 


\section{SUMMARIES OF CERN EXPERIMENTS}

COLLEGE DE FRANCE - M Benayoun, J Kahane, P Leruste,

J L Narjoux, $M$ Sene, $R$ Sene, A Volte

SERPUKHOV - V A Kachanov, A V Singovsky

STRASBOURG, CRN - T Kachelhoffer, M E Michalon-Mentzer, A Michalon

TRIESTE U \& INFN, TRIESTE - A Bravar, A Penzo

Accelerator CERN-SPS Detector OMEGA

Reactions

$$
\begin{array}{ll}
\mathrm{Su} \mathrm{Su} & 200 \mathrm{GeV}\left(\mathrm{I}_{\mathrm{lab}} / \mathrm{N}\right) \\
p \mathrm{Su} & 200 \mathrm{GeV}\left(\mathrm{T}_{\mathrm{lab}}\right)
\end{array}
$$

Particles studied strange

Brief description Extends analysis of CERN-WA-085 from

$\mathrm{S}$ W to $\mathrm{S} \mathrm{S}$ interactions. For the 1992 run the apparatus has been modified to measure charged particle spectra (in particular $p$ and $\vec{p}$ ) with particle identification using an array of silicon microstrip detectors and a newly upgraded ring imaging Cerenkov detector.

Journal papers NP A566 (1994) 499, PL B354 (1995) 178, NP

A590 (1995) 317, and NIM A371 (1996) 22.

Related experiments CERN-WA-085, CERN-WA-097

E-mail contact jbk@hep.ph.bham.ac.uk

\section{CERN-WA-095}

(Proposed Dec 1990, Approved Sep 1991, Began data-taking May 1994, In progress)

A NEW SEARCH FOR $\nu_{\mu} \leftrightarrow \nu_{\tau}$ OSCILLATIONS

\section{CHORUS COLLABORA'TION}

CUKUROVA U - E Eskut, A Kayis, G Onengut

NIKHEF, AMSTERDAM - J Konijn, R G C Oldeman,

$J$ W E Uiterwijk, $R$ van Dantzig, $C$ A F J van der Poel

MIDDLE EAS'T TECH U, ANKARA - A S Ayan, E Pescn,

$M$ Serin-Zeyrek, R Sever, P Tolun, M T Zeyrek

BARI U - N Armenise, F Cassol, M G Catanesi, M T Muciaccia,

E Radicioni, S Simone

HUMBOLDT U, BERLIN -- P Lendermann, A Meyer-Sievers,

$\Upsilon$ Patzak, $K$ Winter ( $\checkmark$ Spokesperson)

BRUSSELS U, IIHE - P Annis, M Gruwe, C Mommaert,

$M$ Van der Donckt, $P$ Vilain, $G$ Wilquet

FERRARA U \& INFN, FERRARA - E Di Capua, C Luppi, S Ricciardi, B Saitta

TOHO U - T Kawamura, M Kazuno, S Ogawa, II Shibuya

CERN - R Beyer, $J$ Brunner, $M$ de Jong, J P Fabre, R Ferreira,

W Flegel, R Gurin, M Litmaath, L Ludovici, D Macina,

R Meijer Drees, H Meinhard, E Niu, H Overas, J Panman,

F Riccardi, A Rozanov, D Saltzberg, G Stefanini, $R$ Tzcnov,

$C$ Weinheimer, $H$ Wong, $P$ Zucchelli

HAIFA U - J Goldberg, K Hopfner

KINKI U - H Chikawa

BOGAZICI U - E Arik, I Birol, A A Mailov

GYEONGSANG NATIONAL U - C H Hahn, H I Jang, D C Kim,

I G Park, M S Park, J S Song, C S Yoon

AICHI U OF EDUCATION - K Kodama, N Ushida

KOBE U - S Aoki, T Hara

LOUVAIN U - G Brooijmans, D Favart, G Gregoire, J Herin,

$\mathrm{V}$ Lemaitre

MOSCOW, I'TEP - A Artamonov, P Gorbunov, V Khovansky,

V Shamanov, V Smirnitsky

MUNSTER U - D Bonekampcr, D Frekers, D Rondeshagen, $T$ Wolff

NAGOYA U - K Hoshino, M Kobayashi, Y Kotaka, T Kozaki, M Nakamura, $T$ Nakano, K Niu, K Niwa, Y Obayashi, O Sato, 'T Toshito

NAPLES U, IFS \& INFN, NAPLES - S Buontempo, A Cocco, A Ereditato, G Fiorillo, F Garufi, F Marchetti-Stasi,

P Migliozzi, V Palladino, P Strolin

OSAKA CITY U - K Nakamura, T Okusawa, $T$ Yoshida

ROME U \& INFN, ROME - A Capone, $D$ De Pedis, $S$ Di Liberto.

U Dore, P F Loverre, A Maslennikov, M A Mazzoni, F Meddi,

G Piredda, P Righini, R Santacesaria

SALERNO U \& INFN, SALERNO - A di Bartolomeo, G Grella,

G Romano, G Rosa

UTSUNOMIYA U $\sim$ Y Sato, I Tezuka
Accelerator CERN-SPS Detector Spectrometer, Calorimeter

Reactrons

$\nu_{\tau}$ nucleon $\rightarrow \tau^{-} \mathrm{X} \quad 25 \mathrm{GeV}\left(\mathrm{E}_{\mathrm{lab}}\right)$

Brief description The setup consists of a target region, an aircore magnet, a high-precision calorimeter, and a muon spectrometer. Nuclear emulsion stacks form the $800-\mathrm{kg}$ mass of the fiducial target volume. Decays of short-lived particles, such as the $r$, are visualized with higly efficiency. Tracks are located in the cmulsion with high-precision scintillating fiber trackers, and readout with optoelectronic image intensifiers coupled to CCD cameras, thus permitting computer-assisted scanning. The hexagonal aircore magnet provides the measurement of the charge-sign of low energy hadrons and muons. The highprecision calorimeter, which is based on spaghetti technology, tags the $\tau^{-}$decay by its transverse momentum imbalance. The spectrometer identifies muons and measures their momentum and charge. A second phase of data taking with new emulsion stacks is under way (July 96).

Journal papers NIM A344 (1994) 143. NIM A349 (1994) 70. IJMP C5 (1994) 835, NIM A357 (1995) 243, and NIM A367 (1995) 367.

Related experiments CERN-WA-096, FNAL-803

E-mail contact klaus.winter@cern.ch WWW Home-page http://choruswww.cern.ch/welcome.html

\section{CERN-WA-096}

(Proposed 1991, Approved 1991, Began data-taking Apr 1994)

SEARCH FOR THE OSCILLATION $\nu_{\mu} \leftrightarrow \nu_{\tau}$

\section{NOMAD COLLABORATION}

ANNECY - G Bassompierre, J M Gaillard, M Gouanere, $J$ P Mendiburu, L Mossuz, $H$ Pessard, D Sillou, D Verkindt CERN - D Auticro, L Camilleri, L Di Lella ( $\checkmark$ Spokesperson), D Ferrere, A Geiser, J J Gomez-Cadenas, $\Lambda$ Grant, W Huta, L Linssen, P Nedelec, A Placci, B Pope, C Roda, A Rubbia, P Steffen, E Tsesmelis, F Wilson

CALABRIA U - L La Rotonda, M Valdata

DORTMUND U - D Geppert, C Goessling, D Nordmann, D Pollmann, B Schmidt, $T$ Weisse, $K$ Zuber

DUBNA - S Bunyatov, O Klimov, Y Nefedov, B Popov,

S Tereshchenko, $S$ Valuev

FLORENCE U \& INFN, FLORENCE - G Conforto, G Graziani,

E Iacopini, M Kirsanov, A Lupi, A Marchionni, F Martelli, E Pennacchio, C Ricci, M Veltri

HARVARD U .. A Bueno, 'T Dignan, G Feldman, P Hurst. $S$ Mishra

JOHNS HOPKINS U - B Blumenfeld, J Long, D M Steele

LAUSANNE U - K Benslama, I Bird, H Degaudenzi, C Joseph,

M C Nguyen, J P Perroud, G Sozzi, M Steininger, M T Tran, $J M$ Vieira, M Werlen

MELBoURNE U - N Hyett, G Moorhead, C Poulsen, M Sevior,

G Taylor, S Tovey, L Winton

MOSCOW, INR - S Gninenko, A Kovzelev, $\Lambda$ Toropin, S Volkov

PADUA U \& INFN, PADUA - M Baldo-Ccolin, F Bobisut,

G Collazuol, M Contalbrigo, D Gibin, A Guglielmi, S Lacaprara.

M Laveder, M Mezzetto, G Miari, A Sconza, M Vascon,

P Zuccon

PARIS, CURIE UNIV VI \& PARIS, UNIV VII, LPNHE -

$P$ Astier, $M$ Banner, J Dumarchez, E Gangler, A Letessier-

Selvon, J-M Levy, K Schamaneche, A M Touchard, F Vannucci

PAVIA U \& INFN, PAVIA - P Cattaneo, C Conta, R Ferrari,

M Fraternali, A Lanza, M Livan, D Orcstano, F Pastore.

R Petti, G Polesello, A Rimoldi, F Salvatore, V Vercesi

PISA U \& INFN, PISA - C Angelini, V Cavasinni, T Del Prete,

A De Santo, V Flaminio, C Lazzeroni, G Renzoni

DAPNIA, SACLAY - A Baldisseri, J Bouchez, J Gosset,

C Hagner, X Mechain, J P Meyer, A Pluquet, P Rathouit,

T Stolarczyk, M Vo, H Zaccone

SYDNEX, ANSTO - I J Donnelly, K Varvell

SYDNEX U - S Boyd, L Peak, P Soler, J Ulrichs, B Yabsley

UCLA - A Cardini, R Cousins, I Vinogradova, F Weber

MASSACHUSET'S U, AMHERST - $j$ D Hernando

BOS̆KOVIĆ INST, ZAGREB - D Kekez, A Ljubičić, M Stipčević 


\section{SUMMARIES OF CERN EXPERIMENTS}

Accelerator CERN-SPS Detector Spectrometer

Reactions

$$
\begin{array}{lc}
p \text { nucleon } \rightarrow \nu \mathrm{X} & 450 \mathrm{GeV} / \mathrm{c} \\
\nu_{\tau} \text { nucleon } \rightarrow \tau \mathrm{X} & -
\end{array}
$$

Particles studied $\nu$

Brief description Searches for the oscillation $\nu_{\mu} \leftrightarrow \nu_{T}$ in a wide band, $10-200 \mathrm{GeV}$ neutrino beam. Aims at detecting $\nu_{\tau}$ charged-current interactions by observing the production of the $\tau$ through its various decay modes by means of kinematical criteria. The detector reconstructs the event kinematics. It uses the CERN-UA-001 magnet. The target consists of 44 drift chamber planes with a total mass of 2.9 tons over a fiducial volume of $2.6 \times 2.6 \times 4 \mathrm{~m}^{3}$. It is followed by transition radiation detectors, by additional tracking chambers and by an electromagnetic calorimeter consisting of 875 lead glass blocks and including a preshower detector. A hadronic calorimeter made of iron and scintillator is located behind the electromagnetic calorimeter outside of the magnetic field volume. A muon detector has two stations of large-area drifttube chambers on both sides of an iron absorber. The sensitivity of the detector to $(\Delta m)^{2}$ is larger than $1 \mathrm{eV}^{2}$. Taking data (July 96). Expected to continue data taking until the end of 1997.

Journal papers NIM A372 (1996) 556, and NIM A373 (1996) 358

Related experiments CERN-WA-095, FNAL-803

E-mail contact luigi.di.lella@cern.ch

WWW Home-page inttp://nomadinfo.cern.ch/

\section{CERN-WA-102}

(Proposed Sep 1994, Approved Sep 1994, Began data-taking Apr 1995, In progress)

A SEARCH FOR CENTRALLY PRODUCED NON$q \bar{q}$ MESONS IN PROTON PROTON INTERACTIONS AT $450 \mathrm{GeV} / c$ USING THE $\Omega$ SPECTROMETER AND GAMS -4000

WA102 COLLABORATION

ANNECY - 3 P Peigneux, M Poulet

ATHENS U - M Spyropoulou-Stassinaki, G Vassiliadis

BERGEN U - K Myklebost, J M Olsex

BIRMINGHAM U - J N Carney, J P Davies, D Evans,

J B Kinson, K Norman, M Venables, O Villalobos Baillie,

M F Votruba

BRUSSELS U, IISN - F G Binon, J M Frere, J P Stroot

CERN - W Beusch, B R French, A Jacholkowski, A Kirk

( $\checkmark$ Spokesperson), W Klempt, P Martinengo, E Quercigh, H Rotscheidt, M Sene, R Sene

DUBNA - Y Kulchitsky, S Maljukov, I Minashvili, V Romanovsky,

V Roumiantsev, N Russakovich, A Semenov, A Soloviev, G Tchlatchidze

KEK - S Inaba, T Ishida, T Kinashi, $T$ Nakagawa, H Shimizu,

K Takamatsu, T 'Tsuru, Y Yasu

UNIVERSITY COLL, LONDON - G Crone, M Esten

LOS ALAMOS - D Alde, E A Knapp, T Lopez

MANCHESTER U - I Duerdoth, N Lumb, S Snow, R Thompson

OSLO U - K Danielsen, 'T Jacobsen

SERPUKHOV - A V Dolgopolov, S V Donskov, A V Inyakin,

G V Khaustov, V Kolossov, A A Kondashov, A A Lednev,

$\checkmark$ A Polovnikov, S A Polyakov, Y D Prokoshkin, S A Sadovsky,

V D Samoylenko, P M Shagin, A V Shtannikov, A V Singovsky,

V P Sugonyacv

Accelerator CERN-SPS Detector OMEGA, GAMS-4000

\section{Reactions}

$$
p p \rightarrow p p \mathrm{X} \quad 450 \mathrm{GeV} / c\left(\mathrm{P}_{\mathrm{lab}}\right)
$$

Particles studied mesons

Brief description Proposes to perform two 100 day runs

combining the multiphoton detection of GAMS- 4000 with the charged particle detcction of the Omega Spectrometer to search for non- $q \bar{q}$ mesons in the reaction $p p \rightarrow p_{f} X^{0} p_{s}$ at $450 \mathrm{GeV} / c$. Here, $p_{f}$ and $p_{s}$ stand for the fastest and the slowest particles in the lab system. Particular attention will be paid to the final states decaying to $\eta \eta, \eta \eta^{\prime}$ and $\eta^{\prime} \eta^{\prime}$. The physics goal is a better understanding of non-perturbative QCD. Taking data (July 96). Related experiments CERN-WA-076, CERN-WA-091

E-mail contact andrew.kirk@cern.ch

\section{CERN-LHC-ATLAS}

(Proposed Oct 1992, Dec 1994, Approved Jan 1996, In preparation)

\section{A TOROIDAL LHC APPARATUS - ATLAS}

\section{ATLAS COLLABORATION}

Accelerator CERN-LHC Detector ATLAS

Brief description ATLAS is a general purpose experiment for recording the proton-proton collisions at LHC. The detector design has been optimized to cover searches for Higgs bosons, alternative schemes of the symmetry breaking, searches for supersymmetric particles and other new particles, study of compositeness of quarks and leptons, $C P$ violation in the $B$ sector, measurements of the properties of the third family of quarks, and more. The apparatus includes an inner tracking detector inside a 2-tesla solenoid, electromagnetic and hadronic calorimeters outside the solenoid and in the forward regions, and barrel and endcap air-core-toroid muon spectrometers. Under construction (July 96). The collaboration consists of more than 1500 scientists, engineers, and graduate students from 148 participating institutions. For further details, please contact the Spokesperson, Dr. Peter Jenni [CERN], or the Deputy Spokesperson, Dr. Torsten Akesson [Lund U.]

E-mail contact peter.jenni@cern.ch, torsten.akesson@quark.lu.se WWW Home-page http://atlasinfo.cern.ch/Atlas/Welcome.html

\section{CERN-LHC-CMS}

(Proposed Oct 1992, Approved Jan 1996, in preparation)

\section{THE COMPACT MUON SOLENOID - CMS}

\section{CMS COLLABORATION}

\section{Accelerator CERN-LHC Detector CMS}

Brief description CMS is a general purpose detector designed to run at the highest luminosity at the LH.C. It has been optinized for the search of the Standard Model Higgs boson over a mass range from $90 \mathrm{GeV}$ to $1 \mathrm{TeV}$. but it also allows detection of a wide range of possible signatures from alternative electroweak symmetry breaking mechanisms. Furthermore, it is well adapted for the study of top, beauty and tau physics at lower luminosities and will cover several important aspects of the heavy ion physics program. CMS identifies and measures muons, photons and electrons with high precision. At the core of the detector sits a large superconducting solenoid generating a uniform magnetic field of $4 \mathrm{~T}$. The strong magnetic field allows a compact design for the muon spectrometer without compromising the momentum resolution. The central tracker consists of silicon pixels, silicon microstrips and microstrip gas chambers. The electromagnetic calorimeter is based on Lead Tungstate crystals. Under construction (July 96). The collaboration consists of almost 1500 scientists, engineers, and graduate students from 134 participating institutions in 30 countries. For further details, please contact the Spokesperson, Dr. Michel della Negra [CERN], or the Deputy Spokesperson, Dr. Tejinder S. Virdee [Impcrial Coll.]

E-mail contact michel.della.negra@cern.ch, tejinder.virdee@cern.ch

WWW Home-page

http://cmsinfo.cern.ch/cmsinfo/Welcome.html 


\section{SUMMARIES OF CERN EXPERIMENTS}

\section{Other CERN Experiments}

Listed here are some other CERN experiments of interest to the particle physics community. Find more details about these projects online, in the SLAC's EXPERIMENTS database (see p. 3), or contact the spokespersons. Check also the annual report 'Experiments at CERN', which lists all the current CERN experiments and $\mathrm{R} \& \mathrm{D}$ projects. Find additional information at the Web site: http://www.cern.ch/CERN/Experiments.html

CERN-EMU-011 (Taking data)

STUDY OF PARTICLE PRODUCTION AND NUCLEAR FRAGMENTATION IN RELATIVISTIC HEAVY ION COLLISIONS IN NUCLEAR EMULSION

Spokesperson: P.L. Jain [SUNY, Buffalo]

E-mail contact phyjain@ubvms.cc.buffalo.edu

CERN-EMU-012 (Taking data)

PARTICLE PRODUCTION, DENSITY FLUCTUA

TIONS, AND BREAK UP OF DENSE NUCLEAR MATTER IN CENTRAL $\mathrm{Pb}$ Ag AND Pb Pb INTERACTIONS AT $60-160$ A GeV

Spokesperson: I. Otterlund [Lund U.]

E-mail contact ingvar.otterlund@kosufy.lu.se

CERN-EMU-013 (Completed data-taking)

INTERACTIONS OF $160 \mathrm{GEV} /$ NUCLEON ${ }^{20} \mathrm{~Pb}$ NUCLEI IN EMULSION CHAMBERS WITH COPPER AND LEAD TARGETS

By KLMM Collaboration

Spokesperson: W. Wolter [Cracow, INP-Exp]

E-mail contact wwolter@vsk01.ifj.cdu.pl

CERN-EMU-014 (Taking data)

STUDY OF MULTIPLICITY AND ANGULAR CHARACTERISTICS IN Pb + A INTERACTION AT $200 \mathrm{~A}$ $\mathrm{GeV} / c$

Spokesperson: D. Ghosh [Jadavpur U.]

CERN-EMU-015 (Taking data)

INVESTIGATION OF CENTRAL Pb Pb INTERAC

TIONS AT ENERGIES OF $160 \mathrm{GeV} /$ NUCLEON WITH

THE HELP OF THE EMULSION MAGNETIC CHAM-

BER

Spokesperson: K.A. Kotelnikov [Lebedev Inst.]

E-mail contact koteln@sci.fian.msk.su

CERN-EMU-016 (Taking data)

ISOSPIN CORRELATIONS IN HIGH ENERGY Pb Pb INTERACTIONS

Spokesperson: Y. Takahashi [Alabama U., Huntsville]

E-mail contact takahashi@ssl.msfc.nasa.gov

CERN-EMU-017 (Completed data-taking Dec 1994)

FRAGMENTATION OF Pb PROJECTILES AT SPS

ENERGIES

Spokesperson: Wolfgang Heinrich [Siegen U.]

E-mail contact heinrich@hrz.uni-siegen.d400.de
CERN-EMU-018 (Taking data)

EXPOSURES OF CR39 STACKS TO LEAD IONS AT THE CERN-SPS

Spokesperson: G. Giacomelli [Bologna U. and INFN, Bologna]

E-mail contact giacomelli@bologna.infn.it

CERN-EMU-019 (Taking data)

NUCLEAR FRAGMENTATION INDUCED BY RELATIVISTIC PROJECTILES STUDIED IN THE $4 \pi$ CONFIGURATION OF PLASTIC $\Upsilon R A C K$ DETECTORS

Spokesperson: H.A. Khan [Pinstech, Islamabad]

CERN-EMU-020 (laking data)

$\bar{p}$-INDUCED FISSION STUDIES WITH PLASTIC TRACK DETECTORS USING $4 \pi$ GEOMETRY

Spokesperson: H.A. Khan [Pinstech, Islamabad]

CERN-NA-035 (Completed data-taking May 1992) STUDY OF RELATIVISTIC NUCLEUS-NUCLEUS COLLISIONS

By NA35 Collaboration

Spokesperson: Peter Seyboth [Munich, Max Planck Inst.]

E-mail contact pxs@dmumpiwh.mppmu.mpg.de

WWW Home-page http://hpna49-1.cern.ch/na35.html

CERN-NA-038 (Completed data-taking May 1992)

STUDY OF HIGH-ENERGY NUCLEUS-NUCLEUS

INTERACTIONS WITH THE ENLARGED NA10

DIMUON SPECTROMETER

Spokesperson: Louis Kluberg [Ecole Polytechnique, LPNHE]

E-mail contact louis.kluberg@cern.ch

WWW Home-page http://www.cern.ch/NA38/

CERN-NA-043-2 (Taking data)

INVESTIGATIONS OF THE COHERENT HARD

PHOTON YIELDS FROM 50-300 GeV $/ c e^{ \pm}$IN STRONG CRYSTALLINE FIELDS OF DIAMOND, Si, AND Ge CRYSTALS

Spokesperson: Erik Uggerhoj [Aarhus U.]

E-mail contact isa@dfiaau.dk

CERN-NA-044 (Taking data)

A FOCUSSING SPECTROMETER FOR ONE AND TWO PARTICLES

Spokesperson: Hans Boggild [Bohr Inst.]

E-mail contact boggild@nbivax.nbi.dk, hans.boggild@cern.ch WWW Home-page http://p2hp2.lanl.gov/na44/na44.html

CERN-NA-045 (Completed data-taking Apr 1995)

STUDY OF LOW-MASS ELECTRON PAIR PRODUCTION IN HADRON AND NUCLEAR COLLISIONS AT THE CERN SPS

By CERES Collaboration

Spokesperson: Itzhak Tserruya [Weizmann Inst.]

E-mail contact fntsruya@weizmann.weizmann.ac.il, itzhak.tserruya@ern.ch

WWW Home-page http://ceres6.physi.uni-heidelberg.de/ 


\section{SUMMARIES OF CERN EXPERIMENTS}

CERN-NA-049 (Taking data)

LARGE ACCEPTANCE HADRON DETECTOR FOR AN INVESTIGATION OF Pb-INDUCED REACTIONS AT THE CERN SPS

Spokesperson: Reinhard Stock [Frankfurt U.]

E-mail contact stock@ikf.physik.uni-frankfurt.de,

reinhard.stock@cern.ch

WWW Home-page http://hpna49-1.cern.ch/na49.html

\section{CERN-NA-052 (Taking data)}

STRANGELET AND PARTICLE SEARCH IN Pb Pb COLLISIONS

Spokesperson: Klatus Pretzl [Bern U.]

E-mail contact klaus.pretzl@cern.ch

WWW Home-page http://www.lhep.unibe.ch/newmass/

\section{CERN-NA-053 (Taking data)}

ELECTROMAGNETIC DISSOCIATION OF TARGET

NUCLEI BY ${ }^{208} \mathrm{~Pb}$ PROJECTILES

Spokesperson: J.C. Hill [Iowa State U.]

E-mail contact jhill@iastate.edu

CERN-NA-054 (Taking data)

DETERMINATION OF CROSS-SECTIONS OF FAST-

MUON INDUCED REACTIONS TO COSMOGENIC

RADIONUCLIDES

Spokesperson: E. Nolte [Munich, Tech U.]

CERN-NA-055 (In preparation)

INVESTIGATION OF FAST NEUTRON PRODUCTION BY 100 TO $250 \mathrm{GeV}$ MUON INTERACTION ON THIN TARGETS

Spokesperson: N. Mascarenhas [Cal Tech]

CERN-NA-056 (In preparation)

MEASUREMENT OF PION AND KAON FLUXES BELOW $60 \mathrm{GeV} / \mathrm{c}$ PRODUCED BY $450 \mathrm{GeV} / \mathrm{c}$ PROTONS ON A BERYLLIUM TARGET

By SPY Collaboration

Spokesperson: S. Ragazzi [Milan U. and INFN, Milan] WWW Home-page http://www.cern.ch/NA56/

CERN-PS-185-3 (In preparation)

A MEASUREMENT OF DEPOLARIZATION AND SPIN TRANSFER IN $\bar{p} p \rightarrow \bar{\Lambda} \Lambda$

Spokesperson:

Klaus Rochrich [Julich, Forschungszentrum] WWW Home-page

http://hpfr02.physik.uni-freiburg.de/psi85/ps185.html

CERN-PS-189 (Completed data-taking Dec 1992)

HIGH PRECISION MASS MEASUREMENTS WITH

A RADIOFREQUENCY MASS SPECTROMETER -

APPLICATION TO THE MEASUREMENT OF THE $p \bar{p}$

MASS DIFFERENCE

Spokesperson: Catherine 'Thibault [Orsay, CSNSM]

E-mail contact catherine.thibault@cern.ch
CERN-PS-194-3 (Taking data)

MEASUREMENT OF STOPPING POWERS AND SINGLE IONXZATION CROSS-SECTIONS FOR ANTIPROTONS AT LOW ENERGIES

Spokesperson: Erik Uggerhoj [Aarhus U.]

CERN-PS-200 (Taking data)

CAPTURE, ELECTRON COOEING, AND COMPRESSION OF ANTIPROTONS IN A LARGE PENNING TRAP AND PHYSICS EXPERIMENTS WITH AN ULTRA-LOW-ENERGY EXTRACTED ANTIPROTON BEAM

Spokespersons: M.H1. Holzscheiter, M.M. Nieto [Los Alamos] E-mail contact mhh@lanl.gov

CERN-PS-203 (Completed data-taking Dec 1993)

ANTIPROTON INDUCED FISSION AND FRAGMENTATION OF NUCLEI

Spokesperson: T, von Egidy [Munich, Tech. U.]

E-mail contact till.v.egidy@physik.tu-muenchen.de

CERN-PS-205 (Taking data)

LASER SPECTROSCOPY OF ANTIPROTONIC HE-

LIUM ATOMS

By PS205 Collaboration

Spokesperson: T. Yamazaki [Tokyo U., INS]

$\underline{E-m a i l ~ c o n t a c t}$ yamazaki@insie1.ins.u-tokyo.ac.jp, eades@vxcern.cern.ch

WWW Ilome-page http://www.cern.ch/LEAR_PS205/

CERN-PS-206 (Completed data-taking Sep 1993)

MEASUREMENT OF THE $\bar{p} p \rightarrow \bar{n} n$ CHARGEEXCHANGE DIFFERENTIAL CROSS SECTION By CCX Collaboration

Spokesperson: Franco Bradamante [Trieste U. and INFN, Irieste] $\underline{\text { E-mail contact }}$ franco.bradamante@cern.ch

CERN-PS-207 (Taking data)

PRECISION MEASUREMEN'T OF THE ENERGIES AND LINE SHAPES OF ANTIPROTONIC LYMAN AND BALMER TRANSITIONS FROM HYDROGEN AND HELIUM ISOTOPES

Spokesperson: D. Gotta [Julich, Forschungszentrum]

CERN-PS-209 (Taking data)

NEUTRON HALO AND ANTIPROTON-NUCLEUS POTENTIAL FROM ANTIPROTONIC $X \cdot$ RAYS

Spokesperson: J. Jastrzebski [Warsaw U., Heavy Ion Lab]

CERN-PS-210 (Taking data)

ANTIHYDROGEN PRODUCTION IN $\bar{p} Z$ INTERACTION

Spokesperson: W. Oelert [Julich, Forschungszentrum]

E-manl contact w.oelert@kfa-juelich.de

WWW Home-page http://ikpellol.ikp.kfa-juelich.de/ps210/ 
SUMMARIES OF CERN EXPERIMENTS

CERN-PS-211 (In preparation)

EXPERIMENTAL STUDY OF THE PHENOMENOLOGY OF SPALLATION NEUTRONS IN A LARGE LEAD BLOCK

Spokesperson: C. Rubbia [CERN]

E-mail contact carlo.rubbia@cern.ch

CERN-PS-212 (In preparation)

LIFETIME MEASUREMENT OF $\pi^{+} \pi^{-}$ATOMS TO TEST LOW-ENERGY QCD PREDICTIONS

Spokesperson: L. Nemenov [Dubna, JINR]

E-mail contact nemenov@nu.jinr.dubna.su

CERN-WA-084 (Completed data-taking Sep 1991)

STUDY OF THE PRODUCTION AND DECAY PROP. ERTIES OF BEAUTY FLAVORED HADRONS

Spokesperson: Giuseppe Martellotti [Rome U. and INFN, Rome]

E-mail contact martellotti@roma1.infn.it

CERN-WA-097 (Taking data)

STUDY OF BARYON AND ANTIBARYON SPECTRA IN $\mathrm{Pb} P$ P INTERACTIONS AT $160 \mathrm{GeV} / c$ PER NU. CLEON

Spokesperson: Emanuele Quercigh [CERN]

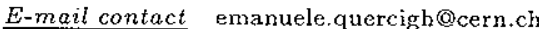

CERN-WA-098 (Taking data)

LARGE ACCEPTANCE MEASUREMENT OF PHO TONS AND CHARGED PARTICLES IN HEAVY ION REACTIONS

Spokesperson: Hans H. Gutbrod [Darmstadt, GSI]

E-mail contact gutbrod@vxwa80.cern.ch

WWW Home-page http://www.cern.ch/WA98/Welcome.html

CERN-WA-099 (Completed data-taking Apr 1995)

MEASUREMENTS OF PAIR PRODUCTION AND ELECTRON CAPTURE FROM THE CONTINUUM IN HEAVY PARTICLE COLLISIONS

Spokesperson: S. Datz [Oak Ridge]

E-mail contact datzs@ornl.gov

CERN-WA-099-2 (Taking data)

CHARGE CHANGING COLLISIONS, ENERGY LOSS AND EM NUCLEAR REACTIONS OF $160 \mathrm{GeV} \mathrm{A}{ }^{208} \mathrm{~Pb}$

Spokesperson: S. Datz [Oak Ridge]

E-mail contact datzs@ornl.gov

CERN-WA-101 (Taking data)

STUDY OF VARIOUS PROCESSES WITH A $160 \mathrm{GeV} / c$ PER NUCLEON Pb BEAM

Spokesperson: Yudong He [UC, Berkeley]

E-mail contact yudong@physics.berkeley.edu

\section{CERN Future Plans}

CERN's main project for the start of the next millennium is the LHC proton-proton collider in the 27-kilometer LEP tunncl, currently scheduled to begin experiments in 2005. Besides the LHC, the ongoing program also continues CERN's tradition of serving a wide research community. The LEP electron-positron collider, after six years of running at and around the $Z$ resonance, has been equipped with superconducting accelerating cavities, thereby becoming LEP2, and began operating in Summer 1996 at a collision energy of $161 \mathrm{GeV}$, the threshold to produce pairs of $W$ bosons. The collision energy of LEP 2 is being further increased as additional superconducting cavities are added. The collider is scheduled to operate till the end of 1999. At the SPS proton synchrotron, flagship experiments continue with neutrino and muon beams and in the study of $C P$ violation. In addition, the heavy ion program at the SPS serves a large range of experiments for a separate research community. While the LEAR low energy antiproton ring is being closed in 1996, a new facility is being foreseen to supply a modest level of antiprotons for experiments on 'trapped' antiparticles. The ISOLDE on-line isotope separator, served by the $1 \mathrm{GeV}$ Booster synchrotron, caters to yet another group of rescarchers. 


\section{SUMMARIES OF CORNELL EXPERIMENTS}

\section{CESR Experiments}

\section{CESR-CLEO}

(Proposed 1975, Approved 1977, Began data-taking Oct 1979, In progress)

\section{THE CLEO EXPERIMENT AT CESR}

\section{CLEO COLLABORATION}

CAL TECH - B Barish, M Chadha, S Chan, G Eigen, J S Miller, C O'Grady, M Schmidtler, J Urheim, A J Weinstein, F Wuerthwein

CARLETON U - K W Edwards

COLORADO U - R Balest, B H Behrens, K Cho, W T Ford, H Park, P Rankin, J Roy, J G Smith

CORNELL U - J P Alexander, C Bebek, B E Berger,

K Berkelman, K Bloom, D G Cassel, H A Cho, D M Coffman, D S Crowcroft, M Dickson, P S Drell, K M Ecklund, R Ehrlich, R Elia, $A$ Foland. P Gaidarev, R S Galik, B Gittelman, SW Gray, D L Hartill, B K Heltslcy, P Hopman, S L Jones, $\mathrm{J}$ Kandaswamy, P C Kim, D L Kreinick, 'T Lee, Y Liu, G S Ludwig, J Masui, J Mevissen, N B Mistry, C R Ng, E Nordberg, M Ogg, J R Patterson, D Peterson, D Riley, A Soffer, B Valant-Spaight, C Ward

FLORIDA U - M Athanas, P Avery, C D Jones, M Lohner, C Prescott, J Yelton

IIARVARD U - G Brandenburg, R A Briere, Y Gao, D Kim, $R$ Wilson, II Yamamoto

HAWAII U - T E Browder, F Li, Y Li, J Rodriguez

ILLINOIS U, URBANA - T Bergfcld, B I Eisenstein, J Ernst,

G E Gladding, G D Gollin, R M Hans, E Johnson, M Marsh, M Palmer, M Selen, J J Thaler

MCGILL U - A Bellerive, D I Britton, R Janicek,

D B MacFarlane, $\mathrm{K} \mathrm{W}$ McLean, $P$ M Patel

ITHACA COLL - A J Sadoff

KANSAS U .. R Ammar, P Baringer, A Bean, D Besson,

D Coppage. C Darling, R Davis, N Hancock, S Kotov,

I Kravchenko, N Kwak

MINNESOTA U - S Anderson, Y Kubota, M Lattery, S Lee,

J J O'Neill, S Patton, R A Poling ( $\checkmark$ Spokesperson), T Riehle.

V Savinov, A Smith

SUNY, ALBANY - M S Alam, B Athar, $Z$ Ling, A H Mahmood, H Severini, $S$ Timm, F Wappler

OHIO STATE U - A Anastassov, J E Duboscq, D Fujino, K K Gan, T Hart, K Honscheid, H Kagan, R Kass, J Lee, M Spencer, M Sung, A Undrus, A Wolf, M M Zoeller

OKLAHOMA U - B Nemati, S J Richichi, W R Ross, P Skubic, $M$ Wood

PURDUE U - M Bishai, J Fast, E Gerndt, J W Hinson, N Menon, D H Miller, E I Shibata, I P I Shipsey. M Yurko

ROCHESTER U - L Gibbons, S D Johnson. Y Kwon, S Roberts, E H Thorndike, L Tian

SLAC - C P Jessop, K Lingel, H Marsiske, M L Per!, S F Schaffner, R Schindler, D Ugolini, R Wang, X Xhou

SOUTHERN METHODIS'T U -- T E Coan, V Fadeyev I Korolkov, Y Maravin, I Narsky, V Shelkov, J Stacck, R Stroynowski, I Volobuev, J Yc

SYRACUSE U - M Artuso, A Efimov, F Frasconi, M Gao, $M$ Goldberg, $R$ Greene, D He, N Horwitz, S Kopp, G C Moneti, R Mountain, Y Mukhin, S Schuh, T Skwarnicki, S Stone, $G$ Viehauser, $X$ Xing

UC, SAN DIEGO D M Asner, D W Bliss, W S Brower, G Masek, H P Paar, V Sharma, M Sivertz

UC, SANTA BARBARA J Gronberg, $R$ Kutschke, $D I$ Lange, $S$ Menary, R J Morrison, H N Nelson, T K Nelson, C Qiao, J D Richman, D Roberts, $A$ Ryd, M S Witherell, A Wu VANDERBILT U - J Bartelt, S E Csorna, V Jain, S Marka VIRGINIA TECII - R Godang, K Kinoshita, I C Lai,

P Pomianowski, S Schrenk

WAYNE STATE U - G Bonvicini, D Cinabro. L Perera, G Sun, G J Zhou

Accelerator CESR Detector CLEO

\author{
Reactions \\ $e^{+} e^{-} \rightarrow$ hadrons \\ $e^{+} e^{-} \rightarrow e^{+} e^{-}$ \\ $e^{+} e^{-} \rightarrow \mu^{+} \mu^{-}$ \\ $e^{+} e^{-} \rightarrow e^{+} e^{-}$hadrons \\ " \\ $e^{+} e^{-} \rightarrow \tau^{+} \tau^{-}$ \\ Particles studied $\Upsilon(1 S), \Upsilon(2 S), \Upsilon(3 S), \Upsilon(4 S), B, \tau, D^{+}, D^{0}$, \\ $D_{s}^{+}$, charmed-baryon
}

Brief description Since 1979 the collaboration has conducted

studies of $b, c, \tau$ and $\gamma \gamma$ physics in $e^{+} e^{-}$interactions near $10 \mathrm{GeV}$. Current topics include determination of the CKM parameters and the Standard Model tests in decays of heavy fiavors, as well as QCD tests in a varicty of processes. Successive detector upgrades have kept pace with luminosity improvements to the Cornell Electron Storage Ring (CESR), which has delivered over $6 \mathrm{fb}^{-1}$ of integrated luminosity to date. The CLEO. II detector (proposed 1983, approved 1984, operational since 1989) consists of drift chambers for tracking charged particles and measuring $\mathrm{d} E / \mathrm{d} x$, time-of-flight counters, a 7800 -element CsI electromagnetic calorimeter, a 1.5 tesla superconducting solenoid, iron for flux return and muon identification, and muon chambers. A three-layer silicon vertex detector was added in the Fall of 1995. Taking data (July 96). A major upgrade, the CLEO-III detector, was proposed and approved in 1994, and the installation is planned for 1998.

Journal papers PRL 44 (1980) 1108, PRL 45 (1980) 219, PRL 46 (1981) 84, PRL 46 (1981) 88, PRL 46 (1981) 1181, PRL 48 (1982) 1070, PRL 49 (1982) 357, PRL 49 (1982) 610, PRL 49 (1982) 617, NIM 211 (1983) 47, PL B222 (1983) 317, PRL 50 (1983) 807, PRL 50 (1983) 877, PRL 50 (1983) 881, PRL 51 (1983) 347, PRL 51 (1983) 634, PRL 51 (1983) 1139, PRL 51 (1983) 1143, PR D27 (1983) 475, PR D27 (1983) 1665, PL B137 (1984) 277, PRL 52 (1984) 799, PRL 52 (1984) 1084, PRL 53 (1984) 24, PRL 53 (1984) 1309, PR D29 (1984) 1285, PR D30 (1984) 1433. PR D30 (1984) 1996, PR D30 (1984) 2279, PRL 54 (1985) 381. PRL 54 (1985) 1894 , PRL 55 (1985) 923, PRL 55 (1985) 1248, PR D31 (1985) 2161, PR D31 (1985) 2386, PR D32 (1985) 2294, PR D32 (1985) 2468, PRL 56 (1986) 800, PRL 56 (1986) 1222, PRL 56 (1986) 1893, PRL 56 (1986) 2676, PRL 56 (1986) 2781, PR D33 (1986) 300, PR D34 (1986) 905, PR D34 (1986) 3279, PL B183 (1987) 429, PL B191 (1987) 318, PRL 58 (1987) 183, PRL 58 (1987) 307, PRL 58 (1987) 1814, PRL 59 (1987) 22, PRL 59 (1987) 407, PRL 59 (1987) 1993, PR D35 (1987) 19, PR D35 (1987) 1081, PR D35 (1987) 2747, PR D35 (1987) 3533, PR D36 (1987) 690, PR D36 (1987) 1289, PRL 60 (1988) 1614, PR D37 (1988) 1719 ferratum: PR D39 (1989) 1471], PR D38 (1988) 2679 [erratum: PR D40 (1989) 1701], PL B223 (1989) 470, PL B224 (1989) 445, PL B226 (1989) 192, PL B226 (1989) 401, PRL 62 (1989) 8, PRL 62 (1989) 863, PRI 62 (1989) 1240, PRL 62 (1989) 2233, PRL 62 (1989) 2436, PRL 63 (1989) 1667, PR D39 (1989) 3528, PR D40 (1989) 263, PR D40 (1989) 712 ferratum: PR D40 (1989) 3790], PL B243 (1990) 169, PL B251 (1990) 223, PRL 64 (1990) 16, PRL 64 (1990) 2117, PRL 64 (1990) 2226, PRL 65 (1990) 1184, PRL 65 (1990) 1531, PRL, 65 (1990) 2842, PR D4I (1990) 805, PR D41 (1990) 774, PR D4l (1990) 1401, NIM A302 (1991) 261, PRL 67 (1991) 1692, PRL 67 (1991) 1696, PR D43 (1991) 651, PR D43 (1991) 1448, PR D43 (1991) 2836, PR D43 (1991) 3599, PR D44 (1991) 593, PR D44 (1991) 3383, PR D44 (1991) 3394 , NIM A320 (1992) 66, PL B283 (1992) 161, PL B291 (1992) 488 PL B294 (1992) 139, PRL 68 (1992) 1275, PRL 68 (1992) 1279, PRL 69 (1992) 2041, PRL 69 (1992) 2046, PRL 69 (1992) 3278, PRL 69 (1992) 3610 (erratum: PRL 71 (1993) 3395], PR D45 (1992) 1, PR D45 (1992) 21, PR D45 (1992) 752, PR D45 (1992) 2212, PR D45 (1992) 3965, PR D45 (1992) 3976, PR D46 (1992) 4822, MPL A8 (1993) 869, PL B303 (1993) 377, PL B317 (1993) 647, PL B319 (1993) 365, PRL 70 (1993) 138, PRL 70 (1993) 1207, PRL 70 (1993) 2681, PRL 70 (1993) 3700, PRL 71 (1993) 674, PRL 71 (1993) 1311, PRL 71 (1993) 1680, PRL 71 (1993) 1791, PRL 71 (1993) 1973, PRL 71 (1993) 2391, PRL 71 (1993) 3070 , PRL 71 (1993) 3255, PRL 71 (1993) 3259, PRL 71 (1993) 3922, PRL 71 (1993) 4111, PR D47 (1993) 791, PR D47 (1993) 3671 , PR D48 (1993) 4007. NIM A345 (1994) 429, NIM A351 (1994) 19, NIM A351 (1994) 43, PL B323 (1994) 219, PL B324 (1994) 255, PL B325 (1994) 257, PL B331 (1994) 236 lerratum: PL B342 (1995) 453], PL B337 (1994) 405, PL B340 (1994) 129, PE B340 (1994) 194, PRL 72 (1994) 1406, PRL 72 (1994) 


\section{SUMMARIES OF CORNELL EXPERIMENTS}

1972, PRL 72 (1994) 2328, PRL 72 (1994) 3762, PRL 73 (1994)

934, PRL 73 (1994) 1079, PRL 73 (1994) 1472, PRL 73 (1994)

1890, PRL 73 (1994) 3503 [erratum: PRL 74 (1995) 3090], PR D49 (1994) 40, PR D49 (1994) 5690, PR D49 (1994) 5701, PR

D50 (1994) 43, PR D50 (1994) 1884, PR D50 (1994) 3027, PR

D50 (1994) 4265, PR D50 (1994) 5484, NIM A368 (1995) 68,

PL B341 (1995) 435 [erratum: PL B347 (1995) 469], PL B350

(1995) 256, PL B356 (1995) 580, PRL 74 (1995) 2885, PRL 74

(1995) 3113 [erratum: PRL 75 (1995) 4155], PRL 74 (1995)

3331, PRL 74 (1995) 3534, PRL 75 (1995) 624, PRL 75 (1995)

785, PRL 75 (1995) 3232, PRL 75 (1995) 3804, PRL 75 (1995)

3809, PRL 75 (1995) 4364, PR D51 (1995) 1014, PR D51 (1995)

2053, PR D52 (1995) 2656, PR D52 (1995) 2661, PR D52 (1995)

4860, PL B365 (1996) 431, PL B369 (1996) 186, PL B373 (1996)

261, PL B373 (1996) 334, PRL 76 (1996) 1570, PRL 76 (1996)

2637, PRL 76 (1996) 3065, PRL 76 (1996) 3898, PRL 76 (1996)

4119, PR D53 (1996) 1013, PR D53 (1996) 1039, PR D53 (1996)

4734, PR D53 (1996) 6033, and PR D53 (1996) 6037.

E-mail contact rap@Ins62.Ins.cornell.edu

WWW Home-page http://w4.lns.cornell.edu/

\section{CESR Future Plans}

The Cornell Electron Storage Ring (CESR) is currently delivering peak luminosities of $4 \times 10^{32} \mathrm{~cm}^{-2} \mathrm{~s}^{-1}$. In 1998, operations will shut down for detector and machine upgrades that will enable the machine to deliver peak luminosities in excess of $1.5 \times 10^{33}$ $\mathrm{cm}^{-2} \mathrm{~s}^{-1}$. The detector modifications will include new tracking devices and a high performance fast RICH detector with $\pi / K$ separation out to the kinematic limit for $B$ meson daughters. When CESR starts running again in late 1998, the physics focus of the upgrade will be rare $B$ decays and $C P$ violation. $\mathrm{R} \& \mathrm{D}$ is currently underway to further increase the luminosity of the storage ring to $10^{34} \mathrm{~cm}^{-2} \mathrm{~s}^{-1}$, and the CESR/CLEO plans promise a rich physics program well into the next century. 


\section{SUMMARIES OF DESY EXPERIMENTS}

\section{DESY Experiments}

\section{DESY-DORIS-ARGUS}

(Proposed 1978, Approved 1979, Began data-taking Sep 1982,

Completed data-taking Oct 1992)

\section{ARGUS - A NEW DETECTOR FOR DORIS}

\section{ARGUS COLLABORATION}

DESY - H Albrecht, $\Upsilon$ Hamacher, R P Hofmann, 'T Kirchhoff, R Mankel, A Nau, S Nowak, D Ressing, H Schroeder ( $\sqrt{ }$ Spokesperson), H D Schulz, M Walter, R Wurth

DORTMUND U -. C Hast, H Kapitza, H Kolanoski, A Kosche, A Lange, A Lindner, M Schieber, T Siegmund, H Thurn, $\mathrm{D}$ Toepfer, D Wegener

DRESDEN, TECH U - C Frankl, M Schmidtler, M Schramm,

$K R$ Schubert, R Schwierz, B Spaan, R Wald

ERLANGEN U - K Reim, H Wegener

HAMBURG U -. R Eckmann, H Kuipers, O Mai, R Mundt,

$T$ Oest, $R$ Reiner, W Schmidt-Parzefall

HEIDELBERG U, IHEP - J Stiewe, S Werner

HEIDELBERG, MAX PLANCK INST - K Ehret, W Hofmann, A Huepper, K T Knoepfle, J Spengler

IPP, CANADA \& MCGILL U \& TORONTO U - P Krieger, D B MacFarianc, J D Prentice, P R B Saull, K Tyamariudaki, $R$ Van de Water, ' $\mathrm{I}$ S Yoon

KARLSRUHE $U-M$ Schneider, S Weseler

SIEFAN INST, LJUBLJANA \& LJUBLJANA U - M Bracko,

G Kernel, P Krizan, E Kriznic, G Medin, 'I' Podobnik, 'T Zivko

MOSCOW, I'TEP - V Balagura, S Barsuk, I Belyaev, $R$ Chistov,

M Danilov, V Eiges, E Gershtein, Y Gershtcin, A Golutvin,

O I onkina, I Korolko, G Kostina, D Litvintsev, P Pakhlov,

S Semenov, A Snizhko, I Tikhomirov, Y Zaitscv

Accelerator DESY-DORIS-III Detector ARGUS

$\begin{array}{cc}\frac{\text { Reactions }}{e^{+} e^{-}} & 9.3-10.6 \mathrm{GcV}\left(\mathrm{E}_{\mathrm{cm}}\right) \\ e^{+} e^{-} \rightarrow \text { charm X } & " \\ e^{+} e^{-} \rightarrow \text { bottom X } & " \\ e^{+} e^{-} \rightarrow \Upsilon \text { (unspec) } & " \\ e^{+} e^{-} \rightarrow \text { hvy-lepton } \mathrm{x} & " \\ e^{+} e^{-} \rightarrow e^{+} e^{-\gamma \gamma} & \text { " }\end{array}$

Particles studied charm, bottom, $\Upsilon$ (unspec), hvy-lepton, $\nu_{r}$

Brief description Studies $b$ - and $c$-quark physics, the $\tau$ lepton and its neutrino, $\Upsilon$ spectroscopy, two-photon physics, fragmentation of quarks and gluons, and searches for new particles. The detector consists of a silicon strip detector and a microvertex drift chamber surrounding the beam pipe, a tracking chamber, shower and TOF counters, solenoid coils, and a large iron yoke. Upgraded in 1990/91. Data analysis in progress (July 96).

Journal papers NIM 163 (1979) 77, NIM 195 (1982) 475, NIM 205 (1983) 125, NIM 216 (1983) 35, NIM 217 (1983) 153, PL B134 (1984) 137, PL B135 (1984) 498, PL B146 (1984) 111, NIM A235 (1985) 26, NIM A237 (1985) 464, PL B150 (1985) 235 PL B153 (1985) 343, PL B154 (1985) 452, PL B156 (1985) 134, ZPHY C28 (1985) 45, PL B157 (1985) 326, PL B158 (1985) 525 , PL B160 (1985) 331, PL B162 (1985) 395, PL B163 (1985) 404, ZPHY C29 (1985) 167, NIM $A 249$ (1986) 277, NIM A252 (1986) 384, P'TE 2 (1986) 66, PRL 56 (1986) 549, PL B167 (1986) 360 , ZPHY C31 (1986) 181, PL B179 (1986) 398, PL B179 (1986) 403, PL B182 (1986) 95, ZPHY C33 (1986) 7, ZPHY C33 (1987) 359 , PL B185 (1987) 218, PL B185 (1987) 223, PL B185 (1987) 228, PL B187 (1987) 425, PL B 192 (1987) 245, PL B195 (1987) 102, PL B195 (1987) 307, PL B196 (1987) 101, PL B197 (1987) 452, PL B198 (1987) 255, PL B198 (1987) 577, ZPHY C35 (1987) 283, PL B199 (1987) 291, PL B199 (1987) 447, PL $\mathrm{B} 199$ (1987) 451, PL B199 (1987) 457, PL B199 (1987) $580, \mathrm{PL}$ B202 (1988) 149, ZPIYY C39 (1988) 177, PL B207 (1988) 109 PL B207 (1988) 349, PL B209 (1988) 119, PL B209 (1988) 380 , PL B210 (1988) 258, PL B210 (1988) 263, PL B210 (1988) 267, PL B210 (1988) 273, PL B211 (1988) 489, PL B212 (1988) 528, PI B215 (1988) 424, PL B215 (1988) 429, ZPHY C41 (1988) 1, ZPHY C41 (1988) 405, NIM A274 (1989) 189, NIM A275 (1989) 1, NIM A283 (1989) 544, NAT WISS 76 (1989) 52, ZPHY C41 (1989) 557, PL B217 (1989) 205, PL B219 (1989) 121, PL
B221 (1989) 422, ZPHY C42 (1989) 349, ZPHY C42 (1989) 519, ZPHY C42 (1989) 543, ZPHY C43 (1989) 45, ZPHY C43 (1989) 181, ZPHY C44 (1989) 547, PL B229 (1989) 175, PL B229 (1989) 304, PL B230 (1989) 162, PL B230 (1989) 169, PL B231 (1989) 208, PL B232 (1989) 398, PL B232 (1989) 554, MPL A5 (1990) 73, ZPHY A335 (1990) 231, ZPHY C46 (1990) 9, ZPHY C46 (1990) 15, ZPHY C48 (1990) 183, ZPHY C48 (1990) 543, PL B234 (1990) 409, PL B236 (1990) 102, PL B241 (1990) 278 , PL B245 (1990) 315, PL B246 (1990) 278, PL B247 (1990) 121, PL B249 (1990) 359, PL B250 (1990) 164, PL B254 (1991) 288 PL B255 (1991) 297, PL B255 (1991) 634, PL B260 (1991) 259, PL B262 (1991) 148, PL B267 (1991) 535, PL B269 (1991) 234, ZPHY C49 (1991) 349, ZPHY C50 (1991) 1, ZPHY C52 (1991) 353, PL B274 (1992) 239, PL B275 (1992) 195, PL B277 (1992) 209, PL B278 (1992) 202, PL B288 (1992) 367, PL B292 (1992) 221, PL B297 (1992) 425, ZPHY C53 (1992) 361, ZPHY C53 (1992) 367, ZPHY C54 (1992) 1, ZPHY C54 (1992) 13, ZPHY C55 (1992) 25, ZPHY C55 (1992) 179. ZPHY C55 (1992) 357, ZPHY C56 (1992) 1, ZPHY C56 (1992) 7, ZPHY C56 (1992) 339, MPL A8 (1993) 573, PL B303 (1993) 368, PL B308 (1993) $435, \mathrm{PL}$ B316 (1993) 608, PL B317 (1993) 227, PL B318 (1993) 397, ZPHY C57 (1993) 533, ZPHY C58 (1993) 61, ZPHY C58 (1993) 191, ZPHY C58 (1993) 199, ZPHY C60 (1993) 11, NIM A348 (1994) 465, PL B324 (1994) 249, PL B326 (1994) 320 PL B332 (1994) 451, PL B335 (1994) 526, PL B337 (1994) 383 , PL B338 (1994) 390, PL B340 (1994) 125, PL B340 (1994) 217, ZPHY C61 (1994) 1, ZPHY C62 (1994) 371, ZPHY C64 (1994) 375, PL B341 (1995) 441, PL B342 (1995) 397, PL B349 (1995) 576, PL B353 (1995) 554, ZPHY C65 (1995) 619, ZPHY C66 (1995) 63, ZPHY C68 (1995) 25, ZPHY C68 (1995) 215, NIM A373 (1996) 387, PL B374 (1996) 249, PL B374 (1996) 256, PL B374 (1996) 265, and ZPHY C69 (1996) 405.

Related experiments CESR-CLEO-II

E-mail contact f15sch $\Theta \times 4$ u2.desy.de

WWW Home-page

http://www.physik.uni-dortmund.de/ARGUS/argus.html

\section{DESY-HERA-HERMES}

(Proposed Jan 1990, Approved Oct 1992, Began data-taking 1995 In progress)

MEASUREMENT OF SPIN DEPENDENT STRUCTURE FUNCTIONS OF NUCLEONS

HERMES COLLABORATION

ALBERTA U - P Green, G Greeniaus, P Kitching, $K$ Martens

ARGONNE - O Hansen, H E Jackson, C E Jones, N Makins,

$\Upsilon$ O'Neill, D Potterveld, I' Zeuli

CAI TECH -. B Bray, P Carter, A Dvoredsky, B W Filippone,

S Jensen, W Korsch, K Mcllhany, R D McKeown, M Pitt

COLORADO U - J Brack, B Fox, E Kinney, D Mercer,

G Rakness, $R$ Ristinen, $R$ Smythe, $D$ van Westrum

DESY ... W Beckhusen, S Brons, M-A Funk, Y Holler, H Ihssen,

N Meyners, P Oelwein, H-J Plett, V Prahl, P Schuler, K Sinram,

M Spengos, G Woebke, K Woller, K Zapfe

DUBNA - A Fechtchenko, N D Gagunashvili, D Kirillov,

V Krivokhijin, V Mitsun, A P Nagaitsev, S Plyashkevich,

I Savin, G Yarygin

ERLANGEN U -. T Benisch, S Bernrenther, B Brann, M Dueren,

$M$ Ferstl, $K$ Fiedter, A Golendoukhin, C Grosshauser, A Gute,

N Koch, W Lachnit, F Neunreither, K Rith, E Steffens,

J Stenger, W Wander

FERRARA U - P Dalpiaz Ferretti, F Masoli, A Reali, F Sisini, P Slavich

FRASCATI - H Avakian, N Bianchi, G P Capitani, E De Sanctis.

P Di Nezza, A Fantoni, V Giordjian, R Mozzetti, V Muccifora, M Nupieri, A R Reolon-Cora, P Rossi

FREIBURG U -- M Beckmann, S Brauksiepe, H Fischer, J Franz, K Konigsmann, : $\mathrm{R}$ Ruh, $\mathrm{H}$ Schmitt, A Simon

GENT U - E Aschenaeur, D Ryckbosch, M Tytgat $R$ van de Vijver

HEIDELBERG, MAX PLANCK INST - A Borissov,

W Brueckner, A Bruell, P Geiger, W Hoprich, B Povh, E Volk

ILLINOIS U, URBANA - B Bains, R Cadman, H- Gao, R J Holt, M Miller, A Nathan, B Owen, S E Williamson

IIVERPOOL U - G R Court, J Morton, J Stewart, H Tallini

WISCONSIN U -- H J Bulten, W Haeberli, T Wise 


\section{SUMMARIES OF DESY EXPERIMENTS}

MIT, LNS - E Bruins, D DeSchepper, J Kelsey, L Kramer, J Martin, A Mateos, R Milner ( $\sqrt{ }$ Spokesperson), M Niczyporuk, $\mathrm{R}$ Redwine, T Shin, M Sutter, B Tipton

LEBEDEV INST - Y Bashmakov, E Devitsin, V Kozlov,

S Potashov, A Terkulov

MUNICH U, EXP PHYS - G Graw, R Fiertenberger, H Kolster, A Metz

NEW MEXICO STATE U - P Chumney, J Haas, G Kyle,

$\checkmark$ Papavassiliou, S Pate, G Schnell

NIKHEF, AMSTERDAM - J Blouw, $K$ de Jager,

$P$ de Witt Huberts, $M$ Doets, $T$ Henkes, $E$ Kok, $M$ Kolstein,

$\mathrm{H}$ R Poolman, $\mathrm{F}$ Udo, $\mathrm{R}$ van Bommel, J F J van den Brand, $G$ van der Steenhoven

OREGON STATE U - P Welch

PENN U - T Fortune, W Lorenzon, A Most, S Rudnitsky,

R Zurmuhie

ST PETERSBURG, INP - S L Belostotski, G Gavrilov, A Izotov,

A Y Kiselev, A Krivchitch, N Kuropatkin, S I Mannenkov,

$Y$ Naryshkin, V V Nelubin, V V Vikhrov

INFN, ROME - E Cisbani, S Frullani, F Garibaldi, M Iodice, G M Urciuoli

SIMON FRASER U \& TRIUMF - E Belz, P J Delheij, O Hausser R Henderson, $R$ Kaiser, C A Miller, R Openshaw, M C Vettcrli, $M$ Vincter

TOKYO U - H Ogami, Y Sakemi, T A Shibata

YEREVAN PHYS INST - A Airapertian, N Akopov, M Amarian, R. Avakian, A Avetissian, G Elbakian, V Garibyan, S Taroian, H Zohrabyan

DESY-IFH, ZEUTHEN - H Boettcher, S Brons, Y Gaerber,

D Hasch, V Korotkov, F Meissner, W-D Nowak, H Roloff,

A Schwind, U Stoesslein

Accelerator DESY-HERA Detector Spectrometer

Reactions Polarized target

$e^{-}$nucleon $\rightarrow e^{-X} \quad 27.5 \mathrm{GeV} / c\left(\mathrm{P}_{\mathrm{lab}}\right)$

Particles studied $p, n$

Brief description $T$ his is an internal target experiment in the

HERA electron storage ring. It measures both inclusive and semi-inclusive spin dependent deep inclastic scattering from the proton and neutron, and tests the fundamental Bjorken sum rule. Employs polarized internal gas targets of hydrogen, deuterium, and ${ }^{3}$ He. Internal targets have the advantage of being pure atomic species with no dilution factor. The angles and the energies of scattered particles are detcrmined by a magnetic spectrometer. Taking date (July 96).

Journal papers NIM A (in press).

Related experiments CERN-NA-047, SLAC-E-142, SLAC-E-143, SLAC-E-154

E-mail contact milner@axherl.desy.de

WWW Home-page http://dxhral.desy.de/

\section{DESY-HERA-H1}

(Proposed Jun 1985, Approved Jul 1986, Began data-taking May 1992 , In progress)

\section{H1: A DETECTOR FOR HERA}

\section{H1 COLLABORATION}

AACHEN, TECH HOCHSCH, I PHYS INST - C Berger,

W Braunschweig, H Genzel, 'T Hadig, M Hampel, H Itterbeck, C Keuker, T Koehler, H U Martyn, C Niedzballa, K Rabbertz, $K$ Rosenbauer

AACHEN, TECH HOCHSCH, III PHYS INST - H B Dreis,

G Fluegge, $H$ Graessler, H Kuester, P O Meyer, P Palmen,

H Pawletta, R Steinberg, W Struczinski, I Theissen, P Uelkes,

A Wagener, $M$ Wobisch

BIRMINGHAM U - J D Dowell, J Garvey, K Hewitt,

V L Hudgson, I R Kenyon, P R Newman, J P Sutton, L R West

BRUSSELS U, IIHE - M Barth, G Bertrand-Coremans,

A Buniatian, B Clerbaux, E A De Wolf, D P Johnson,

P Marage, A Panitch, R Roosen, P Van Esch, P Van Mechelen

CRACOW - L Goerlich, K Golec-Bicrnat, L Hajduk, J Martyniak,

S Mikocki, G Nowak, K Rybicki, J Turnau

UC, DAVIS -- W Dlugosz, G Pope, F Rouse, S Willard
DESY - R Barschke, W Bartel, H J Behrend, R Beyer,

R Buchholz, J Buerger, A J Campbell, M Charlet, A DeRoeck, G Eckerlin, E Elsen ( $\sqrt{ }$ Deputy Spokesperson), $R$ Felst, K Flamm, G Franke, J Gayler, R Gerhards, D Haidt, D Mofimann, M Kander, M Kausch, S Kazarian, C Kleinwort, G Knies, V Korbel, H Krehbiel, F Lehner, F Linsel, B List, A Meyer, J Meyer, G Mueller, C Niebuhr, J E Olsson, E Panaro, S Prell, S Reinshagen, A Schoening, V Schroeder, V Shekelyan, P Steffen, 3 Stier, E Tzamariudaki, T Wilksen, G G Winter, E Wuensch

DORTMUND U - K Borras, J G Contreras, M Dirkmann. M Fleischer, D Goldner, M Hopepner, M Huette, T Kraemerkaemper, J Kurzhoefer, D Lueke, H Rick, J Spiekermann, K Wacker, D Wegener

ECOLE POLYTECHNIQUE - B Andrieu, V Boudry, S Levonian, A Migliori, F Moreau, Y Sirois, A Specka, D Vandenplas

GLASGOW U - I O Skillicorn

HAMBURG U - S Aid, V Blobel, L Buengener, F W Buesser, A B Fahr, B Heinemann, G Heinzelmann, G Lindstroem, J Lipinski, G C Lopez, G Martin, A Meyer, B Naroska,

F Niebergall, S Riess, S Schiek, G Schmidt, H Spitzer, J Steinhart, G Weber, C Wittek

HEIDELBERG, MAX PLANCK INST - M Beck, W Brueckner

'T Haller, D M Jansen, L Lytkin, F Metlica, T Nunnemann, $B$ Povh, R Todenhagen

HEIDELBERG U, PHYS INST - A Braemer, F Eisele, M Erdmann, I Katzy, O Kaufmann, P Schleper, B Schwab, U Straumann, $T$ Wengier, $M$ Werner

HEIDELBERG U, IHEP - C Brune, J Janoth, K Meier, S Schleif, $\Lambda$ Stellberger, J Stiewe, S Tapprogge, $M$ Weber

MOSCOW, IT'EP - A Babaev, A Droutskoi, V Efremenko,

A Fedotov, I Gorelov, V Lubimov, V Nagovizin, D Ozerov,

A Rostovtsev, $A$ Semenov, $V$ Solochenko, $V$ Tchernyshov, $S$ Tchetchelnitski, A Zhokin

KIEL U - W D Dau, C Gruber, U Kathage, G Siegmon, U Siewert KOSICE, IEF - J Ban, D Bruncko, J Ferencei, R Maracek, $P$ Murin

LANCASTER U - S Burke, A B Clegg, C L Davis, P Dixon,

$\mathrm{R} C \mathrm{C}$ Henderson, $\mathrm{D}$ Newton

LIVERPOOL U - S Cocks, C Cormack, J B Dainton, 'T R Ebert,

E Gabathuler, T' Greenshaw, T Holtom, R Martin, S J Maxfield, S J McMahon, D Milstead, M Oakden, G D Patel

LEBEDEV INST - V Andreev, P Baranov, A Belousov, A Fomenko, N Gogitidze, S K Kotelnikov, A Lebedev, E Malinovski, S Rusakov, I Sheviakov, L N Shtarkov, P Smirnov, Y Soloviev, A Usik, Y Vazdik

LUND U - L Joensson, H Jung, H Kuester, M Lindstroem MANCHESTER U -- M Anderson, P Biddulph, P Bispham, M J Burton, J M Foster, C D Hilton, M Ibbotson, S D Kolya, J W Lomas, R Marshall, B Waugh

MARSEILLE U, LUMINY - D Calvet, M C Cousinou,

S Kermiche, J Marks, I Negri, A Pieuchot, C Vallee

MUNICH, MAX PLANCK INST - T Carli, M Flieser, F Gaede. G Grindhammer, A Gruber, M F Hess, C Kiesling, J Koehne, D Kruecker, M Kuhlen, J Moeck, H Oberlack, K Rueter, P Schacht, E Schuhmann, A Wegner

ORSAY, LAL - Y Ban, J C Bizot, V Brisson, A Courau, B Delcourt, L Favart, A Jacholkowska, M Jaffre, G Lobo, C Pascaud, F Squinabol, $Z$ Zhang, F Zomer

PARIS, CURIE UNIV VI \& PARIS, UNIV VII, LPNHE E Barrelet, U Bassler, G Bernardi, S Dagoret, M Goldberg, B Gonzalcz-Pineiro, M W Krasny, H K Nguyen, P Zini PRAGUE, INST PHYS - J Cvach, I Herynck, J Hladky,

$P$ Reimer, M Tasevsky

CHARLES U -- J Formanek, S Valkar, A Valkarova, J Zacek PSI, VILLIGEN - K Gabathuler, R Horisberger, M Wagener QUEEN MARY - WESTFIELD COLL - K T Donovan, E Eisenhandler, R K Griffiths, P I P Kalmus, D Kant, M P J Landon, T Mavroidis, E Rizvi, R Rylko, G Thompson. $W$ von Schlippe

RUTHERFORD - D Clarke, J A Coughlan, D G Cussans, W J Haynes, A Mehta, J V Morris, D P C Sankey ROME U \& INFN, ROME - P Di Nezza, F Ferrarotto, B Stella SACLAY - G Cozzika, M David, J Feltesse, B Laforge, E Perez, C Royon, P Verrecchia, G Villet, J Zsembery

WUPPERTAL U - K Daum, J Ebert, N Magnussen, J Martens, H Meyer, D Schmidt

DESY-IFH, ZEUTHEN -- J Baehr, H Ehrlichmann, M Gebauer, A Glazov, H Henschel. K H Killer, H H Kaufmann, M Klein, 


\section{SUMMARIES OF DESY EXPERIMENTS}

H Kolanoski, P Kostka, T Kurca, W Lange, R Nahnhauer, $T$ Naumann, E Peppel, U Stoesslein, K Stolze, $M$ Winde ZURICH U - H P Beck, C Dollfus, S Egli, D Mueller, P Robmann, F Sefkow, $P$ Trueol, M zurNedden

ZURICH, ETH - R A Eichler ( $\sqrt{ }$ Spokesperson), C Grab,

U Langenegger, $\vec{H}$ Niggli, D Pitzl, G Tsipolitis

Accelerator DESY-HERA Detector H1

Reactions

$e^{-} p$

Brief description Measures energy and direction of electrons photons, and particle jets. Identifies leptons by the shower shape and neutrinos by precise missing energy measurements. The detector consists of a large superconducting solenoid with tracking chambers and a liquid argon calorimeter inside. An additional iron absorber instrumented with streamer tubes is outside the solenoidal coil. In 1995, the backward (electron) direction EM calorimeter was replaced by a SpaCal-type electromagnetic + hadronic calorimeter, and Roman pots were added in the forward (proton) direction to measure elastically scattered protons. In 1996, silicon vertex detectors were added in the barrel (CST) and backward (BST) directions, and a SpaCal-type neutron counter installed in the forward direction. Taking data (July 96).

Journal papers NIM A240 (1985) 63, NIM A253 (1987) 467, NIM A257 (1987) 479, NLM A265 (1988) 419, NIM A269 (1988) 560, NIM A270 (1988) 334, IEEE TNS 36 (1989) 331, NIM A275 (1989) 197, NIM A275 (1989) 246, NIM A277 (1989) 368, NIM A279 (1989) 57, NIM A279 (1989) 217, NIM A283 (1989) 375 , NIM A283 (1989) 467, NIM A283 (1989) 471, NIM A283 (1989) 487, NIM A283 (1989) 537, NIM A283 (1989) 622, NP (PROC SUPPL) 16 (1989) 518, NIM A289 (1990) 446, NIM A302 (1991) 277 , NIM A310 (1991) 535, NLM A312 (1992) 457, NIM A323 (1992) 184, NIM A323 (1992) 401, NIM A323 (1992) 532, NIM A323 (1992) 537, NIM A336 (1993) 460, NIM A336 (1993) 499, PL B297 (1992) 205, NIM A336 (1993) 460, NIM A336 (1993) 499, PL B298 (1993) 469, PL B299 (1993) 374, PL B299 (1993) 385 , PL B314 (1993) 436, NP B396 (1993) 3, NP B407 (1993) 515, NIM A344 (1994) 492, NIM A350 (1994) 57, PL B321 (1994) 161, PL B324 (1994) 241, PL B328 (1994) 176, PL B338 (1994) 507, PL B340 (1994) 205, APP B25 (1994) 319, APP B25 (1994) 1883 , NC $107 \mathrm{~A}$ (1994) 2109 , NC $107 \mathrm{~A}$ (1994) 2345 , ZPHY C61 (1994) 59, ZPHY C63 (1994) 377, ZPHY C64 (1994) 545, NP B429 (1994) 477, PL B346 (1995) 415, PL B348 (1995) 681, PL B353 (1995) 578, PL B354 (1995) 494, PL B356 (1995) 118 , PL B358 (1995) 412, ZPHY C66 (1995) 529, ZPHY C67 (1995) 565, ZPHY C69 (1995) 27, NP B435 (1995) 3, NP B439 (1995) 471, NP B445 (1995) 3, NP B445 (1995) 195, NP B449 (1995) 3, NIM A372 (1996) 188, NIM A372 (1996) 399, NIM A374 (1996) 149, PL B369 (1996) 173, PL B279 (1996) 319, ZPHY C70 (1996) 17, ZPHX C70 (1996) 609, NP B463 (1996)

3 , NP B468 (1996) 3, and NP B470 (1996) 3 .

Related experiments DESY-HERA-ZEUS

E-mail contact eichler@particle.phys.ethz.ch, elsen@dice2.desy.de WWW Home-page http://dice2.desy.de/

\section{DESY-HERA-ZEUS}

(Proposed Jun 1985, Mar 1986, Approved Nov 1986, Began datataking May 1992, In progress)

\section{ZEUS: A DETECTOR FOR HERA}

ZEUS COLLABORATION

ARGONNE - M Derrick, D Krakauer, S Magill, D Mikunas,

B Musgrave, J R Okrasinski, J Repond, R Stanek, R L Talaga, H Zhang

ANDREWS U - M C K Mattingly

BOLOGNA U \& INFN, BOLOGNA - P Antonioli, G Bari,

$M$ Basile, $\mathrm{L}$ Bellagamba, D Boscherini, A Bruni, G Bruni,

P Bruni, G Cara Romeo, G Castellini, L Cifarelli, F Cindolo,

A Contin, M Corradi, I Gialas, P Giusti, G Iacobucci,

G Laurenti, G Levi, $\Lambda$ Margotti, T Massam, R Nania,

F Palmonari, A Pesci, A Polini, G Sartorelli, Y Zamora Garcia.

A Zichichi

BONN U - C Amelung, A Bornheim, J Crittenden, R Deffner,

$T$ Doeker, M Eckert, L Feld, A Frey, M Geerts, M Grothe,
H Hartmann, K Heinloth, L Heinz, E Hilger, H P Jakob, U F Katz, S Mengel, E Paul, M Pfeiffer, C Rembser,

D Schramm, J Stamm, R Wedemeyer

BRISTOL U - S Campbell-Robson, A Cassidy, W N Cottingham,

$N$ Dyce, B Foster, S George, M E Hayes, G P Heath, H F Heath,

D Piccioni, D G Roff, R J Tapper, R Yoshida

CALABRIA U \& INFN, COSENZA - M Arneodo, R Ayad,

$M$ Capua, A Garfagnini, L Iannotti, M Schioppa, G Susinno

NEVIS LABS, COLUMBIA $U-$ A Caldwell ( $\checkmark$ Deputy

Spokesperson), N Cartiglia, Z, Jing, W Liu, J A Parsons, S Ritz,

F Sciulli, P B Straub, L Wai, S Yang, Q Zhu

CRACOW - P Borzemski, J Chwastowski, A Eskreys,

Z Jacubowski, M B Przybycien, M Zachara, L Zawiejski

CRACOW, INST PHYS NUCL TECH - 2 Adamczyk,

B Bednarck, K Jelen, D Kisielewska, T Kowalski, M Przybycien, E Rulikowska-Zarebska, L Suszycki, J Zajac

JAGELLONIAN U - Z Dulinski, A Kotanski

DESY - G Abbiendi, L A T Bauerdick, U Behrens, $H$ Beier,

J K Bienlein, G Cases, O Deppe, K Desler, G Drews,

$M$ Flasinski, D J Gilkinson, C Glasman, P Gottlicher, J GrosseKnetter, T Haas, W Hain, D Hasell, $\mathrm{H}$ Hessling, Y Iga,

K Johnson, P Joos, M Kasemann, R Klanner ( $\checkmark$ Spokesperson),

W Koch, U Kotz, H Kowalski, J Labs, A Ladage, B Lohr,

M Lowe, D Luke, J Mainusch, O Manczak, J Milewski,

T Monteiro, J S T Ng, D Notz, K Ohrenberg, K Piotrzkowski,

M Roco, M Rohde, J Roldan, U Schneekloth, W Schulz,

F Selonke, B Surrow, E Tassi, T Voss, D Westphal, G Wolf,

U Wollmer, C Youngman, W Zeuner

DESY-IFH, ZEUTHEN - H J Grabosch, A Kharchilava,

S M Mari, A Meyer, S Schlenstedt, N Wulff

FLORENCE U \& INFN, FLORENCE - G Barbagli, E Gallo,

$P$ Pelfer

FRASCATI - S De Pasquale, G Maccarrone, L Votano

FREIBURG U - A Bamberger, S Eisenhardt, T Trefzger, $S$ Wolfle

GLASGOW U - J T Bromley, N H Brook, P J Bussey, A T Doyle,

D H Saxon, L E Sinclair, M L Utley, A S Wilson

HAMBURG U - B D Burow, A Dannemann, L Hagge, U Holm,

D Horstmann, E Lohrmann, G Poelz, W Schott, R Sinkus,

K Wick, F Zetsche

IMPERIAL COLL - T C Bacon, N Brummer, I Butterworth, V \& Harris, G Howell, B Y H Hung, L Lamberti, K R Long,

D B Miller, $N$ Pavel, A Prinias, $3 \mathrm{~K}$ Sedgbeer, D Sideris, A Whitfield

IOWA U -. U Mallik, M Z Wang, S M Wang, J T Wu

JULICH, FORSCHUNGSZENTRUM - P Cloth, D Filges

KOREA U - S H An, G H Cho, B J Ko, S B Lee, S W Nam,

H S Park, S K Park

LOUISIANA ST'ATE U - S Kartik, H J Kim, R R McNeil, W Metcalf, V K Nadendla

MADRID, AUTONOMA U - F Barreiro, I F de 'Iroconiz

J del Peso, J P Fernandez, R Graciani, J M Hernandez,

L Hervas, L Labarga, M Martinez, J Puga, J Terron

MCGILL U - F Corriveau, D S Hanna, J Hartmann, L W Hung,

I N Lim, C Matthews, P M Patel, M Riveline, M St Laurent,

D G Stairs, R Ullmann, G Zacek

MEIJI GAKUIN U - T Tsurugai

MOSCOW PHYS ENG INST - V Bashkirov, B A Dolgoshein,

A Stifutkin

MOSCOW STATE U - G L Bashindzhagian, P F Ermolov, L K Gladilin, Y A Golubkov, V D Kobrin, I A Korzhavina,

V A Kuzmin, O Y Lukina, A S Proskuryakov, A A Savis,

L M Shcheglova, A N Solomin, N P Zotov

NIKHEF, AMSTERDAM \& AMSTERDAM U - M Botje,

$F$ Chlebana, $M$ de Kamps, $E$ de Wolf, J Engelen, $P$ Kooijman,

A Kruse, H Ticcke, A Van Sighem, $R$ van Woudenberg,

W Verkerke, J Vossebeld, M Vreeswijk, L Wigrers

OHIO S'IATE U - D Acosta, B Bylsma, L S Durkin, J Gilmore,

C Li, $T$ Y Ling, P Nylander, I H Park, T A Romanowski

OXFORD U - D Bailey, R J Cashmore, A M Cooper-Sarkar,

R C E Devenish, N Harnew, M. Lancaster, L Lindemann,

$J$ McFall, $C$ Nath, $V A$ Noyes, $\Lambda$ Quadt, $J \mathrm{R}$ Tickner,

H Uijterwaal, R Walczak, D S Waters, F F Wilson, 'T Yip

PADUA U \& INFN, PADUA - A Bertolin, R Brugnera,

R Carlin, F Dal Corso, M De Giorgi, U Dosselli, S Limentani,

M Morandin, M Posocco, L Stanco, R Stroili, C Voci, F Zuin

PENN STATE U - J Bulmahn, R G Feild, B Y Oh, J Whitmore ROME U \& INFN, ROME - G D'Agostini, G Marini, A Nigro RUTHERFORD - J C Hart, N A McCubbin, 'T P Shah 


\section{SUMMARIES OF DESY EXPERIMENTS}

UC, SANTA CRUZ - E Barberis, T Dubbs, C Heusch, W Lockman, J T Rahn, H F Sadrozinski, A Seiden, $\mathrm{M}$ Van Flook, D C Williams

SIEGEN U - J Biltzinger, O Schwarzer, R J Seifert, A H Walenta TEL AVIV U - H Abramowicz, G Briskin, S Dagan, A Levy

TOKYO U, INS - J I Fleck, M Inuzuka, T Ishii, M Kuze, S Mine, M Nakao, I Suzuki, K Tokushuku, K Umemori, S Yamada, Y Yamazaki

TOKYO METROPOLITAN U - M Chiba, R Hamatsu, T Hirose, K Homma, S Kitamura, T Matsushita, K Yamauchi

TURIN U \& INFN, TURIN - R Cirio, M Costa, M I Ferrero,

$S$ Maselli, C Peroni, $R$ Sacchi, $\Lambda$ Solano, A Staiano

TURIN U, ALESSANDRIA \& INEN, TURIN - M Dardo

TORONIO U - D C Bailey, F Benard, M Brkic, C P Fagerstrom, G F Hartner, K K Joo, G M Levman, J F Martin, R S Orr, S Polenz, C R Sampson, D Simmons, R J Teuscher

UNIVERSITY COLL, LONDON - J M Butterworth,

C D Catterall, T W Jones, P B Kaziewicz, J B Lane.

R L Saunders, J Shulman, M R Sutton

VIRGINIA TECH - B Lu, L W Mo

WARSAW U, IEP - W Bogusz, J Ciborowski, J Gajewski,

G Grzelak, M Kasprzak, M Krzyzanowski, K Muchorowski,

R J Nowak, J M Pawlak, 'T Tymieniecka, A K Wroblewski,

I A Zakrzewski, A F Zarnecki

WARSAW, INST NUCL STUDIES - M Adamus

WEIZMANN INST - C Coldewey, Y Eisenberg, D Hochman, U Karshon, D Revel, D Zer-Zion

WISCONSIN U - W F Badgett, J Breitweg, D Chapin, R Cross,

S Dasu, C Foudas, R J Loveless, S Mattingly, D D Reeder,

S Silverstein, W H Smith, A Vaiciulis, $M$ Wodarczyk

YORK U, CANADA - S Bhadra, M L Cardy, W R Frisken,

M Khakzad, W N Murray, W B Schmidke

Accelerator DESY-HERA Detector ZEUS

\section{Reactions}

$$
\begin{array}{cc}
e^{-} p & 300 \mathrm{GeV}\left(\mathrm{E}_{\mathrm{Cm}}\right) \\
e^{+} p & "
\end{array}
$$

Particles studied leptons, $p, K^{0}, \Lambda$, strange, $\rho^{0}, \omega, \phi, J / \psi(1 S)$, vmeson, charm

Brief description Measures neutral and charged current processes in electron-proton and positron-proton interactions $(27.5 \mathrm{GeV}$ on $820 \mathrm{GeV})$ and searches for now interactions and new particles. The detector emphasis is on accurate identification and measurement of jets and leptons. The main detector components are a high-resolution compensating uraniumscintillator calorimeter and a central tracking detector surrounded by a superconducting coil. An instrumented iron absorber catches the tail of hadronic showers and identifies muons. A large fraction of the solid angle is further covered by muon detectors. Bending magnets of the machine are used as spectrometers for forward scattered protons. Additional calorimeters measure forward neutron production and tag electrons and positrons scattered with small transverse momentum. Taking data (July 96).

Journal papers IEEE TNS 36 (1989) 465, NIM A274 (1989) 134 NIM A289 (1990) 115, NIM A290 (1990) 95, NIM A292 (1990) 259, NIM A300 (1991) 480, NIM A306 (1991) 485, NIM A309 (1991) 101, NIM A313 (1992) 126, NIM A321 (1992) 356, PL B297 (1992) 404, PL B293 (1992) 465, NIM A333 (1993) 342 NIM A336 (1993) 23, PL B303 (1993) 183, PL B306 (1993) 158 , PL B306 (1993) 173, PL B315 (1993) 481, PL B316 (1993) 207, PL B316 (1993) 412, ZPHY C59 (1993) 231, NLM A338 (1994) 254, NIM A342 (1994) 260, PL B322 (1994) 287, PL B332 (1994) 228, PL B338 (1994) 483, NC $107 \mathrm{~A}$ (1994) 2123 ZPHY C63 (1994) 391, PL B342 (1995) 417, PL B345 (1995) 576 , PL B346 (1995) 399, PC B348 (1995) 665, PL B349 (1995) 225, PL B350 (1995) 120, PL B354 (1995) 163, PL B356 (1995) 129 , PL B356 (1995) 601, PL B363 (1995) 201, PRL 75 (1995) 1006, ZPHY C65 (1995) 379, ZPHY C65 (1995) 627, ZPHY C67 (1995) 81, ZPHY C67 (1995) 93, ZPHY C67 (1995) 227, ZPHY C68 (1995) 29, ZPHY C68 (1995) 113, ZPHY C68 (1995) 569 ZPHX C69 (1995) 39, PL B369 (1996) 55, ZPHX C69 (1996) 607, ZPYY C70 (1996) 1, and ZPHY C70 (1996) 391.

Related experiments DESY-HERA-H1

E-mail contact klanner@desy.de, caldwell@vxdesy.desy.de WWW Home-page http://zow00.desy.de:8000/

\section{DESY-HERA-B}

(Proposed May 1994, Approved Jun 1994, Feb 1995, In preparation)

\section{AN EXPERIMENT TO STUDY $C P$ VIOLATION IN THE} $B$ SYSTEM USING AN INTERNAL TARGET AT THE HERA PROTON RING

\section{HERA-B COLLABORATION}

Accelerator DESY-HERA Detector Spectrometer

Brief description The main goal is to measure the violation of $C P$ symmetry in the decay channel $B \rightarrow K_{S}^{0} J / \psi$. Uses the $820 \mathrm{GeV}$ HERA proton ring and an internal fixed target. 'The target consists of a set of movable wires which act like collimators in the halo of the proton beam. The detector provides a good $B$ decay vertex resolution, lepton and kaon identification, and multiple events reconstruction per bunch crossing. A $2 \mathrm{~m}$ long vertex detector consists of layers of doublesided silicon with perpendicular orientation to the beam. It is followed by tracking chambers inside the spectrometer magnet which provides a field integral of $2.2 \mathrm{Tm}$, by RICH and TRD counters for particle identification, and by the electromagnetic calorimeter and the muon system. The collaboration consists of about 240 physicists from thirteen countries and more than 30 institutions. Expected to begin data taking in June 1998. For more information, please contact the Spokesperson, Dr. Andreas Schwarz [DESY]

Related experiments CERN-WA-089, FNAL-771, FNAL-789

E-mail contact schwarza@hera-b.desy.de

WWW Home-page http://www-hera-b.desy.de/

\section{DESY Future Plans}

The HERA accelerator experiments remain in the focus of particie physics research at DESY in the coming years. H1 and ZEUS, the two collision experiments using the $27-\mathrm{GcV}$ electron/positron beam and $820-\mathrm{GeV}$ proton beam will continue to study the proton structure functions at very low $x$, test the QCD and electroweak interactions and search for new phenomena. HERMES, which uses a longitudinally polarized electron/positron beam and a polarized nucleon gas target, will keep investigating the origin of the nucleon spin. The second beam-target experiment, HERA-B, which is scheduled to begin the study of $C P$ violation in the $B$ meson system in 1998 , will use the proton beam and an internal aluminum wire target. In machine physics, the efforts are focused on the Next Accelerator project, an electron-positron linear collider at $500 \mathrm{GeV}$ c.m. International collaborations based at DESY are studying two possible paths: One is the $\mathbf{S - B a n d}$ Lincar Collider (SBLC) which uses normally-conducting copper resonators operating in the frequency region of $3 \mathrm{GHz}$, and the other is the TeV-Energy Superconducting Linear Accelerator (TESLA) with superconducting accelerating structures operating in the frequency region of $1.3 \mathrm{GHz}$. TESLA is the only linear collider project worldwide which uses the integrated $X$-ray lasers. Test facilities for both projects are under construction at DESY. 


\section{SUMMARIES OF FERMILAB EXPERIMENTS}

\section{Selected FNAL Experiments}

\section{FNAL-665}

(Proposed Oct 1980, Approved Jul 1981, Jan 1989, Began datataking 1987, Completed data-taking Jan 1992)

\section{MUON SCATTERING WITH HADRON DETECTION} AT THE TEVATRON

FREIBURG U - T Dreyer, M Erdmann, 3 Haas, M Lenski, W Mohr, G Seigert, H E Stier, M O Wilhelm

ARGONNE - D F Geesaman, R Gilman, M C Green,

H E Jackson, S Kaufman, E R Kinney, $x$ B W Kirk

D H Potterveld. S Tentindo-Repond, H J Trost, A Zghiche

UC, SAN DIEGO - R D Kennedy, H G E Kobrak. P Madden, $J$ R Ostrick, A Salvarani, R A Swanson

FERMILAB - B R Baller, G B Coutrakon, J E Hanlon,

S Krzywdzinski, H Melanson, H E Montgomery, J G Morfin, C Salgado, $S$ A Wolbers

HARVARD U -. J M Conrad, G Y Fang, A V Kotwal,

D G Michael, R B Nickerson, F M Pipkin, M H Schmitt, $\mathrm{R}$ Wilson

ILLINOIS U, CHICAGO - M R Adams, D A Averill, $T \mathrm{~J}$ Carroll, R S Guo, C Halliwell, D E Jaffe, S R Magill, D W Mcleod, T McKibben

CRACOW - A Eskreys, J Figiel, P Malecki, K Olkiewicz, B Pawlik, P Stopa

CRACOW, INST PHYS NUCL TECH - K Dziunikowska LIVERMORE - P L Anthony, F S Dietrich

MARYLAND U . S Aid, S Kunori, S C O'Day, E J Ramberg, A Skuja, G A Snow, P H Steinberg, R Talaga

MI'I - M Baker, W Busza, L S Osborne, J J Ryan

MUNICH, MAX PLANCK INST - M Aderholz, F Botterveck, I Derado. V Eckardt, H J Gebauer, D Hantke, G Jancso, K Kadija, N Koschorz, A S Manz, P Mooshofer, N Schmitz, H J Seyerlein, S Soldner-Rembold, M Vidal, W Wittek

NORTHWESTERN U - II M Scheliman ( $\checkmark$ Spokesperson), P Spentzouris

OIIIO U - H L Clark, R W Finlay, K H Hicks

PENN U - A Banerjee, K Griffioen

WASHINGTON U, SEATTLE - A A Bhatti, U Bratzler,

R Davisson, W M Dougherty, D M Jansen, Z Jin, J J Lord,

H J Lubatti, M J Mitchell, R S Perry, B Venema, R J Wilkes, T C Zhao

WUPPERTAL U -. H M Braun, H Breidung, U Ecker, R Otten, A Roeser

YALE U - S K Dhawan, V W Hughes, V Papavassiliou, K P Schueler, H Venkataramania

Accelerator FNAL-TEV Detector CCM

\begin{tabular}{l} 
Reactions \\
\hline muon $e^{-} \rightarrow$ Polarized beam $e^{-}$
\end{tabular}

muon nucleus $\rightarrow$ muon $\phi X$
Brief descraption Studies (1) the properties of hadron systcms

recoiling from deep inelastic muon collisions, (2) the nucleon structure functions, and (3) exclusive vector meson production. Uses the supcrconducting vertex magnet from CERN. The first run was completed in 1988 , the second run, with a number of different targets $\left(\mathrm{H}_{2}, \mathrm{D}_{2}, \mathrm{C}, \mathrm{Ca}, \mathrm{Xe}\right.$, and $\left.\mathrm{Pb}\right)$ and an upgrade of the vertex spectrometer tracking system, in 1990/91. Data analysis in progress (July 96)

Journal papers IEEE TNS 33 (1986) 205, NIM A291 (1990) 533, PL B272 (1991) 163, PL B287 (1992) 375, PRL 68 (1992) 3266 PRL 69 (1992) 1026, PL B308 (1993) 418, PL B309 (1993) 477, PR D48 (1993) 5057, PRL 72 (1994) 466, PL B335 (1994) 535,
ZPHY C61 (1994) 179, ZPHY C61 (1994) 539, PR D50 (1994) 1836, PRL 74 (1995) 1525, PRL 74 (1995) 5198, PRL 75 (1995) 1466, ZPHY C65 (1995) 225, ZPIYY C67 (1995) 403, ZPHY C71 (1996) 391, and PR D54 (1996) (in print).

Related experiments FNAL-098

E-mail contact schellman@fnal.gov

$W W W$ Home-page http://fnmux4.fnal.gov/

\section{FNAL-672A}

(Proposed Feb 1981, Approved Jul 1981, Began data-taking 1987, Completed data-taking Jan 1992)

\section{A STUDY OF HADRONIC FINAL STATES PRODUCED} IN ASSOCIATION WITH HIGH-MASS DIMUONS

FERMILAB - J C Krider

ILLINOIS U, CHICAGO - H S Goldberg, R L Jesik, H Mendez, J Solomon, F Vaca

INDIANA U - R R Crittenden, A R Dzierba, A M Gribushin,

S Kartik, J Li, R Li, T R Marshall, H J Martin, P T Smith,

T Sulanke, A Zieminski (Spokesperson)

LOUISVILLE U - C L R Davis

MICHIGAN U, FLINT - L J Dauwe

SERPUKHOV - V V Abramov, Y M Antipov, B Baldin,

S P Denisov, A Dyshkant, V Glebov, Y Gorin, V I Koreshev,

A Krinitsyn, A A Petrukhin, V Podstavkov, V I Sirotenko,

R Sulayev

Accelerator FNAL-TEV Detector Spectrometer

Reactions

$p$ nucleus $\rightarrow \mu^{+} \mu^{-} \mathrm{X} \quad 500,800 \mathrm{GeV} / c$

$\pi^{-}$nucleus $\rightarrow \mu^{+} \mu^{-} \mathrm{X} \quad 500 \mathrm{GcV} / \mathrm{c}$

Particles studied $J / \psi(1 S), \psi(2 S), \chi_{c 1}(1 P), \chi_{c 2}(1 P), \rho, \omega, \phi$, bottom

Brief description Studies particles produced in association with vector mesons (including $\mathrm{J} / \psi$ ) and high mass dimuons. Ran with $\mathrm{H}, \mathrm{Be}$ and $\mathrm{Cu}$ targets. Collected approximately $2 \mathrm{M}$ fully linked dimuon events (over $30 \mathrm{~K} \psi$ 's) with different beams. Uses E672/E706 spectrometer. Data analysis in progress (May 96).

Journal papers NIM A270 (1988) 99, PR D41 (1990) 1, PRL 74 (1995) 495, and PR D53 (1996) 4723

E-mail contact zieminski@fnal.gov, zieminski@ind.physics.indiana.edu

\section{FNAL-687}

(Proposed Jan 1981, Approved Dec 1983, Began data-taking 1987, Completed data-taking Jan 1992)

HIGH-ENERGY PHOTOPRODUCTION OF STATES CONTAINING HEAVY QUARKS AND OTHER RARE PHENOMENA

\section{E687 COLLABORATION}

BOLOGNA U -. V Giordano, G Molinari

COLORADO U - C W Bogart, H W K Cheung, P Coteus,

SW Culy, J P Cumalat ( $\checkmark$ Spokesperson), C J Dallapiccola,

D P Edmonds, J Ginkel, V Greene, G Introzzi, W E Johns,

R L Ladbury, M Nehring, G E Schultz

FERMILAB - M E Binkley, J N Butler ( $\checkmark$ Spokesperson),

S Cihangir, I Gaines, P H Garbincius, L Garren, M F Gormley,

S A Gourlay, D J Harding, P H Kasper, A $E$ Kreymer,

P L G Lebrun, J Peoples, Jr, S Shukla, J T Volk

FRASCATI .. S Bianco, S Camponeschi, F Celani, G Digiovanni,

M Enorini, F Fabbri, G Ferretti, M Giardoni, G Giraudo,

A Maccari, L Passamonti, D Riondino, G Rivellini, V Russo,

S Sarwar, A Scotti Di Uccio, A Zallo

ILLINOIS U, URBANA - F D Cogswell, R L Culbertson,

R W Gardner, R Greenc, G R Jaross, 'T Kroc, K L Lingel,

'T A O'Halloran, Jr. T Protzman, H G Scott, P D Sheldon,

$\checkmark \mathrm{J}$ Simaitis, $T$ E Trumpinski, J R Wilson, J E Wiss

KOREA U - B G Cheon, J S Kang, K Y Kim

MYAAN U \& INFN, MILAN - G Alimonti, G Bacchiocchi,

G Bellini, L Bodini, D Brambilla, B Caccianiga, W R Cavaletti, 


\section{SUMMARIES OF FERMILAB EXPERIMENTS}

L Cinquini, P D'Angelo, M Di Corato, P A Falbo, P Frabetti M G Giammarchi, S Grabar, D Hazan, P Inzani, F Leveraro, E Macavero, S Malvezzi, P F Manfredi, G Massimiliano, M Mazzanti, D Menasce, S Meneghini, E Meroni, L Moroni, S Moroni, G Oriani, F Palombo, D Pedrini, L Perasso, F Ragusa, A Sala-Grabar, S Sala, M Szaswlowski, D Torretta, $M$ Vittone, F Zuffa

NORTHWESTERN U - M Artuso, T N Boulos, D A Buchholz, D R Claes, B Gobbi, B O'Reilly, S-W Park, R A Schluter. $R$ Yoshida

NOTRE DAME U - J M Bishop, N N Biswas, J K Busenitz, N M Cason, J D Cunningham, S Grenquist, C J Kennedy, V P Kenney, G N Kim, T F Lin, J Maier, E Mannel, A P Mcmanus, R J Mountain, D L Puseljic, R C Ruchti, W D Shephard, J A Swiatek, P Wilkins, $Z$ Y Wu, M E Zanabria

PAVIA U - V Arena, G Belli, G Boca, C Castoldi, S Cerlesi, $R$ Diaferia, G Eerretti, C Fontana, G Fontana, G Gianini,

S Rahti, S P Ratti, S Rescia, C Riccardi, V Speziali, P Trespi.

L Viola, $P$ Vitulo

UC, DAVIS - G P Grim, V Paolone, P M Yager

PUERTO RICO U, RIO PIEDRAS - A Lopez, L Mendez,

IC Palathingal

MEXICO, IPN - H Mendez

NORTH CAROLINA U - T F Davenport

TENNESSEE U - G Blackett, W Bugg, K Danyo, T Handler,

$\mathrm{G}$ Kondo, $\mathrm{M}$ Phisharody

Accelerator FNAL-TEV Detector Spectrometer

\section{Reactions}

$\gamma$ nucleus $\rightarrow \mathrm{X}$

$\gamma$ nucleus $\rightarrow$ charm $X$

$\gamma$ nucleus $\rightarrow$ charm $\overline{\text { charm }} \mathrm{X}$

$\gamma$ nucleus $\rightarrow \mu^{+} \mu^{-} \mathrm{X}$

$\gamma$ nucleus $\rightarrow$ lepton ${ }^{+}$lepton $-\mathrm{X}$
$<350 \mathrm{GeV} / \mathrm{c}$

Particles studied charmed-meson, charmed-baryon

Brief description Continues studies of FNAL-087 and -401 . Uses bremsstrahlung photons from a wide band $350 \mathrm{GeV}$ $( \pm 15 \%)$ electron beam, a new large-aperture multiparticle spectrometer, a beryllium target, and a silicon microstrip decay-vertex detector. Studies the dynamics of heavy quark photoproduction, and decays of charm particles. Data analysis in progress (July 96)

Journal papers IEEE TNS 30 (1983) 3768, NIM A225 (1984)

619, NIM A241 (1985) 107, NIM A25I (1986) 40, NIM A252 (1986) 366, PL B251 (1990) 639, PL B263 (1991) 584, NIM A 305 (1991) 48, NP (PROC SUPPL) 27 (1992) 207, PL B300 (1993) 190, PL B307 (1993) 262, PL B308 (1993) 193, PL B313 (1993) 253, PL B314 (1993) 477, PL B315 (1993) 203, PL B316 (1993) 197, PRL 70 (1993) 1381, PRL 70 (1993) 1755, PRL 70 (1993) 2058, PRL 71 (1993) 827, PL B321 (1994) 295, PL B323 (1994) 459, PL B328 (1994) 187, PL B328 (1994) 193, PL B331 (1994) 217, PL B338 (1994) 106, PL B340 (1994) 254, PRL 72 (1994) 324, PRL 72 (1994) 961, NC 107A (1994) 2025, PR D50 (1994) 2953, PL B346 (1995) 199, PL B35I (1995) 591, PL B354 (1995) 486, PL B357 (1995) 678, PL B359 (1995) 403, PL B363 (1995) $259, \mathrm{PL} \mathrm{C} 364$ (1995) 127, PL B365 (1996) 461, and PL B370 (1996) 222

Related expcriments FNAL-831

E-mail contact jcumalat@fnal.gov, jcumalat@fotolb.colorado.edu, butler@fnal.gov WWW Home-page http://www.hep.uiluc.edu/e687/

\section{FNAL-690}

Proposed Feb 1981, Approved Jul 1981, Nov 1983, Apr 1987 Began data-taking 1990, Completed data-taking Jan 1992) STUDY OF HADRONIC PRODUCTION AND SPECTROSCOPY OF STRANGE, CHARM AND BOTTOM PARTICLES AT THE TEVATRON

COLUMBIA U - A G Gara, B C Knapp ( $\checkmark$ Spokesperson) ILLINOIS U, URBANA - E Gottschalk

FERMILAB - D C Christian, G Gutierrez, A A Wehmann GUANAJUATO U - J Felix, G Moreno, M Reyes, M Sosa

MASSACHUSETTS U - M C Berriso, E P Hartouni,

M N Kreisler, S Lee, K Markianos, M Wang, D Wesson

Accelerator FNAL-TEV Detector Spectrometer

Reactions

$$
p p \rightarrow p \mathrm{X} \quad 800 \mathrm{GeV} / c
$$

Particles studied strange, charm, bottom

Brief description Initial goals include (1) a systematic study of exclusive reactions, particularly diffraction dissociation, (2) cataloging of the remaining stable charmed particles, with details of production and decay, and (3) determining the scale of bottom production. Uses an innovative spectrometer with a hardware processor. Current focus is on the study of the charm production cross-section in diffraction dissociation and spin/parity analysis of centrally produced light mesons. Data analysis in progress (July 96).

Journal papers NC $107 \mathrm{~A}$ (1994) 1847.

Related experiments BNL-766

E-mail contact $\times 825 b c k @$ nevis.nevis.columbia.edu

FNAL-740 (DZERO)

(Proposed Sep 1983, Approved Feb 1984, Began data-taking May 1992, In progress)

STUDY OF $p p$ COLLISIONS USING A LARGE DETECTOR AT DO

DO COLLABORATION

ANDES U, BOGOTA - B Gomez, R Gomez, B Hoencisen,

D Mendoza, P Mooney, P Nechev, J P Negret, J M R Roldan, A Serna, $M \notin$ Zanabria

ARIZONA U - K P Davis, D K Fein, G E Forden, J A Guida, R Jayanti, K A Johns, A M Narayanan, J P Rutherfoord, M A Shupe, D Vititoe

BOSTON U - I M Butler

BROOKHAVEN - B Gibbard, H A Gordon, N Graf, S A Kahn. J R Kotcher, S D Protopopescu, S Snyder

BROWN U - J W Bantly, D Cullen-Vidal, D Cutts, T Fahland,

J M Guida, J S Hoftun, F Nang, $R$ A Partridge, G T Watts BUENOS AIRES U -- S Grinstein, R Piegaia

UC, DAVIS - P C Bloom, R Breedon, Y V Fisyak, S M Glenn,

G Grim, C B Klopfenstein, W Ko, R L Lander, S Mani,

D E Pellett

UC, IRVINE - J Drinkard, G Griffin, R E Hail, A J Lankford, D Stoker, J N Tarazi

UC, RIVERSIDE - J H Cochran, Jr , I A Ellison, P Gartung,

$\Lambda$ P Heinson, $M$ Mason, S J Wimpenny

RIO DE JANEIRO, CBPF - G A Alves, W P Carvalho,

$\mathrm{H}$ Da Motta Filho, $J \mathrm{M}$ De Miranda, A $\mathrm{K}$ A Maciel, M Mendes,

J M Miranda, L J P Moreira, M Nicola, A F D S Santoro,

M H G Souza, A Sznajder, M Vaz

MEXICO, IPN - H Castilla-Valdez, J L Gonzalez-Solis,

$R$ Hernandez-Montoya, $L$ Magana-Mendoza, $\Lambda$ SanchezHernandez

COLUMBIA U - I M Adam, A Kotwal, P M Tuts, B Winer DELHI U - V Bhasin, M Bhattacharjee, R K Shivpuri, S K Soni DUBNA - G D Alexeev, V I Dodonov, A V Efremov,

$Y$ A Gornushkin, M A Ignatenko, N N Khovansky,

Z V Krumstein, L K Lytkin, V L Malyshev, A A Nozdrin,

I L Pisarev, T O Rudenko, N A Russakovich, B M Sabirov,

A B Sadovsky, Y V Sedykh, A N Sissakian, L G 'Thatchev,

V V Tokmenine, E G Tskhadadze, L S Vertogradov,

Y A Yatsunenko, A I Zinchenko

FERMILAB - S C Ahn, B Baldin, I F Bartlett, P C Bhat, G C Blazey, A S Boehnlein, F O Borcherding, A Brandt, A D Bross, J H Christenson, W E Cooper, M Demarteau, D S Denisov, II $\mathrm{T}$ Diehl, $M$ Diesburg, S Feher, H E Fisk. $S$ C Fuess, K Genser, C E Gerber, D R Green, H B Greenlee. W X Gu, H F Haggerty, S Hansen, U Heint $z$, J D Hobbs, $\Upsilon \mathrm{Hu}$, A S Ito, M E Johnson, A M Jonckheere, $H$ Jostlein, B Klima, S Krzywdzinski, G Landsberg, Q H Li-Dcmarteau, R J Eipton,

Q Liu, L Lueking, H S Mao, M I Martin, H L Melanson. $\mathrm{K} W$ Merritt, C S Mishra, N Mokhov, H E Montgomery ( $\checkmark$ Spokesperson), M Narain, $N$ Oshima, A Para, PZ Quintas, 


\section{SUMMARIES OF FERMILAB EXPERIMENTS}

R Raja, P A Rapidis, W Smart, R P Smith, M A Tartaglia, W J Womersley, R Yamada

FLORIDA U - G Mitselmakher

FLORIDA STATE U - S K Blessing, S L Hagopian, V Hagopian,

T C Hicuring, $\mathrm{R} J$ Hirosky, S L Linn, R W Madden

J McDonald, H Piekarz, H B Prosper, C Shaffer, H D Waht,

G L Wang, S Youssef

HAWAII U - J Balderston, M A Cummings, M D Jones, M W Peters, C Y Yoshikawa

ILLINOIS U, CHICAGO - M R Adams, M Chung, H S Goldberg,

T M McKibben, II , C R Murphy, J Solomon

INDIANA U - G Alvarez, T Hu, R L Jesik, T R Marshall, D Zieminska, A Zieminski

IOWA STATE U - E W Anderson, J M Hauptman, B Lauer,

J A Wightman

KOREA U - J S Kang, C L Kim

KYUNGSUNG U .. Y M Park

CRACOW - A Eskreys, J Figicl, B Pawlik, P Stopa

LBL - A R Clark, O I Dahl, P M Grudberg, S C Loken,

R J Madaras, M L Stevenson, MW Strovink, T G Trippe, E W Varnes

MARYLAND U - A R Baden, W G Cobau, S C Eno, G Gomez, N J Hadley, S Kunori, A L Lyon, P Tamburello, J A Thompson MICHIGAN U - N A Amos, S Chopra, K W Del Signore, $T$ C F Hsich, D W Lincoln, H A Neal, L Oesch, J M Qian

MICHIGAN STATE U - M A Abolins, R L Brock, G Diloreto,

D L Edmunds, E M Flattum, K C Frame, T L Geld,

R J Genik, II , S A Jerger, F Landry, J T Linnemann,

J McKinley, D P Owen, B G Pope, T D Rockwell, N Varelas,

II J Weerts

MOSCOW STATE U - A Belyaev, E E Boos, L V Dudko,

P F Ermolov, A K Leflat, M Merkin, A Pukhov, E K Shabalina, E Sirotenko, N Sotnikova, E G Zverev

NEBRASKA U - J Krane, G R Snow

NEW YORK U - B K Abbott, A Mincer, M Mudan, P Nemethy, J Sculli, K R T Streets

NORTHEASTERN U - E Amidi, S M Chang, J H Morimisato,

S Reucroft, E von Gocler, D R Wood, T Yasuda

NORTHERN ILLINOIS $\mathrm{U}-\mathrm{M} R$ Fortner, J M Green, D R Hedin, $\mathrm{R}$ Markeloff, V I Sirotenko, S E Willis

NORTHWESTERN U - I Bertram, D A Buchholz, B Gobbi,

'I' Joffc-Minor, S Y Jun, B May, P Rubinov, H M Schellman, R M Snihur, T L Taylor

NOTRE DAME U - J Bishop, N N Biswas, J Jaques, R L Kehoe, M L Kelly, R C Ruchti, J Warchol, M Wayne

OKLAHOMA U - G M Guglielmo, P Gutierrez, G R Kalbfleisch, T M McMahon, J M Snow, M Strauss

PANJAB U - S Beri, V Bhatnagar, J M Kohli, H Singh, J Singh, $P$ M Sood

ST PETERSBURG, INP -- V Golovtsov, V Kim, P V Neustroev, N K Terentev, L Uvarov

SERPUKHOV - V V Babintsev, V A Bezzubov, N I Bojko,

V S Burtovoi, S V Chekulaev, S P Denisov, O V Eroshin,

V N Evdokimov, A N Galyaev, P I Goncharov, S N Gurzhiev,

Y E Gutnikov, B I Klochkov, V I Klyukhin, V I Kochetkov,

A V Kostritskii, A V Kozelov, E A Kozlovski, A A Mayorov,

V M Podstavkov, D A Stoianova, A A Volkov, A P Vorobiev

PURDUE U - S Carabello, D S Koltick, I Levine,

$Y$ M Pischalnikov

RICE U . D L Adams, G W Eppley, H E Miettinen, B P Padley, E Platner. P P Yepes

RIO DE JANEIRO STATE U - J R $\mathrm{T}$ de Mello Neto, J G R Lima, V Oguri

ROCHESTER U - D P Casey, C E Cretsinger, M K Fatyga,

T Ferbel, S Grunendahl, KS Hahn, F Lobkowicz, M F Paterno, E Won, Z H Zhu, M Zielinski

SACLAY - Y Ducros, 3 F Lebrat, A Zylberstejn

SEOUL NATIONAL U - S Choi, S K Kim, Y S Yu

SUNY, STONY BROOK - M M Baarmand, Z Casilum,

D Chakraborty. W M Chen, S Cinar, D R Claes, V D Elvira,

R J Engelmann, G Finocchiaro, A Goussiou, P D Grannis

( $\checkmark$ Spokesperson), T Hu, C K Jung, H L Li, R L McCarthy,

S Rajagopalan, M M Rijssenbeek, R D Schamberger, D Shpakov, Z Z Zhang

'ATA INS'l'.- B S Acharya, S Banerjee, S R Dugad, A Gupta,

M R Krishnaswamy, N K Mondal, V S Narasimham, N Parua,

M V S Rao, H C Shankar, P R Vishwanath

TEXAS U, ARLINGTON - K De, P A Draper, E J Gallas, J Li,

J Perkins, L Sawyer, S Shin, M D Sosebec, $R$ W Stephens,

A P White
TEXAS A AND M - L T Goss, F R Huson, D M Norman, 3 'T White, J V D Wirjawan

Accelerator FNAL-COLLIDER Detector DO

Reactions

$\bar{p} p \quad 2000 \mathrm{GeV}\left(\mathrm{E}_{\mathrm{cm}}\right)$

Particles studied $W^{+}, W^{-}, Z^{0}$, bottom, top

Brief description The experiment studies the properties of

2-TeV $\bar{p} p$ collisions with particular emphasis on measurement and identification of jets, leptons and missing transverse momentum. The detector incorporates three main systcms: a central detector, uranium-liquid argon calorimetry over nearly $4 \pi$ solid angle, and a magnetized iron muon spectrometcr. Data was taken from 1992 to early 1996 (Run I), with physics covering the top quark properties, precision electroweak measurements, studies of $\mathrm{QCD}, b$-quark production and searches for new particles and phenomena. The detector is being upgraded for operation in 1999 with the higher luminosity upgraded Collider. Tracking detectors will be replaced with silicon strip and scintillating fiber detectors in a solenoidal magnetic field, and other detectors upgraded for higher rate operation. Upgrade in progress (July 96).

Journal papers IEEE TNS 32 (1985) 1473, NIM A244 (1986) 356, NIM $\Lambda 247$ (1986) 107, CPC 45 (1987) 245, IEEE TNS 34 (1987) 710, NIM A256 (1987) 305, NIM A257 (1987) 556, NIM A261 (1987) 420, NIM A263 (1988) 78, NIM A265 (1988) 157, NIM A269 (1988) 492 [erratum: NIM A273 (1988) 453], NIM A277 (1989) 401, NIM A279 (1989) 107, NIM A279 (1989) 243, NIM A279 (1989) 310, NIM A279 (1989) 331, NIM A279 (1989) 359, NIM A280 (1989) 36, IEEE TNS 36 (1989) 384 NIM A289 (1990) 438, NIM A289 (1990) 543, NIM A290 (1990) 122 , NIM A290 (1990) 346, NIM A293 (1990) 125, NIM A297 (1990) 121, IEEE TNS 38 (1991) 286, IEEE TNS 38 (1991) 398, NP (PROC SUPPL) 23B (1991) 402, NIM A324 (1993) 53 , NIM A325 (1993) 393, NP (PROC SUPPL) 32 (1993) 29 NP (PROC SUPPL) 32 (1993) 83, NIM A338 (1994) 185, NIM A342 (1994) 33, NIM A351 (1994) 72, NIM A351 (1994) 77,

PRL 72 (1994) 965, PRL 72 (1994) 2138, PRL 72 (1994) 2332, NIM A366 (1995) 263, NP (PROC SUPPL) 44 (1995) 12, NP (PROC SUPPL) 44. (1995) 153, PL B357 (1995) 500, PL B358 (1995) 405, PRL 74 (1995) 2422, PRL 74 (1995) 2632, PRL 74 (1995) 3548, PRL 75 (1995) 6I8, PRL 75 (1995) 1023, PRL 75 (1995) 1028, PRL 75 (1995) 1034, PRL 75 (1995) 1456, PRL 75 (1995) 3226, PRL 75 (1995) 3618, PR D52 (1995) 4877, PL B370

(1996) 239, PRL 76 (1996) 734, PRL 76 (1996) 2222, PRL 76

(1996) 2228, PRL 76 (1996) 3271, and PR D53 (1996) 6000.

Related experiments FNAL-823

E-mail contact mont@fnal.gov,

grannis@sbhep.physics.sunysb.edu

WWW Home-page http://dosgio.fnal.gov/

FNAL-741 (CDF)

(Proposed Aug 1981, Approved Apr 1982, Began data-taking 1987, In progress

STUDX OF $\bar{p} p$ COLLISIONS USING A LARGE DETECTOR AT BO - THE CDF DETECTOR

CDF COLLABORATION

ARGONNE -- R E Blair, K Byrum, D Crane, S Kuhlmann, 'I LeCompte, L J Nodulman, J Proudfoot, R G Wagner, A B Wicklund

BOLOGNA U \& INFN, BOLOGNA - V Bolognesi, L Breccia, M Deninno, G Farinelli, I Fiori, L Malferrari, P Mazzanti, N Moggi, G M Piacentino, F Rimondi, F Semeria, S Zucchelli

BRANDEIS $U$ - S Behrends, J R Bensinger, C Blocker,

L E Kirsch, J Lamoureux

CHICAGO U - A Amadon, J Berryhill, M Contreras,

R Culbertson, H J Frisch, C Grosso-Pilcher, M J Shochet,

D A Toback, J Wahl, P Wilson

DUKE U - D Cronin-Mennessy, J R Dittmann, L Fortney, A $T$ Goshaw, $S$ A Hauger, W Kowald, S H Oh, $T$ Phillips, W J Robertson, W Walker, C H Wang, C Wei

FERMILAB - M G Albrow, M Atac, A Beretvas, J P Berge, K Bicry, M E Binkley, E Buckley-Geer, A Byon-Wagner, 


\section{SUMMARIES OF FERMILAB EXPERIMENTS}

S Cihangir, J Cooper, S Delchamps, $\mathrm{R}$ Demina, $\mathrm{P}$ Derwent, $\mathrm{F}$ DeJongh, J E Elias, W Erdmann, B Flaugher, G W Foster, J E Freeman, S Geer, S R Hahn, R Harris, R Hughes, J Hylen, J Incandela, H B Jensen, U Joshi, D Jovanovic, R D Kephart, W Koska, I I Kroll, S Lammel, J D Lewis, P Limon, P Lukens, K Maeshima, J P Marriner, T Miao, A Mukherjee, C A Nelson, C Newman-Holmes, J F Patrick, K Pitts, R Plunkett,

P Schlabach, E E Schmidt, S L Segler, J Spalding, L Spiegel, J Strait, D Stuart, S Tkaczyk, A V Tollestrup, R Vidal, R L Wagner, W Wester, E Wicklund, A Yagil, G P Yeh, J Yoh, $\mathrm{J}$ C Yun

FRASCATI - M Barone, E Barzi, S Bertolucci, M Cordelli, $S$ Deli'Agnello, F Donno, P Giromini, L Keeble, E Meschi, S Miscetti, A Parri, A Sansoni

GENEVA U - A Clark, C Couyoumtzelis, II Kambara, T Speer, $\mathrm{X}$ Wu

HARVARD U - T P Baumann, J F de Troconiz, M Franklin, C Gay, A Gordon, R Hamilton, J E Futh, D Kestenbaum, J Konigsberg, G Michail, O Poujade, F Ptohos, M G Spiropulu HIROSHIMA U \& OSAKA U - Y Iwata, T Ohmoto, T Ohsugi, R Takashima, N Tamura

ILLINOIS U, URBANA - L Christafek, D Errede, S M Errede, L E Holloway, R M Keup, B Kharadia, T Liss, A J Martin, R M Roser

IPP, CANADA \& MCGILL U \& TORONTO U - B Hinrichsen, A Holscher, H S Kim, $K$ Kordas, K Ragan, G Sganos, P Sinervo, K Strahl, W J Taylor, W Trischuk, A Warburton, Y Ye JOINS HOPKINS U - B A Barnett, J Cammerata, $Z$ Feng, D Gerdes, J Guimaraes, J Skarha, C Smith, F Snider, J Tseng KEK - Y Fukui, S Mikamo, Y Morita

LBL - W Ashmanskas, W Carithers ( $\sqrt{ }$ Spokesperson),

W Chinowsky, K Einsweiler, R P Ely, A B Galtieri, M GarciaSciveres, C H Haber, R Kadel, Y K Kim, M Lancaster, J Lys, M Paulini, M D Peters, D Reher, M Shapiro, J L Siegrist, H Wenzel, W Yao

MIT, LNS - G Bauer, J Benlloch, W Bokhari, T Daniels, J Friedman, E Hafen, K Kelley, A Korytov, P Maksimovic, C-Y P Ngan, L Rosenson, T Shah, P Sphicas, S Sumorok, $S$ Tether, $D$ Vucinic

MICHIGAN STATE U - C Bromberg, J Huston, R Miller, S Murgia

MICHIGAN U - D Amidei, K Burkett, M Campbell, J Chapman, N Eddy, E Guilian, E James, S-B Kim, M Krasberg, C-C Miao, $R$ Thun, S Truitt, S Vejcik, D Winn, D Wolinski

NEW MEXICO U - . M Bailey, N Bruner, M Gold, J Matthews, E Moore, S Seidel, T $L$ Thomas, S Worm, L Yu

OSAKA CITY U - Y Kato, T Okusawa, T Takahashi, $\mathrm{X}$ Teramoto, H Toyoda, $\mathrm{T}$ Yoshida

PADUA U \& INFN, PADUA - P Azzi, N Bacchetta, D Bisello, G Busetto, A Castro, T Dorigo, M Gallinaro, Y Gotra, $M$ Loreti, F Mando, L Pescara, A Ribon, L Stanco, J Wyss

PENN U - F Azfar, D Benton, B Bevensee, L Gladncy, B Harral, J Heinrich, C Holck, R J Hollebeek, G Houk, N Lockyer, $O$ Long, S D Metzler, R Oliveira, F Ukegawa, G Unal, R Wilkinson, H H Williams

INFN, PISA \& PISA, SCUOLA NORMALE SUPERIORE \& PISA U - S R Amendolia, F Bedeschi, S Belforte, G Bellettini ( $\checkmark$ Spokesperson), S Bettelli, F Cervelli, G Chiarelli, M Cobal, E Cocca, M Dell'Orso, B Denby, S Donati, C Ferretti, G Gagliardi, S Galeotti, P Giannetti, M Incagli, N Labanca, $\mathrm{S}$ Lami, $\mathrm{S}$ Leone, $\mathrm{M} \mathrm{L}$ Mangano, A Menzione, $\mathrm{P}$ Murat, R Paoletti, N Parashar, A Perazzo, G Punzi, L F Ristori, A Scribano, F Spinella, A Stefanini, G F Tartarelii, N 'Turini, $G$ Velev, F Zetti

PITTSBURGH U - J Boudreau, E Engels, Jr, T Huffman, P F Shepard, P P Singh, $S$ van den Brink

PURDUE U - V E Barnes, D Bortoletto, M Fahling, A F Garfinkel, $A$ Hardman, K Hoffman, T Keaffaber, A $\Upsilon$ Laasanen, $N$ M Shaw, $Q$ Shen

ROCHESTER U - S Blusk, $\Lambda$ Bodek, H S Budd, J Cassada, $\mathrm{P}$ de Barbaro, Q Fan, B Kim, P Koehn, M Kruse, J Liu, M Pillai, W K Sakumoto, $P$ Tipton, $K$ Tollefson

ROCKEFELLER U - A Akopian, G Apollinari, S Bagdasarov, A Bhatti, L Demortier, N D Giokaris, K Goulianos, D Khazins, A Maghakian, P Melese, C Mesropian, A Titov

RUTGERS U - J Conway, T J Devlin, L Groer, C Hawk, V Jacobs, R D Kennedy, E W Kuns, C Loomis, Jr, M Walsh, T L Watts
TAIWAN, INST PHYS - P Chang, P Chang, $\mathrm{H}$ Y Chao, M$T$ Cheng, C-N Chiou, R Guo, Y-C Liu, A Soumarokov, P K Teng, C-H Wang, M-J Wang, P Yeh

TEXAS A AND M - J Done, T Kamon, P M McIntyre, B Tannenbaum, R C Webb

TEXAS TECH - D Benjamin, M Frautschi, O Ganel, W Hao, $Q$ Liu, V Papadimitriou, A Sill, $\mathrm{R}$ Wigmans

TSUKUBA U - S Aota, T Asakawa, 'T Chikamatsu, S' Funaki, K Hara, E Hayashi, H Ikeda, T Ino, T Kaneko, S Kim,

K Kondo, T Kuwabara, H Minato, H Mitsushio, S Miyashita, H Nakada, I Nakano, M Ninomiya, S Ogawa, R Oishi, M Okabe, H Sato, Y Sciya, M Shimojima, J- Suzuki, T Takada, M Takano, T Takano, K Takikawa, T Uchida, N Uemura, K Yasuoka, $M$ Yokoyama

TUFTS U - K Karr, K Sliwa, M 'rimko

UCLA - Y Bonushkin, H Dahnke, L Dworkin, J Hauser,

F Keyvan, M Lindgren, T Muller, D Neuberger

WASEDA U - H Akimoto, 'T Arisawa, $Y$ Fujimoto, S Hasegawa, $J$ Iwai, $K$ Terashi, $S$ Uesaka, $Y$ Yoda

WISCONSIN U - J Bellinger, D L Carlsmith, W Chung,

R M Handler, S Lusin, J Olsen, L G Pondrom, J Steele, L Zhang

YALE U - R G Feild, H Kasha, K E Ohl, S Pappas, A Schaffer, M P Schmidt

Accelerator FNAL-COLLIDER Detector CDF

Reactions

$\bar{p} p \quad 500-2000 \mathrm{GeV}\left(\mathrm{E}_{\mathrm{cm}}\right)$

Particles studied $W^{+}, W^{-}, Z^{0}$, higgs, top

Brief description The first physics results were obtained during 1987 , in an engineering $\mathrm{run}$, and in $1988 / 89$, in a year-long run. Upgrades for the 1991 run are described in the FNAL775 proposal, and another major improvement was proposed for the 1993 run. CDF is a general-purpose detector designed to study the physics of $p \bar{p}$ collisions. It has both azimuthal and forward-backward symmetry. A superconducting solenoid of length $4.8 \mathrm{~m}$ and radius $1.5 \mathrm{~m}$ generates a $1.4 \mathrm{~T}$ magnetic field and contains tracking chambers used to detect charged particles and measure their momenta. Surrounding the solenoid are sampling calorimeters used to measure the electromagnetic and hadronic energy of jets and electrons. Outside the calorimeters are drift chambers used for muon detection. Surrounding the beam pipe is a 4 -layer silicon microstrip vertex detector, and a vertex drift chamber, both installed in 1992. The Collider run ended in Spring 96 with a total luminosity of $110 \mathrm{pb}^{-1}$ recorded on tape. The detector will be upgraded with new tracking, vertex detector, scintillating tile plug calorimeter, readout and trigger electronics, and extended muon coverage for the next Collider run with the Main Injector, now scheduled for April, 1999. Upgrade in progress (July 96).

Journal papers NIM 204 (1983) 351, NIM 204 (1983) 361, NIM 205 (1983) 113, NIM 216 (1983) 127, NIM A219 (1984) 472, JdeP 45 (1984) 333, NIM A238 (1985) 18, IEEE TNS 34 (1987) 865, NIM A263 (1988) 199, NIM A267 (1988) 249, NIM A267 (1988) 257, NIM A267 (1988) 272, NIM A267 (1988) 280, NIM A267 (1988) 301, NIM A267 (1988) 315, NIM A267 (1988) 330, NIM A267 (1988) 351, NIM A268 (1988) 24, NIM A268 (1988) 33, NIM A268 (1988) 41, NIM A268 (1988) 46, NIM A268 (1988) 50, NIM A268 (1988) 75, NIM A268 (1988) 92, NIM A269 (1988) 33, NIM A269 (1988) 40, NIM A269 (1988) 51, NIM A269 (1988) 63, NIM A269 (1988) 68, NIM A269 (1988) 82, NIM A269 (1988) 93, NIM A271 (1988) 387, PRL 61 (1988) 1819, PRL 62 (1989) 613, PRL 62 (1989) 1005, PRL 62 (1989) 1825, PRL 62 (1989) 3020, PRL 63 (1989) 720, PRL 63 (1989) 1447, NIM A274 (1989) 443, NIM A281 (1989) 485, PR D40 (1989) 3791, NP A498 (1989) 193c, IEEE TNS 36 (1989) 35 IEEE TNS 36 (1989) 347 , IEEE TNS 36 (1989) 440 , IEEE TNS 36 (1989) 765, NP (PROC SUPPL) 12 (1990) 18, NP (PROC SUPPL) 12 (1990) 254, PRL 64 (1990) 142, PRL 64 (1990) 147, PRL 64 (1990) 152, PRL 64 (1990) 157, PRL 64 (1990) 348. PRL 65 (1990) 968, PRL 65 (1990) 2243, PR D4l (1990) 1717, PR D4I (1990) 1722, PR D41 (1990) 2330, PRL 66 (1991) 2951, PRL 67 (1991) 1502, PRL 67 (1991) 2418, PRL 67 (1991) 2609, PRL 67 (1991) 2937, PRL 67 (1991) 3351, PR D43 (1991) 664, PR D43 (1991) 2070, PR D44 (1991) 29, PR D44 (1991) 601, NIM A315 (1992) 125, NP (PROC SUPPL) 25B (1992) 19, NP (PROC SUPPL) 27 (1992) 240, NP (PROC SUPPL) 27 (1992) 246, MPL A7 (1992) 2659, PRL 68 (1992) 447, PRL 68 (1992) 1458, PRL 68 (1992) 1463, PRL 68 (1992) 2734. PRL 68 


\section{SUMMARIES OF FERMILAB EXPERIMENTS}

(1992) 3398, PRL 68 (1992) 3403, PRL 69 (1992) 28, PRL 69 (1992) 2160, PRL 69 (1992) 2896, PRL 69 (1992) 3439, PRL 69 (1992) 3704, PR D45 (1992) 1448, PR D45 (1992) 2249, PR D45 (1992) 3921, PR D46 (1992) 1889, NIM A331 (1993) 57, NIM A 333 (1993) 209, NP (PROC SUPPL) 31 (1993) 189, PRL 70 (1993) 713 , PRL 70 (1993) 1376, PRL 70 (1993) 2232, PRL 70 (1993) 4042, PRL 71 (1993) 500, PRL 71 (1993) 679, PRL 71 (1993) 1685, PRL 71 (1993) 2396, PRL 71 (1993) 2537, PRL 71 (1993) 2542, PRL 71 (1993) 3421, PR D47 (1993) 2639, PR D47 (1993) 4857, PR D48 (1993) 998, PR D48 (1993) 2998, PR D48 (1993) 3939, NIM A350 (1994) 73, NIM A351 (1994) 59, NIM A351 (1994) 68, PRL 72 (1994) 1977, PRL 72 (1994) 3004, PRL 72 (1994) 3456, PRL 73 (1994) 220, PRL 73 (1994) 225, PRL 73 (1994) 2296, PRL 73 (1994) 2662 [erratum: PRL 74 (1995) 1891], PRL 73 (1994) 2667, NC 107A (1994) 2085, PR D49 (1994) 1, PR D50 (1994) 2966, PR D50 (1994) 4252, PR D50 (1994) 5518, PR D50 (1994) 5535, PR D50 (1994) 5550, PR D50 (1994) 5562, NP (PROC SUPPL) 39BC (1995) 348, NP (PROC SUPPL) 44 (1995) 20, NIM A368 (1995) 90, PRL 74 (1995) 341 PRL 74 (1995) 850, PRL 74 (1995) 855, PRL 74 (1995) 1936 PRL 74 (1995) 1941, PRL 74 (1995) 2626, PRL 74 (1995) 2900, PRL 74 (1995) 3538, PRL 74 (1995) 4988, PRL 75 (1995) 11, PRL 75 (1995) 608, PRL 75 (1995) 613, PRL 75 (1995) 1012, PRL 75 (1995) 1017, PRL 75 (1995) 1451, PRL 75 (1995) 3068, PRL 75 (1995) 3997, PRL 75 (1995) 4358, PR D51 (1995) 949, PR D51 (1995) 4623, PR D52 (1995) 2605, PR D52 (1995) 2624, PR D52 (1995) 4784, IJMP Al1 (1996) 2045, IJMP Al1 (1996) 2233, PRL 76 (1996) 2006, PRL 76 (1996) 2015, PRL 76 (1996) 2852, PRL 76 (1996) 3070, PRL 76 (1996) 4307, PRL 76 (1996) 4462, PRL 76 (1996) 4675, PR D53 (1996) 1051, and PR D53 (1996) 3496

Related experiments FNAL-775, FNAL-830, FNAL-876

E-mail contact bellettini@pisa.infn.it, bellettini@fnal.gov, carithers@fnal.gov

WWW Home-page http://www-cdf.fnal.gov/

\section{FNAL-760}

(Proposed Mar 1985, Approved Jun 1985, Began data-taking 1990, Completed data-taking Jan 1992)

INVESTIGATION OF THE FORMATION OF CHARMONIUM STATES USING THE $\bar{p}$ ACCUMULATOR RING

UC, IRVINE ‥ D R Broemmelsiek, J E Fast, K E Gollwitzer, M A Mandelkern, J L Marques, J Schultz, A Smith, M F Weber, $G$ Zionlas

FERMILAB - L Bartoszek, V K Bharadwaj, M D Church,

A A Hahn, S Y IIsueh, W L Marsh, J Peoples, Jr, S H Pordes, $P$ A Rapidis, S Werkema

FERRARA U - D Bettoni, R Calabrese, V Carassiti, P Dalpiaz, M Fabbri, P Ferretti-Dalpiaz, A Gianoli, E Luppi, M Martini, F Petrucci, M Savrie

INFN, GENOA - A Buzzo, M Macri, M M Marinelli,

M Pallavicini, S Passaggio, C Patrignani, M G Pia, A Pozzo. A Santroni, A Scalisi, M Zito

NORTHWESTERN U - D A Dimitroyannis, C M Ginsburg, M Masuzawa, R E Ray, Jr, J L Rosen, M Sarmiento, K K Seth, $S$ Trokenheim. J $\mathrm{L} J$ Zhao

PENN STATE U - T A Armstrong, M A Hasan, R A Lewis, A M Majewska, J Passaneau, J D Reid, G A Smith, Y Zhang 'IURIN U - C Biino, G Borreani, A Ceccucci, R Cester ( $\checkmark$ Spokesperson), R Dibencdetto, G Giraudo, F Marchetto, E A Menichetti, A Migliori, R Mussa, S Palestini, N M Pastrone, L Pesando, G Rinaudo, B Roccuzzo, M S Sozzi, L Tecchio Accelerator FNAL Detector Tracking system, Calorimeter Reactions

$$
\begin{array}{lc}
\bar{p} p \rightarrow \text { charm } \overline{\text { charm }} & 3-7 \mathrm{GeV} / c \\
\bar{p} p \rightarrow e^{+} e^{-} & " \\
\bar{p} p \rightarrow \gamma^{\prime} \mathrm{s} & " \\
\bar{p} p \rightarrow \bar{p} p & "
\end{array}
$$

Particles studied charmonium

Brief description Studies charmonium states formed exclusively in $p p$ collisions, and their decays to electromagnetic final states. Uses a gas jet hydrogen target in the Fermilab $\vec{p}$ source. The detector consists of a tracking system, hodoscopes. and Čerenkov counters surrounded by a central lead glass electromagnetic calorimeter, and a planar forward calorimeter. Data analysis completed.

Journal papers NIM A271 (1988) 417, NIM A277 (1989) 116 , NIM A295 (1990) 73, NIM A301 (1991) 47, NIM A307 (1991) 254, NIM A317 (1992) 135, SJNP 55 (1992) 792, SJNP 55 (1992) 811, SJNP 55 (1992) 865, PRL 68 (1992) I468, PRL 69 (1992) 2337, NP B373 (1992) 35, PL B307 (1993) 394, PL B307 (1993) 399, PRL 70 (1993) 1212, PRL 70 (1993) 2983, NP A558 (1993) 259c, PR D47 (1993) 772, PR D48 (1993) 3037, NC 107A (1994) 2013, PAN 57 (1994) $1513=$ YF 57 (1994) 1587, PAN 57 (1994) $1722=$ YF 57 (1994) 1793, NIM A355 (1995) 308, and PR D52 (1995) 4839.

Related experiments FNAL-835

E-mail contact cester@to.infn.it, cester@fnal.gov WWW Home-page http://www-e835.fnal.gov/

\section{FNAL-771}

(Proposed Feb 1986, Approved Apr 1987, Began data-taking 1991 Completed data-taking Jan 1992)

\section{BEAUTY PRODUCTION AND OTHER HEAVY QUARK PHYSICS ASSOCIATED WITH DIMUON PRO- DUCTION IN $800(925) \mathrm{GeV} / c \quad p$ Si INTERACTIONS}

SOUTH ALABAMA U - R K Clark, C M Jenkins

UC, BERKELEY - H C Ballagh, Ir , H H Bingham, I E Lys, $S$ Misawa

UCLA - A F Boden, D B Cline, S Ramachandran, J M Rhoades DUKE U - L R Fortney, W R Kowald, C Wei, B T Zou

FERMILAB - P O Mazur, C T Murphy, R P Smith, L Spiegel,

W Yang

HOUSTON U - K H Lau, G H Mo

DUBNA - J Budagov

LECCE U - P Creti, V Elia, E Gorini, F Grancagnolo, M Panareo

MCGILL U - J M 'Trischuk

NANJING U - T Y Chen, N G Yao

NORTHWESTERN U - M M Block

PAVIA U - L Antoniazzi, G Bonomi, G Introzzi, G Liguori, P Torre PENN U - A J Blankman, W I Kononenko, W Selove

PRAIRIE VIEW A AND M - M L IIaire, D I Judd, L Turnbull,

D E Wagoner

SHINSHU U - M He, $\mathrm{C} H$ Shen, $C$ Wang, $C$ Wei, $N$ Zhang

VANIER COLL - M S Cooper

VIRGINIA U - M W Arenton, Z L Cao, S Conetti

( $\sqrt{ }$ Spokesperson), G Corti, B B Cox ( $\sqrt{ }$ Spokesperson),

E C Dukes, C M Durandet, V Golovatyuk, K Hagan-Ingram,

$P$ M Hanlet, A A Ledovskoy, A P Mcmanus, K S Nelson,

V S Pogosyan, M Recagni, J Segal, I Tramouranis

WISCONSIN U - T Alexopoulos, A R Erwin, J R Jennings

Accelerator FNAL-TEV Detector Spectrometer

$\begin{array}{cc}\frac{\text { Reactions }}{p \mathrm{Si}} \rightarrow \mu^{+} \mu^{-} \mathrm{X} & 800 \mathrm{GeV} / c \\ p \mathrm{Si} \rightarrow \operatorname{muon} \mathrm{X} & " \\ p \mathrm{Si} \rightarrow B \bar{B} \mathrm{X} & " \\ p \mathrm{Si} \rightarrow J / \psi(1 S) \mathrm{X} & " \\ p \mathrm{Si} \rightarrow \chi_{c} \text { (unspec) } \mathrm{X} & "\end{array}$

Particles studied $B^{+}, B^{0}, J / \psi(1 S), \psi(2 S), \chi_{c 1}(1 P), \chi_{c}$ (unspec)

Brief description Uses the PNAL-705 spectrometer augmented

by a 10,000-channel silicon detector and a new single muon and dimuon trigger to select $B \bar{B}$ events at a high rate $\left(\sim 2 \times 10^{6} / \mathrm{s}\right)$. Data analysis in progress (July 96).

Journal papers NP (PROC SUPPL) $23 \mathrm{~B}$ (1991) 249, NIM A314 (1992) 563, NIM A315 (1992) 92, NIM A333 (1993) 142, NIM A337 (1993) 350, NIM A340 (1994) 491, NIM A355 (1995) 320 , NIM A360 (1995) 334, PL B374 (1996) 271, and PRL 77 (1996) (in press).

E-mail contact cox@uvahep.phys.virginia.edu, cox@fnal.gov, sergio@virginia.edu, sergio@fnal.gov

WWW Home-page

http://fcrmi.clas.virginia.edu/ aal2q/e771/e771hp.html 


\section{SUMMARIES OF FERMILAB EXPERIMENTS}

\section{FNAL-773}

(Proposed Mar 1986, Approved Jul 1986, Jun 1989, Began datataking Jul 1991, Completed data-taking Sep 1991)

\section{MEASUREMENT OF THE PHASE DIFFERENCE} BETWEEN $\eta_{00}$ AND $\eta_{+-}$TO A PRECISION OF $0.5^{\circ}$

CHICAGO U - A R Barker, R A Briere, E Cheu, L K Gibbons, D Harris, G D Makoff, K S Mcfarland, A Roodman, $B$ Schwingenheuer, Y W Wah, B D Winstein, $R$ Winston ELMHURST COLL - E C Swallow

FERMILAB - G J Bock, R N Coleman, M Crisler, J Enagonio,

$R$ Ford, $X$ B Fisiung, D Jensen, E Ramberg, $R$ S Tschirhart,

$T$ Yamanaka

ILLINOIS U, URBANA - E Collins, G D Gollin ( $\checkmark$ Spokesperson)

RUTGERS U - P Gu, P Haas, W P Hogan, S K Kim,

J N Matthews, S S Myung, S R Schnetzer, S V Somalwar,

G B Thomson, Y Zou

Accelerator FNAL-TEV Detector Spectrometcr

Reactions

$\begin{array}{lc}K_{L} \rightarrow \pi^{+} \pi^{-} & 50-150 \mathrm{GeV} / c \\ K_{L} \rightarrow \pi^{+} \pi^{-} \gamma & " \\ K_{L} \rightarrow \pi^{0} \pi^{0} & " \\ K_{S} \rightarrow \pi^{+} \pi^{-} & " \\ K_{S} \rightarrow \pi^{+} \pi^{-} \gamma & " \\ K_{S} \rightarrow \pi^{0} \pi^{0} & "\end{array}$

Particles studied $K_{L}, K_{S}$

Brief description This experiment adds an additional regenerator to the FNAL-731 spectrometer. A double $K_{L}$ beam is incident on the spectrometer, which has 804 lead glass blocks and four drift chambers. One beam passes through a thin regenerator at the start of the fiducial decay volume, the other traverses a thick regenerator 11 meters further upstream. The regenerators switch beams between machine pulses. Neutral beam is produced by $800 \mathrm{GeV}$ protons on a $36-\mathrm{cm}$ beryllium target.

The experiment tests $C P T$ invariance. Data analysis completed (July 96).

Journal popers PRL 74 (1995) 4376, and PRL 75 (1995) 2803.

Related experiments FNAL-731, FNAL-799

E-mail contact gollin@postoffice.hep.uiuc.edu

FNAL-781

(Proposed Mar 1987, Approved Oct 1988, In preparation)

\section{SEGMENTED LARGE-X BARYON SPECTROMETER} (SELEX)

BEIJING, IHEP - $\mathrm{K} L \mathrm{He}$, F F Lang, C Z Li, Y S Li, Z G Li, C S Mao, Z L Mao, F K Tang, D R Wang, Y G Xi, J Q Zhang, W H Zhao, SC Zheng

BOGAZICI U - E Gulmez

BRISTOL U - V J Smith

CARNEGIE MELLON U - R M Edelstein, D Gibaut

E E Gottschalk, A Kushnirenko, D Mao, P Mathew, M Mattson,

D M Potter, M P Procario, I S Russ (Spokesperson), S Yang

RIO DE JANEIRO, CBPF - E C De Oliveira, A M F Endler, L C S Oliveira, M C Pommot Maia

FERMILAB - P S Cooper (Spokesperson), J Engelfried,

J R Kilmer, S Kwan, J Lach, G A Oleynik, E J Ramberg, D D Skow, L G Stutte

HAWAII U - C J Kenney, S I Parker

IOWA U - N Akchurin, M Aykac, K R Barger, M Kaya, U Mallik,

E R Mccliment, J M Mcpherson, K D Nelson, C R Newsom,

Y Onel, E Ozel, S Ozkorucuklu, L Pasquali

MUNICH, MAX PLANCK INST - U Dersch, I Eschrich,

K Koenigsmann, I Konorov, Hz Krueger, S Masciocchi, S M Panl,

B Povh, J Simon, K Vorwalter, R Werding

MOSCOW STATE U - Filimonov, E M Leikin, A Nemitkin,

$\checkmark$ Rud

MOSCOW, IlEP - M Balats, G Davidenko, A Dolgolenko,

G Dzyubenko, V Evdokimov, P A Goritchev, V M Guzhavin,

A Kamenski, V D Khovansky, M A Kubantsev, V S Lakaev,

$\mathrm{V}$ Matveyev, $A \mathrm{P}$ Nilov, $\mathrm{V} A$ Prutskoi, V K Semyachkin,

A L Sitnikov, V Verebryusov, V E Vishnyakov
PARAIBA U - M Euksys

ST PETERSBURG, INP - A Atamantchouk, N Bondar,

A S Denisov, A Goliach, V L Golovtsov, V T Gratchev,

A V Khanzadeev, V T Kim, L M Kotchenda, A G Krivshich,

N P Kuropatkin, V Malcev, P V Neustroev, V M Samsonov,

V A Schegelsky, N N Smirnov, V L Stepanov, M Svoiski,

N K Terentiev, L N Uvarov, A P Vorobiev

SERPUKHOV - $R$ Elochin, Y Goncharenko, O A Grachov,

V Koubarovski, A Kozhevnikov, N Kulyavtzev, V F Kurshetsov,

L G Landsberg, V Moltchanov, B A Mukhin, V Mukhin,

S B (S) Nurushev, A N Vasiliev, D V Vavilov, V A Victorov

ROCHESTER U - $T$ Ferbel, G E Ginther, $\mathrm{J} r$, C Fammer,

P F Slattery, M Zielinski

INFN, ROME - M Iori

SAN LUIS POTOSI U - A M Morelos-Pineda

SAO PAULO U - O P Eboli, L Emediato, C O Escobar, F Garcia, $P$ Gouffon, $T$ Lungov, $R$ Soares, $R$ Zukanovich-Funchal

TEL AVIV U i. S Gerson, J Grunhaus, S Kananov,

M A Moinester, A Ocherashvili, V Steiner

INFN, TRIESTE - A Bravar, D Dreossi, A Lamberto, $A$ Penzo. G F Rappazzo, P P Schiavon

WASHINGTON U, SEATTLE - V Chaloupka, 'T Zhao

Accelerator FNAL-TEV Detector Spectrometer

Particles studied charmed-baryon

Brief description Studies both charmed baryon production and decays. Trigger is based on impact parameter. The spectrometer deploys a number of existing detectors as well as the new silicon strip and pixel devices and a ring imaging Cerenkov counter. Being installed (May 96).

E-mail contact pcooper@fnal.gov, russ@cmphys.phys.cmu.edu WWW Home-page http://fn78la.fnal.gov/

\section{FNAL-789}

(Proposed Nov 1987, Approved Oct 1988, Began data-taking 1990. Completed data-taking Jan 1992)

MEASUREMENT OF THE PRODUCTION AND DECAY INTO TWO-BODY MODES OF b-QUARK MESONS AND BARYONS

ABILENE CHRISTIAN U - L D Isenhower, M E Sadler, $R$ G Schnathorst

TAIWAN, INST PHYS - Y C Chen, G C Kiang, P K Teng

CHICAGO U .. L M Lederman, M H Schub

FERMILAB … C N Brown, W E Cooper, H D Glass, K N Gounder, C S Mishra

LBL .. G Gidal, P M Ho, M S Kowitt, K B Luk, D Pripstein

LOS ALAMOS - T A Carey, D M Jansen, $R$ G Jeppesen, J S Kapustinsky, D W Lane, M J Leitch, J W Lillberg, P L McGaughey, J M Moss, J C Peng ( $V$ Spokesperson)

NORTHERN ILLINOIS U - D M Kaplan ( $V$ Spokesperson),

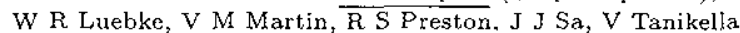
SOU'IH CAROLINA U - R L Childers, C W Darden. J R Wilson Accelerator FNAL-TEV Detector Spectrometer

Reactions

$p$ mucleus $\quad 800 \mathrm{GeV} / c\left(\mathrm{P}_{\mathrm{lab}}\right)$

Particles studied bottom, charm

Brief description Studies low multiplicity decays of $b$-and c-quark hadrons. Essential to evaluating the suitability of dihadronic beauty decays for the study of $C P$ violation in the $B$ system. Sensitive also to dileptonic modes, allowing limits to be set on their branching ratios. Uses the existing FNAL-605/772 spectrometer with suitably upgraded trigger processor system. Data analysis in progress (July 96).

Journal papers IEEE TNS 38 (1991) 461, IEEE TNS 39 (1992) 758, NP A544 (1992) 197c, PRL 72 (1994) 1318, PRL 72 (1994) 2542, PR D50 (1994) 9, PRL 74 (1995) 3118, PR D52 (1995) 1307, and PR D52 (1995) 4251.

Related experiments FNAL-771, DESY-HERA-B

E-mail contact peng@lanl.gov, kaplan@fnal.gov

WWW Home-page http://p2hp2.lanl.gov/e789/e789.html 


\section{SUMMARIES OF FERMILAB EXPERIMENTS}

\section{FNAL-791}

(Proposed Nov 1987, Approved Jun 1988, Began data-taking 1991, Completed data-taking Jan 1992)

\section{HADROPRODUCTION OF HEAVY FLAVORS AT THE} TAGGED PHOTON LABORATORY

E791 COLLABORATION

RIO DE JANEIRO, CBPF - S F Amato, I Bediaga,

I Costa, J M De Miranda, J C Dos Anjos, J Mello, Neto,

H Motta, Filho, A Reis, A Santoro, J Solano, M H G Souza

UC, SANTA CRUZ - G Blaylock, P Gagnon, J Leslie,

K O'Shaughnessy, K Sugano

CINCINNATI U - S Devmal, B Meadows, L P Perera,

A. K Santha, M D Sokoloff

FERMILAB - J A Appel ( $\checkmark$ Spokesperson), S Banerjee,

S B Bracker, T G Carter, L Chen-Tokarek, K Denisenko,

A $M$ Halling, C C James, S Kwan, B G Lundberg, K A Thorne

ILLINOIS TECH - $\mathrm{R}$ A Burnstein, P A Kasper, $\mathrm{K} C$ Peng,

II A Rubin

KANSAS STATE $U$ - M Aryal, A Nguyen, N W Reay,

R A Sidwell, N R Stanton, A Tripathi, N Witchey, S Yoshida,

C Zhang

MEXICO, IPN - A Gago, G Herrera-Corral

MISSISSIPPI U - E M Aitala, L M Cremaldi, K N Gounder,

A Rafatian, J J Reidy, D J Summers, D Y Yi

OHIO STATE U - K Reibel

PRINCETON U - D Langs, A Schwartz, J Wiener

PUEBLA U, MEXICO - E Cantoral, A B D'Oliveira, A Fernandez RIO DE JANEIRO U - H D S Carvalho, A I Ramalho

SOUTH CAROLINA U - N Copty, M V Purohit ( $\checkmark$ Spokesperson)

STANFORD U - P R Burchat, R Zaliznyak

TEL AVIV U - D Ashery, S Gerzon, G Iurvits, J Lichtenstadt,

$S$ MayTal-Beck, R Weiss-Babai

TUFTS U - R H Milburn, A Napier

WISCONSIN U - S A Radeztsky, M C Sheaff, K Stenson,

$S$ Watanabe

YALE U - C L Darling, A J Slaughter, S F Takach, E J Wolin

Accelerator FNAL-TEV Detector TPS

Reactions

$$
\pi^{-} \text {nucleus } \rightarrow \text { charm X } \quad 500 \mathrm{GeV}\left(\mathrm{E}_{\mathrm{lab}}\right)
$$

$\pi^{-}$nucleus $\rightarrow$ bottom $X$

Particles studied charm, bottom

Brief description Continues studies of FNAL-769. Emphasizes charm physics and a first look at bottom hadroproduction.

Targets are $\mathrm{Pt}$, and $\mathrm{C}$ foils. Some 20 billion events are collected. More than 200,000 charm decays are fully reconstructed. Data analysis in progress ( $\mathrm{July} 96$ ).

Journal papers NIM A324 (1993) 535, PL B371 (1996) 157, PL

B379 (1996) 292, and PRL 76 (1996) 364. More publications expected.

Related experiments FNAL-653, FNAL-687, FNAL-691, FNAL769, CERN-WA-082, CERN-WA-089

E-mail contact appel@fnal.gov, purohit@fnal.gov

WWW Home-page

http:/fnphyx-www.fnal.gov/experiments/e791/welcome.html

\section{FNAL-799}

(Proposed Jan 1989, Approved Jun 1989, Began data-taking Oct 1991, In progress)

SEARCH FOR THE DECAY $K_{L} \rightarrow \pi^{0} e^{+} e^{-}$

UCLA - K Arisaka, D Chen, S M Field, J R Jennings, J Kubic, D Roberts, W E Slater, $M$ Weaver

UC, SAN DIEGO - H G E Kobrak, E Potter, R A Swanson G A White

CHICAGO U - E C Blucher, G C Bown, R A Briere, S Bright,

E Cheu, G E Graham, J Graham, D Harris, R S Kessler,

G D Makoff, K Mcfarland, V Prasad, G B Quinn, A J Roodman,

B Schwingenheuer, P S Shawhan, N Solomey, Y W Wah

( $\checkmark$ Spokesperson), B D Winstein, R Winston, E D Zimmerman
COLORADO U - A R Barker, D Fillmore, P L Mikelsons,

U Nauenberg, J-Y Wu

ELMHURST COLL - E C Swaliow

FERMILAB - R Ben-David, G J Bock, S R Childress,

R N Coleman, M B Crisler, 3 Enagonio, R L Ford, Y B Hsiung,

D A Jensen, T Kobilarcik, H Nguyen, V O'Dell, R Pordes,

S A Pordes, D R Pushka, E J Ramberg, R E Ray, $J_{r}$,

A I Ronzhin, K C Stanfield, R S Tschirhart, K Vaziri,

H B White, J Whitmore

ILLINOIS U, URBANA - E Collins, G D Gollin

OSAKA U - K Hanagaki, M Hazumi, T Hazumi, S Hidaka,

F Kato, T Nakaya, M Sadamoto, M Sogo, M Yagi, T Yamanaka ( $\checkmark$ Spokesperson)

RICE U - J Barnes, Ii, M D Corcoran, B P Padley

RUTGERS U - I F M Albuquerque, J W Belz, P M Haas,

E Halkiadakis, W P Hogan, S K Kim, A Lath, J N Matthews,

S S Myung, G Ping, S R Schnetzer, S V Somalwar, R L Stone, R Tesarek, G B Thomson, Y Zou

VIRGINIA $U-M$ W Arenton, B B Cox, H Duan, K HaganIngram, V Jejer, S Ledovskoy, A P Mcmanus, K S Nelson WISCONSIN U - A Alavi-Harati, T Alexopoulos, A R Erwin Accelerator FNAL-TEV Detector Spectrometer, Calorimeter Reactions

$\begin{array}{lc}p \text { nucleon } \rightarrow \Lambda \mathrm{X} & 800 \mathrm{GeV} / c \\ p \text { nucleon } \rightarrow \bar{\Lambda} \mathrm{X} & " \\ K_{L} \rightarrow \pi^{0} e^{+} e^{-} & 50-150 \mathrm{GeV} / c \\ K_{L} \rightarrow \pi^{0} \mu^{+} \mu^{-} & " \\ K_{L} \rightarrow \pi^{0} \nu_{e} \bar{\nu}_{e} & " \\ K_{L} \rightarrow e^{+} e^{-} e^{+} e^{-} & " \\ K_{L} \rightarrow e^{+} e^{-} \gamma \gamma & " \\ K_{L} \rightarrow \mu^{+} \mu^{-} \gamma & " \\ K_{L} \rightarrow \pi^{0} \pi^{0} \gamma & " \\ \pi^{0} \rightarrow e^{+} e^{-} & " \\ \pi^{0} \rightarrow e^{+} \mu^{-} & " \\ \pi^{0} \rightarrow e^{-} \mu^{+} & "\end{array}$

Particles studied $K_{L}, \pi^{0}, \Lambda$

Brief description The goal is to use rare $K_{L}$ decays as a probe for the $C P$ violation. Phase-I modifics the existing apparatus of FNAL-731 to haxdle increased $K_{L}$ fux and an extended decay region, and to provide a better muon identification. Studies various multibody rare $K_{L}$ decays, and $\pi^{0}$ decays. PhaseI completed data taking in January 92 . Phase-II uses a new beamline and a new detector including a new Csl calorimeter to improve the rejection of $K_{L} \rightarrow e^{+} e^{-\gamma \gamma}$ background from the $K_{L} \rightarrow \pi^{0} e^{+} e^{-}$signal. It also uses a new transition radiation detector (TRD) to achieve a better $\pi / e$ rejection. The sensitivity is expected to approach the $10^{-11}$ level for many rarc $K_{L}$ decays. See also the closely related FNAL-832 experiment. Phase-II is now taking data (July 96).

Journal papers PRL 71 (1993) 31, PRL 71 (1993) 3914, PRL 71 (1993) 3918, PL B320 (1994) 407, PL B338 (1994) 403, PRL 72 (1994) 3000, PRL 72 (1994) 3758, PRL 73 (1994) 2169, PR D50 (1994) 1874, and PRL 74 (1995) 3323.

Related experiments FNAL-731, FNAL-773, FNAL-832

E-mail contact wah@hep.uchicago.edu, taku@fnal.gov

\section{FNAL-800}

(Proposed Mar 1988, Approved Oct 1988, Completed datataking Jan 1992)

\section{MEASUREMENT OF THE MAGNETIC MOMENT OF} THE $\Omega^{-}$HYPERON

ARIZONA U - D K Fein, E B James, K A Johns (Spokesperson) DEPAUW U - V A Decarlo, P M Tynan

FERMILAB - G Allan, R A Rameika (Spokesperson)

MICIIIGAN U -. Y T Gao, M J Longo

MINNESOTA U - J A Ayala-Mercado, P M Border, D P Ciampa, G M Gughielmo, K J Heller, J Jalilian-Marian, N B Wallace,

D M Woods

Accelerator FNAL-TEV Detector Spectrometer 


\section{SUMMARIES OF FERMILAB EXPERIMENTS}

\author{
Reactions \\ $p \mathrm{Be} \rightarrow \Omega^{-} \mathrm{X}$ \\ $\Lambda \mathrm{Cu} \rightarrow \Omega^{-} \mathrm{X}$ \\ $\Lambda \mathrm{Cu} \rightarrow \Xi^{-} \mathrm{X}$ \\ $\Xi^{0} \mathrm{Cu} \rightarrow \Omega^{-} \mathrm{X}$ \\ $\Xi^{0} \mathrm{Cu} \rightarrow \Xi^{-} \mathrm{X}$ \\ $800 \mathrm{GeV} / \mathrm{c}$ \\ $300-500 \mathrm{GeV} / \mathrm{c}$ \\ $"$ \\ Particles studied $\Omega^{-}, \Xi^{-}$ \\ Brief description An extension of FNAL-756. Uses two methods \\ to produce polarized $\Omega$ 's. The spin transfer method uses 800 - \\ $\mathrm{GeV}$ protons to produce a secondary neutral beam of polarized \\ $\Lambda$ 's and $\Xi^{0}$ 's, which is then used to produce a tertiary beam \\ of polarized $\Omega$ 's at $0 \mathrm{mr}$. The neutral production method uses \\ a secondary beam of unpolarized $\Lambda$ 's and $\Xi^{0}$ 's incident at a \\ production angle to produce polarized $\Omega$ 's. The spectrometer \\ consists of a set of silicon strip detectors and a set of multiwire \\ proportional chambers. \\ Journal papers PRL 74 (1985) 3732. \\ Related experiments FNAL-756 \\ E-mail contact johns@fnal.gov, rameika@fnal.gov
}

\section{FNAL-803}

(Proposed 1990, 1993, Approved Nov 1993, In preparation)

$\nu_{\mu}$ TO $\nu_{\tau}$ OSCILLATIONS

COSMOS COLLABORATION

AICHI U OF EDUCATION - K Kodama, N Ushida

ATHENS U - G S Tzanakos

UC, DAVIS - V Paolone, P M Yager

CHANGWON NATIONAL U - C H HIahn

CHONNAM NATIONAL U $-J Y$ Kim

COLUMBIA U - J M Conrad, M H Shacvitz, E G Stern

FERMILAB -. V D Bogert, T G Carter, G M Koizumi,

B G Lundberg, $\Lambda$ J Malensek, J G Morfin, R A Rameika

GIFU U - K Nakazawa, $S$ Tasaka

GYEONGSANG NATIONAL U .. I G Park, J S Song

FIROSAKI U - S Kuramata

ILLINOIS TECH - R A Burnstein. H A Rubin

INDIANA U - C Bower, R M Heinz, L Miller, S Mufson, J Musser

KANSAS STATE U -- T A Bolton, S McPeek, D L Naples,

N W Reay ( $\checkmark$ Spokesperson), R A Sidwell. E Smith,

N R Stanton, D M Woods, S Yoshida

KINKI U, OSAKA - M Chikawa

KOBE $U$.. S Aoki, T Hara

KOREA INST SCI - J K Kim

KOREA U $-\mathrm{J} S \mathrm{~K}$ ang, C O Kim

MICHIGAN U - R C Ball, S Coutu, K Green, D Levin,

J Matthews. S McKee, D F Nitz, S Nutter, J K Riles, B P Roe,

G Tarle, R P Thun, J C Vander Velde, C Weaverdyck

MINNESOTA U .. J K Nelson, R W Rusack, V M Singh

MOSCOW, ITEP - A Asratyan, M Balats, G Davidenko,

A Dolgolenko, G Dzyubenko, A Evdokimov, A Gerasimov,

V S Kaftanov, M A Kubantsev, V K Semyachkin, A L Sitnikov, $\checkmark$ Verebryusov, V E Vishnyakov

NAGOYA INST TECH -. Y Isokane, Y Tsuncoka

NAGOYA U - K Hoshino, H Kitamura, M Kobayashi,

M Miyanishi, M Nakamura, Y Nakamura, S Nakanishi, K Niu,

K Niwa. M Nomura, K Saito, II Tajima, K Teraoka

OKAYAMA U - K Moriyama, II Shibata

OSAKA CITY U - $T$ Okusawa, M Teranaka, T Tominaga,

$T$ Watanabe, $T$ Yoshida

OSAKA PREFECTURE U - H Okabe, J Yokota

OSAKA U OF COMMERCE - G Fujioka, Y Takahashi

SEOUL NATIONAL U - J W KIm

SOAI U - O Kusumoto

SOU'TII CAROLINA U - F 'T Avignone, A Kulik, C Rosenfeld

TECHNION - J Goldberg

TOHO U -- M Adachi, M Kazuno, Y Kobayashi, E Niu, S Ono, H Shibuya, Y Umezawa

TUFT'S U -- T Kafka, A Napier, W P Oliver, J Schneps

UCLA - M Atac, D B Cline, W Hong, J Park, J M Rhoades,

J K WOO

UTSUNOMIYA U - Y Sato, I Tezuka

YOKOHAMA NATTONAL U - Y Maeda
Accelerator FNAL Detector Emulsion, Spectrometer

Reactions

$\nu_{\tau}$ nucleon $\rightarrow \tau \mathrm{X}$

Particles studied $\nu_{\mu}, \nu_{\tau}$

Brief description This is a short-baseline neutrino oscillation experiment which searches for the $\nu_{\mu} \rightarrow \nu_{\tau}, \nu_{e} \rightarrow \nu_{\tau}$, and $\nu_{\mu} \rightarrow \nu_{e}$ transitions. Uses the COSMOS detector, a hybrid emulsion - electronic spectrometer sensitive to uitra-small mixing angles for neutrino mass differences in the range larger than $0.1 \mathrm{eV}^{2}$. Resides on a wide band, $10-70 \mathrm{GeV}$, neutrino beam from Fermilab's new Main Injector. In preparation (July 96).

Related experiments FNAL-531, CERN-WA-095, CERN-WA-096

E-mail contact reay@hep.phys.ksu.edu, reay@inal,gov WWW Home-page

http://roo.physics.lsa.umich.edu/www/e803/e803.html

\section{FNAL-811}

(Proposed Mar 1991, Approved Jul 1992, Began data-taking Jan 1996, Completed data-taking Feb 1996)

\section{$\bar{p} p$ ELASTIC SCATTERING}

CERN - R DeSalvo, M R Mondardini

CORNELL U - C Avila, C M Guss, J Orear ( $\checkmark$ Spokesperson)

FERMILAB - W F Baker, D P Eartly, H Jostlein, S M Pruss,

R Rubinstein, F Turkot

Accelerator FNAL-COLLIDER Detector Scintillator

\section{Reactrons}

$\bar{p} p \rightarrow \bar{p} p$

$1800 \mathrm{GeV}\left(\mathrm{E}_{\mathrm{cm}}\right)$

Brief description The detector is a solid bundle of scintillating fibers. The fibers are parallel to the beam, inside the beam pipe. Can be remotely moved close to the beam. Measures $x$ and $y$ coordinates of scattered protons to 50-micron accuracy. Scattering angles are small enough to observe Coulomb interference and to use the optical theorem to get total cross-section. Data analysis in progress (July 96).

Journal papers NIM A323 (1992) 419, NP (PROC SUPPL) $25 B$ (1992) 261, and NP (PROC SUPPL) 25B (1992) 294.

Related experiments FNAL-710

E-mail contact jo@lns62.1ns.cornell.edu WWW Home-page http://fne811.fnal.gov:8000/

\section{FNAL-815}

(Proposed Oct 1990, Approved Jul 1992, Began data-taking May 1996 , In progress)

PRECISION MEASUREMENTS OF NEUTRINO NEUTRAL CURRENT INTERACTIONS USING A SIGNSELECTED BEAM

NU'TEV COLLABORATION

ADELPHI U .- R V Stciner

CINCINNATI U - R Johnson, L P Perera, $G W$ Troha, M Vakili

COLUMBIA U .- J M Conrad, $R$ Gall, J H Kim, S Koutsoliotas, S Lammers, C Mcinulty, A Romosan, P C Rowson, M H Shaevitz ( $\checkmark$ Spokesperson), P Spentzouris, E G Stern A Vaitaitis

FERMILAB - R H Bernstein ( $\checkmark$ Spokesperson), L Bugel, G Koizumi, M J Lamm, W L Marsh, K S McFarland, J Yu

KANSAS STATE U .- T A Bolton, J Goldman, S McPeek, D L Naples, J Norris

NORTHWESTERN U - D A Buchholz, L Debarbaro.

II M Schellman, $G$ Zeller

OREGON U - J E Brau, R B Drucker, R E Frey, D Mason ROCHESTER U - P S Auchincloss, S Avvakumov, $\Lambda$ Bodek,

H S Budd. P Debarbaro, D Harris, W K Sakumoto, U K Yang XAVIER U .. P Nienaber

Accelerator FNAL-TEV Detector LAB-E 


\section{SUMMARIES OF FERMILAB EXPERIMENTS}

Reactions

$$
\nu n \rightarrow \mu-\mathrm{X}
$$

$\nu n \rightarrow \nu \mathrm{X}$

$250 \mathrm{GeV}\left(\mathrm{E}_{\text {lab }}\right)$

"

Particles studied $\nu$

Brief description The primary physics goal is to measure $\sin ^{2} \theta_{W}$ to a precision of $\pm(0.002-0.003)$. The high precision is achieved by making use of a new high-intensity sign-selected neutrino beam. The new beam design permits clean separation of $\nu$ from $\bar{\nu}$ while providing enough intensity to maintain small statistical errors. Other goals include the study of the QCD scale parameter $\Lambda$, the charm mass, the CKM matrix element $V_{c d}$, and the effects of the strange quark sea and charm quark sea on proton. Approved for a two-year run. Taking data (July 96)

Related experiments FNAL-744, FNAL-770

E-mail contact shaevitz@nevis.columbia.edu, rhbob@fnal.gov WWW Home-page http://cordelia.fnal.gov/NuTeV.html

\section{FNAL-831}

(Proposed Oct 1990, Approved Dec 1992, Iri preparation)

HIGH STATISTICS STUDY OF STATES CONTAINING HEAVY QUARKS USING THE WIDE BAND PHOTON BEAM

FOCUS COLLABORATION

UC. DAVIS - J Link, V Paolone, P M Yager

RIO DE JANEIRO, CBPF - A L Barbosa, I Bediaga,

J C Dos Anjos. C Gobel, G Guedes, I M Pepe, A Reis Correa, F Siman

MEXICO, XPN - S Carrillo, E Casimiro, G Herrera-Corral,

H Mendez, C Uribe

COLORADO U - L Cinquini, J P Cumalat ( $\checkmark$ Spokesperson), E S Erdos, B O'Reilly, E Vaandering

FERMILAB - J N Butler, H W K Cheung, S Cihangir, I Gaines, P H Garbincius, L A Garren, S A Gourlay, D I Harding, P H Kasper, $A$ E Kreymer, P L G Lebrun, S Shukla

FRASCA'II - S Bianco, F Fabbri, M Giardoni, L Passamonti,

V Russo. S Sarwar, A Zallo

ILLINOIS U, URBANA - C Cawlfield, F D Cogswell, R Gardner, K S Park, L Peak, A M Rahimi, J E Wiss

KOREA U - B G Cheon, Y S Chung, J S Kang, K Y Kim, K B Lee, S S Myung

INFN, MILAN - M Boschini, P D'Angelo, P Inzani, S Málvezzi, D Menasce. E Meroni, L Moroni ( $\checkmark$ Spokesperson), D Fedrini,

L Perasso, F P Prelz, A Sala-Grabar, S Sala

MILAN U - G Alimonti, G Bellini, B Caccianiga, M Dicorato, P Dini, M G Giammarchi, F Leveraro, L Milazzo

NOR'TH CAROLINA U - 'T F Davenport, III

NOTIRE DAME U - J M Bishop, N M Cason, J M Losecco, W D Shephard

PAVIA U - V Arena, O Barnaba, G Boca, G Bonomi, S Bricola, C Casella, E D'Uscio, G Gianini, E Imbres, G Juvino, G Liquori, T Locatelli, M Marchesotti, S P Ratti, C M Riccardi, P Torre, F Vercellati, L Viola, P Vitulo

PUEBLA $U$, MEXICO - E Cantoral, A Fernandez, S Hernandez, G Munoz, J Ramirez

PUERTO RICO U, MAYAGUEZ - J Alemar, A M Lopez,

L Mende\%, A Mirles, E Montiel, H Mourad, J Ramirez,

C Rivera, W Rolke, Y L Zhang

SOU'H CAROLINA U - N Copty, W Johns, M V Purohit, $\mathrm{J}$ R Wilson

T'ENNESSEE U .- G T Condo, K Danyo-Blackett, T Handler

VANOERBII' U -- J W Cao, M Hosack, M Nehring, P D Sheldon.

M \& Webster

WISCONSIN U - M C Sheaff, K Stenson

Accelerator FNAL-'TEV Detector Spectrometer

Reactions

$\gamma$ nucleus $\rightarrow$ charm meson $X$

$\gamma$ nucleus $\rightarrow$ charm baryon $\mathrm{X}$

$$
\underset{\sim}{250 \mathrm{GeV} / c}\left(\mathrm{P}_{\mathrm{lab}}\right)
$$

Partzcles studied $\psi$ (unspec), charm

Brief description Continues studies of FNAL-687. Uses

bremsstrahlung photons from a wide band $250 \mathrm{GeV}( \pm 15 \%)$

electron beam, a new large-aperture multiparticle spectrometer, a beryllium target, and a silicon microstrip decay-vertex detector. Studies the photoproduction and decay of heavy quarks. Being installed (July 96).

Related experiments FNAL-687

E-mail contact jcumalat@fnal.gov, moroni@fnal.gov WWW Home-page http://da831.fnal.gov/

\section{FNAL-832}

(Proposed Oct 1990, Approved Jun 1992, Began data-taking Jul 1996, In progress)

\section{SEARCH FOR DIRECT $C P$ VIOLATION IN THE $2 \pi$ DECAYS OF THE NEUTRAL KAON}

KTEV COLLABORATION

UCLA - K Arisaka, S M Field, J R Jennings, J Kubic, W E Slater UC, SAN DIEGO - H G E Kobrak, E Potter, R A Swanson,

G A White

CHYCAGO U - E C Blucher, G C Bown, S Bright, E Cheu,

G E Graham, J Graham, R S Kessler, V Prasad, G B Quinn.

A J Roodman, P S Shawhan, N Solomey, Y W Wah,

$B D$ Winstein ( $\checkmark$ Spokesperson), $R$ Winston, E D Zimmerman

COLORADO U - A R Barker, D Fillmore, P L Mikelsons,

U Nauenberg, $\mathrm{J} X \mathrm{Wu}$

ELMHURST COLL - E C Swallow

FERMILAB - L, Bellantoni, R Ben-David, G J Bock,

$S R$ Childress, $R$ N Coleman, $M$ B Crisler, R L Ford,

$X$ B Isiung ( $\checkmark$ Spokesperson), D Jensen, T Kobilarcik,

T Nakaya, II Nguyen, V O'Dell, M Pang, $R$ Pordes, S A Pordes,

D R Pushka, E I Ramberg, R E Ray, Jr, A I Ronzhin,

$P$ Shanahan, $K$ C Stanfield, $R$ S Tschirhart, K Vaziri,

H B White, J Whitmore

OSAKA U - K Hanagaki, M Hazumi, T Hazumi, S Hidaka,

F Kato, Y Matsumiya, M Sadamoto, M Sogo, M Takita,

'T Tsuji, M Yagi, T Yamanaka

RICE U - A Belianvance, M D Corcoran, B P Padley

RUTGERS U - I F M Albuquerque, S Averitte, I W Belz,

E Halkiadakis, $A$ Lath, S R Schnetzer, S V Somalwar,

R. L Stone, R Tesarek, G B Thomson

VIRGINIA U - M W Arenton, B B Cox, K Hagan-Ingram,

V Jejer, $S$ Ledovskoy, A P Mcmanus, K S Nelson

WISCONSIN U - A Alavi-Harati, $\Upsilon$ Alexopoulos, A R Erwin

Accelerator FNAL-TEV Detector Spectrometer, Calorimeter

Reactions

$$
\begin{aligned}
& K_{L} \rightarrow \pi^{+} \pi^{-} \\
& K_{L} \rightarrow \pi^{0} \pi^{0} \\
& K_{L} \rightarrow \pi^{+} \pi^{-} \gamma \\
& K_{L} \rightarrow \pi^{0} \gamma \gamma \\
& K_{L} \rightarrow \pi^{0} \nu \bar{\nu} \\
& K_{S} \rightarrow \pi^{+} \pi^{-} \\
& K_{S} \rightarrow \pi^{0} \pi^{0} \\
& K_{S} \rightarrow \pi^{+} \pi^{-} \gamma
\end{aligned}
$$

$30-160 \mathrm{GeV} / \mathrm{c}\left(\mathrm{P}_{\mathrm{lab}}\right)$

"

Particles studzed $K_{L}, K_{S}$

Bref description Measures the direct $C P$ violation parameter $\operatorname{Re}\left(\epsilon^{\prime} / \epsilon\right)$ to the precision of $10^{-4}$. The new neutral kaon beam facility, $\mathrm{KTeV}$, is constructed to give five times more flux, with reduced muon background and accidental rate.

The apparatus gives twice as long a decay region, higher rate capability, and more hermetic photon veto coverage against. the $3 \pi^{\circ}$ background. The position and encrgy resolution of the electromagnetic calorimeter (CsI) for elcctron and photon are improved. Uses a beryllium target. Taking data (July 96).

Journal papers NIM A367 (1995) 252, and NIM A368 (1996) 653

Related experiments FNAL-731, $773, \cdot \cdot 799$, CERN-NA-048

E-mail contact bruce@fnal.gov, brucc@hep.uchicago.edu. hsiung@fnal.gov

WWW Home-page

http://fnphyx-www.fnal.gov/experiments/ktev/ktev.html 


\section{SUMMARIES OF FERMILAB EXPERIMENTS}

\section{FNAL-835}

(Proposed 1992, Approved Dec 1993, In preparation)

\section{STUDY OF CHARMONIUM SPECTROSCOPY IN PROTON-ANTIPROTON ANNIHILATION}

CHARMONIUM COLLABORATION

UC, IRVINE - K E Gollwitzer, M A Mandelkern, J Schultz, M Thompson, G Zioulas

FERMILAB - M D Church, A A Hahn, W L Marsh,

J Peoples, Jr, S A Pordes, P A Rapidis, J Streets, S Werkema

FERRARA U - M Ambrogiani, W Baldini, D Bettoni,

$M$ Bombanoti, D Bonsi, R Calabrese, P Dalpiaz, E Luppi,

R Mussa, M Savrie, G Stancari

INFN, GENOA - A Buzzo, M Lovetere, M Macri, M M Marinelli,

M Pallavicini, C Patrignani, M G Pia, E Robutti, A Santroni

NORTHWES'TERN U - X L Fan, S Jin, P Maas, T K Pedlar,

J L Rosen, K K Seth

TURIN U - G Borreani, $\mathbb{R}$ Cester ( $\checkmark$ Spokesperson), F Marchetto,

E A Menichetti, N M Pastrone

Accelerator FNAL Detector Tracking system, Calorimeter

Reactions

$$
\begin{array}{lc}
\bar{p} p \rightarrow \overline{\text { charm }} \text { charm } & 3-7 \mathrm{GcV} / c\left(\mathrm{P}_{\mathrm{lab}}\right) \\
\bar{p} p \rightarrow e^{+} e^{-} & " \\
\bar{p} p \rightarrow \gamma^{\prime} \mathrm{s} & " \\
\bar{p} p \rightarrow \bar{p} p & " \\
\bar{p} p \rightarrow \phi \phi &
\end{array}
$$

Particles studied $\eta_{c}(1 S), \eta_{c}(2 S), h_{c}(1 P), \chi_{c 0}(1 P), D$

Brief description Continues the program of the FNAL-760 experiment in order to complete the study of the masses, widths and branching fractions of charmonium states. Adds a search for cryptoexotics and a study of $\mathrm{J} / \psi$ formation in nuclear matter. Improvements in the apparatus include: increased density of the internal target, better charged particle detection, an upgrade in the central calorimetry and a new data acquisition architecture. This is an Accumulator Ring experiment. Being installed ( $y_{\text {uly }} 96$ ).

Related experiments FNAL-760

E-mail contact cester@to.infn.it, cester@fnal.gov WWW Home-page htp://www-e835.fnal.gov/

\section{FNAL-866}

(Proposed Scp 1992, Approved Dec 1992, In preparation)

MEASUREMENT OF THE RATIO OF ANTIQUARK DISTRIBUTIONS $\bar{d}(x) / \bar{u}(x)$ IN THE PROTON

NUSEA COLLABORATION

ABILENE CHRISTIAN U -- L D Iscnhower, M E Sadler, R S 'Towell, D Wise

ARGONNE - K G Bailey, D F Geesaman, H E Jackson, Jr

C Jones, S Kaufman, R Kowalczyk, N Makins, T O'Neill,

D H Potterveld, J Reinhold, B Zcidman, $\triangle$ Zeuli

FERMILAB - CN Brown, $W €$ Cooper, $C S$ Mishra

GEORGIA STATE U - X C He, W M Lee, G Petitt

ILLINOIS TECH - D M Kaplan

LOS ALAMOS - M L Brooks, T A Carey, F Federspeil,

G ' $\mathrm{T}$ Garvey, D M Jansen, D M Lee, M J Leitch,

J B McClelland, P L McGaughey ( $\checkmark$ Spokesperson), C L Morris,

$\mathrm{J} M$ Moss, B K Park, J C Peng. W Sondheim, $T$ Thompson LOUISIANA STATE U - P N Kirk, Y C Wang, Z F Wang

NEW MEXICO STATE U - M Beddo, G Burleson, T H Chang,

J Ylaas, G S Kylc, V Papavassilio, Z M Wans

OAK RIDGE - T Awes, H Kim, F Obenshain, F Plasil, S Saini,

P Stankus, G $R$ Young

'TEXAS A AND M - C A Gagliardi, E Hawker, R E Tribble

VALPARAISO U, INDIANA - D D Koetke, P Nord. S Stanislaus

Accelerator FNAL-TEV Detector Spectrometer

Reactions

$p$ nucleon

$800 \mathrm{GeV}\left(\mathrm{T}_{\mathrm{lab}}\right)$

Particles studied
Brief description The experiment is a precision measurement of Drell-Yan yields from hydrogen and deuterium. The ratio of these yields can be used to infer the ratio $\bar{u}(x) / \bar{d}(x)$ in the proton, over the $x$ interval between 0.03 and 0.3 . Measures also the $\mathrm{J} / \psi, \psi^{\prime}, \Upsilon, \Upsilon^{\prime}$, and $\Upsilon^{\prime \prime}$ yields from both targets. Uses the Magnetic Dilepton Spectrometer, with 3 dipoles, 3 stations of wire chambers, 1 station with prop-tubes, 4 hodoscope stations, and high rate capability with better than $100 \mathrm{MeV}$ resolution at the $J / \psi$. Beam produces $10^{12}$ protons/spill. Targets are LH and LD. Being installed ( $\mathrm{July} 96$ ).

Related experiments FNAL-772, CERN-NA-051

E-mail contact plm@lanl.gov

WWW Home-page http://p2hp2.lanl.gov/e866/e866.html

\section{FNAL-868}

(Proposed Sep 1992, Approved Mar 1993, Began data-taking Apr 1995, Completed data-taking Jul 1995)

\section{SEARCH FOR ANTIPROTON DECAY AT THE AN-} TIPROTON ACCUMULATOR

\section{APEX COLLABORATION}

UCZA - C D Buchanan, B Corbin, M A Lindgren, T Muller FERMIL $A B-\underline{S}$ Geer ( $\checkmark$ Spokesperson), J P Marriner,

M Martens, R E Ray, Jr, J Streets, W C Wester

MICHIGAN U - H R Gustafson

NEBRASKA U - M J Hu, G R Snow

PENN STATE U - T A Armstrong

Accelerator FNAL-TEV Detector Calorimeter

Reactions

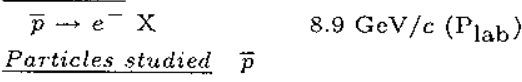

Brief description The detector consists of a calorimeter, fiber tracker, pre-radiator, DEDX, and vetos. Uses the Antiproton Accumulator facility. Data analysis in progress (July 96).

Related experiments FNAL-861

E-mail contact sgeer@fnal.gov

WWW Home-page http://fnapxl.fnal.gov/

\section{FNAL-871}

(Proposed Oct 1993, Approved Jul 1994, In preparation)

SEARCH FOR CP VIOLATION IN THE DECAYS OF $\Xi^{-} / \bar{\Xi}^{+}$AND $\Lambda / \bar{\Lambda}$ HYPERONS

HYPERCP COLLABORATION

TAIWAN, INST PHYS $-A$ Chan, $X C$ Chen, $K C$ Cheng, $C$ L Ho, $M$ Huang, S C Lee, P K Teng

SOUTH ALABA.MA U - R K Clark, C M Jenkins

LBL \& UC, BERKELEX - W S Choong, R Fuzesy, G Gidal.

P Gu, K B Luk ( $\sqrt{ }$ Spokesperson), B Turko

FERMILAB - T Carter, $\mathrm{M}$ Crisler, C C James, J $T$ Volk

GUANAJUATO U - J Felix-Valdez, G Moreno, M A Sosa Aquino

ILLINOIS TECH - $S$ Biedron, $R$ A Burnstein, J A Drapala,

D M Kaplan, L M Lederman, W Luebke, A Ozturk, H A Rubin,

D Sowinski, C White, $S$ White

LAUSANNE U - N Leros, J-P Perroud

MICHIGAN U H R Gustafson, M J Longo

VIRGINIA U - K Bodoor, S Conetti, E C Dukes

( $\checkmark$ Spokesperson), C Durandet, K S Nelson, D Rajaram,

N Saleh. H-G Zhu

Accelerator FNAL-TEV Detector Spectrometer, Spectrometer

Reactions

$$
\begin{array}{lc}
p \text { nucleon } \rightarrow \Xi^{-} \mathrm{X} & 150 \mathrm{GeV} / c \\
p \text { nucleon } \rightarrow \Xi^{+} \mathrm{X} & "
\end{array}
$$

Particles studied $\Xi^{-}, \bar{\Xi}^{+}, \Omega^{-}, \bar{\Omega}^{+}$

Brief description Unpolarized $\Xi^{--}$and $\bar{\Xi}^{+}$hyperons are produced by protons and momentum selected by a magnetic 


\section{SUMMARIES OF FERMILAB EXPERIMENTS}

channel. The decay sequences $\Xi^{-} \rightarrow \Lambda \pi^{-}, \Lambda \rightarrow p \pi^{-}$, and $\bar{\Xi}^{+} \rightarrow \bar{\Lambda} \pi^{+}, \bar{\Lambda} \rightarrow \bar{p} \pi^{+}$, are detected with a wire chamber spectrometcr with high-rate capability. By studying the angular distribution of $p(\bar{p})$ with respect to the helicity axis in the $\Lambda$ $(\bar{\Lambda})$ rest frame, the decay parameter $\alpha$ ( $\alpha$-bar) can be extracted. The $C P$ symmetry is violated if there is any difference between the products $\left(\alpha_{\Lambda} \alpha_{\Xi}\right)$, and $\left(\alpha_{\bar{\Lambda}} \alpha_{\bar{\Xi}}\right)$. The apparatus, $60 \mathrm{~m}$ long, consists of a hyperon magnet, nine wire chamber stations, a momentum analyzing magnet, two planes of hodoscopes for timing and triggering purposes, and a hadronic calorimeter for triggering. A muon detection system at the rear of the spectrometer allows searches for rare and forbidden hyperon decays. Under construction (July 96).

E-mail contact luk@fnal.gov, dukes@fnal.gov WWW Home-page http://beautyl.lbl.gov/e871.html

\section{FNAL-872}

(Proposed Jan 1994, Approved Jun 1994, In preparation)

MEASUREMENT OF $r$ LEPTON PRODUCTION FROM THE PROCESS $\nu_{\tau}+N \rightarrow \tau+X$

E872 COLLABORATION

AICKI U OF EDUCATION -- K Kodama, N Ushida ATHENS U - N Giokaris, G S Tzanakos

UC, DAVIS - V Paolone ( $\checkmark$ Spokesperson), P M Yager

KANGWEON NATYONAL U - C H Hahn

CHONNAM NATIONAL U - J-Y Kim

FERMILAB -- B R Baller, D Boehnlien, W S Freeman,

B G Lundberg ( $\checkmark$ Spokesperson), J G Morfin, R A Rameika

G YEONGSANG NA'TIONAL U - S H Chung, I G Park, J-S Song

KOBE U - S Aoki, T Hara

KON-KUK U . J T Rhee

KOREAN NATIONAL EDUCATION U - S N Kim

MINNESOTA U P M Border, D P Ciampa, K J Heller,

R W Rusack, J Sielaff, J J Trammell, J O Wilcox

NAGOYA U - N Hashizume, K Hoshino, H Inuma, K Ito,

M Kobayashi, M Miyanishi, K Nakajima, M Nakamura, K Niwa,

N Nonaka, K Okada, K Saito, K Yamamori

OSAKA PRETECTURE U - H Okabe

PUSAN NATIONAL U - W H Chung

SOU'TH CAROLINA U - A Kulik, C Rosenfeld

TOHO U -- M Adachi, M Kazuno, Y Kobayashi, E Niu, S Ono,

H Shibuya, $Y$ Umezawa

TUFTS U - T Kafka, A Napier, W P Oliver, J Schneps,

$M$ Skender

UTSUNOMIYA U - $Y$ Sato, I Tezuka

WONKWANG U - S Y Bahk

Accelerator FNAL-TEV Detector Spectrometer

Brief description Measures the production of $\tau$ leptons in the charged-current interactions of $\tau$ neutrinos. The neutrinos are produced in a beam dump by the $800 \mathrm{GeV}$ proton beam, and interact in an emulsion target. The resulting $\tau$ leptons are subsequently detected in a high resolution hybrid emulsion spectrometer, providing a direct confirmation of the existence of the $\tau$ neutrino. Under construction (July 96).

Related experments FNAL-803, CERN-WA-095

E-mail contact paolone@fnal.gov, lundberg@fnal.gov WWW Home-page http://fn872.fnal.gov/

\section{FNAL-875}

(Proposed Jan 1995, Approved May 1995, In preparation)

LONG BASELINE NEUTRINO OSCILLATION EXPERIMEN'T

\section{MINOS COLLABORATION}

ARGONNE - I Ambats, D S Ayres ( $\checkmark$ Deputy Spokesperson), L J Balka, R E Blair, D A Crane, J W Dawson, T H Fields, M C Goodman, V I Guarino, N F Hill, D J Jankowski, E N May, $S$ Mrenna, $L E$ Price, $P$ Schoessow, $R$ V Seidlein, R Talaga, K M Thompson. J Thron, L R Turner
BOSTON COLL - T E Toohig

CAL TECH - B C Barish, D L Burke, J Hanson, N P Longley,

D G Michael, C W Peck

COLUMBIA U - Y Ho, W Lee

FERMILAB -- R II Bernstein, V D Bogert, D Coissart,

W S Freeman, J Hylen, D E Johnson, G M Koizumi,

G Mitselmakher, J G Morfin, F A Nezrick, S C O'Day,

R A Rameika, A L Read, W Smart, A Wehman, S Werkema,

C P Yun

BEIJING, IHEP - J Guo, T Hu, Y Lai, H Mao, B Shen, M Wang, $X$ Xia, Y Xic, W Yan

INDIANA U - C Bower, M Gebhard, R W Hatcher, R M Heinz, L Miller, $S$ Mufson, J A Musser

DUBNA - S Afanasjev, G D Alexeev, S Borodin, V Brudanin,

G Chelkov, Y A Gornushkia, M Ignatankio, N Khovanski

Z V Krumstein, S Olshevski, A B Sadovski, A Sisakian,

V Timofeev, L Tkatchev, A I Zinchenko

LIVERMORE - E Ables, R Bionta, R Carney, E P Hartouni,

$T$ Ladran, M Libkind, H Olsan, L Ott, E Parker, J Swan,

D Wright, C Wuest, T Yokota

LEBEDEV INST - V A Chechin, E P Kuznetesov, V A Tsarev

MINNESOTA U - T Berg, P M Border, T Chase, D P Ciampa,

H W J Courant, P Cushman, K J Heller, M L Marshak,

D E Maxam, J Meier, B Miller, J K Nelson, E A Peterson,

K Ruddick, R W Rusack, M II Schub

MOSCOW, I'TEP .. V Smotriaev, I 'Trostin

OAK RIDGE -. C L Britton, W Bryan, U Jagadish

OXFORD U - W W M Allison, C B Brooks, I H Cobb, S Cooper,

H Gallagher, A R Holmes, D H Perkins, D A Petyt, P D Shicld,

J Thomas, R L Wastie, N West

RUTHERFORD - G J Alner, R J Cotton, R Edgecock, G Grayer, P J Litchfield, G F Pearce

STANFORD U - G Irwin, S G Wojcicki ( $\checkmark$ Spokesperson)

SUSSEX U - J Byrne, P G Dawber, K Green, P G Harris,

D White

TEXAS U - K Lang

TEXAS A AND M - A D David, N Diaczenko, M Drew, H-

J 'Trost, R C Webb

TUFTS U - T Kafka, W A Mann, R H Milburn, A Napier,

W P Oliver, J Schneps

WESTERN WASHINGTON U - W L Barrett

Accelerator FNAL Detector Calorimeter

Particles studied $\nu$

Brief description The MINOS (Main Injector Neutrino Oscillation Search) collaboration proposes to conduct a search for $\nu_{\mu} \rightarrow \nu_{\tau}$ and $\nu_{\mu} \rightarrow \nu_{e}$ oscillations using a new wide band $(15 \mathrm{GeV}) \nu_{\mu}$ beam from the Main Injector with energies well above the $\tau$ production threshold. The oscillations will be detected by the comparison of signals in a 'near' detector at Fermilab and a 'far' detector situated $730 \mathrm{~km}$ away in the Soudan underground laboratory. A new 10 kton detector will be built at Soudan to allow the study of the oscillation parameters down to $\Delta\left(m^{2}\right)=0.002 \mathrm{eV}^{2}$ and $\sin ^{2}(2 \theta)=0.01$. The existing. much finer grained but smaller, SOUDAN-2 detector (see the UNDERGROUND-SOUDAN-2 experiment) will provide an independent check of any potential signal with $\sin ^{2}(2 \theta)>1 / 30$. In preparation (July 96 ).

Relaled experiments UNDERGROUND-ICARUS, KEK-362

E-mail contact sgweg@slac.stanford.edu, mcg@hep.anl.gov WWW Home-page

http://www.hep.anl.gov/NDK/Hypertext/numi.html

\section{FNAL-876}

(Proposed Jan 1995, Approved Aug 1995. Began data-taking Dec 1995. Completed data-laking Feb 1996)

\section{CDF HARD DIFFRACTION STUDIES}

CDF COLLABORATION

Accelerator FNAL-COLLIDER Detector CDF, Other

Reactions

$\bar{p} p \rightarrow \vec{p} \mathrm{X}$ 


\section{SUMMARIES OF FERMILAB EXPERIMENTS}

Brief description Studies hard and high mass diffraction as a part of the CDF QCD program. The physics goal is a better understanding of the nature of the pomeron's constituents. Detects quasielastically scattered (anti)protons with the precision track detectors (scintillating fiber hodoscopes backed up by a trigger counter), inserted in movable Roman Pots. The track measurement together with the vertex information from the CDF vertex detector can be used to select the high-mass diffractive events. The experiment is carried out in parallel with the other CDF studies, and with the participation of the full CDF Collaboration. Data analysis in progress (July 96). To learn more about the program, please contact the Spokesperson for the Hard Diffraction Group, Dr. Michael Albrow [Fermilab]

Related experiments CERN-UA-008, FNAL-741

E-mail contact albrow@fnal.gov

\section{Other FNAL Experiments}

Listed here are some other Fermilab experiments of interest to the particle physics community. Find more details about these projects online, in the SLAC's EXPERIMENTS database (sce p. 3), or contact the spokespersons. Check also a detailed description of the ongoing projects at the Lab in the annual report 'Fermilab Research Program - Workbook', or visit the Web site http://www.fnal.gov/faw/fermilab_at_work.html

FNAL-683 (Completed data-taking Jan 1992)

PHOTOPRODUCTION OF HIGH $p_{\perp}$ JETS

Spokesperson: Marjorie D. Corcoran [Rice U.]

E-mail contact corcoran@physics.rice.edu, corcoran@fnal.gov

FNAL-706 (Completed data-taking Jan 1992)

A COMPREHENSIVE STUDY OF DIRECT PHOTON PRODUCTION IN HADRON INDUCED COLLISIONS By E706 Collaboration

Spokesperson: Paul F. Slattery [Rochester U.]

E-mail contact slattery@urhep.pas.rochester.edu

FNAL-793 (In preparation)

EMULSION EXPOSURE TO $1000 \mathrm{GeV}$, OR HIGHEST ENERGY PROTONS

Spokesperson: Jere J. Lord [Washington U., Seattle]

E-manl contact lord@phys.washington.edu

FNAL-802 (Completed data-taking Dec 1991)

DEEP INELASTIC MUON INTERACTIONS WITH NUCLEAR TARGETS USING THE EMULSION TELESCOPE TECHNIQUE

Spokespersons: Lali Chattexjee, Dipak Ghosh [Jadavpur U.)

E-mail contact thornton@fnal.gov

FNAL-843 (Completed data-taking Jul 1991)

INTERACTIONS OF 50, 100, AND $490 \mathrm{GeV}$ MUONS WITH EMULSION NUCLEI

Spokesperson: C.O. Kim [Korea U.]
FNAL-853 (Completed data-taking Feb 1996)

TEST OF LOW INTENSITY EXTRACTION FROM THE TEVATRON USING CHANNELING IN A BENT CRYSTAL

Spokespersons: Gerry P. Jackson, C.Thornton Murphy [Fermilab] E-marl contact thornton@fral.gov, gpj@fnal.gov WWW Home-page http://calvin.fnal.gov/ sathyade/cex.html

FNAL-855 (Completed data-taking Dec 1991)

TEST BEAM REQUEST TO DIRECTLY MEASURE $d E / d x$ OF HIGH ENERGY MUONS FROM 150 TO 650 GeV/c IN THE MUON LABORATORY

Spokesperson: George R. KalbPeisch [Oklahoma U.]

E-mail contact grk@fnal.gov

FNAL-861 (Completed data-taking Oct 1992)

SEARCHING FOR ANTIPROTON DECAY AT THE FERMILAB ANTIPROTON SOURCE

By APEX Collaboration

Spokesperson: Steve Geer [Fermilab]

E-mail contact sgeer@fnald.fnal.gov

WWW Home-page http://fnapxl.fnal.gov/

FNAL-862 (In preparation)

DETECTION OF RELATIVISTIC ANTI-HYDROGEN ATOMS PRODUCED BY PAIR PRODUCTION WITH POSITRON CAPTURE

By ANTIHYDROGEN Collaboration

Spokesperson: David C. Christian [Fermilab]

E-mail contact dcc@fnal.gov

WWW Home-page

http://fnphyx-www.fnal.gov/experiments/e862/e862.html

FNAL-864 (Completed data-taking Feb 1996)

MINIMAX: A TEST / EXPERIMENT FOR THE FERMI LAB COLLIDER

Spokespersons: Cyrus C. Taylor [Case Western Reserve U.], James D. Bjorken [SLAC]

E-mail contact cct@po.cwru.edu, bjorken@slac.stanford.edu WWW Home-page http://fnmine.fnal.gov/

\section{FNAL Future Plans}

The $800-\mathrm{GeV}$ fixed target run will continue until early 1998. Typical intensities of $2.5 \times 10^{13}$ per Tevatron cycle are anticipated, with the experimental program including $\epsilon^{\prime} / \epsilon$, rare kaon decays, charm physics, $\sin ^{2} \theta_{W}$ from neutrino scattering, and observation of $\nu_{\tau}$. Completion of commissioning of the Main Injector and Recycler is expected by mid 1999 , when the upgraded CDF and Do detectors will start a run. The goal of the run is to deliver at least $2 \mathrm{fo}^{-1}$ to each of the detectors by 2003 . The peak luminosity goal is $2 \times 10^{32} \mathrm{~cm}^{-2} \mathrm{~s}^{-1}$. Construction of the NuMI facilities at Fermilab and Soudan (Minnesota) should begin in 1999, and the two neutrino oscillation experiments will start data taking uoon completion. Slow spill $120 \mathrm{GeV}$ protons from the Main Injector will be used for fixed target experiments concurrently with the 'levatron collider program. 
SUMMARIES OF FRASCATI EXPERIMENTS

\section{Selected DAPHNE Experiments}

\section{FRASCATI-DAPHNE-KLOE}

(Proposed 1992, Approved Mar 1993, In preparation)

\section{A LARGE GENERAL PURPOSE DETECTOR FOR $C P$} VIOLATION STUDIES IN $e^{+} e^{-}$ANNIHILATIONS AT $\phi$

\section{KLOE COLLABORATION}

BARI U \& INFN, BARI - O Erriquez, A Farilla, F Ruggieri

FRASCATI - A Andryakov, A Antonelli, M Antonelli, D Babusci,

R Baldini-Ferroli, S Bellucci, G Bencivenni, S Bertolucci,

C Bloise, F Bossi, A Calcaterra, P Campana, G Capon,

M Carboni, M Cordelli, R De Sangro, P De Simone, F Donno,

G Felici, M L Ferrer, G Finocchiaro, A Gaddi, E Gero,

W Grandegger, R Haydar, L Keeble, P Laurelli, A Martini,

S Miscetti, S Moccia, F Murtas, G P Murtas, E Pace, V Patera,

F Pelucchi, M Piccolo, P Santantonio, M Spinetti, P Valente,

$\mathrm{X} \mathrm{L}$ Wang, $\mathrm{S}$ Wolfie, A Zallo

FRASCATI \& SUNY, STONY BROOK - J Lee-Franzini

KARLSRUHE U - K Barth, A Denig, M Imhof, C Joram,

W Kluge, U von Hagel, $S$ Weseler, $R$ Wieser

LECCE U \& INFN, LECCE - G Cataldi, P Creti, V Elia,

E Gorini, F Grancagnolo, G F Palama, M Panareo,

M Primavera, S Spagnolo

NAPLES U, IFS \& INFN, NAPLES - A Aloisio, V Baturin,

N Cavallo, F Cevenini, G Chiefari, E Drago, D Fiore, C Gatto,

L Merola, M Napolitano, A Smilzo

COLUMBIA U - P M Tuts

PISA U \& INFN, PISA - V Bolognesi, F Cervelli, A Ferrari,

$M$ Incagli, $M$ M Massai, $G$ Venanzoni

ROME U \& INFN, ROME - F Anulli, C Bacci, M Barone,

H Beker, C Bini, L Bucci, R Caloi, M Ceru, G De Zorzi,

$G$ Di Cosimo, A Di Domenico, P Franzini ( $\checkmark$ Spokesperson),

P Gauzzi, S Giovanella, F Lacava, G Lanfranchi, A Michetti,

A Parri, M Passaseo, E Petrolo, M C Petrucci, L Pontecorvo,

E Valente, S Veneziano

ROME U, TORVERGATA - V Bocci, L Cerrito, S D'Angelo,

R Messi, L Paoluzi, E Pasqualucci

ROME, ISS - P Branchini, E Graziani, A Passeri, E Spiriti,

C Stanescu, L Tortora

SUNY, STONY BROOK - W Kim, M Pollack, R D Schamberger

TRIESTE U, ISI' FIS \& INFN, TRIESTE - G Barbiellini,

A Martinis, F Scuri

Accelerator FRASCATI-DAPHNE Detector KLOE

Particles studied $K_{S}, K_{L}$

Brief description Studies rare kaon decays, measures $\operatorname{Re}\left(\epsilon^{\prime} / \epsilon\right)$

to the precision of $10^{-4}$, analyzes other $C P$ violating parameters in the $K \bar{K}$ system. KLOE consists of a $2-\mathrm{m}$ diameter, $3.5-\mathrm{m}$ long belium-filled central drift chamber, a $4 \pi$ clectromagnetic calorimeter, and a $2.3-\mathrm{m}$ diameter, $4-\mathrm{m}$ long, $0.6 \mathrm{~T}$ superconducting coil. The beam pipe is made of $0.5 \mathrm{~mm}$ thick beryllium to minimize multiple scattering and energy loss for charged kaons. In preparation. Expected to run in 1997.

Journal papers PL B287 (1992) 259, NP (PROC SUPPL) $37 \mathrm{~A}$

(1994) 43, NIM A360 (1995) 48, NIM A360 (1995) 201, NIM

A367 (1995) 104, NIM A367 (1995) 108, NIM A354 (1995) 352, and NIM A368 (1996) 628.

E-maul contact paolo@lnf.infn.it

$W W W$ Home-page http://www.lnf.infn.it/kloc/kloedef.html

\section{Other DAPHNE Experiments}

Listed here is another interesting DAPHNE experiment. Find more details about this projects online, in the SLAC's EXPERIMENTS database (see p. 3), or contact the spokesperson.

FRASCATI-DAPHNE-FINUDA (In preparation)

FINUDA: A DETECTOR FOR NUCLEAR PHYSICS AT DATNE

By FINUDA Collaboration

Spokesperson: Tullio Bressani [Turin U.]

$\underline{E-m a i l ~ c o n t a c t}$ bressanioto.infn.it

WWW Home-page

http://www.lnf.infn.it/esperimenti/finuda/finuda.htm! 


\section{SUMMARIES OF ITEP (MOSCOW) EXPERIMENTS}

\section{Selected ITEP Experiments}

\section{ITEP-895}

(Proposed 1989, Approved 1989, Began data-taking 1991, In progress)

DETAILED STUDY OF SPECTRA OF A PARTICLES AND OF THE POLARIZATION IN THE NUCLEAR, FRAGMENTATION REGION IN HADRON-NUCLEON INTERACTIONS

LAMBDA-III COLLABORATION

MOSCOW, ITEP - V A Akimov, Y D Bayukov, I M Belyaev, M P Bezuglov, B M Bobchenko, E A Doroshkevich, S V Frolov, Y G Grishuk, Y V Kantserov, M M Katz, S M Kiselev,

Y V Korchagin, S V Kuleshov, L N Kuleshova, A I Kurzenkov,

A A Lebedev, G A Leksin ( $\checkmark$ Spokesperson), N A Pivnyuk,

N K Sergeev, V S Serov, S M Shuvalov, A V Smirnitsky

( $\checkmark$ Spokesperson), A V Stavinsky, V P Surin, A V Vlasov,

K G Voloshin, L S Vorobyev, A V Yumashev, B V Zagreev,

$\mathrm{V} \vee$ Zhurkin

KURCHATOV INST, MOSCOW - S L Fokin, M S Ippolitov,

A L Lebedev, V I Manko, G M Mgebrishvili, P I Shcherbachev, $M$ A Vasilyev

Accelerator ITEP Detector LAMBDAMETER

\section{Reactions}

$$
\begin{array}{lc}
p \mathrm{Al} \rightarrow \Lambda \mathrm{X} & 7.5 \mathrm{GeV} / c \\
p \mathrm{~Pb} \rightarrow \Lambda \mathrm{X} & " 1
\end{array}
$$

Brief description The kinetic energy range of the detector is 10 $300 \mathrm{MeV}$. Taking data

E-mail contact leksin@vitep2.itep.ru, asmirnitsky@vxitep.itep.ru

\section{ITEP-913}

(Proposed 1990, Approved 1990, Began data-taking 1990

Completed data-taking 1993)

STUDY OF THE INTERACTION OF LOW-ENERGY ANTIPROTONS WITH NUCLEI USING THE XENON BUBBLE CHAMBER DIANA

DIANA COLLABORATION

MOSCOW, ITEP - V V Barmin, V G Barylov, G V Davidenko,

A G Dolgolenko ( $\checkmark$ Spokesperson), V E Lukhmanov

V A Matveev, G S Mirosidi, V A Shebanov, N N Shishov,

B S Volkov, N K Zombkovskaya

OSLO U - K M Danielsen, ' $T$ Jacobsen

FRASCATI \& BUCHAREST, IAP - F Nichitiu, C Petrascu MOSCOW, INR -.. E S Golubeva, A S Ilyinov, I A Pshenichnov BERGEN U - K Myklebost, J M Olsen

FRASCATI $\cdots$ C Guaraldo

Accelerator I'TEP Detector HLBC-DIANA

Reactions

$\begin{array}{lc}\bar{p} \mathrm{Xe} \rightarrow K_{S} \mathrm{X} & <1 \mathrm{GeV} / c\left(\mathrm{P}_{\mathrm{lab}}\right) \\ \bar{p} \mathrm{Xe} \rightarrow \Lambda\left(\Sigma^{0}\right) \mathrm{X} & " \\ \bar{p} \mathrm{Xe} \rightarrow \Sigma^{0} \mathrm{X} & " \\ \bar{p} \mathrm{Xe} \rightarrow K_{S} K_{S} \mathrm{X} & " \\ \bar{p} \mathrm{Xe} \rightarrow K_{S} \Lambda\left(\Sigma^{0}\right) \mathrm{X} & " \\ \bar{p} \mathrm{Xe} \rightarrow K^{+} \Lambda\left(\Sigma^{0}\right) \mathrm{X} & " \\ \bar{p} \mathrm{Xe} \rightarrow K^{+} \Sigma^{+} \mathrm{X} & " \\ \bar{p} \mathrm{Xe} \rightarrow K^{+} \Sigma^{-} \mathrm{X} & " \\ \bar{p} \mathrm{Xe} \rightarrow K^{+} K^{+} \mathrm{X} & " \\ \bar{p} \mathrm{Xe} \rightarrow K_{S} K^{-} \mathrm{X} & " \\ \bar{p} \mathrm{Xe} \rightarrow K^{+} K^{-} \mathrm{X} & " \\ \bar{p} \mathrm{Xe} \rightarrow K_{S} \Sigma^{+} & " \\ \bar{p} \mathrm{Xe} \rightarrow K_{S} \Sigma^{-} & " \\ \bar{p} \mathrm{Xe} \rightarrow \omega \mathrm{X} & \end{array}$

Brief description Uses the 700-liter Xenon bubble chamber DIANA. Studies the production of strange particles and $\eta, \omega$ mesons in low-energy $\vec{p}$ Xe annihilations.

Journal papers YF 55 (1992) $1253=$ SJNP 55 (1992) 698, YF 55 (1992) $1268=$ SJNP 55 (1992) 705, NP A.556 (1993) 409, NP A558 (1993) 361c, YF 57 (1994) $1724=$ PAN 57 (1994) 1656, YF 57 (1994) $1905=$ PAN 57 (1994) 1835, and PL B370 (1996) 233.

E-mail contact dolgalenko@vxitep.itep.ru

ITEP-922

(Proposed 1992, Approved 1992, Began data-taking 1996, In progress)

SEARCH FOR $H$-PARTICLE AND RESONANT STATES IN $\Lambda A$ SYSTEM

MOSCOW, ITEP - E G Bogdanov, V S Demidov

( $\checkmark$ Spokesperson), N D Galanina, K E Gusev, N A Khaldeeva,

$\checkmark \mathrm{N}$ Markisov, $\Lambda$ A Nedosekin, V A Sadykov, M E Vishnevsky, M O Vlasova

Accelerator ITEP Detector Spectrometer

Reactions

$$
\begin{array}{ll}
n \text { nuclcus } \rightarrow \Lambda \Lambda \mathrm{X} & 4-9 \mathrm{GeV} / c\left(\mathrm{P}_{\mathrm{lab}}\right) \\
n \text { nucleus } \rightarrow \text { dibaryon }(S=-2) \mathrm{X} & "
\end{array}
$$

Brief description Search for the $H$-dibaryon through the $\pi^{-} p \Lambda$ decay channel. Taking data (July 96).

E-mail.contact demidov@vxitep.itep.ru

\section{Other ITEP Experiments}

Listed here are some other ITEP experiments of interest to the particle physics community. Find more details about these projects online, in the SLAC's EXPERIMENTS database (sce p. 3), or contact the spokespersons.

ITEP-832 (Taking data)

A TRACKING EXPERIMENT FOR STUDY OF DOUBLE BETA DECAY $X \mathrm{~N}^{136} \mathrm{Xe}$ and ${ }^{150} \mathrm{Nd}$

Spokespersons: V.A. Lyubimov, O.Ya. Zeldovich [Moscow, I'TEP]

E-mail contact lubimov@vxitep.itep.ru, zeldovich@vxitep.itep.ru

ITEP-853 (Completed data-taking 1991)

STUDY OF PIONIC NUCLEAR DEGREES OF FREEDOM IN $(\pi, \pi \pi)$ REACTIONS

Spokespersons: M.V. Kossov, G.A. Leksin [Moscow, I'TEP]

E-mail contact kossov@cebaf.gov, leksin@vitep2.itep.ru

ITEP-861 (Completed data-taking 1991)

SEARCH FOR ${ }^{76}$ Ge DOUBLE BETA DECAY

Spokesperson: I.V. Kirpichnikov [Moscow, ITEP]

E-mail contact kirpichnikov@vxitep.itep.ru

ITEP-865 (Completed data-taking 1992)

ANTIPROTON-NUCLEI ANNIHULATION CROSS-

SECTIONS WITH Be, C, Al, $\mathrm{Fe}, \mathrm{Cd}, \mathrm{Cu}$, AND Pb TARGETS AT $0.70,0.95,1.26,1.53,1.76$ AND $2.50 \mathrm{GeV} / c$

Spokesperson: Yu.B. Lepikhin [Moscow, ITEP]

E-mail contact smirnitsky@vxitep.itep.ru 


\section{SUMMARIES OF ITEP (MOSCOW) EXPERIMENTS}

ITEP-873 (Completed data-taking 1991)

HIGH-ENERGY CUMULATIVE PARTICLE PRODUCTION AT $10 \mathrm{GeV}$

Spokespersons: Yu.T. Kiselev, Yu.V. Terekhov [Moscow, ITEP]

E-mail contact $\quad$ yurikis@vxitep.itep.ru, yurikis@vxcern.cern.ch

ITEP-876 (Completed data-taking 1992)

SEARCH FOR ${ }^{94} \mathrm{Zr}$ AND ${ }^{96} \mathrm{Zr}$ DOUBLE BETA DECAYS IN PHOTOGRAPHIC EMULSION

Spokesperson: A.S. Barabash [Moscow, ITEP]

E-mail contact barabash@vxitep.itep.ru

YTEP-892 (Completed data-taking 1994)

STUDY OF ${ }^{4} \mathrm{He} p$ INTERACTIONS

Spokesperson: V.E. Grechko [Moscow, ITEP]

E-mail contact grechko@vitepl.itep.ru

ITEP-894 (Completed data-taking 1991)

QUASIPARTICLE VELOCITY MEASUREMENTS

Spokespersons: G.A. Leksin, A.V. Vlassov [Moscow, ITEP]

E-mail contact leksin@vitep2.itep.ru, vlassov@vxitep.itep.ru

ITEP-896 (Taking data)

TWO-NEUTRINO DOUBLE BETA DECAY OF ${ }^{100}$ Mo TO THE FIRST EXCITED $0^{+}$STATE IN ${ }^{100} \mathrm{Ru}$

Spokespersons: A.S. Barabash [Moscow, ITEP], F.T. Avignone

[South Carolina U.]

E-mail contact barabash $\Theta$ vxitep.itep.ru

ITEP-897 (Completed data-taking 1991)

STUDX OF ${ }^{4}$ II $\pi^{-}$INTERACTIONS

Spokesperson: V.E. Grechko [Moscow, ITEP]

E-mail contact grechko@vitep5.itep.ru

ITEP-901 (Taking data)

QUASIELASTIC $\left(\pi^{-}, d\right)$ BACKWARD SCATTERING ON NUCLEI AT $0.7-1.3 \mathrm{GeV}$

Spokesperson: A.P. Krutenkova [Moscow, ITEP]

E-mail contact krutenkova@vxitep.itep.ru

ITEP-911 (Completed data-taking 1994)

FORWARD DIRECTION CUMULATIVE PARTICLE PRODUCTION AT $10 \mathrm{GeV}$

Spokespersons: Xu.T. Kiselev, V.A. Sheinkman [Moscow, ITEP]

E-mail contact yurikis@vxitep.itep.ru, yurikis@vxcern.cern.ch

ITEP-912 (Taking data)

SEARCH FOR NEUTRINOLESS DOUBLE BETA DECAY OF ${ }^{76} \mathrm{Ge}$

Spokespersons: I.V. Kirpichnikov [Moscow, ITEP], R.L. Brodzinski [Battelle Memorial Inst., Northwest], F.T. Avignone [South Carolina U.], A. Morales [Zaragoza U.]

E-mail contact kirpichnikov@vxitep.itep.ru,

amorales@gac.unizar.es
ITEP-914 (Taking data)

MEASUREMENT OF SPIN ROTATION PARAMETERS

$R$ AND $A$ IN ELASTIC $\pi p$ SCATTERING

By ITEP-PNPI Collaboration

Spokespersons: V.P. Kanavets [Moscow, ITEP], S.P. Kruglov [St. Petersburg, INP]

$\underline{E-m a i l ~ c o n t a c t}$ alekseev@vitep3.itep.ru, kruglov@Inpi.spb.su

ITEP-921 (Taking data)

INVESTIGATION OF QUASICOHERENT AND DEEP INELASTIC INTERACTIONS OF PIONS AND PRO-

TONS WITH NUCLEI

Spokespersons: V.S. Demidov, I.V. Kirpichnikov [Moscow, ITEP]

E-mail contact demidov@vxitep.itep.ru,

kirpichnikov@vxitep.itep.ru

ITEP-923 (Completed data-taking 1994)

INCLUSIVE PION DOUBLE CHARGE EXCHANGE ON LIGHT NUCLEI AT 0.7-1.3 GeV $/ c$

Spokesperson: A.P. Krutenkova [Moscow, ITEP]

E-mail contact krutenkova@vxitep.itep.ru,

kulikov@vxiteo.itep.ru

ITEP-941 ('laking data)

SUBTHRESHOLD $K^{+}$PRODUCTION IN PROTONNUCLEUS COLLISIONS

Spokespersons: Yu.T. Kiseiev, V.A. Sheinkman [Moscow, ITEP]

E-mail contact $\quad y$ urikis@vxitep.itep.ru, yurikis@vxcern.cern.ch

ITEP-942 (Taking data)

SEARCH FOR $D^{\prime}$ DIBARYON IN $p p$ INTERACTYONS

Spokesperson: A.I. Sutormin [Moscow, ITEP]

E-mail contact khanov@vitep5.itep.ru, kulikov@vxitep.itep.ru

ITEP-951 (Taking data)

SUBTHRESHOLD $K^{-}$PRODUCTION IN PROTONNUCLEUS COLLISIONS

Spokespersons: Yu.T. Kiselev, V.A. Sheinkman [Moscow, ITEP]

E-mail contact yurikis@vxitep.itep.ru, yurikis@vxcern.cern.ch

NEMO-2 (raking data)

DOUBLE BETA DECAY EXPERIMENTS WITH THE

TRACKING DETECTOR NEMO-2

By NEMO Collaboration

Spokesperson: S. Jullian [Orsay, LAL

E-mall contact jullian@lalcls.in 2 p $3 . f r$, barabash@vxitep.itep.ru

\section{ITEP Future Plans}

The ITEP's U-10 proton synchrotron will continue investigations of particle interactions with nuclei. 'The program includes experiments on the subthreshold production of particles, studies of cumulative effects, and a search for multi-quark states in nuclei. The synchrotron will also be used to continue polarization experiments in the resonance region, and in a search for exotic resonances. Test beams will be utilized in a development and testing of equipment for experiments at CERN, DESY, FNAL and other facilities. A special proton bcam will be devoted to the medical treatment of cancer patients. The study of double beta decays and other rare proccsses remains another important topic in the ITEP's experimental program. 


\section{SUMMARIES OF JINR (DUBNA) EXPERIMENTS}

\section{Selected JINR Experiments}

JINR-LHE-0941-1B 1993, In progress)

STUDIES OF POLARIZATION CHARACTERISTICS OF INELASTIC SCATTERING AND BREAKUP OF RELATIVISTIC POLARIZED DEUTERONS ON PROTONS AND COMPARISON WITH DATA OBTAINED WITH NUCLEAR TARGETS

DUBNA - L S Azhgirey, Y T Borzunov, E V Chernykh,

L B Golovanov, A D Kirillov, V P Ladygin, L V Malinina

P K Manyakov, N M Piskunov ( $\checkmark$ Spokesperson),

P A Rukoyatkin, I M Sitnik, G D Stoletov, E A Strokovsky

( $\checkmark$ Spokesperson), A L Svetov, A P Tsvinev, S A Zaporozhets

SAMARA AIRSPACE $U-P$ P Korovin

WILLIAM AND MARY COLL - C F Perdrisat

SOFIYA, INST CHEM TECH - $S$ Nedev

KIEV, I'TF - A P Kobushkin, A I Syamtomov

NORFOLK STATE U - V Punjabi

Accelerator JINR Detector ALPHA

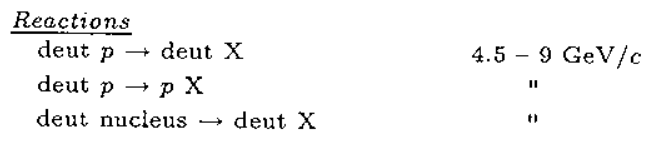

Particles studied $N\left(1440 P_{11}\right)$

Brief description One of the goals of the experiment is to study

the mechanism of the reaction $p\left(N, N^{\prime}\right) X$ with excitation of $\Delta$ and $N^{*}(1440)$ resonances. The $p\left(d, d^{\prime}\right) X$ reaction is chosen because the excitation of the Roper resonance is enhanced due to the isospin selection rules. A study of the expected interference effects between $\Delta$ and $N^{*}(1440)$ resonances in polarization observables of this reaction is planned. The data will provide valuable information about properties of the Roper resonance and also help resolve the existing theoretical uncertainties. It also measures the tensor analyzing power $T_{20}$ in inclusive deuteron breakup at $9 \mathrm{GeV} / c$ and $0^{\circ}$ on hydrogen and carbon targets.

Journal papers NIM A357 (1995) 386, and PL B361 (1995) 21.

Related experiments IINR-LHE-0941-1C, SALURNE-250, SATURNE-278

E-mail contact piskunov@sunhe.jinr.dubna.su, strok@sunhe.jinr.dubna.su

\section{Other JINR Experiments}

Listed here are some other Dubna experiments of interest to the particle physics community. Find more details about these projects online, in the SLAC's EXPERIMENT'S database (see p. 3), or contact the spokespersons. For a complete description of the current Lab's experimental research program, visit the JINR experiments' Web page at: http://sunhe.jinr.dubna.su/ strok/explist.html

\section{JINR-LHE-0936-3 (Taking data)}

SEARCH FOR NARROW HADRONIC RESONANCES PRODUCED IN NEUTRON-PROTON INTERACTIONS Spokesperson: Yu.A. Troyan [Dubna, JINR]

E-mail contact troyan@lhe09.jinr.dubna.su,

troyan@sunhe.jinr.dubna.su
JINR-LHE-0941-1A (Completed data-taking 1994)

MEASUREMENTS OF TENSOR ANALYSING POWER IN BACKWARD ELASTIC $d p$ SCATTERING AND BREAKUP AT $180^{\circ}$ (CM) BETWEEN $3.5-6.5 \mathrm{GeV} / c$ Spokespersons: N.M. Piskunov, I.M. Sitnik, E.A. Strokovsky [Dubna, JINR ]

E-mail contact piskunov@sunhe.jinr.dubna.su, sitnik@sunhe.jinr.dubna.su, strok@sunhe.jinr.dubna.su

\section{JINR-LHE-0941-1C (Taking data)}

SEARCH FOR $\triangle \triangle$ DIBARYON PRODUCTION USING SPIN DEPENDENT INELASTIC DEUTERONDEUTERON SCATTERING

Spokespersons: N.M. Piskunov (Dubna, JINR), F.W. Hersman [New Hampshire U.]

E-mail contact piskunov@sunhe.jinr.dubna.su, hersman@unh.edu

JINR-LHE-0941-1D (Completed data-taking Oct 1993)

MEASUREMENTS OF THE POLARIZATION TRANSFER COEFFICIENT IN BREAKUP OF RELATIVISTIC POLARIZED DEUTERONS INTO PROTONS EMITTED AT $0^{\circ}$

Spokespersons: N.M. Piskunov, I.M. Sitnik [Dubna, JINR] E-mail contact piskunov@sunhe.jinr.dubna.su, sitnik@sunhe.jinr.dubna.su

JINR-LHE-0941-1E (Completed data-taking Nov 1994)

MEASUREMENTS OF THE POLARIZATION TRANSFER COEFFICIENT AND TENSOR ANALYSING POWER IN BREAKUP OF RELATIVISTIC POLARIZED DEUTERONS INTO PROTONS EMITTED AT $0^{\circ}$ Spokespersons: L.N. Strunov, A.V. Zarubin [Dubna, JINR] E-mail contact strunov@sunhe.jinr.dubna.su

JINR-LHE-094I-3 (In preparation)

STUDIES OF SPIN-SPIN CORRELATIONS IN BACK WARD ELASTIC DEUTERON-PROTON SCATTERING Spokespersons: L.S. Azhgirey, I.M. Sitnik [Dubna, JINR], M.P. Rekalo [Kharkov, FTI]

E-mail contact sitnik@sunhe.jinr.dubna.su, azhgirey@cv.jinr.dubna.su

\section{JINR-LHE-0941-4 (Taking data)}

SPIN DEPENDENT TOTAL CROSS-SECTION DIFFERENCES IN $n p$ SCATTERING

Spokespersons: B.A. Khachaturov, N.M. Piskunov, V.X. Sharov, L.N. Strunov [Dubna, JINR], II.M. Spinka [Argonne], F. Lehar [Saclay]

E-mail contact khachaturov@main1.jinr.dubna.su, piskunov@sunhe.jinr.dubna.su, sharov@sunhe.jinr.dubna.su, strunov@sunhe.jinr.dubna.su. lehar@frcpn11.in2p3.fr, hms@hep.anl.gov

JINR-LHE-0941-5 (Completed data-taking 1992)

INVESTIGATION OF FSI AND INTERMEDIATE ISOBAR EFFECTS IN $4 \pi$ GEOMETRY $d p$ INTERACTIONS Spokespersons: V.V. Giagolev [Dubna, JINR], G. Martinska [Kosice U.], M.S. Nioradze [Tbilisi State U.], T. Siemiarczuk [Warsaw, INR]

E-mail contact glagolev@sunhe.jinr.dubna.su, martinov@kosicc.upjs.sk 
SUMMARIES OF JINR (DUBNA) EXPERIMENTS

JINR-LHE-0969 (Taking data)

INVESTIGATION OF PION-NUCLEON AND

NUCLEON-NUCLEON INTERACTIONS

Spokesperson: A.B. Kurepin [Moscow, INR]

E-mail contact kurepin@ai20.inr.troitsk.ru

JINR-LHE-0971-1 (Taking data)

MEASUREMENTS OF INCLUSIVE, QUASIELASTIC AND INELASTIC ANALYZING POWERS IN PROTONCARBON SCATTERING

Spokespersons: L.I. Sarycheva [Moscow State U.], A.I. Malakhov [Dubna, JINR]

E-mail contact lis@alex.npi.msu.su, malakhov@lhe32.jinr.dubna.su

JINR-LHE-0971-2 (In preparation)

MEASUREMENTS OF THE A DEPENDENCE OF THE LEADING PARTICLES YIELD FROM NUCLEI

Spokespersons: L.I. Sarycheva [Moscow State U.], A.I. Malakhov [Dubna, JINR]

E-mail contact lis@alex.npi.msu.su,

malakhov@lhe32.jinr.dubna.su

JINR-LHE-0983-4 (Taking data)

THE STUDY OF THE TENSOR ANALYZING POWER IN CUMULATIVE PARTICLES PRODUCTION ON A POLARIZED DEUTERON BEAM AT THE DUBNA SYNCHROPHASOTRON

Spokespersons: A. Litvinenko, L. Zolin [Dubna, JINR]

E-mail contact litvin@moonhe.jinr.dubna.su

JINR-LHE-1010 (In preparation)

INVESTIGATION OF SPIN PHENOMENA IN THE ENERGY RANGE CORRESPONDING TO TRANSITION FROM NUCLEON TO QUARK-GLUON DEGREES OF FREEDOM IN NUCLEY

Spokespersons: A.A. Baldin, A.S. Vodopianov [Dubna, JINR]

E-mail contact baldin@sunhe.jinr.dubna.su, vodopian@sunhe.jinr.dubna.su

JINR-LHE-1011-1 ('Taking data)

MEASUREMENTS OF INCLUSIVE AND CORRELATION CHARACTERISTICS IN CUMULATIVE KINEMATICAL REGION IN $d p$ AND $d d$ INTERACTION WITH POLARIZED DEUTERON BEAM

Spokespersons: Yu.A. Panebratsev, S.S. Shimanskiy [Dubna, JiNR]

E-mail contact panebrat@sunhe.jinr.dubna.su, shimansk@sunhe.jinr.dubna.su

JINR-LNP-09 (Taking data)

SEARCH FOR NARROW DIBARYON RESO-

NANCES IN THE DOUBLE PROTON-PROTON RREMSSTRAHLUNG REACTION AT THE ENERGY BELOW THE PION THRESHOLD

Spokesperson: A.S. Khrykin [Dubna, JINR]

E-mail contact khrykin@nusun.jinr.dubra.su
JINR-LNP-23 (Taking data)

THE EXPERIMENTAL STUDY OF $n p$ ELASTIC SCAT TERING AMPLITUDES AT $16 \mathrm{MeV}$

Spokespersons: Yu.A. Usov [Dubna, JINR], I. Wilhelm [Charles

U.]

E-mail contact usov@main1.jinr.dubna.su, ivan.wilhelm@ruk.cuni.cr

\section{JINR Future Plans}

The 1996-98 JINR Scientific Program shows a clear commitment to continuation of both the theoretical (particularly related to phenomenology), and the experimental research. The experimental groups will be working at Dubna and at other institutions (IHEP, CERN, DESY, BNL, ...). The at-home experiments include (i) the studies of polarization phenomena using beams of polarized deuterons and nucleons from the LHE acceleration complex, and (ii) investigations of the color degrees of freedom in the nuclear matter with the Nuclotron relativistic nuclei beams, at the transition energies region where the non-perturbative QCD effects are considered significant. The priorities in the machine physics are the completion of the slow extraction system for the Nuclotron, and further development of the extracted beam infrastructure at the LIIE complex. [Nuclotron is a new superconductive synchrotron, built for acceleration of protons up to the kinetic energy of $12.8 \mathrm{GeV}$, and deuterons and other nuclei, including uranium, up to $6 \mathrm{GcV} / \mathrm{A}$ (for $\mathrm{Z} / \mathrm{A}=1 / 2$ )]. 


\section{SUMMARIES OF KEK EXPERIMENTS}

\section{Selected KEK Experiments}

\section{KEK-TE-001}

(Approved Mar 1983, Began data-taking Nov 1986, Completed data-taking May 1995)

TRISTAN $e^{+} e^{-}$EXPERIMENTS BY THE VENUS COLLABORATION

VENUS COLLABORATION

TOKYO METROPOLITAN U - M Chiba, T Hirose, N Hosoda, T Oyama, F Yabuki

TOHOKU U - K Abe, J MacNaughton

KEK - K Amako, Y Arai, M Fukawa, Y Fukushima, F Hinode,

N Ishihara, N Kanematsu, J Kanzaki, T Kondo, T Matsui

(Spokesperson), S Odaka, K Ogawa, T Ohama, M Sakuda,

N Sato, I Shirai, $\Upsilon$ Sumiyoshi, F Takasaki, $\Upsilon$ Tsuboyama,

$S$ Uehara, Y Unno, M Utsumi, Y Watase, Y Yamada

'TSUKUBA U, INST APPL PHYS - T Arima, Y Asano

(Spokesperson), II Hamasaki, M Miura, S Mori, M Shirakata, Y Takada, K Yusa

YASUDA WOMENS JR COLL - Y Chiba

WAKAYAMA MEDICAL COLL - M Daigo

OSAKA U - J Haba, H Hanai, N Kanda, A Kruger, Y Nagashima, A Suzuki, H Takaki, M Takita, D Tatsumi, Y Yamamoto

KYOTO U - Y Hemmi, R Kikuchi, H Kurashige, K Miyake,

A Okamoto, H Sakamoto

TOHOKU GAKUIN U - M Higuchi, Y Hoshi, M Sato

KOBE U - Y Homma, A Ono

HIROSHIMA U .. Y Iwata, T Ohsugi, H Ohyama

KEK \& HELSINKI U - T T Korhonen

OKAYAMA U - E K Matsuda, K Okabe, N Tamura

TOKYO, INTERNATIONAL CHRISTIAN $U$ - Y Nakagawa,

$T$ Yamagata

MIYAZAKI U - T Nakamura

TSUKUBA U - I Nakano

IBARAKI COLL TECH . M Shioden

KOGAKUIN U - K Tobimatsu, T Watanabe

TSUKUBA COLL TECH - Y Yonezawa

NARUTO U OF EDUCA'TYON - H Yoshida

Accelerator KEK-TRISTAN Detector VENUS

Reactions

$$
e^{+} e^{-} \quad<70 \mathrm{GeV}\left(\mathrm{E}_{\mathrm{cm}}\right)
$$

Brief description Physics topics include a reevaluation of the clectroweak processes with higher precision and studies of $\mathrm{QCD}$ through quark-pair production in photon-photon collisions. The integrated luminosity for the experiment reached 400 inverse picobarns. Data analysis in progress (June 96).

Journal papers NIM 217 (1983) 181, JJAP 23 (1984) 897, NIM A228 (1985) 309, NIM A238 (1985) 328, NIM A243 (1986) 58 NIM A253 (1986) 27, IEEE TNS 33 (1986) 73, JJAP 25 (1986) 1049, NIM A254 (1987) 35, NIM A254 (1987) 317, NIM A259 (1987) 430, NIM A259 (1987) 438, JJAP 26 (1987) 982, JPSJ 56 (1987) 3763, JPSJ 56 (1987) 3767, PL B198 (1987) 570, PRL 59 (1987) 2915, NIM A265 (1988) 457, NIM A269 (1988) 171, NIM A269 (1988) 522, NIM A270 (1988) 319, NIM A271 (1988) 432, NIM A272 (1988) 687, IEEE TNS 35 (1988) 300, PL B207 (1988) 355, PL B213 (1988) 400, PRL 61 (1988) 915, NIM A274 (1989) 183, NIM $\Lambda 281$ (1989) 462, IEEE TNS 36 (1989) 665, IEEE TNS 36 (1989) 670, JJAP 28 (1989) 1981, JPSJ 58 (1989) 3037, PL B232 (1989) 425, PL B232 (1989) 431, PRL 63 (1989) 1776, ZPHY C45 (1989) 175, PR D39 (1989) 3524, PL B234 (1990) 202, PL B234 (1990) 382, PL B240 (1990) 232, PL B246 (1990) 297, ZPHY C48 (1990) 13, NIM A301 (1991) 497, NIM A303 (1991) 346, NIM A305 (1991) 71, PL B264 (1991) 212, PI B266 (1991) 188, PL B267 (1991) 309, PRL 66 (1991) 280. NIM A311 (1992) 57, NIM A322 (1992) 211, NIM A323 (1992) 471, PL B278 (1992) 393, PL B278 (1992) 499, NIM A330 (1993) 64. PL B302 (1993) 119, PL B313 (1993) 245, PL B313 (1993) 288, PRL 71 (1993) 38, NMM A340 (1994) 501, PL B331 (1994) 211, PRL 72 (1994) 3313, ZPHY C63 (1994) 213, JPSJ 64 (1995) 435 , and ZPHY C69 (1996) 597.

E-mail contoct takayuki.matsui@kek.jp WWW Home-page http://venusux1.kek.jp/
KEK-TE-002

(Approved Mar 1983, Began data-taking Nov 1986, Completed data-taking May 1995)

\section{STUDY OF $e^{+} e^{-}$ANNIHILATION PHENOMENA BY A DETECTOR WITH PARTICLE IDENTIFICATION}

\section{TOPAZ COLLABORATION}

NARA WOMENS U - K Adachi, S Awa, N Fujiwara, H Klayashii, H Ikeda, M Iwasaki, Y Kayahara, K Miyabayashi, K Muramatsu, T Nagira, M Nakajima, H Nishioka, N Nishiwaki, S Noguchi, M Ohkura, E Sakai, M Takemoto, N Toomi, A Yamaguchi, Y Yoake

TOKYO U OF AGRIC TECH - K Emi, N Fujio, K Harigae,

If Hirano, S Hori, K Iwashiro, H Mamada, O Nitoh, S Onodera, T Shinohara, K Takahashi, O Tsumura, J Yoshizawa

NAGOYA U .. K Abc, T Abe, M Aoki, R Kajikawa

(Spokesperson), K Kurata, II Masuda, K Matsushita

K Nakabayashi, N Ohishi, T Ohshima, N Sasayama,

K Shimozawa, A Sugiyama, S Suzuki, H Takamure, F Teramae, M Tomoto, T Toyama

KEK - I Adachi, R Belušević, H B Dijkstra, M Doser, R Enomoto, H Fujii, K Fujii, J Fujimoto, N Iida, H Ikeda, R Itob,

H Iwasaki, S Iwata, S Kawabata (Spokesperson), H Kichimi, M Kobayashi, S Kuroda, T Matsuda, A Miyamoto, K Nakamura Y Ohnishi, H Ozaki, T Sato, R Sugahara, $T$ Suwada, $T$ 'Tauchi,

I'I'sukamoto, S Uno, O Yamakawa, A Yamamoto, M Yamauchi, $Y$ Yoshimura, $M$ Yoshioka

TOKYO U - H Aihara, T Kamae, T Kishida, N Kusuki, F Sai, A Shirahashi, S Yamamoto, S Yamashita

OSAKA CI'TY U - K Fujita, S Iigashi, Y Inoue, Y Kato,

A Maruyama, M Nakamura, E Nakano, M Nakayama,

T Okusawa, T Sato, A Shimonaka, T Takahashi, T Tanaka $Y$ Teramoto

PURDUE U - B Howell, D Kollick, I Levinc, E Shibata

TOKYO INS'T 'TECH -- M Aoki, K Kaneyuki, S Minami,

N Nakagawa, A Ochi, Y Ohshima, N Sugiyama, T Tachibana,

$T$ Tanimori, K Watanabe, $Y$ Watanabe

TOKYO U, INS -- A Imanishi, T Ishii, S Kato, K Maruyama, T Morimoto, H Okuno

KOBE U - T Fujii, K Fujiwara, K Nagai

TEZUKAYAMA U - F Ochiai

Accelerator KEK-TRISTAN Detector TOPAZ

Reactions

$$
e^{+} e^{-} \quad<70 \mathrm{GeV}\left(\mathrm{E}_{\mathrm{cm}}\right)
$$

Brief description Searches for new particles such as heavy quarks, heavy leptons, and various supersymmetric particles, and studies in detail electroweak as well as QCD phenomena. The detector has large solid angle coverage with very good particle identification and 3-dimensional tracking capabilities. The total integrated luminosity for the experiment is about 330 inverse picobarns. Data analysis in progress (June 96).

Journal papers NIM A225 (1984) 23, NIM A236 (1985) 55, NIM A252 (1986) 423, NIM A256 (1987) 449, NIM A269 (1988) 507. NIM A269 (1988) 513, NIM A270 (1988) 11, NIM A271 (1988) 404, PL B200 (1988) 391, PL B208 (1988) 319, PRL 60 (1988) 97, PR D37 (1988) 1339, PL B218 (1989) 105, PL B227 (1989) 495, PL B228 (1989) 553, PL B229 (1989) 427. NIM A297 (1990) 148, PL B234 (1990) 185, PL B234 (1990) 197, PL B234 (1990) 525, PL B240 (1990) 513, PL B244 (1990) 352, PL B249 (1990) 336, NIM A300 (1991) 575, PL B255 (1991) 613, PL B268 (1991) 457, NIM A312 (1992) 440, NIM A316 (1992) 202, PL B278 (1992) 506, PL B279 (1992) 422, PL B284 (1992) 144, PL B291 (1992) 206, NIM A334 (1993) 367, PL B304 (1993) 373, PL B313 (1993) 475, PL B314 (1993) 149, PL B314 (1993) 471, PL B328 (1994) 535, PL B332 (1994) 477, PL B340 (1994) 135, PL B341 (1994) 99, PL B341 (1994) 238, PR D50 (1994) 1879, PL B345 (1995) 335, PL B347 (1995) 171, PL B347 (1995) 179, PL B361 (1995) 199, and PL B368 (1996) 299.

E-mail contact kajikawa@kekvax.kek.jp

WWW Home-page http://tophpl.kek.jp/ 


\section{SUMMARIES OF KEK EXPERIMENTS}

\section{KEK-TE-003}

(Proposed 1983, Approved Nov 1983, Began data-taking Nov 1986, Completed data-taking Jun 1994)

\section{AMY - A HIGH RESOLUTION LEPTON DETECTOR FOR TRISTAN}

\section{AMY COLLABORATION}

ROCHESTER U - A Bodek, B J Kim, T Kumita, Y K Li,

$C$ Velissaris

SOUTH CAROLINA U - C Rosenfeld, S Wilson

KOREA U - J S Kang, D Y Kim

LOUISIANA STATE U - P Kirk

BEIJING, IHEP - M H Ye, Z P Zheng

VIRGINIA TECH - A Abashian, K Gotow, D Haim,

M E Mattson, L Piilonen

UC, DAVIS - $\mathrm{R}$ E Breedon, W Ko, $\mathrm{R} L$ Lander, J Rowe, J $R$ Smith, D Stuart

HAWAII U - S Kanda, S L Olsen (Spokesperson), K Ueno

KEK - K Abe (Spokesperson), Y Fujii, Y Kurihara, F Liu,

A Maki, $T$ Nozaki, $T$ Omori, H Sagawa, Y Sakai, T Sasaki,

Y Sugimoto, Y Takaiwa, S Terada

GYEONGSANG NATIONAL U - S K Choi

KONAN U - F Kajino

MINNESO'TA U - T l'homas

NIIGATA $U$ - $T$ Aso, K Miyano, H Miyata, $N$ Takashimizu

NIHON DEN'IAL COLL - Y Yamashita

RUTGERS U - F Sannes, S Schnetzer, R Stone, J Vinson

SAGA U, JAPAN - S Behari, S Kobayashi, A Murakami,

K S Saroj

SEOUL NATIONAL U - S K Kim, M H Lee, S S Myung

KYUNGPOOK NATIONAL U - D Son

CHUO U, TOKYO - S Matsumoto

SAITAMA U -- T Ishizuka

Accelerator KEK-TRISTAN Detector AMY

Reactions

$$
e^{+} e^{-} \quad<70 \mathrm{GeV}\left(\mathrm{E}_{\mathrm{cm}}\right)
$$

Brief description Data analysis in progress (June 96).

Journal papers IEEE TNS 23 (1987) 520, NIM A260 (1987) 361, NIM A265 (1988) 141, PRL 60 (1988) 93, PRL 60 (1988) 2359, PRL 61 (1988) 911, NIM A274 (1989) 95, NIM A283 (1989) 665, PL B218 (1989) 112, PL B218 (1989) 499, PL B223 (1989) 476, PL B228 (1989) 548, PRL 62 (1989) 1713, PRL 63 (1989) 1342 PRL 63 (1989) 1772, PRL 63 (1989) 1910, PRL 63 (1989) 2341, PL B234 (1990) 534, PL B240 (1990) 243, PL B244 (1990) 573 PL B252 (1990) 491, PRL 64 (1990) 984, PR D41 (1990) 2675, PR D42 (1990) 737, PR D42 (1990) 949, PR D42 (1990) 1339, IJMP A6 (1991) 2583, NIM A307 (1991) 52, NIM A.317 (1992) 75, NIM A323 (1992) 601, PL B277 (1992) 215, PL B303 (1993) 385, PL B313 (1993) 469, PL B325 (1994) 248, and PL B346 (1995) 208

E-mail contact abez@kekvax.kek.jp, solsen@uhhepb.phys.hawaii.edu

WWW Home-page http://amywww.kek.jp/

\section{KEK-162}

(Proposed 1987, Approved Oct 1987, Began data-taking 1996, In progress)

MEASUREMENT OF THE $C P$-VIOLATING DIRECT AMPLITUDE IN $K_{L}^{0} \rightarrow \pi^{0} e^{+} e^{-}$DECAY

KYOTO U - H Kurashige, T T Nakamura, T Nomura,

H Sakamoto, N Sasao (Spokesperson), M Suehiro

KEK - Y Eukushima, M Noumachi, O Sasaki, T Taniguchi

Accelerator KEK-PS Detector TOKIWA

Reactions

$$
K_{L} \rightarrow \pi^{0} e^{+} e^{-}
$$

Particles studied $K_{L}$

Brief description The apparatus consists of large drift chambers,

a UV-sensitive Cerenkov counter for detection of electrons,

and an electromagnetic CsI calorimeter with a good energy resolution. The drift chambers use argon and $\mathrm{CF}_{4}$ gas. Sensitive to branching ratios smaller than $10^{-10}$. Taking data (July 96).

Journal papers NIM A270 (1988) 106, NIM A283 (1989) 709, and NIM A317 (1992) 213.

E-mail contact sasao@kekvax.kek.jp

\section{KEK-231}

(Proposed 1990, Approved Jul 1990, Began data-taking 1994, Completed data-taking 1994)

STUDY OF VIOLATION OF TIME REVERSAL INVARIANCE IN NEUTRON REACTIONS

KEK - T Adachi, S Ishimoto, Y Masuda (Spokesperson), Y Mori, K Morimoto, H M Shimizu

KYOTO U - M Iinuma, A Masaike, Y Matsuda

TOKYO INST TECH - K Asahi, M Harada, H Sato

TOHOKU U - K Sakai, S Tanaka, A Yamaguchi

Accelerator KEK-PS Detector Counter

Reactions Polarized beam and target

$$
{ }^{139} \mathrm{La} \rightarrow n \mathrm{X}
$$$$
n^{81} \mathrm{Br} \rightarrow n \mathrm{X}
$$

Journal papers NIM A264 (1987) 169, NP A504 (1989) 269, and HFI 74 (1992) 149

E-mail contact masuda@kekvax.kek.jp

\section{KEK-246}

(Approved Jul 1991, In progress)

SEARCH FOR T-VIOLATING MUON POLARIZATION IN $K^{+} \rightarrow \pi^{0} \mu^{+} \nu$ DECAY USING STOPPED KAONS

E246 COLLABORATION

KEK - J Imazato (Spokesperson), Y Kuno, H M Shimizu, K H Tanaka

TOKYO U, INS *. M Aoki, Y Fujita, H Outa, S Sugimoto,

$T$ Yamazaki

TOKYO U - R S Hayano, ' I Ishikawa, H Tamura

MOSCOW, INR - D V Dementyev, M Grigorev, A P Ivashkin,

M M Khabibullin, Y G Kudenko, V M Lobashev, O V Mineev,

$\checkmark$ Popov

ISUKUBA U - I Arai, $X$ Igarashi, ' $I$ Ikeda, $M$ Ise, $K$ Shibata

TSUKUBA U, INST APPL PHYS .. M Abe, $Y$ Asano

IBARAKI U, HITACHI - T Yokoi

TOKYO INST TECH - S Shimizu

SASKATCHEWAN U - T Baker, C Rangacharyulu, Y M B Shin

YONSEI U - E J Kim, J M Lee, $X \mathrm{H}$ Shin

KYUNGSUNG U - Y M Park

BRITISH COLUMBIA U - P Gumplinger, M Hasinoff, E Saettler

TRIUMF - J Doornbos, R Fenderson, IA Macdonald,

$N$ Stevenson

MONTREAL U - P Depommier

VIRGINIA TECH - M Blecher

KANAGAWA U - A Kaga

Accelerator KEK-PS Detector Spectrometer

Reactions

$$
K^{+} \rightarrow \pi^{0} \mu^{+} \nu \quad 0 \mathrm{GeV} / c\left(\mathrm{P}_{1 \mathrm{lab}}\right)
$$

Particles studied $K^{+}$

Brief description Uses the Superconducting Toroidal Spectrome-

ter. Approved for 450 shifts. Taking data (July 96).

E-mail contact imazato@kekvax.kek.jp, kekvax:imazato 


\section{SUMMARIES OF KEK EXPERIMENTS}

\section{KEK-304}

(Approved 1995, Began data-taking 1996, In progress)

STUDY OF $K^{+} \rightarrow \pi^{0} e^{+} \nu$

Accelerator KEK-PS Detector ?

Particles studied $K^{+}$

Brief description A collaboration of KEK, Tokyo Inst. Tech. and Tokyo U. Taking data (July 96). For further information, please contact the Spokesperson, Dr. Takayoshi Ohshima [KEK]. E-mail contact ohshima@kekvax.kek.jp

\section{KEK-362}

(Approved 1995, In preparation)

A LONG BASELINE NEUTRINO OSCILLATION EXPERIMENT USING KEK-PS AND SUPER KAMIOKANDE

\section{Accelerator KEK-PS Detector ?}

Particles studied $\nu$

Brief description An international collaboration of scientists from Japan and USA. In preparation (July 96). For further information, please contact the Spokesperson, Dr. Koichiro Nishikawa [KEK]

E-mail contact nishikaw@kekvax.kek.jp

WWW Home-page http://pnahp.kek.jp/

\section{KEK-BF-BELLE}

(Proposed 1994, Approved Mar 1994, In preparation)

\section{A STUDY OF $C P$ VIOLATION IN $B$ MESON DECAYS} BELLE COLLABORATION

Accelerator KEK-B-FACTORY Detector BELLE

Brief description KEK $B$-factory is expected to be capable of producing more than ten million $B$ meson pairs each year.

When the facility becomes operational, the proposed experiment

will study various aspects of $B$ meson physics, including the long-standing puzzle of the violation of $C P$ symmetry. The collaboration consists of physicists from six countries and more

than 36 institutions. 'The letter of Intent has been accepted in March 94. Expected to begin data taking in FY 1998/99.

For more information, please contact the Spokespersons,

Dr. Shiro Suzuki [Nagoya U.], Dr. Stephen L. Olsen [Hawaii U.\},

or Dr. Fumihiko Takasaki [KEK].

E-mail contact suzuki@hepl.phys.nagoya-u.ac.jp,

solsen@uhhepg.phys.hawaii.cdu, fumihiko@kekvax.kek.jp

WWW Home-page http://bsunsrvi.kek.jp/

\section{Other KEK Experiments}

Listed here are some other KEK experiments of interest to the particle physics community. Find more detajls about these projects online, in the SLAC's EXPERIMENTS database (see p. 3), or contact the spokespersons. Check also the KEK's yearly publication 'Annual Report' for a complete list of active experiments.

KEK-140A (Completed data-taking Mar 1993)

STUDY OF HEAVY HYPERNUCLEI VIA THE $(\pi, K)$ REACTION USING THE SKS DETECTOR

Spokesperson: O. Hashimoto ['Tokyo U., INS]

E-mail contact hashimot@ins.u-tokyo.ac.jp
KEK-218 (Completed data-taking Feb 1993)

STUDY OF THE FORMATION OF ${ }_{\Lambda}^{4} \mathrm{H}$ BY USING $\pi \pi$ COINCIDENCE

Spokesperson: H. Tamura [Tokyo U.]

E-mail contact tamura@tkyvax.phys.s.u-tokyo.ac.jp, tkyvax: tamura

KEK-224 (Completed data-taking Jan 1992)

SEARCH FOR THE $H$-DIBARYON WITH A SCINTILLATING FIBER TRACK DETECTOR

Spokesperson: K. Imai [Kyoto U.]

E-mail contact imai@kekvax.kek.jp, kytvax::imai

K.EK-228 (Completed data-taking 1995)

PRECISE MEASUREMENT OF THE STRONG INTERACTION SHIFTS OF KAONIC HYDROGEN $X$-RAYS

Spokesperson: M. Iwasaki [Tokyo U., INS]

KEK-235 (Completed data-taking Apr 1993)

DIFFERENTIAL CROSS-SECTION FOR $p(n, \gamma) d$

Spokesperson: M.A. Kovash [Kentucky U.]

E-mail contact kovash@ie.pa.uky.edu

KEK-248 (Taking data)

SEARCH FOR $H$ PARTICLES IN THE $p p \rightarrow K^{+} K^{+}$ REACTION

Spokesperson: H. Kawai [Chiba U.]

KEK-251 (Completed data-taking Dec 1992)

HYPERON-NUCLEON SCATTERING EXPERIMENT I $\Sigma^{+} p$ SCATTERING

Spokesperson: M. Ieiri [KEK, Tsukuba]

E-mail contact ieiri@kekpsa.kek.jp

KEK-257 (Completed data-taking May 1992)

SUBTHRESHOLD ANTIPROTON PRODUCTION IN

d A REACTIONS

Spokesperson: J. Chiba [KEK, Tsukuba]

E-mail contact chibaj@kekvax.kek.jp

KEK-262 (Completed data-taking Nov 1993)

BEHAVIOR OF PIONIC HYDROGEN ATOMS. PART II

Spokesperson: A. Shinohara [Nagoya U.]

KEK-269 (Completed data-taking 1995)

DLFFERENTYAL $\pi$ C CROSS-SECTION ABOVE THE RESONANCE REGION

Spokesperson: Harutaka Sakaguchi [Kyoto U.].

E-mail contact sakaguchi@kytvax.scphys.kyoto-u.ac.jp

KEK-278 (Completed data-taking Dec 1993)

ASYMMETRY OF NON-MESONIC WEAK DECAY OF POLARIZED ${ }_{\Lambda}^{5} \mathrm{He}$

Spokesperson: Tadafumi Kishimoto [Osaka U.;

E-mail contact kisimoto@phys.sci.osaka-u.ac.jp 
SUMMARIES OF KEK EXPERIMENTS

KEK-287 (Completed data-taking Jul 1993)
STUDY OF MN SPIN-SPIN INTERACTION USI
SPECTROSCOPY OF HYPERFRAGMENTS
Spokesperson: Hiro Tamura [Tokyo U.]
E-mail contact tamura@tkyvax.phys.s.li-tokyo.ac.jp,
tkyvax::tamura

KEK-289 (Taking data)

HYPRON-NUCLEON SCATTERING EXPERIMENT:

$\Sigma^{-} p$ AND $\Lambda p$ SCATTERING

Spokesperson: Masaharu Ieiri [KEK, Tsukuba]

$\underline{E-m a i l ~ c o n t a c t}$ iciri@kekpsb.kek.jp

KEK-307 (Completed data-taking 1995)

LIFETIMES AND WEAK DECAY WIDTHS OF LIGHT

AND MEDIUM HEAVY $\Lambda$ HYPERNUCLEI

Spokesperson: H.C. Bhang [Scoul National U.]

E-mail contact bhang@kekvax.kek.jp

KEK-325 (Taking data)

NUCLEAR MATTER EFFECT ON MESON MASS IN $\phi$ DECAY

Spokesperson: Hideto Enyo [Kyoto U.]

E-mail contact enyo@kekvax.kek.jp,

enyo@pn.scphys.kyoto-u.ac.jp

KEK-326 (Completed data-taking 1994)

STUDY OF HYPERFRAGMENT PRODUCTION IN

ABSORPTION OF STOPPED $K^{-}$

Spokesperson: H. Tamura (Tokyo U.)

E-mail contact tamura@tkyvax.phys.s.u-tokyo.ac.jp,

tkyvax::tamura

KEK-336 (Taking data)

SPECTROSCOPIC INVESTIGATION OF LIGHT $\Lambda$

HYPERNUCLEI BY THE $\left(\pi^{+}, K^{+}\right)$REACTION

Spokesperson: O. Hashimoto [Kyoto U.]

E-mail contact hashimot@ins.u-tokyo.ac.jp

KEK-352 (Taking data)

QUASIELASTIC $\pi$ SCATTERING

Spokesperson: Roy J. Peterson [Colorado U.]

E-mail contact peterson@spectr.colorado.edu

\section{KEK Future Plans}

The interdisciplinary research with the $12-\mathrm{GeV}$ Proton Syn-

chrotron will continue utilizing the proton and light ion beams.

'lhe particle physics studies will focus on the long baseline neu-

trino oscillation experiment with the SupcrKamiokande detector,

and the time-reversal invariance experiment on the kaon system.

Both experiments will need twice (or more) intenser proton beams

than presently available, and an accelerator study group is being

formed to deal with the new requirements. 'l'he TRISTAN collider

has been closed, and its tunnel is now used for two rings of the

KEK-B ( $B$ Factory) asymmetric electron-positron collider $(8 \times 3.5$

GeV). The machine is scheduled to be commissioned in FY 1998-

99 , and the design luminosity is $10^{34} \mathrm{~cm}^{-2} \mathrm{~s}^{-1}$. 


\section{SUMMARIES OF LOS ALAMOS EXPERIMENTS}

\section{Selected LAMPF Experiments}

\section{LAMPF-969}

(Proposed Jul 1985, Approved Aug 1985, Began data-taking Jun 1992, Completed data-taking 1995)

MEGA - SEARCH FOR THE RARE DECAY $\mu^{+} \rightarrow e^{+} \gamma$ MEGA COLLABORATION

UCLA - D Barlow, B M K Nefkens, B Tippens

CHICAGO U - J Crocker, S C Wright

FERMILAB - P S Cooper

HAMPTON U - L Tang

HOUSTON U - M Barakat, Y Chen, M Dzemidzic, A Empl,

J Flick, E V Hungerford, K Johnston, K Lan, B W Mayes,

$R$ Phelps, L Pinsky, $W$ von Witsch

INDIANA U - J Knott, K M Stantz, J Szymanski

LOS ALAMOS - J F Amann, K Black, $R$ D Bolton, M Brooks,

$S$ Carius, M D Cooper ( $\checkmark$ Spokesperson), W Foreman,

C M Hoffman, G E Hogan, T Kozlowski, M Kroupa, D Lee,

G Mills, R E Mischke, F J Naivar, M A Oothoudt, C Pillai,

R D Werbeck, D Whitehouse, C Wilkinson

QUEENS U, KINGSTON - A Fallin

STANFORD U - E B Hughes, C Jui, I N Otis, M W Ritter

TEXAS A AND $M-C$ Gagliardi, G Kim, F Liu, $R$ E Tribble,

$\mathrm{X} T \mathrm{~T}, \mathrm{~L}$ Van Ausdeln, $\mathrm{X}$ Zhou

VALPARAISO U, INDIANA - R Fisk, D D Koetke,

R W Manweiler, $S$ Stanislaus

ViRGINIA U - R Marshall, B Wright, $\mathrm{K}$ O H Ziock

VIRGINIA TECH - D Haim, F Lee, L E Piilonen, Y Zbang,

W Zhou

WYOMING U - A R Kunselman

YALE U - K Hahn, J Markey

Accelerator LAMPF Detector MEGA

Reactions Polarized beam

$$
\mu^{+} \rightarrow e^{+} \gamma \quad 0 \mathrm{MeV} / \mathrm{c}\left(\mathrm{P}_{\mathrm{lab}}\right)
$$

Particles studied $\mu^{+}$

Brief description Also searches for a $V+A$ contribution to radiative decay. Approved for 4000 hours. Looks for $\mu^{+} \rightarrow e^{+} y$ at a level of $6 \times 10^{-13}$, a factor of 80 better than the Crystal Box detector. Took data in $1992 / 93$ and in 1994/95. Data analysis in progress (July 96)

Journal papers NIM A303 (1991) 298, NIM A323 (1992) 198,

NIM A349 (1994) 1I8, and NIM A372 (1996) 195

Related experiments PSI-R-87-03

E-mail contact mcooper@lanl.gov

\section{LAMPF-1054}

(Proposed Dec 1986. Approved Feb 1987, Began data-taking 1991, Completed data-taking 1996)

ULTRAHIGH PRECISION MEASUREMENTS ON THE MUONIUM GROUND STATE: HYPERFINE STRUCTURE AND MUON MAGNETIC MOMENT

LOS ALAMOS - C Pillai, O van Dyck

HEIDELBERG U, PHYS INST - K Jungmann, I Reinhard,

G zu Putlitz ( $\checkmark$ Spokesperson)

YALE U * M G Boshier, S Dhawan, X Fei, $V W$ Hughes

( $\checkmark$ Spokesperson), D Kawall, W Liv

YALE U \& PSI, VILLIGEN - M Janousch

BROOKHAVEN - F Mariam, $K$ Woodle

LIVERMORE - P Egan

Accelerator LAMPF Detector Other

Particles studied muonium

Brief description An ultrahigh precision measurement of the muonium hyperfine structure interval $\Delta \nu$ and of the microwave magnetic moment ratio $\mu_{\mu} / \mu_{p}$ with the goal of determining $\Delta \nu$ to $10 \mathrm{ppb}$ and $\mu_{\mu} / \mu_{p}$ to $60 \mathrm{ppb}$. Uses the microwave magnetic resonance spectroscopy method with an intense and pure subsurface $\mu^{+}$beam, a large superconducting homogeneous solenoid, and a line-narrowing method involving a chopped $\mu^{+}$ beam. Data analysis in progress (July 96).

Journal papers NIM A333 (1993) 260, and PR A52 (1995) 1948.

Related experiments BNL-821

E-mail contact vernon.hughes@yale.edu

\section{LAMPF-1173 (LSND)}

(Proposed Jul 1989, Approved Jan 1990, Began data-taking Sep 1993 , In progress)

SEARCH FOR $\bar{\nu}_{\mu} \leftrightarrow \bar{\nu}_{e}$ OSCILLATIONS WITH HIGH SENSITIVITY

LSND COLLABORATION

UC, RIVERSIDE - R M Gunasingha, $\mathrm{K}$ Mcllhany, I Stancu,

W Strossman, G I VanDalen

UC, SAN DIEGO - W Vernon

UC, SANTA BARBARA - D O Caldwell, M Gray, S Yellin

EMBRY-RIDDLE AERONAUTICAI U - D Smith, I Walt

UCIIRPA, SLAC - A Eisner, Y Wang

LINFIELD COLL, OREGON .. I Cohen

LOS ALAMOS - R Burman, J Donahue, $F$ J Federsoiel,

G ' $\mathrm{I}$ Garvey, W C Louis ( $\sqrt{ }$ Spokesperson), G B Mills,

$V$ Sandberg, $R$ Tayloe, $D$ H White

LOUISIANA STATE U - R Imlay, H J Kim, W Metcalf, N Wadia

LOUISIANA TECH U - $K$ Johnston

NEW MEXICO U - B B Dieterle, R Reeder

SOUTHERN U - A Fazely

TEMPLE U - C Athanassopoulos, L B Auerbach, R Majkic.

J Margulies, D Works, Y Xiao

Accelerator LAMPF Detector LSND

Reactions

$\bar{\nu}_{\mu} \rightarrow \bar{\nu}_{e}$

$\nu_{\mu} \rightarrow \nu_{e}$

$\nu_{e} \mathrm{C} \rightarrow e^{-}$nucleon

$\nu_{\mu} \mathrm{C} \rightarrow \mu^{-}$nuclcon

$\nu \mathrm{C} \rightarrow \nu \mathrm{C}^{*}$

$\nu_{\mu} p \rightarrow \nu_{\mu} p$

$\nu_{c} e^{-} \rightarrow \nu_{c} e^{-}$

Particles studied $\nu$

Brief description A search for neutrino oscillations to the level

$\sin ^{2} \theta=3 \times 10^{-4}$, where $\theta$ represents the mixing angle if thero were a two-generation mixing. Uses neutrinos produced by both at-rest and in-flight decaying pions. Neutrinos then interact in a mineral oil $\left(\mathrm{CH}_{2}\right)$ target. The detector consists of a tank with 167 tons of liquid scintillator and with 1220 photomultiplier tubes mounted on the inside tank surface. Other physics goals include measurements of neutrino elastic, charged-current, and neutral-current scattering. Taking data (July 96). Will continuc the data taking for at least one more year, and possibly for another 5-10 years.

Journal papers NIM A334 (1993) 353, PR C51 (1995) 1065, and PRL 75 (1995) 2650.

Related experiments ISIS-KARMEN

E-mail contact louis@lanl.gov

WWW Home-page

http://darkmatter.linfield.edu/research/neutrino/ neutrino.html

\section{LAMPF-1188}

(Proposed 1987, Approved 1987, Began data-taking Oct 1987, In progress)

SEARCH FOR TIME REVERSAL SYMMETRY VIOLATION AND PARITY VIOLATION AT THE PROTON STORAGE RING

LOS ALAMOS - J D Bowman (Spokesperson), C M Frankle,

J N Knudson, S Penttila, S J Seestrom, V Yuan 


\section{SUMMARIES OF LOS ALAMOS EXPERIMENTS}

DUKE U -. B Crawford, N R Roberson

TRIUMF - P P J Delheij

NORTH CAROLINA STATE U - C R Gould, D G Haase,

G E Mitchell, S Patterson

DUBNA - Y Popov, E Sharapov

Accelerator LAMPF Detector Counter

Reactions Polarized beam and target

$n$ nucleus

Brief description Searches for time reversal and parity violation

in low-lying nuclear states. Preliminary results have detected parity violation in states of ${ }^{139} \mathrm{La},{ }^{165} \mathrm{Ho},{ }^{232} \mathrm{Th},{ }^{235} \mathrm{U}$, and

${ }^{238} \mathrm{U}$. The neutron beam is $57 \%$ polarized from $1 \mathrm{eV}$ to $20 \mathrm{keV}$. Continues taking data (June 96).

Journal papers PR C39 (1989) 1721, PRL 65 (1990) 1192, PRL 67 (1991) 564, PR C44 (1991) 2187, PR C45 (1992) 2143, PR C46 (1992) 768, PR C46 (1992) 778, PR C46 (1992) 1542, PR

C48 (1993) 1116, and PR C48 (1993) 1601.

E-mail contact bowman@lanl.gov

\section{LAMPF-1240}

(Proposed Jul 1991, Approved Aug 1991, Began data-taking Aug 1992, Completed data-taking Aug 1993)

MEASUREMENT OF THE MICHEL PARAMETER $\rho$ WITH THE MEGA POSITRON SPECTROMETER

MEGA COLLABORATION

CHICAGOU - S C Wrigh

FERMILAB - P S Cooper

HOUSTON U -- Y Chen, M Dzemidzic, E V IIungerford, K Lan,

B W Maycs, L Pinsky, W von Witsch

INDIANA U - J Knott, K M Stantz, J J Szymanski

LOS ALAMOS . J F Amann, R D Bolton, M D Cooper

( $\checkmark$ Spokesperson), W Foreman, $R$ Harrison, G Hart, G E Hogan,

T Kozlowski, M A Kroupa, $\mathrm{R} E$ Mischke ( $\sqrt{ }$ Spokesperson),

C Pillai, S Schilling, D Whitehouse

TEXAS A AND $M-C$ Gagliardi, $F$ Liu, $R$ E Tribble, $X L$ ' $\mathrm{C}$, I A Van Ausdeln

VALPARAISO U, INDIANA - D D Koetke, R W Manweiler. $\mathrm{S}$ Stanislaus

VIRGINIA U - B Wright, K O H Ziock

VIRGINIA TECH - D Haim, F Lee, L E Piilonen

( $\sqrt{ }$ Spokesperson), Y Zhang, W Zhou

Accelerator LAMPF Detector MEGA

Reactions Polarized beam

$$
{ }_{\mu}^{+} \rightarrow e^{+} \nu_{e} \bar{\nu}_{\mu L} \quad 28 \mathrm{MeV} / \mathrm{c}
$$

Brief description An improved measurement of the Michel parameter $\rho$. Ran for 336 hours. Data analysis in progress (July 96).

Related experiments LAMPF-969

E-mail contact mcooper@lanl.gov, mischke@lanl.gov, piilonen@amy.phys.vt.edu

\section{LAMPF-1268}

(Proposed Nov 1992, Approved Jan 1993, Began data-taking Jun 1993, Completed data-taking Sep 1993)

$\pi^{-} p \rightarrow \pi^{0} n$ CROSS-SECTIONS IN THE REGION OF THE $\triangle$ RESONANCE

ABILENE CHRISTIAN U -- L D Isenhower, J Redmon,

M E Sadler ( $\checkmark$ Spokesperson)

ARIZONA STATE U - J R Comfort, C Gaulard

BOŚKOVIĆ INST, ZAGREB - A Marušić, I Supek

CATHOLIC U - H Crannell, L Nguyen

GEORGE WASHINGTON U - W J Briscoe, J Connelley,

S Matthews

LOS ALAMOS - J Amamn, R Boudrie, J Knudson, C Morris,

B Park, M Rawool, $R$ M Whitton

PENN U - P P Hui, D Smith

Accelerator LAMPF Detector NMS

\author{
Reactions \\ $\pi^{-} p \rightarrow \pi^{0} n \quad 138,166,190,215,237,263 \mathrm{MeV}$ \\ ( $\left.\mathrm{T}_{\text {lab }}\right)$
}

Particles studied $\Delta\left(1232 P_{33}\right)$

Brief description Measures the differential cross-sections in the region of the $\Delta(1232)$ resonance. Uses elements of the Neutral Meson Spectrometer (NMS) to measure the two $\gamma$-rays from the $\pi^{0}$ decay, eliminating the difficulty of determining the efficiency of neutron counters. The goals are to provide accurate data for input to charge-dependent partial wave analyses, and to study the charge splitting of the $\Delta$.

Related experiments LAMPF-1178

E-mail.contact sadler@physics.acu.edu

\section{Other LAMPF Experiments}

Listed here are some other LAMPF experiments of interest to the particle physics community. Find more details about these projects online, in the SLAC's EXPERIMENTS database (see p. 3), or contact the spokespersons.

LAMPF-876 (Completed data-taking Oct 1992)

SPIN TRANSFER MEASUREMENTS FOR NEUTRONPROTON ELASTIC SCATTERING

Spokesperson: Michael W. McNaughton [Los Alamos]

E-mail contact mcnaught@lanl.gov

LAMPF-1178 (Completed data-taking Nov 1995)

POLARIZATION ASYMMETRY MEASUREMENTS

FOR ${ }^{1} \mathrm{H}\left(\pi^{-}, \pi^{0}\right) n$ BETWEEN 45 AND $100 \mathrm{MeV}$

Spokespersons: Joseph R. Comfort [Arizona State U.], George R. Burleson [New Mexico State U.]

E-mail contact comfort@phyast.la.asu.edu, burleson@nmsu.edu

LAMPF-1179 (Completed data-taking Jul 1992)

REACTION $\pi^{+} p \rightarrow \pi^{+} \pi^{0} p$ NEAR THRESHOLD

Spokesperson: Dinko Počanić [Virginia U.]

E-mail contact pocanic $@$ virginia.edu

WWW Home-page

http://helena.phys.virginia.edu/ pipin/E1179/ E1179.html

LAMPF-1190 (Completed data-taking Jul 1992)

PION-PROTON INTEGRAL CROSS-SECTION MEASUREMENTS

Spokespersons: Robert A. Ristinen [Colorado U.], C.L. Morris [Los Alamos]

E-mail contact ristinen@spectr.colorado.edu

LAMPF-1208 (Taking data)

NEUTRON-PROTON BREMSSTRAHLUNG

Spokesperson: Stephen A. Wender [Los Alamos]

E-mail contact wender@lanl.gov

LAMPF-1213 (Completed data-taking Nov 1995)

MEASUREMENT OF THE NEUTRINO CAPTURE CROSS-SECTION IN ${ }^{127}$ I WITH $\mu^{+}$DECAY NEUTRINOS

Spokesperson: Kenneth Lande \{Penn U.\}

E-mail contact klande@mail.sas.upenn.edu 


\section{SUMMARIES OF LOS ALAMOS EXPERIMENTS}

LAMPF-1231

LASER POLARIZED MUONIC ATOMS AND SPIN DEPENDENCE OF NUCLEAR MUON CAPTURE

Spokespersons: Gordon D. Cates [Princeton U.], Paul A. Souder [Syracuse U.]

E-mail contact cates@pucc.princeton.edu, souder@suhep.phy.syr.edu

LAMPF-1234 (Completed data-taking Sep 1991)

$K_{L L}$ AND $P$ FOR $n p$ ELASTIC SCATTERING

Spokesperson: Michael W. McNaughton [Los Alamos]

E-mail contact mcnaught@lanl.gov

LAMPF-1256 (In preparation)

$\pi^{ \pm} p$ ANALYZING POWERS AT 45 AND $67 \mathrm{MeV}$

Spokespersons: Joseph R. Comfort [Arizona State U.],

George R. Burleson [New Mexico State U.]

E-mail contact comfort@phyast.la.asu.edu, burleson@nmsu.edu

LAMPF-1267 (Completed data-taking Oct 1993)

ELASTIC SCATTERING OF $\pi^{+}$FROM POLARIZED ${ }^{3} \mathrm{He}$ AT $T_{\pi}=100,142,180$, AND $256 \mathrm{MeV}$

Spokespersons: Dietrich Dehnhard [Minnesota U.],

George R. Burleson [New Mexico State U.], Otto F. Haeusser

['ITIUMF and Simon Fraser U.]

E-mail contact dehnhard@physics.spa.umn.edu,

burleson@nmsu.edu, hausser@triumf.ca

LAMPF-1286 (Completed data-taking Sep 1993)

MEASURING THE NEUTRON-NEUTRON SCATTERING LENGTH AND EFFECTIVE RANGE USING THE

${ }^{2} \mathrm{H}\left(\pi^{-}, 2 n\right) \gamma$ REACTION

Spokesperson: Ahmed H. Hussein [Northern British Columbia U.]

E-mail contact hussein@unbc.edu

LAMPF-1293 (Completed data-taking Oct 1993)

$n p$ ELASTIC ANALYZING POWER

Spokesperson: Michael W. McNaughton [Los Alamos]

E-mail contact monaught@lanl.gov

LAMPF-1309 (Completed data-taking Oct 1993)

ANALYZING POWER AND SPIN TRANSFER MEA-

SUREMENTS IN $n p$ INELASTIC CHANNEL

Spokesperson: George Glass [Texas U.]

E-mail contact mp0gg@lampf.lanl.gov

\section{LAMPF-1310}

MEASUREMENT OF THE DOUBLY DIFFERENTYAL CROSS-SECTION FOR $\pi^{-} p \rightarrow \pi^{+} \pi^{-} n$ AT 190 AND 200 MeV AND SOFT PION THEORY

Spokespersons: Glen A. Rebka, Jr. [Wyoming U.], Pe-

ter A.M. Gram [Los Alamos], Donald A. Roberts [Michigan U.]

E-mail contact physeh@uwyo.edu, gram@lampf.lanl.gov,

droberts@mich1.physics.lsa.umich.cdu

\section{LAMPF Future Plans}

The LAMPF nuclear and particle physics user program has officially ended. The facility, now called LANSCE (Los Alamos Neutron Science Center) continues to opcrate with the purpose of providing pulsed neutrons to several research communities. However, particle physics retains its presence at Los Alamos. The LSND neutrino experiment will continue to run for at least several more years. A number of fundamental physics experiments using cold and ultracold neutrons are planned at LANSCE. 


\section{SUMMARIES OF NOVOSIBIRSK EXPERIMENTS}

\section{NOVOSIBIRSK Experiments}

\section{NOVOSIBIRSK-CMD-2}

(Proposed 1984, Approved 1985, Began data-taking 1991, In progress)

\section{THE CRYOGENIC MAGNETIC EXPERIMENT}

NOVOSIBIRSK, IYF - R R Akhmetshin, G A Aksenov,

E V Anashkin, V M Aulchenko, B O Baibusinov, V S Banzarov, L M Barkov, S E Baru, N S Bashtovoi, A E Bondar,

D V Chernyak, S I Eidelman, G V Fedotovitch, N I Gabyshev, A A Grebeniuk, D N Grigoriev, B I Khazin ( $\sqrt{ }$ Spokesperson), I A Koop, A S Kuzmin, I B Logashenko, $\mathrm{P}$ A Lukin,

A V Maksimov, Y I Merzlyakov, V S Okhapkin, S G Pivovarov, E V Popkov, T A Purlats, S I Redin, N I Root, A A Ruban, N M Ryskulov, Y M Shatunov, A I Shekhtman, A E Sher, M A Shubin, B A Shwartz, V A Sidorov, A N Skrinsky,

V P Smakhtin, I G Snopkov, E P Solodov, P Y Stepanov,

A I Sukhanov, V M Titov, Y V Yudin, V G Zavarzin,

$S$ G Zverev

BOSTON U - D H Brown, L B Roberts, W Worstell

PIT'TSBURGH U - J A Thompson

YALE U - S K Dhawan, $V W$ Hughes

Accelerator NOVO-VEPP-2M Detector CMD-2

\section{Reactions}

$$
e^{+} e^{-} \rightarrow \text { charged }^{+} \text {charged }-
$$$$
\text { (chargeds) (neutrals) }
$$

$0.36-1.4 \mathrm{GeV}\left(E_{\mathrm{cm}}\right)$

\section{Partzcles studied $\rho, \omega, \phi$}

Brief description Measures the hadronic part of the anomalous magnctic moment of the muon. Studies the dynamics of multihadron production and rare decays of vector mesons. The magnetic detector consists of a 1.5 tesla superconducting solenoid, drift chamber, Z-chamber, muon identification system, CsI barrel calorimeter, and BGO endcap calorimeter.

Journal papers NIM A252 (1986) 299, NIM A265 (1988) 137, NIM A283 (1989) 752, NIM A323 (1992) 178, and PL B364 (1995) 199.

Related experiments BNL-821

E-mail contact khazin@inp.nsk.su

WWW Home-page http://www.inp.nsk.su/cmd2/

\section{NOVOSIBIRSK-KEDR}

(Proposed 1986, Approved 1987, In preparation)

\section{THE MAGNETIC SPECTROMETER EXPERIMENT}

NOVOSIBIRSK, IYF - S Z Akhmadaliev, V V Anashin,

V M Aulchenko, B D Baibusinov, L M Barkov, A A Barladyan,

$S$ E Baru, I A Bedny, A E Blinov, G A Blinov, V E Blinov,

A E Bondar, A D Bukin, $\Lambda$ G Chilingarov, $S$ I Eideiman,

V R Groshev, G Y Kezerashviti, V A Kiselev, S G Klimenko,

G M Kolachev, V N Kozlov, L M Kurdadze, A S Kuzmin,

M Y Lelthuk, V M Malyshev, A A Maslennikov, A A Milov,

G D Minakov, SI Mishnev, N A Muchnoi, V P Nagaslaev,

E I Nekhanevich, A B Nomerotsky, A P Onuchin, V S Panin,

S V Peleganchyuk, V V Petrov, G E Pospelov, Y V Pril,

I Y Protopopov, T A Purlatz, V A Rodyakin, L V Romanov,

N I Root, A V Rylin, G A Savinov, B A Schwartz, A G Shamov,

M I Shubin, A I Shusharo, V A Sidorov, Y I Skovpen,

A N Skrinsky, V P Smakhtin, A I Sokolov, V A Tayurski,

V I Telnov, $Y$ A Tikhonov ( $\sqrt{ }$ Spokesperson), G M Tumaikin,

A E Undrus, Y V Usov, A I Vorobiev, N I Yakovlev,

I A Zagorodnikov, $\mathrm{V} N$ Zhilich, A A Zholents

BOLOGNA U \& INFN, BOLOGNA - P L Frabett

MILAN U \& INFN, MILAN - F Palombo, A Sala

PAVIA U \& INFN, PAVIA - P F Mafredi, V Re, V Speziali

Accelerator NOVO-VEPP- $4 \mathrm{M}$ Detector KEDR

$$
\begin{array}{lc}
\frac{\text { Reactions }}{e^{+} e^{-}} \rightarrow \text { hadrons } & <10.0 \mathrm{GeV}\left(\mathrm{E}_{\mathrm{cm}}\right) \\
e^{+} e^{-} \rightarrow \Upsilon(n S) & " \\
e^{+} e^{-} \rightarrow e^{+} e^{-} \gamma & " \\
e^{+} e^{-} \rightarrow e^{+} e^{-} \text {hadrons } & " \\
e^{+} e^{-} \rightarrow e^{+} e^{-} \pi^{0} & " \\
e^{+} e^{-} \rightarrow e^{-} e^{+} \eta & " \\
e^{+} e^{-} \rightarrow e^{-} e^{+} \eta^{\prime} & " \\
e^{+} e^{-} \rightarrow e^{-} e^{+} \eta_{c}(1 S) & " \\
e^{+} e^{-} \rightarrow e^{+} e^{-} a_{2}(1320) & " \\
e^{+} e^{-} \rightarrow e^{+} e^{-} \pi^{+} \pi^{-} & " \\
e^{+} e^{-} \rightarrow e^{+} e^{-} f_{2}(1270) & \text { " } \\
\text { Particles studied } \Upsilon(1 S), \Upsilon(2 S), \Upsilon(3 S), \Upsilon(4 S), \pi^{0}, \eta, \eta^{\prime}, \\
\hline \eta_{c}(1 S), a_{2}(1320), f_{2}(1270)
\end{array}
$$

Brief description Studies spectroscopy of $\Upsilon$ mesons. Particular attention is paid to a measurement of the two-photon widths of particles. The detector KEDR consists of a vertex detector, drift chambers, aerogel counter, TOF, barrel LKr calorimeter, endcap CSI calorimeter, 2-T superconducting coil, magnet yoke, muon system, and a tagging system for detection of $e^{+} e^{-}$in the twophoton processes. Schcduled to run in 1997.

Journal papers NIM A289 (1990) 468, NIM A315 (1992) 491, and NIM $A 316$ (1992) 8.

E-mail contact tikhonov@inp.nsk.su

WWW Home-page http://www.inp.nsk.su/kedr/

\section{NOVOSIBIRSK-SND}

(Proposed 1986, Approved 1987, Began data-taking 1995,

In progress)

\section{THE NEUTRAL-SPECTROMETER. EXPERIMENT}

NOVOSIBIRSK, IYF - M N Achasov, T V Baier, M G Beck, $P$ M Beschastnov, A V Bozhenok, A D Bukin, D A Bukin, S V Burdin, V P Druzhinin, M S Dubrovin, I A Gaponenko, V B Golubev, A V Gritsan, V N Ivanchenko, A A Korol, S V Koshuba, E V Pakhtusova, A A Salnikov, S I Serednyakov ( $\checkmark$ Spokesperson), V V Shary, Y M Shatunov, V A Sidorov, Z K Silagadze, A N Skrinsky, Y V Usov, Y S Velikzhanin Accelerator NOVO-VEPP-2M Detector SND

Reactions

$$
\begin{array}{ll}
e^{+} e^{-} \rightarrow \pi^{0} \gamma & <1.4 \mathrm{GeV}\left(\mathrm{E}_{\mathrm{cm}}\right) \\
e^{+} e^{-} \rightarrow \eta \gamma & " 1 \\
e^{+} e^{-} \rightarrow \omega \pi^{0} & " \\
e^{+} e^{-} \rightarrow \phi \pi^{0} & " \\
e^{++} e^{-} \rightarrow \eta \pi^{+} \pi^{-} & " \\
e^{+} e^{-} \rightarrow 4 \gamma & " \\
e^{+} e^{-} \rightarrow 5 \gamma & " \\
e^{+} e^{-} \rightarrow e^{-} e^{+} 2 \gamma & " \\
e^{+} e^{-} \rightarrow e^{-} e^{+} 3 \gamma & " \\
e^{+} e^{-} \rightarrow 2 e^{-} 2 e^{+} & " \\
e^{+} e^{-} \rightarrow 2 e^{-} 2 e^{+} \gamma & " \\
e^{+} e^{-} \rightarrow \text { pions } & " \\
e^{+} e^{-} \rightarrow 2 \text { pion } \gamma & " \\
e^{+} e^{-} \rightarrow K^{+} K^{-}, K_{S} K_{L} &
\end{array}
$$

Particles studied $K^{+}, K^{-}, K^{0}, \rho, \omega, f_{0}(975), a_{0}(980), \phi$

Brief description Studies radiative and rare decays of vector mesons, nonresonant hadronic production, and neutral kaon decays. Tests quantum clectrodynamics. 'The Spherical Neutral Detector (SND) consists of $1680 \mathrm{NaI}(\mathrm{Tl})$ counters and provides a good tool to study multiphoton events. The integrated luminosity reached $2 \mathrm{pb}^{-1}$ in the $\phi$ region as of June 96.

Journal papers YF 56-11 (1993) 75, NIM A342 (1994) 477. and NIM A361 (1995) 138.

Related experiments NOVOSIBIRSK-ND

E-mail contact serednyakov@inp.nsk.su

WWW Home-page http://www.inp.nsk.su/snd/ 
SUMMARIES OF ST. PETERSBURG EXPERIMENTS

\section{Selected PNPI Experiments}

\section{PNPI-SC-147}

(Proposed Jun 1990, Approved Jun 1990, Began data-taking Nov 1992, In progress)

STUDY OF BINARY $\pi^{-} p$ REACTIONS WITH NEUTRAL PARTICLES IN THE FINAL STATE IN THE REGION OF $N\left(1440 P_{11}\right)$ AND $N\left(1535 S_{11}\right)$ RESONANCES

PNPI-UCLA-ACU COLLABORATION

ST PETERSBURG, INP - V V Abaev, V S Bekrenev,

E A Filimonov, A B Gridnev, M R Kan, N G Kozlenko,

S P Kruglov, L V Lapochkina, I V Lopatin ( $\checkmark$ Spokesperson),

D V Novinsky, A B Starostin, VV Sumachev

UCLA - R M Clajus, B M K Nefkens

ABILENE CHRISTIAN U - L D Isenhover, M E Sadler

Accelerator PNPI Detector Counter

Reactions

$$
\begin{array}{ll}
\pi^{-} p \rightarrow n \eta & 665-715 \mathrm{MeV} / c \\
\pi^{-} p \rightarrow n \pi^{0} & 500-750 \mathrm{MeV} / c
\end{array}
$$

Brief description Measurements of DCS for the reaction $\pi^{-} p \rightarrow$ $\pi^{0} n$ in the region of $N\left(1440 P_{11}\right)$ and $N\left(1535 S_{11}\right)$ resonances, and at the $\eta$ production threshold are underway. Uses four neutron counters in coincidence with two total absorption $\gamma$ detectors. The detectors are made of C̈erenkov lead glass blocks and $\mathrm{CsI}(\mathrm{Na})$ crystals.

Journal papers PTE 1 (1995) 15, and FBS SUPPL 9 (1995) 241.

E-mail contact lopatin@lnpi.spb.su

\section{Other PNPI Experiments}

Listed here are some other PNPI experiments of interest to the particle physics community. Find more details about these projects online, in the SLAC's EXPERIMENTS database (sec p. 3), or contact the spokespersons.

PNPI-SC-124 (Completed data-taking Aug 1991)

MEASUREMENT OF THE SPIN ROTATION PARAMETERS $R$ AND $A$ IN $\pi^{-} p$ ELASTIC SCATTERING IN THE REGION OF LOW-LYING PION-NUCLEON RESO. NANCES

Spokesperson: S.P. Kruglov [St. Petersburg, INP]

E-mail contact kruglov@inpi.spb.su

PNPI-SC-129 (Completed data-taking 1992)

MEASUREMENT OF VECTOR ANALYZING POWER $i T_{11}$ IN REACTION $\pi^{-} d \rightarrow p p$ AT THE PION KINETIC ENERGY REGION 350-450 MeV

Spokesperson: A.N. Prokoficv [St. Petersburg, INP]

E-mail contact prokan@lnpi.spb.su 


\section{SUMMARIES OF VILLIGEN EXPERIMENTS}

\section{Selected PSI Experiments}

\section{PSI-R-87-01}

(Proposed Nov 1986, Approved Jan 1987, Began data-taking Dec 1988, Completed data-taking 1993)

PRECISION MEASUREMENT OF THE MUON MOMENTUM IN PION DECAY AT REST

PSI, VILLIGEN - M Daum, R Frosch ( $\sqrt{ }$ Spokesperson), P Gheno,

R Horisberger, M Janousch, P-R Kettle, C Wigger

VIRGINIA U - K Assamagan

ZURICH, ETH - H Forrer

ZURICH U - C Broennimann, T Spirig

Accelerator PSI Detector Spectrometer

Reactions

$$
\pi^{+} \rightarrow \mu^{+} \nu_{\mu} \quad 0 \mathrm{MeV} / c
$$

Particles studied $\nu_{\mu}, \pi^{+}$

Brief description Uses a surface muon beam and a magnetic spectrometer. Ran for approximately 10 weeks and measured the muon momentum to better than $4 \mathrm{ppm}$. The measurement gives an upper limit on the $\nu_{\mu}$ mass and determines the $\pi^{+}$ mass.

Journal papers PL B265 (1991) 425, PL B335 (1994) 231, and PR D53 (1996) 6065.

E-mail contact manfred.daum@psi.ch

\section{PSI-R-87-03}

(Proposed Nov 1986, Approved Jan 1987, Began data-taking 1989 In progress)

SEARCH FOR $\mu^{-} \rightarrow e^{-}$CONVERSION WITH SINDRUM II

SINDRUM-II COLLABORATION

AACHEN, TECH HOCHSCH, III PHYS INST - G Cahsor,

J Kaulard, J Kuth, G Otter, A Schnengel

PSI, VILLIGEN - W Bertl, I Egger, D Renker, J Zichy

SWIERK, INST ATOMIC ENERGY - T Kozlowski

ZURICH $U-R$ Engfer, E A Hermes, H S Pruys, F Riepenhausen,

$M$ Rutsche, A van der Schaaf ( $\checkmark$ Spokesperson), P Wintz

Accelerator PSI Detector SINDRUM-II

Reactions

$$
\mu^{-} \text {nucleus } \rightarrow e^{-} \text {nucleus } \quad 0 \mathrm{MeV} / c\left(\mathrm{P}_{\mathrm{lab}}\right)
$$

Particles studied $\mu^{-}$

Brief description The goal is to study the neutrinoless $\mu^{-} \rightarrow e^{-}$

conversion in a muonic atom, which is a test of lepton flavor conscrvation. Data were taken on $\mathrm{Ti}$ in 1989 (results are published), on $\mathrm{Pb}$ in 1992, and on $\mathrm{Ti}$ again in 1993. With the new beamline which will become available by 1997 , the sensitivity is expected to reach $10^{-14}$. Active (July 96).

Journal papers NIM A327 (1993) 378, PL B317 (1993) 631, and PRL 76 (1996) 200.

Related experiments LAMPF-969

E-mail contact vanderschaaf@psi.ch

WWW Home-page

http://www1.psi.ch/www sindrum $2_{-}$hn/sindrum2.html

\section{PSI-R-89-01}

(Proposed Jan 1989, May 1991, Approved Jan 1992, In preparation)

A PRECISE MEASUREMENT OF THE $\pi^{+} \rightarrow \pi^{0} e^{+} \nu$ DECAY RATE

PIBETA COLLABORATION
VIRGINIA U - E Frlez̆, J E Koglin, I S McCarthy, R C Minehart,

B E Norum, D Počanić ( $\checkmark$ Spokesperson), S Ritt, P L Slocum,

L C Smith, W A Stephens, B K Wright, K O H Ziock

PSI, VILLIGEN - M Daum, T Flugel, R Frosch, R Horisberger,

B R Krause, D Renker, R Schnyder

PSI, VILLIGEN \& ZURICH U - C Broennimann

SOLTAN INST, SWIERK - T Kozlowski

ARIZONA STATE U - D W Lawrence, B G Ritchie

DUBNA .. V A Baranov, V V Karpukhin, N V Khomutov,

I V Kisel, A S Korenchenko, S M Korenchenko, N P Kravchuk,

N A Kuchinsky, A Moiscenko

TBILISI STATE U ... Y Bagaturia, D Mahavia, T Sachelashvili, $\mathrm{Z}$ Tsamalaidze

BOŠKOVIĆ INST, ZAGREB - N Soić, I Supek

Accelerator PSI Detector Calorimeter, Wire chamber, Counter Reactions

$$
\begin{array}{lc}
\pi^{+} \rightarrow \pi^{0} e^{+} \nu & 0 \mathrm{MeV} / \mathrm{c} \\
\pi^{+} \rightarrow e^{+} \nu & " \\
\pi^{+} \rightarrow e^{+} \nu \gamma & "
\end{array}
$$

Particles studied $\pi^{+}$

Brief description The aim is to determine the branching ratio for the $\pi^{+} \rightarrow \pi^{0} e^{+} \nu$ decay to about $0.5 \%$ accuracy. The apparatus is a stopped-pion detector system designed to observe the two $\gamma$ 's from the $\pi^{0}$ decay, as well as the $e^{+}$. Uses a $0.75 \times 4 \pi$ pure-CsI calorimeter (consisting of 240 crystals) with a good energy resolution, MWPC's, and counters. Target is active and consists of 77 plastic scintillation fibers $3 \times 3$ $\mathrm{mm}^{2}$. Development runs are scheduled for 1996. Data taking is expected in late 1997.

Related experiments LAMPF-032

E-mail contact pocanic@virginia.edu, ritt@psi.ch WWW Home-page http://pibeta.psi.ch/

\section{PSI-R-89-06}

(Proposed Mar 1990, Approved Apr 1990, Began data-taking Jul 1992 , In progress)

SEARCH FOR SPONTANEOUS CONVERSION OF MUONIUM TO ANTIMUONIUM

HEIDELBERG U, PHYS INST .. A Grossmann, D Hubl,

$K$ Jungmann ( $\checkmark$ Spokesperson), J Merkel, V Meyer, I Reinhard,

PV Schmidt, K Trager, L Willmann. G zu Putlitz

ZURICH U - R Engfer

PSI, VILLIGEN - $\mathbb{R}$ Abela, D Renker, H K Walter

DUBNA - V Baranov, V Karpuchin, I Kisel, A S Korenchenko,

$S$ M Korenchenko, N P Kravchuk, N Kuchinsky, A Moiseenko

IBILISI STATE U - J Bagaturia, D Mzavia, T Sakelashvili

YALE U - V W Hughes

Accelerator PSI Detector SINDRUM

Reactions

$$
\mu^{+} e^{-} \rightarrow \mu^{-} e^{+} \quad 25 \mathrm{MeV} / c\left(\mathrm{P}_{\mathrm{lab}}\right)
$$

Particlesstudied $\mu^{+}$, muonium

Brief description Studies lepton number violation. 'The $\mu^{-}$

meson is detected by its decay electron, the atomic $e^{+}$is directly detected after acceleration by $8 \mathrm{kV}$. The reaction $\mu^{+} \rightarrow e^{+} e^{-} e^{+} \nu \bar{\nu}$ is also studied. Target is the $\mathrm{SiO}_{2}$ powder. First data were taken in $1992 / 93$. Expected to run till the end of 1996.

Related experiments LAMPF-1073

E-mail contact jungmann@physi.uni-heidelbcrg.de WWW Home-page

http://wwwl.psi.ch/www_mmbar_. hn/mmbar.htm

\section{PSI-R-94-01}

(Proposed Dec 1993, Approved Dec 1993, Began data-taking Jum 1994, In progress)

FEASIBILITY STUDY TO DETERMINE THE $\pi-\mu$ MASS RATIO 


\section{SUMMARIES OF VILLIGEN EXPERIMENTS}

IOANNINA U - D F Anagnostopoulos

JULICH, FORSCHUNGSZENTRUM - G Borchert, H Gorke

D Gotta ( $\checkmark$ Spokesperson), S Lenz, O W B Schult

PARIS, CURIE UNIV VI, LPAN - P El Khoury, P Indelicato

PSI, VILLIGEN - M Daum, R Frosch, P Hauser, $K$ Kirch,

L M Simons

NEUCHATEL U - M Augsburger, D Chatellard, J P Egger,

E Jeannet

Accelerator PSI Detector Spectrometer

Particles studied $\pi^{-}, \mu^{-}$

Brief description Studies $X$-rays from muonic nitrogen, muonic oxygen, and pionic nitrogen. Uses a bent crystal spectrometer. Aims to determine the $\pi^{-}$mass to $\pm 1 \mathrm{ppm}$. Taking data (July 96).

E-mail contact gotta@ikpd01.ikp.kfa-juclich.de

\section{PSI-R-94-10}

(Proposed May 1995, Approved Jun 1995, In preparation)

MEASUREMENT OF THE TRANSVERSE POLARIZATION OF POSITRONS FROM THE DECAY OF POLARIZED MUONS

ZURICH, ETH - I Barnett, C Bee, D Conti, W Fetscher

( $\sqrt{ }$ Spokesperson), M Hadri, S Kistryn, J Lang, O Naviliat,

J Sromicki, E Stephan

JAGELLONIAN U - K Bodek, L Jarczyk, J Smyrski, A Strza-

lkowski, J Zejma

Accelerator PSI Detector Drift chamber, Counter

Reactions Polarized beam

$$
\mu^{+} \rightarrow e^{+} \nu_{c} \bar{\nu}_{\mu} \quad 225 \mathrm{MeV} / c\left(\mathrm{P}_{\text {lab }}\right)
$$

Particles studied $e^{+}$

Brief description Measures two transverse polarization components, $P_{T 1}$ and $P_{T 2}$ of the positron, as a function of the $e^{+}$energy, with an expccted precision of $3 \times 10^{-3}$. This could improve by an order of magnitude the results of a previous PSI measurement of the two components, and provide a tighter limit both for the violation of timc reversal and on the value of the low-cnergy parameter $\eta$ which is used in the determination of the Fermi coupling constant $G_{F}$. Uses a high-intensity polarized muon beam, and a set of drift chambers, plastic counters, and a $\mathrm{BGO}$ array.

E-mail contact wulf.fetscher@psi.ch

\section{PSI-R-95-03}

(Proposed Nov 1994, Approved Dec 1994, Began data-taking Sep 1995, In progress)

\section{KINETIC ENERGY OF $\left(\pi^{-} p\right)$ ATOMS IN LIQUID AND} GASEOUS HYDROGEN

PSI, VILLIGEN - M Daum ( $\checkmark$ Spokesperson), W Hajdas,

P-R Kettle, V Markushin, J Schottmueller

ZURICH, ETH - R Badertscher, P F A Goudsmit, M Janousch,

Z G Zhao

Accelerator PSI Detector Counter, Calorimeter

Reactions

$$
\begin{array}{cc}
\pi^{-} p \rightarrow \pi^{0} n & 0 \mathrm{MeV} / c\left(P_{\mathrm{lab}}\right) \\
\pi^{-} p \rightarrow \gamma n & "
\end{array}
$$

Brief description Measures the time-of-night of neutrons over various fight-path lengths (from 1 to $12 \mathrm{~m}$, and possibly more) to determine the kinetic energy distribution of pionic atoms under varying pressure conditions. The same data will also be used to determine the mass difference between $\pi^{-}$and $\pi^{0}$ wit improved precision. The detection apparatus consists of counter and a NaI calorimeter.

E-mall contact manfred.daum@psi.ch

\section{PSI-R-96-05}

(Proposed Apr 1996, Approved Jun 1996, Began data-taking 1996 In progress)

\section{SEARCH FOR A NEUTRAL PARTICLE OF} MASS $=33.9 \mathrm{MeV}$ IN PION DECAY

PSI, VILLIGEN - M Daum ( $V$ Spokesperson), P-R Kettle,

$B$ Krause, J Schottmueller, O Wilhelm

PSI, VILLIGEN \& VIRGINIA U - S Ritt

'TUBINGEN U - K Fochl

ZURICH, ETH .. M Janousch, Z G Zhao

Accelerator PSI Detector Scintillator

Reactions

$$
\pi^{+} \rightarrow \mu^{+} \mathrm{X} \quad 100-150 \mathrm{MeV} / c\left(\mathrm{P}_{\mathrm{lab}}\right)
$$

Brief description Measures the muon momentum distribution in charged pion decay in flight, in order to search for a small branching fraction $\eta$ of pion decays $\pi^{+} \rightarrow \mu^{+} X$, in which a heavy neutral particle $X$, with a mass of $33.9 \mathrm{MeV}$ would be emitted. Such a particle may have been observed recently by the KARMEN Collaboration.

Journal papers PL B361 (2995) 179.

Related experiments ISIS-KARMEN

E-mail contact manfred.daum@psi.ch

\section{Other PSI Experiments}

Listed here are some other PSI experiments of interest to the particle physics community. Find more details about these projects online, in the SLAC's EXPERIMEN'TS database

(see p. 3), or contact the spokespersons. Check also the ycarly publication 'PSI Annual Report - Annex I'.

PSI-R-85-13-3 (Completed data-taking Aug 1994)

MEASUREMENT OF ELASTIC $\pi^{ \pm} p$ SCATTERING

BELOW $100 \mathrm{MeV}$

By LEPS Collaboration

Spokespersons: W. Kluge [Karlsruhe U., IEKP], II. Clement ['Tubingen U.]

E-mail contact wolfgang.kluge@physik.uni-karlsruhe.de

PSI-R-86-05 (Completed data-taking Aug 1994)

CRYSTAL DIFFRACTION OF PIONIC HYDROGEN

AND DEUTERIUM $X$-RAYS

Spokespersons: J.P. Egger [Neuchatel U.], A. Badertscher [Zurich, ETH]

E-manl contact jean-pierre.egger@iph.unine.ch. andreus, badertscher@psi.ch

PSI-R-87-12 (Completed data-taking 1995)

$n p$ ELASTIC SCATTERING: AN EXPERIMENT WITH POLARIZED NEUTRONS

Spokesperson: H. Schmitt (Freiburg U.)

E-mail contact hschmitt@uni-freiburg.de

PSI-R-89-03 (Taking data)

PIONIC DOUBLE CHARGE EXCHANGE AT LOW ENERGIES

Spokesperson: H. Clement [Tubingen U.]

E-mail contact clement@it.physik.uni-tuebingen.de 


\section{SUMMARIES OF VILLIGEN EXPERIMENTS}

PSI-R-91-08 (Completed data-taking Jul 1993) MEASUREMENT OF THE STOPPING POWER FOR MUONS $\left(\mu^{-}, \mu^{+}\right)$AT ENERGIES BETWEEN 2 AND 40 $\mathrm{keV}$

Spokespersons: F.J. Hiartmann [Munich, Tech. U.], D. Taqqu [PSI, Villigen]

E-mail contact taqqu@cvax.psi.ch

PSI-R-92-08 (Completed data-taking Sep 1992)

MEASUREMENT OF THE PRODUCTION OF THER MAL MUONIUM IN VACUUM FROM SILICA AERO-

GELS

Spokespersons: K. Jungmann, B. Matthias [Heidelberg U., Phys. Inst.]

PSI-R-93-06 (In preparation)

MEASUREMENT OF THE $3 d-3 p$ TRANSITION IN MUONIC HYDROGEN WITH A COMPACT WAVEGUIDE FREE-ELECTRON LASER

By MUH Collaboration

Spokesperson: E. Zavattini [Trieste U.]

E-mail contact milotti@dfists.ts.infn.it

PSI-R-95-08 (Taking data)

SPIN DEPENDENCE OF PION PRODUCTION IN NEUTRON-PROTON COLLISIONS

Spokespersons: H. Schmitt [Frciburg U.], M. Finger [Charles U. and Dubna, JINR]

E-rnail contact hschmitt@uni-freiburg.de

PSI-R-95-09

MEASUREMENT OF THE DESTRUCTIVE INTERFERENCE OF $s$ AND $p$ WAVE IN $\pi^{-} p$ ELASTIC SCATTER-

ING AT $180^{\circ}$

Spokesperson: M. Janousch [Zurich, ETH]

E-mazl contact markus.janousch@psi.ch

PSI-Z-89-02 (Completed data-taking Jun 1991)

NEUTRON MAGNETIC FORM-FACTOR

Spokesperson: Juerg Jourdan (Basel U.)

E-mazl contact jourdan@urz.unibas.ch

PSI-Z-89-06 (Completed data-taking 1991)

SPIN DEPENDENT TOTAL CROSS-SECTION $\Delta \sigma_{L}$ IN $n p$ SCATTERING

Spokesperson: P. Haffter [Basel U.]

E-mail contact jourdan@urz.unibas.ch

PSI-Z-89-07 (Completed data-taking 1991)

$\vec{n} p$ RADIATIVE CAPTURE

Spokesperson: G.S. Masson [Basel U.]

$\underline{E-m a i l ~ c o n t a c t}$ masson@urz.unibas.ch

PSI-Z-90-07 (Taking data)

SEARCH FOR EXTENSIONS OF THE STANDARD

MODEL BY A RELATIVE $\beta$-POLARIZATION MEA-

SUREMENT FROM POLARIZED NUCLEI

Spokesperson: Oscar Naviliat-Cuncic [Zurich, E'TH]

E-mail contact naviliat@imp.phys.ethz.ch
PSI-Z-90-12 (Taking data)

DEVELOPMENT OF A SUPERCONDUCTING NEUTRINO AND DARK MATTER DETECTOR

Spokesperson: Klaus Pretzl [Bern U.]

E-mail contact pretzl@cernvm.cern.ch

PSI-Z-91-02 (Completed data-taking 1992)

MEASUREMENT OF THE NEUTRON-PROTON SPIN CORRELATION PARAMETER AT FORWARD ANGLES

Spokesperson: Benny Zihlmann [Basel U.]

E-mail contact ziblmann@urz.unibas.ch

\section{PSI Future Plans}

PSI plans to continue its development and operation of large, complex accelerator facilities. The Spallation Neutron Source (SINQ) will begin operations shortly, and other facilities, e.g., the Swiss Light Source (SLS) are in the planning stage. Although the emphasis placed earlier on nuclear and particle physics has shifted more towards solid state physics and materials sciences over the last few years, the nuclear/particle physics programs can benefit largely from the diversification of accelerator usage. Accordingly, new large particle physics experiments (e.g., the rare decay experiments such as $\mu \rightarrow e \gamma)$, are envisaged in the long term plans at PSI. The near-future plans include a continuing study of rare decays and exotic atoms, the $\mu \mathrm{SR}$ experiments, as well as the exploitation of very slow muons. 


\section{SUMMARIES OF SACLAY EXPERIMENTS}

\section{Selected SATURNE Experiments}

\section{SATURNE-186}

(Proposed Oct 1987, Approved Dec 1987, Began data-taking 1988, Completed data-taking 1993)

STUDY OF HEAVY MESON PRODUCTION IN REACTIONS $p d \rightarrow{ }^{3} \mathrm{HeX}$ AND $d d \rightarrow{ }^{4} \mathrm{HeX}$

STRASBOURG, CRN - O Bing, F Hibou

SACLAY - J Arvieux, M Boivin, J M Durand, F Plouin

ORSAY, IPN - L Bimbot, M P Comets, P Courtat, Y Le Bornec

(Spokesperson), E Loireleux, $\mathrm{F}$ Reide, B Tatischeff, $\mathrm{N}$ Willis

Accelerator SATURNE-II Detector SPES-III

Reactions

$$
\begin{array}{ll}
p \text { deut } \rightarrow{ }^{3} \mathrm{He} \mathrm{X} & 900-2700 \mathrm{MeV}\left(\mathrm{T}_{\mathrm{lab}}\right) \\
\text { deut deut } \rightarrow{ }^{4} \mathrm{HeX} & 1150-2150 \mathrm{MeV}\left(\mathrm{T}_{\mathrm{lab}}\right)
\end{array}
$$

Particles studied deut, ${ }^{3} \mathrm{He},{ }^{4} \mathrm{He}$, meson

Brief description Studies the heavy meson production at proton energies between 900 and $2700 \mathrm{MeV}$, and deuteron energies 1150 and $2150 \mathrm{MeV}$. Measures the angular distribution.

Journal papers Paper to be published soon.

Related experiments SATURNE-253

E-mail contact le_bornec@ipncls.in2p3.fr

\section{SATURNE-198}

(Proposed Mar 1988, Approved Dec 1988, Began data-taking Nov 1990, Completed data-taking 1991)

\section{MEASUREMENTS OF SOME RARE DECAY MODES OF THE $\eta$}

SACLAY - A Baldisseri, A Boudard, B Fabbro, M Garcon, W Jacobs, C Kerboul, B Mayer ( $\checkmark$ Spokesperson), I Poitou, 3 Saudinos, E Tomasi, S Vigdor, F Wellers

UCLA - R Kessler, BMK Nefkens ( $\checkmark$ Spokesperson), B Tippens, $M$ Wang

ZURICH U - E A Hermes, C Niebuhr, A van dex Schaaf GEORGE WASHINGTON U - W Briscoe, A Petrov

TRIUMF - R Abegg, W T H van Oers

DUBNA - L Lytkin

Accelerator SATURNE-II Detector SPES-II

Reactions

$$
p \text { deut } \rightarrow{ }^{3} \text { He } \eta \quad>896 \mathrm{MeV}\left(\mathrm{T}_{\text {lab }}\right)
$$

Particles studied $\eta$

Brief description Measures the $\eta$ branching ratio to $\mu^{+} \mu^{-}$ with $12 \%$ accuracy. The muons are detected by a two-range telescope. Events are identified by using constraints like coplanarity, angular correlation, total energy conservation, and the invariant mass of the two muons.

Journal papers PRL 70 (1993) 892, PR D50 (1994) 92, PR C53 (1996) 2068, and PR D53 (1996) 6658

\section{Related experiments SATURNE-258}

E-mail contact mayer@phnx7.saclay.cea.fr, bnefkens@uclapp.physics.ucla.edu

\section{SATURNE-209}

(Proposed Mar 1989, Approved 1989, Began data-taking 1990, Completed data-taking Nov 1991)

CROSS-SECTION AND ASYMMETRIES FOR THE $p p \rightarrow p p \pi^{0}$ REACTION FROM THRESHOLD TO $1 \mathrm{GeV}$

BONN U - G Anton, J Arends, M Brcuer, K Buchler,

$P$ Hoffmann-Rothe, G Nocldeke
SOUTH CAROLINA U - G Blanpied ( $\sqrt{ }$ Spokesperson), $B$ Preedom

ORSAY, IPN - G Berrier-Ronsin, J P Didelez ( $\checkmark$ Spokesperson), A Elayi, R Frascaria, E Hourani ( $\sqrt{\text { Spokesperson) }}$

G Rappenecker, M Rigney, L Rosier

SACLAY - J M Laget, B Saghai

Accelerator SATURNE-II Detector SPES-0

Reactions Polarized beam

$$
p p \rightarrow p p \pi^{0} \quad 325-1000 \mathrm{MeV}\left(\mathrm{T}_{\mathrm{lab}}\right)
$$

Brief description Measures the differential, total cross-sections and beam asymmetries for the $\pi^{0}$ emitted in the reaction, from threshold to $1000 \mathrm{MeV}$. Uses the SPESO-2 $\pi$ Neutral Meson Spectrometer and a liquid $\mathrm{H}_{2}$ target with polarized protons as projectiles.

Journal papers NP A590 (1995) 763.

Related experiments SATURNE-129, SATURNE-132, SATURNE-134, SATURNE-155

$\underline{E-m a i l ~ c o n t a c t}$ didelez@ipncls.in2p3.fr

\section{SATURNE-213}

(Proposed 1989, Approved 1989, Began data-taking 1994, In progress)

MEASUREMENT OF SPIN DEPENDENT OBSERV. ABLES IN THE REACTION $p p \rightarrow p K^{+} Y^{*}$

DISTO COLLABORATION

INDIANA U - L C Bland, S Choi, M Dzemidzic, W W Jacobs, $S$ E Vigdor

TURIN U \& INFN, TURIN - F Balestra, $S$ Bossolasco,

M P Bussa, L Fava, L Ferrero, R Garfagnini, A Grasso,

A Maggiona, D Panzieri, G Piragino, F Tosello, G Zosi

DUBNA - I Falomkin, V Frolov, V Ivanov, G B Pontecorvo,

A Popov, V Tchalyshev, B Zalikhanov

SACLAX - Y Bedfer, R Bertini ( $\checkmark$ Spokesperson), F Brochard, J C Faivre, A Manara

DARMSTADT, GSI - M Debowski, P Senger

CRACOW \& JAGELLONIAN U - J Foryciarz, P Salabura

GIESSEN $U$ - A Brenschede, W Kuhn, H Pfaff

FRANKFURT U - J Stroth

ROSSENDORF, FORSCHUNGSZENTRUM - E Grosse

TRIUMF D Gill

Accelerator SATURNE-II Detector DISTO

Reactions Polarized beam

$$
\begin{aligned}
& p p \rightarrow p K^{+} \Lambda \\
& p p \rightarrow p K^{+} \Sigma^{0} \\
& p p \rightarrow p K^{+} Y^{*} \text { (unspec) } \\
& p p \rightarrow p p K^{+} K^{-} \\
& p p \rightarrow p p K^{+} K^{-} \phi
\end{aligned}
$$

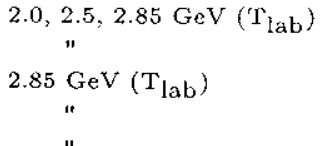

Brief description The experiment measures differential crosssections and spin-dependent observables (analyzing power, polarization and depolarization) between the threshold and the highest energy available at SATURNE-II. Studies a correlation between the measured observables and $N^{*}$ and $Y^{*}$ resonances. Uses a liquid hydrogen target. The detector, DISTO, consists of a magnet, scintillating fiber detectors, MWPC's, scintillation hodoscope and a water Cerenkov hodoscope. In the next year's run, a replacement of the liquid hydrogen target by a nuclear target is envisaged in order to study the interaction of $Y$ and $Y^{*}$ with nuclear matter. Taking data (July 96).

Journal papers NP A585 (1995) 265c.

E-mail contact bertini@frcpnl1.in2p3.fr

WWW Home-page

http://www.to.infn.it/esperimenti/disto/disto.html 


\section{SUMMARIES OF SACLAY EXPERIMENTS}

\section{SATURNE-220}

(Proposed Jun 1990, Approved Dec 1990, Began data-taking Mar 1991, Completed data-taking 1991)

SEARCH FOR THE EXCITATION OF THE ROPER RESONANCE (1440) BY INELASTIC SCATTERING OF $\alpha$ PARTICLES

SACLAY - M Boivin, H P Morsch (Spokesperson), F Plouin, B Saghai, J Yonnet, P Zupranski

ORSAY, IPN -- J P Didelez, R Frascaria (Spokesperson),

M Morlet, R Siebert, E Warde

JULICH, FORSCHUNGSZENTRUM \& STOCKHOLM U -

P E Tegner

Accelerator SATURNE-II Detector SPES-IV

Reactions

${ }^{4} \mathrm{He} p \rightarrow{ }^{4} \mathrm{HeX} \quad 4.2 \mathrm{GeV}\left(\mathrm{T}_{\mathrm{lab}}\right)$

Particles studied $N^{*}$ (unspec), $N\left(1440 P_{11}\right)$

Brief description Studies the baryon excitation in the $\alpha p$ system, from the pion threshold up to the Roper resonance. Uses the $\alpha$ beam up to $7 \mathrm{GeV} / c$. Inelastically scattered alpha particles are detected by the SPES-IV spectrometer. Uses LH2 target.

Journal papers PRL 69 (1992) 1336, and ZPHY A348 (1994) 45. Related experments SATURNE-251

E-mail contact morsch@frcpn11.in2p3.fr, frascaria@ipncls.in 2p3.fr

\section{SATURNE-222}

(Proposed Nov 1989, Approved 1989, Began data-taking 1990, Completed data-taking 1993)

MESON PRODUCTION NEAR THRESHOLD FROM THE $\phi$ TO THE $f_{1}(1285)$

ORSAY, IPN -- J P Didelez, M A Duval, R Frascaria, M Morlet, $R$ Siebert (Spokesperson), E Warde

SACLAY - J Arvieux, F Plouin

BONN U - J Bisplinghoff, J Ernst, $F$ Hinterberger, $R$ Jahn

(Spokesperson), R Joosten, U Lahr, C Lippert, A Marx, R Wurzinger

Acceleralor SATURNE-II Detector SPES-IV

Reactions

$p$ deut $\rightarrow{ }^{3} \mathrm{He} \mathrm{X}$

Particles studied $\phi, f_{1}(1285)$

Brief description Extends and refines existing measurements of the threshold excitation curve of meson production. An increasing degree of cxclusivity is achicved by adding scintillator arrays to the SPES-IV detector. In the second phase, during $1992 / 93$, the regions around the $K^{+} K^{0}$ threshold, and above $1.9 \mathrm{GeV}$, are explored in $100-\mathrm{McV}$ steps.

Journal papers PR C51 (1995) 443.

E-mail contact siebert@ipncls.in2p3.fr

\section{SATURNE-225}

(Proposed Dec 1989. Approved Jun 1991, Began data-taking Nov 1991, Completed data-taking Apr 1995)

DETERMINATION OF THE NUCLEON-NUCLEON SCATTERING AMPLITUDES IN THE ENERGY REGION FROM 1.1 TO $2.7 \mathrm{GeV}$ AND A SEARCH FOR A STRUCTURE AROUND $T_{\mathrm{kin}}=2.1 \mathrm{GeV}$

NUCLEON-NUCLEON COLLABORATION

SACLAY - I Arvieux, J Ball ( $\checkmark$ Spokesperson), J Bystricky, $P$ A Chamonard, $M$ Combet, $A$ de Lesquen, $M$ de Mali,

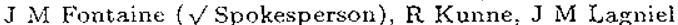

F Lehar. J L Lemaire, J L Sans
GENEVA U - P Demierre, R Hess, Z F Janout, Jr, D Rapin ( $\checkmark$ Spokesperson), A Teglia, B Vuaridel

ARGONNE - C Allgower, M Beddo, D Grosnick, T Kasprzyk, D Lopiano, H Spinka ( $\sqrt{ }$ Spokesperson)

DUBNA - L S Barabash, V A Kalinnikov, Y M Kazarinov

B A Khachaturov ( $\sqrt{ }$ Spokesperson), $V \mathrm{~N}$ Matafonov,

I L Pisarev, $A$ A Popov, Y A Usov

DUBNA \& PRAGUE, TECH U - Z Janout

ST PETERSBURG, INP - A N Prokofiev, V Vikhrov,

A A Zhdanov

UCLA - A Boutefnouchet, C M Dulya, V Ghazikhanian,

$\mathrm{S}$ Trentalange, $\mathrm{C}$ A Whitten

MIT, LNS - E L Lomon

Accelerator SA'TURNE-II Detector Combination

Reactions Polarized beam and target

$p p \rightarrow p p \quad 1.1-2.8 \mathrm{GeV}\left(\mathrm{T}_{\mathrm{lab}}\right)$

$p n \rightarrow p n \quad 1.1-2.4 \mathrm{GeV}\left(\mathrm{T}_{\mathrm{lab}}\right)$

Particles studied $p, n$

Brief description Uses a polarized proton beam and polarized proton and deuteron targets. Measures the complete sets of spin-dependent obscrvables in pn quasielastic scattering between 1.1 and $2.4 \mathrm{GeV}$. Dedicated $p p$ spin-dependent observables are measured between 1.8 and $2.8 \mathrm{GeV}$ in small steps of energy. The measuring apparatus consists of a two-arm spectrometer, one arm being a polarimeter, and two large neutron-counter hodoscopes. The direction of beam polarization is measured by three additional beam polarimeters. Data analysis in progress (July 96)

Journal papers NIM A327 (1993) 308, and PL B320 (1994) 206.

Related experiments SATURNE-144, SATURNE-216

E-mall contact ball@frcpn11.in2p3.fr, jmfont@frcpn11.in2p3.fr, hms@hep.anl.gov, khachaturov@main1.jinr.dubna.su

\section{SATURNE-237}

(Proposed Nov 1990, Approved Jan 1991, Began data-taking Jul 1991, Completed data-taking 1995)

STUDY OF THE $p p \rightarrow p p \eta$ AND $(p, \eta)$ REACTIONS ON NUCLEI AT $T_{p}>1.26 \mathrm{GeV}$

PINOT COLLABORATION

TURIN U \& INFN, TURIN - E Chiavassa, N De Marco

(Spokesperson), C De Oliveira Martins, G Dellacasa, F Ferrero,

M Gallio, P Guaita, A Musso, A Piccotti, E Scomparin,

E Vercellin (Spokesperson)

SACLAY - J M Durand, G Milleret

Accelerator SATURNE-II Detector PINOI

Reactions

$$
\begin{array}{lc}
p p \rightarrow p p \eta & >1.26 \mathrm{GeV}\left(\mathrm{T}_{\text {lab }}\right) \\
p \text { nucleus } \rightarrow \eta \mathrm{X} & " \\
p \text { deut } \rightarrow \eta \mathrm{X} & "
\end{array}
$$

\section{Particles studied $\eta$}

Brief description The aim is to study the first two reactions near the threshold by detecting $\eta$ mesons with the two-arm neutral meson spectrometer, PINOT. For the first reaction the total and doubly differential cross-section $d^{2} \sigma / d \Omega d T$ is measured. The $(p, \eta)$ reaction on nuclei is studied at the same incident energies by measuring the $\eta$ kinetic energy distributions for $\eta$ 's emitted forward in the laboratory. Also investigates the $p d \rightarrow \eta X$ reaction in order to extract information on the $p n \rightarrow \eta X$ elementary process, by comparing $p d$ and $p p$ induced reactions. Uses the following targets: liquid $\mathrm{H}_{2}$ and $\mathrm{D}_{2}, \mathrm{C}, \mathrm{Al}$, $\mathrm{Cu}, \mathrm{Mo}, \mathrm{W}, \mathrm{Au}$, and $\mathrm{Yi}$. Data analysis in progress (July 96).

Journal papers NP A538 (1992) 121c, ZPHX A342 (1992) 107, JPHY G19 (1993) L51, NC 106A (1993) 861, ZPHY A344 (1993) 345, NC 107A (1994) 1195. PL B322 (1994) 270, and PL B337 (1994) 192.

Related experiments SATURNE-125

E-mail contact demarco@to.infn.it, verccllin@to.infn.it 


\section{SUMMARIES OF SACLAY EXPERIMENTS}

\section{SATURNE-251}

(Proposed Nov 1992, Approved Dec 1992, Began data-taking Mar 1993, Completed data-taking Oct 1993)

\section{SEARCH FOR THE EXCITATION OF THE ROPER} RESONANCE (1440) IN NUCLEI

SACLAY - M Boivin, J L Boyard (Spokesperson), F Fuchs,

R Kunne, H P Morsch (Spokesperson), F Plouin, P Radvanyi, W Spang

ORSAY, IPN - T Hennino, J C Jourdain, B Ramstein, M Roy-

Stephan, S Rusteau

IULICH, FORSCHUNGSZENTRUM -- V Jaeckle

WARSAW U - P Zupranski

STOCKHOLM U - P E Tegner

RENSSELAER POLY - L Murphy, P Stoler

Accelerator SATURNE-II Detector SPES-IV

Reactions

${ }^{4} \mathrm{He}$ deut $\rightarrow{ }^{4} \mathrm{He} \mathrm{X} \quad 4.2 \mathrm{GeV} / \mathrm{c}$

${ }^{4} \mathrm{He}{ }^{12} \mathrm{C} \rightarrow{ }^{4} \mathrm{HeX}$

Particles studied $N\left(1440 P_{11}\right)$

Brief description Studies the spectrum of alpha particles.

Searches for the excitation of the Roper resonance. Uses LD2,

solid $\mathrm{C}$, and $\mathrm{CH}_{2}$ targets. Data analysis in progress (July 96).

Related experiments SATURNE-220

E-mail contact boyard@frcpn11.in2p3.fr, morsch@frcpn $11 . i n 2 p 3 . f r$

\section{SATURNE-258}

(Proposed Nov 1992, Approved Dec 1992, Began data-taking 1993, Completed data-taking 1993)

DIRECT MEASUREMENT OF THE BRANCHING RATIO IN THE $\eta \rightarrow \gamma \gamma$ DISINTEGRATION

ETA COLLABORATION

SACLAY - A Boudard, J M Durand, B Fabbro, M Garcon

( $\checkmark$ Spokesperson), B Mayer, J F Pillot, E Thomasi-Gustafsson

DUBNA - A Efendiev, L Lytkin ( $\sqrt{ }$ Spokesperson)

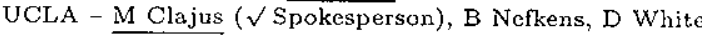

PSI, VILLIGEN - R Abela

TRIUMF - $\mathrm{R}$ Abegg, P Fuchs, W $\mathrm{T} H$ van Oers

GEORGE WASHINGION U - W Briscoe, $T$ Morrison

ST PETERSBURG, INP - V Nikulin

Accelerator SATURNE-II Detector Calorimetcr, SPES-IY

Reactions

$p$ deut $\rightarrow{ }^{3} \mathrm{He} \eta \quad 894 \mathrm{MeV}\left(\mathrm{T}_{\text {lab }}\right)$

Particles studied $\eta, \gamma$

Brief descraption The first direct measurement of the branching

ratio $\Gamma(\eta \rightarrow \gamma \gamma) / \Gamma_{\text {tot }}$. Expected to achieve an accuracy of 2

to $2 \%$. Uses two BGO photon calorimeters and SPES-II. The

target is LD2. Data analysis completed.

Journal papers PR D53 (1996) 11. No other papers expected.

Related experiments SATURNE-198, SATURNE-284

E-mail contact clajus@uclapp.physics.ucla.edu

garcon@phnx7.saclay.cea.fr, lytkin@main\}.jinr.dubna.su

\section{SATURNE-278}

(Approved Jun 1993, Began data-taking Dec 1995, In progress) STUDY OF BARYONIC RESONANCES WITH THE NEW DETECTOR AROUND THE SPES-IV TARGET POINT

SPES4-PI COLEABORATION

COPENHAGEN U - R Dahl, C Ellegard, C Gaarde, J Jensen.

J S Larsen, M Skousen
DUBNA - Y T Borzunov, L P Golovanov, V P Ladygin, L V Malinina, N M Piskunov, I M Sitnik, E A Strokovsky ( $\sqrt{ }$ Spokesperson), A P Tsvinev

ST PETERSBURG, INP - G D Alkhazov, A V Khanzadeyev, V A Mylnikov, A N Prokofiev, V M Samsonov, I V Tkach, $\checkmark \mathrm{N}$ Vikhrov, $A$ A Vorobyov, A A Zhdanov

JULICH, FORSCHUNGSZENTRUM - H P Morsch

NORFOLK STATE U - V Punjabi

ORSAY - L Bimbot, J L Boyard ( $\sqrt{ }$ Spokesperson), L Farhi, J C Jourdain, B Ramstein, M Roy-Stephan

SACLAY - M Boivin, T Hennino, M Kagarlis, R A Kunne ( $\checkmark$ Spokesperson), P Radvanyi, E Tomasi-Gustafsson

WARSAW, INST NUCL STUDIES - W Augustyniak, P Zupransk

WILLIAM AND MARY COLL - M Jones, C F Perdrisat

Accelerator SATURNE-II Detector SPES4-PI

Reactions

$$
\begin{array}{lc}
\text { deut } p \rightarrow \text { deut } p \mathrm{X} & <3.8 \mathrm{GeV} / \mathrm{c} \\
{ }^{3} \mathrm{He} \text { nucleus } \rightarrow \text { trit } \pi^{+} \mathrm{X} & " \\
{ }^{4} \mathrm{He} p \rightarrow{ }^{4} \mathrm{He} p \mathrm{X} & <7.0 \mathrm{GeV} / c \\
{ }^{12} \mathrm{C} \text { nucleus } \rightarrow{ }^{12} \mathrm{~N} \pi^{-} \mathrm{X} & <3.8 \mathrm{GeV} / \mathrm{c}
\end{array}
$$

Particles studied $N^{*}$ (unspec), $N\left(1440 P_{11}\right)$

Brief description This is a three-part experiment $(278 \mathrm{a}$,

$278 \mathrm{~b}, 278 \mathrm{c}$ ), carried out with a now detector at the target point of SPES-IV. The detector consists of the SPES-IV high resolution spectromcter to detect the scattered bcam particles in coincidence with the secondary protons and pions which are registered by two arms of chambers. Studies baryonic resonances. Uses polarized and unpolarized beams, and $\mathrm{C}, \mathrm{Ca}$, and $\mathrm{Pb}$, targets. The topics covered by the three parts are (1) coherent pion production from charge exchange reactions, (2) production of the $N^{*}(1440)$ resonance with alpha particles, and (3) study of spin observables in the production of the $N^{*}(1440)$ resonance with deuterons. In progress (July 96).

Related experiments SATURNE-250, SATURNE-291,

SATURNE-305, JINR-LIIE-0941-1B, JINR-LHE-0941-1C

E-mail contact boyard@frcpn11.in2p3.fr,

strok@sunhe.jinr.dubna.su, kumne@frcpn11.in2p3.fr

\section{Other SATURNE Experiments}

Listed here are some other Saclay experiments of interest to the particle physics community. Find more details about these projects online, in the SLAC's EXPERIMENTS database (see p. 3), or contact the spokespersoris.

\section{SATURNE-121 (Completed data-taking 1991) \\ SEARCH FOR DIBARYONS OF STRANGENESS $S=-1$ BETWEEN THE $A N$ AND $\Sigma N$ THRESHOLDS \\ Spokespersons: J.P. Didelez, R. Frascaria [Orsay, IPN], B. Pree- dom [South Carolina U.]}

$\underline{\text { E-mail contact }}$ didelez@ipncls.in2p3.fr, frascaria@ipncls.in2p3.fr

SATURNE-144 (Completed data-taking Dec 1990)

NUCLEON-NUCLEON PROGRAM (PART II): $n p$ SCATTERING UP TO $1.2 \mathrm{GeV}$

Spokcspersons: F. Lehar, F. Perrot [Saclay], R. Hess [Geneva U.]

E-mail contact lehar@frcpn11.in2p3.fr, hess@sc2a.unige.ch

SATURNE-145 (Completed data-taking 1990)

MEASUREMENTS OF $A_{z z}$ AND $P_{z}$ FOR THE REACTION $\overrightarrow{d p} \rightarrow \vec{p} p n$ IN COMPLETE KINEMATYCS

Spokespersons: S.L. Belostotsky [St. Petersburg, INP], A. Boudard [Saclay]

E-mail contact boudard@phnx7.saclay.cea.fr 


\section{SUMMARIES OF SACLAY EXPERIMENTS}

SATURNE-166 (Completed data-taking 1991)

REACTION HI $(d, 2 p) n$ WITH POLARIZED DEUTERONS AT $200 \mathrm{MeV}$

Spokesperson: S. Kox [Grenoble U.]

E-mail contact kox@frcpnd1.in $2 \mathrm{p} 3 . \mathrm{fr}$

SATURNE-174 (Completed data-taking)

PRODUCTION OF LIGHT MESONS $X$ IN $p p \rightarrow p p X$ AT THRESHOLD AND IN NUCLEAR MATTER

Spokesperson: O. Bing [Strasbourg, CRN]

E-mail contact obing@frcpn11.in2p3.fr

SATURNE-190 (Completed data-taking May 1991)

SPIN STRUCTURE OF THE $\triangle$ EXCITATION

Spokespersons: C. Gaarde [Copenhagen U.], J.L. Boyard [Orsay], P. Zupranski [Soltan Inst., Swicrk]

E-mail contact gaarde@nbivax.nbi.dk, zupran@fuw.edu.pl

SATURNE-202 (Completed data-taking 1992)

STUDY OF THE PROTON POLARIZATION IN $\overrightarrow{d A} \rightarrow \vec{p} X$ REACTION AT $0^{\circ}$ AND $2.1 \mathrm{GeV}$

Spokespersons: C.F. Perdrisat [William and Mary Coll.], J. Yonnet [Saclay]

E-mail contact yonnet@frcpn11.in2p3.fr, perdrisat@cebaf.gov

SATURNE-235 (Completed data-taking 1993)

CALIBRATION OF POLDER, A NEW DEUTERON TENSOR POLARIMETER AT INTERMEDIATE ENERGIES

Spokesperson: Serge Kox [Grenoble U.]

E-mail contact kox@frcpn1t.in2p3.fr

SATURNE-244 (Completed data-taking Sep 1991)

STUDY OF THE REACTION $\vec{p} p \rightarrow \pi^{-} \pi^{-} X$

Spokesperson: B. Tatischeff [Orsay, IPN]

E-mail contact tati@frcpn11.in2p3.fr

\section{SATURNE-246 (Completed data-taking 1992)}

$\pi^{0}$ PRODUCTION IN THE REACTION $d p \rightarrow{ }^{3} H e \pi^{0}$ NEAR THRESHOLD

Spokespersons: B. Mayer [Saclay], B.M.K. Nefkens [UCL $A$ ]

E-mail contact mayer@phnx7.saclay.cea.fr,

bnefkens@uclapp.physics.ucla.edu

SATURNE-249 (Completed data-taking 1993)

POLARIZATION TRANSFER IN ELASTIC BACKWARD DEUTERON PROTON SCATTERING

Spokespersons: C.F. Perdrisat [William and Mary Coll.], V. Punjabi [Norfolk State U.], I. Sitnik [Dubna, JINR]

E-mail contact perdrisat@cebaf.gov, punjabi@cebaf.gov,

sitnik@lhe06.jinr.dubna.su
SATURNE-253 (Completed data-taking 1992)

MEASUREMENTS OF THE POLARIZATION TENSOR AND THE PROBABILITY OF THE SPIN-FLIP IN THE REACTION ${ }^{12} \mathrm{C}\left(d, d^{\prime}\right)^{12} \mathrm{C}$ AT $400 \mathrm{MeV}$

Spokespersons: S. Kox [Grenoble U.], E. Tomasi-Gustafsson [Saclay], M. Morlet [Orsay, IPN]

E-mail contact kox@frcpn11.in2p3.fr, tomasi@chatelet.saclay.cea.fr

SATURNE-280 (Taking data)

STUDY OF THE REACTION $d d \rightarrow \alpha \eta$ CLOSE TO THE THRESHOLD OF $\eta$ PRODUCTION

Spokespersons: $\Lambda$. Zghiche [Strasbourg, CRN], R. Wurzinger

[Saclay], L. Lytkin [Dubna, JINR], Y. Le Bornec, N. Willis [Orsay, IPN]

$\underline{E-m a i l \text { contact }}$ le_bornec@ipncls.in2p3.fr, willis@ipncls.in2p3.fr

SATURNE-290 (Completed data-taking 1994)

MEASUREMENT OF TENSOR OBSERVABLES

RELATED TO THE POLARIZATION OF RECOIL

DEUTERON IN THE REACTION $p p \rightarrow d \pi^{+}$

Spokespersons: C. Furget, S. Kox [Grenoble U.]

E-mail contact kox@frcpni1.in2p3.fr

\section{SATURNE Future Plans}

The SATURNE-II accelerator, best known for its polarized proton and deuteron beams, will be closed by the end of 1997. The strong physics program involving large international participation has now been reduced to just two experiments (213 and 278) which are studying the baryonic resonances and a production of coberent pions in the charge exchange reactions. Other experiments and important $R \& D$ projects have either been completed or cancclled. There are no plans for direct experimental research at the site beyond 1997 , but the Lab will be involved in many intermediateand high-energy physics projects at other facilities. 


\section{SUMMARIES OF SERPUKHOV EXPERIMENTS}

\section{Selected Serpukhov Experiments}

\section{SERPUKHOV-128}

(Proposed 1977, Approved 1984, Began data-taking 1987)

SEARCH FOR NEW SHORT-LIVED PARTICLES IN NEUTRINO INTERACTIONS

SERPUKHOV - V V Ammosov, V I Baranov, A A Ivanilov,

P V Ivenov, V M Korablev, V A Korotkov, V V Makeev,

A G Myagkov, P V Pitukhin, A Y Polyarush, A A Sokolov

MOSCOW PHYS ENG INST - E Gushchin, A I Lebedev,

S V Somov (Spokesperson), G I Tipografshchik

MOSCOW, ITEP - Y A Aleshin, O K Egorov, E D Kolganova

A N Maksimov, I A Melnichenko, E A Pozharova, V I Silaev,

V A Smirnitsky, V A Smotryaev, I S Trostin

LEBEDEV INST - S I Kotelnikov, E P Kuznetsov,

B I Lomonosov, L I Pervov, V A Ryabov, P S Vásiliev

MOSCOW STATE U - P F Ermolov, V S Murzin, S I Sivoklokov

DUBNA - Y A Batusov, S A Bunyatov, O M Kuznetsov,

V V Lyukov, V I Tretyak

Accelerator SERPUKHOV Detector Combination

\section{Reactions}

$$
\nu_{\mu} \text { nucleon } \rightarrow \mu^{-} \text {charm X }
$$

$\nu_{\mu}$ nucleon $\rightarrow \Lambda_{c}^{+} \mu^{-} \mathrm{X}$

$\nu_{\mu}$ nucleon $\rightarrow \Sigma_{c}(2455)^{+} \mu^{-} \mathrm{X}$

$\nu_{\mu}$ nucleon $\rightarrow \Sigma_{c}(2455)^{++} \mu^{-} \mathrm{X}$

$\nu_{\mu}$ nucleon $\rightarrow \mu^{-}$charmed-meson $\mathrm{X}$

$\nu_{\mu}$ nucleon $\rightarrow D_{s}^{ \pm} \mu^{-} \mathrm{X}$

Particles studied charm

Brief description The detector is a wide angle spectrometer with a streamer chamber and emulsions. $2 \times 10^{18}$ protons on target were taken.

\section{SERPUKHOV-136}

(Proposed 1978, Approved Apr 1978, Began data-taking 1988;

In progress)

\section{NEUTRINO DETECTOR}

SERPUKHOV - A $A$ Borisov, N I Bozhko, S K Chernichenko, G L Chukin, V N Goryachev, M M Kirsanov, A I Kononov, A S Kozhin, V I Kravtsov, A V Kulikov, A I Mukhin, Y I Salomatin, V A Tumakov, A S Vovenko ( $\sqrt{ }$ Spokesperson)

DUBNA - L S Barabash. Y A Batusov, S A Bunjatov

( $\sqrt{ }$ Spokesperson), O L Klimov, V V Lyukov, Y A Nefedov,

B A Popov, V I Snyatkov, V Y Valuev

Accelerator SERPUKHOV Detector Calorimeter

\section{Reactions}

$$
\begin{array}{lc}
p \text { nucleon } \rightarrow \text { charm } \mathrm{X} & 70 \mathrm{GeV} / c \\
p \text { nucleon } \rightarrow e^{ \pm} \mathrm{X} & " \\
\nu_{\mu} \text { nucleon } \rightarrow \mu^{-} \mathrm{X} & 5-30 \mathrm{GeV} / c \\
\nu_{\mu} \text { nucleon } \rightarrow \mu^{+} \mu^{-} \mathrm{X} & " \\
\nu_{\mu} \text { nucleon } \rightarrow \text { charm X } & " \\
\bar{\nu}_{\mu} \text { nucleon } \rightarrow \mu^{+} \mathrm{X} & " \\
\bar{\nu}_{\mu} \text { nucleon } \rightarrow \mu^{+} \mu^{-} \mathrm{X} & " \\
\bar{\nu}_{\mu} \text { nucleon } \rightarrow \text { charm X } & " \\
\nu_{e} \text { nucleon } \rightarrow e^{ \pm} \mathrm{X} & " \\
\bar{\nu}_{e} \text { nucleon } \rightarrow e^{ \pm} \mathrm{X} & " \\
\text { charm } \rightarrow \mu^{+} \mathrm{X} & - \\
\text { charm } \rightarrow e^{ \pm} \mathrm{X} & --
\end{array}
$$

\section{Particles studied charm}

Brief description Searches for $\nu_{e} \rightarrow \nu_{x}$ oscillation. Running (April 96).

Journal papers $\quad$ YF $30(1979) 702=$ SJNP $30(1979) 362$, YF 33

(1981) $715=$ SJNP 33 (1981) 371, YF 40 (1984) $739=$ S.NP 40

(1984) 475, YF 49 (1989) 172, ZPHY C51 (1991) 341, IJMP A7

(1992) 3835, YF 55 (1992) 2092, PL B279 (1992) 405, PL B295

(1992) 154, PL B302 (1993) 336, YF 57 (1994) $2050=$ PAN 57 (1994) 1974, PL B369 (1996) 39, and ZPHY C70 (1996) 39.

E-mail contact vovenko@mx.ihep.su

\section{SERPUKHOV-145}

(Proposed 1981. Approved 1984, Began data-taking 1987, Completed data-taking 1992)

STUDY OF THE PRODUCTION AND DECAY PROP. ERTIES OF THE CHARMED BARYONS IN NEUTRINO INTERACTIONS WITH THE BUBBLE CHAMBER. SKAT

SERPUKHOV - V V Ammosov ( $\sqrt{ }$ Spokesperson), E N Ardashev, Y V Bardin, A P Bugorsky, N A Chabrov, V I Ermolacv,

V S Fillipov, A A Ivanilov, V I Khleborad, V I Konyushko,

V M Korablev, V A Korotkov, V V Makeev, G Y Mitrofanov,

A G Myagkov, N A Netyaga, A A Sokolov, I L Vasiliev

Accelerator SERPUKHOV Detector HLBC-SKAT

Reactions

$$
\begin{array}{lc}
\nu_{\mu} p \rightarrow \Sigma_{c}(2455)^{++} \mu^{-} & 5-20 \mathrm{GeV} / c \\
\nu_{\mu} p \rightarrow \Sigma_{c}(2530)^{++} \mu^{-} & " \\
\nu_{\mu} n \rightarrow \Lambda_{c}^{+} \mu^{-} & "
\end{array}
$$

Particles studied $\Sigma_{c}(2455)^{++}, \Sigma_{c}(2530)^{++}, \Lambda_{c}^{+}$

Brief description The chamber fill is a light freon-propane mix.

$4 \times 10^{18}$ protons on target were taken.

Journal papers ZETFP 58 (1993) 241.

E-mail contact ammosov@mx.ihep.su

\section{SERPUKHOV-147}

(Proposed 1982, Approved Mar 1982, Began data-taking 1984 Completed data-taking 1991)

STUDY OF REACTIONS WITH STRANGE PARTICLE PRODUCTION IN THE $\pi^{-}$AND $K^{-}$MESON BEAM OF THE IHEP ACCELERATOR

MOSCOW, ITEP -- B P Barkov, B V Bolonkin, I A Erofeev,

O N Erofeeva, V K Grigoriev, A P Grishin, Y V Katinov,

I Y Korolkov, V N Luzin, V V Miller, V N Nozdrachev,

Y P Shkurenko, V V Sokolovsky ( $\sqrt{ }$ Spokesperson), A I Su-

tormin, G D Tikhomirov, V V Vladimirsky

Accelerator SERPUKHOV Detector MIS

\section{Reactions}

$$
\begin{aligned}
& \pi^{-} p \rightarrow n \Lambda \bar{\Lambda} \\
& \pi^{-} p \rightarrow n \Lambda \bar{\Lambda} \pi^{0} \\
& \pi^{-} p \rightarrow p \Lambda \bar{\Lambda} \pi^{-} \\
& \pi^{-} p \rightarrow n 2 K_{S} \\
& \pi^{-} p \rightarrow n K_{S} K_{L} \\
& \pi^{-} p \rightarrow n 2 K_{S} \pi^{0} \\
& \pi^{-} p \rightarrow n K_{S} K_{L} \pi^{0} \\
& \pi^{-} p \rightarrow p 2 K_{S} \pi^{-} \\
& \pi^{-} p \rightarrow p K_{S} K_{L} \pi^{-} \\
& \pi^{-} p \rightarrow n \Sigma^{0} \bar{\Sigma}^{0} \\
& \pi^{-} p \rightarrow \text { glueball X } \\
& \pi^{-} p \rightarrow f_{2}(1720) \times \\
& \pi^{-} p \rightarrow \mathrm{X} C(1480)^{-} \\
& K^{-} p \rightarrow \Lambda \bar{\Lambda} Y^{*}(\text { unspec) } \\
& K^{-} p \rightarrow K_{S} K_{L} Y^{*}(\text { unspec) } \\
& \text { glueball } \rightarrow 2 K_{S} \\
& f_{2}(1720) \rightarrow 2 K_{S} \\
& C(1480)^{-} \rightarrow K_{S} K_{L} \pi^{-}
\end{aligned}
$$

$40 \mathrm{GeV} / c$

$$
\begin{aligned}
& \text { " } \\
& \text { " } \\
& " \\
& " \\
& " \\
& " \\
& " \\
& " \\
& " \\
& " \\
& + \\
& - \\
& -
\end{aligned}
$$

Particles studied $f_{0}(975), a_{0}(980)^{0}, f_{2}(1720)$, glueball,

$C(1480)^{-}, Y^{*}$ (unspec), $f_{2}^{\prime}(1525)$

Brief description Requested 2400-3000 hours. 


\section{SUMMARIES OF SERPUKHOV EXPERIMENTS}

Journal papers YF 43 (1986) 1211, YF 43 (1986) $1487=$ SJNP 43 (1986) 959 , YF 46 (1987) 799, NP B309 (1988) 426, and YF 48 (1988) $1213=\mathrm{SJNP} 48$ (1988) 770.

Related experiments SERPUKHOV-173

E-mail contact sokolovsky@vxitep.itep.ru

\section{SERPUKHOV-152}

(Proposed 1983, Approved Aug 1984, In preparation)

NEUTRINO EXPERIMENT USING A TAGGED NEUTRINO BEAM

SERPUKHOV -. V V Ammosov, V B Anykeyev, A A Bel'kov,

S V Belikov, A P Bugorsky, A Chesnokov, A G Denisov,

S P Denisov ( $\sqrt{ }$ Spokesperson), A Y Dushkin, N N Fedyakin,

A N Galyaev, N A Galyaev, S S Gershtein, Y V Gilitsky,

S N Gurzhiev, V I Kochetkov, V I Kotov, A V Kozelov,

V P Kryuchkov, V I Kurbakov, A A Lebedev, V N Lebedev, V V Lipajev, A Y Maslov, S A Medved, V N Mikhailin, Y V Mikhailov, V A Onuchin, Y M Pishchalnikov, A V Schukin,

I V Shein, A P Soldatov, A A Spiridonov, A P Starkov,

D A Stoyanova, A V Uzunyan

INFN, PISA .. C Cerri, G Gennaro, F Sergiampietri, G Spandre

INFN, FLORENCE - G Conforto, A Marchionni

BERLIN-ZEUTHEN ADW - J Baehr, G Bohm, R Nahnhauer, $S$ Nowak, A Schwind

DUBNA - J Cvach, V K Dodokhov, N G Fadeev, V Genchev,

I A Golutvin, J Hladky, V G Kadykov, V Y Karzhavin,

V S Khabarov, Y T Kiryushin, V G Krivokhizhin, V V Kukhtin,

V N Lysyakov, P K Markov, S Nemecek, A A Popov, D Pose,

A Prokes, P Reimer, S Riman, I A Savin, G I Smirnov,

D A Smolin, J Strachota, G Sultanov, L V Svetov, V $A$ Sviridov,

P Todorov, M Vinde, I Zacek, N I Zamyatin

Accelerator SERPUKHOV Detector Combination

\section{Reactions}

$\nu_{e} e^{-} \rightarrow e^{-} \nu_{e}$

$\nu_{\mu} e^{-} \rightarrow e^{-} \nu_{\mu}$

$\nu_{e}$ nucleon $\rightarrow e^{-} \mathrm{X}$

$\nu_{e}$ nucleon $\rightarrow \nu_{e} \mathrm{X}$

$\nu_{e}$ nuclcon $\rightarrow r^{-} \mathrm{X}$

$\nu_{e}$ nucleon $\rightarrow e^{-} \mu^{+} \mathrm{X}$

$\nu_{\mu}$ nucleon $\rightarrow \mu^{-} \mathrm{X}$

$\nu_{\mu}$ nucleon $\rightarrow \nu_{\mu} \mathrm{X}$

$\nu_{\mu}$ nucleon $\rightarrow \mu^{+} \mu^{-} \mathrm{X}$

$$
<70 \mathrm{GeV}\left(\mathrm{E}_{\mathrm{Iab}}\right)
$$

charmed-meson $\rightarrow \mu^{+} \mathrm{X}$

Particles studied $\nu_{e}, \nu_{\mu}, \tau^{-}$, charmed-meson

Brief description Some of the physics goals are a study of the $\nu_{e}-\nu_{\mu}$ universality and $\nu_{e} \rightarrow \nu_{\mu} \rightarrow \nu_{T}$ oscillations, and a measurement of the charged to neutral currents ratio.

Journal papers YF 52 (1990) 1040.

E-mail contact denisov@mx.ihep.su

\section{SERPUKHOV-157}

(Proposed 1983. Approved Mar 1983, Began data-taking 1986, In progress)

NEW RADIAL-EXITED RESONANCES SEARCH IN DIFFRACTIVE PROCESSES ON NUCLEI WITH DETECTOR MIS-2

DUBNA - M A Ananjeva, V V Antipov, $\mathrm{X}$ I Ivanshin,

I G Kosarev, V A Moiseenko, V A Petrov, Y P Petukhov,

S Y Sychkov, A A 'Tyapkin (V Spokesperson), I M Vasilevsky,

V V Vishnyakov, O A Zaymidoroga

MILAN U - P L Frabetti, P F Manfredi, F Palombo

Accelerator SERPUKHOV Detector MIS-2

Reactions

$$
\pi^{-} \mathrm{Si}-\text {-r } 3 \text { pion } \mathrm{Si}
$$

$40 \mathrm{GeV} / c$ $\pi^{-} \mathrm{Be} \rightarrow 3$ pion $\mathrm{Be}$

$40 \mathrm{GeV} / c$

Particles studied meson

Brief description Uses the modified spectrometer MIS with additional spark chambers. Searches for new radial excitations of $\pi, a_{1}(1260), a_{2}(1320)$, and $\pi_{2}(1670)$ mesons. Requested running time is 720 hours.

Journal papers YF 43 (1986) $917=$ S.JNP 43 (1986) 585, and NC A107 (1994) 2855.

Related experiments FNAL-706, SERPUKHOV-164

E-mail contact tyapkin@lshe19.jinr.dubna.su

\section{SERPUKHOV-159}

(Proposed 1983, Approved May 1986, Began data-taking 1992, In progress)

INVESTIGATION OF CHARMED PARTICLES AND

SEARCH FOR MULTIQUARK STATES USING EXCHARM SPECTROMETER AT THE SERPUKHOV ACCELERATOR

DUBNA - A N Aleev, V P Balandin, I I Evsikov, P Z Hristov, I M Ivanchenko, $Z \mathrm{M}$ Ivanchenko, $\mathrm{M} N$ Kapishin, $\mathrm{N}$ N Karpenko, V D Kekelidze ( $\checkmark$ Spokesperson), I G Kosarev, Z I Kozhenkova. Y A Kozhevnikov, Y A Kretov, N A Kuz'min, A L Lyubimov, D T Madigojin, A S Mestvirishvili, P V Moisenz, A N Morozov, V V Pal'chik, Y K Potrebenikov, T G Progulova, V A Sashin, V E Simonov, A G Skripnichuk, V N Spaskov, G T Tatishvili, A L Tkachev, I P Yudin, O I Yuldashev, M B Yuldasheva, A I Zinchenko

ALMA ATA, PHYS INST $-\Lambda$ A Loktionov

KAZAKH STATE U - G K Potrebenikov

LEBEDEV INST - S P Baranov, M V Belov, V A Kozlov,

$S$ Y Potashev

MINSK, INST NUCL PROBLEMS - A S Kurilin

BUCHAREST, INST PHYS - A Bragadireanu, L Groza,

$M$ Iliesku, T Ponta

SERPUKHOV - A P Bugorski

SOFIYA, INST CHEM 'I'ECH - V Zayachki

SOFIYA, INST NUCL RES - I M Geshkov, S Piperov

TBILISI STATE U - L N Abesalashvili, N S Amaglobeli,

B G Chiladze, M V Kopadze, R A Kvatadze, N L Lomidze,

G I Nikobadze, T G Pitskhelauri

TBILISI, INST PHYS - T S Grigalashvil

Accelerator SERPUKHOV Detector EXCHARM

Reactions

$n$ nucleus $\rightarrow X(3100) X$

$n$ nucleus $\rightarrow X(3250) X$

$n$ nucleus $\rightarrow N \phi(1950) \mathrm{X}$

$n$ nucleus $\rightarrow \ddot{D}^{0} \mathrm{X}$

$n$ nucleus $\rightarrow \Sigma_{c}(2455)^{++} \mathrm{X}$

$n$ nucleus $\rightarrow \Sigma_{c}(2455)^{0} \mathrm{X}$

$n$ nucleus $\rightarrow \Lambda_{c}^{+} \mathrm{X}$

$n$ nucleus $\rightarrow \Xi_{c}^{+} \mathrm{X}$

$n$ nucleus $\rightarrow \Xi_{c}^{0} \mathrm{X}$

$n$ nucleus $\rightarrow \phi \mathrm{X}$

$<70 \mathrm{GeV}\left(\mathrm{E}_{\mathrm{lab}}\right)$

"

Particles studied $X(3100), X(3250), N \phi(1950), \bar{D}^{0}, \Lambda_{c}^{+}$,

$\Sigma_{c}(2455)^{++}, \Sigma_{c}(2455)^{0}, \Xi_{c}^{+}, \Xi_{c}^{0}, \phi$

Brief description Uses the EXCHARM detector.

Journal papers PTE 4 (1995) 8, JINR PAPID COM 3(77) (1996) 5.

E-mail contact kekel@sunse.jinr.dubna.su

\section{SERPUKHOV-164}

(Proposed 1980, Approved May 1986, Began data-taking 1988, Completed data-taking Mar 1996)

INVESTIGATIONS OF THE $\pi^{-} p \rightarrow n \pi^{+} \pi^{-} \pi^{+} \pi^{-}(\gamma$ 's) REACTION AT $40 \mathrm{GeV} / c$ USING THE VERTEX SPECTROMETER 


\section{SUMMARIES OF SERPUKHOV EXPERIMENTS}

SERPUKHOV - D V Amelin, E B Berdnikov, S I Bityukov,

G V Borisov, V A Dorofeev, R I Dzhelyadin, Y P Gouz,

X M Ivanyushenkov, I A Kachacv, A N Karyukhin,

Y A Khokhlov, G A Klyuchnikov, V F Konstantinov,

S V Kopikov, M E Kostrikov, V V Kostyukhin, A A Kriushin,

$M A$ Kulagin, S A Likhoded, V D Matveev, A P Ostankov,

D I Ryabchikov, O V Solovianov, E A Starchenko,

N K Vishnevsky, E A Vlasov, A M Zaitsev ( $\checkmark$ Spokesperson)

TBILISI, INST PIIYS $-\Upsilon \Lambda$ Lomtadze, G G Sekhniaidze,

E G Tskhadadze

Accelerator SERPUKHOV Detector VES

Reactions

$$
\begin{aligned}
& \pi^{-} p \rightarrow n 2 \pi^{+} 2 \pi^{-}\left(\gamma^{\prime} s\right) \\
& \pi^{-} p \rightarrow n 2 \pi^{+} 2 \pi^{-} \\
& \pi^{-} p \rightarrow n 2 \rho^{0} \\
& \pi^{-} p \rightarrow n 2 \eta^{\prime} \\
& \pi^{-} p \rightarrow n f_{1}(1285) \\
& \pi^{-} p \rightarrow n \eta^{\prime} \eta \\
& \pi^{-} p \rightarrow n \omega \eta \\
& \pi^{-} p \rightarrow n \text { meson (mesons) } \\
& \pi^{-} p \rightarrow p \text { meson (mesons) } \\
& \pi^{-} p \rightarrow n \pi^{+} \pi^{-} \eta \\
& \pi^{-} p \rightarrow p \pi^{-} \eta \eta \\
& \pi^{-} p \rightarrow n \omega \omega^{-} \\
& \pi^{-} p \rightarrow p K^{+} K^{-} \pi^{-} \\
& K^{-} p \rightarrow p K^{-} \pi^{+} \pi^{-} \\
& K^{-} p \rightarrow p K^{-} \pi^{+} \pi^{-} \pi^{0}
\end{aligned}
$$

Particles studied $\rho^{0}, \eta, \eta^{\prime}, \omega, f_{1}(1285), \pi(1770)$, exotic, glueball, meson

Brief description Uses VES (VErtex Spectrometer) - a wide aperture magnetic spectrometer combined with a lead glass gamma detector and Cerenkov identifiers. In addition to the reactions listed above, also studies decays of the mesons produced in the reactions.

Journal papers PL B268 (1991) 137, ZPHY C54 (1992) 235, ZPHY C54 (1992) 367, YF 55 (1992) 2460, ZPHY C57 (1992) 13, PL B313 (1993) 276, PL B337 (1994) 219, ZPHY C66 (1995) $71, \mathrm{PL} \mathrm{B356}$ (1995) 595, ZPHY C80 (1996) 71 , and YF 59 (1996) 1021.

Related experiments BNL-852, SERPUKHOV-163

E-mail contact zaitsev@mx.ihep.su

WWW Home-page http://dxbec.ihep.su/

\section{SERPUKHOV-166}

(Proposed 1987, Approved 1987, Began data-taking 1987)

\section{STUDY OF RARE DECAYS WITH THE ISTRA-M} DETECTOR

MOSCOW, INR - V N Bolotov (Spokesperson), E N Gushchin $\mathrm{V} V$ Isakov, O V Karavichev, V A Lebedev, V N Marin, Y V Musienko, A $A$ Poblaguev, V E Postoev, G N Semenuk, $S$ A Volkov

SERPUKHOV - V F Konstantinov

DUBNA - G Kalmar, A Z Kitikyan, E V Komissarov, V S Kurbatov, V Z Serdyuk, V V Sidorov, A D Volkov, B Z Zalikhanov

Accelerator SERPUKHOV Detector ISTRA-M

$\begin{array}{lc}\frac{\text { Reactions }}{K^{-} \rightarrow \pi^{-} \nu_{e} \bar{\nu}_{e}} & 25 \mathrm{GeV} / c \\ K^{-} \rightarrow \pi^{-} \nu_{\mu} \bar{\nu}_{\mu} & " \\ K^{-} \rightarrow e^{-} \bar{\nu}_{e} \gamma & " \\ K^{-} \rightarrow \pi^{-} e^{-} e^{+} & " \\ K^{-} \rightarrow \pi^{-} \mu^{-} \mu^{+} & "\end{array}$

Particles studied $\pi^{-}, K^{-}$

E-mail contact bolotov@inr.msk.su

\section{SERPUKHOV-167}

(Proposed 1975, Approved 1987, Began data-taking 1987, In progress)

\section{STUDY OF RARE KAON DECAYS}

SERPUKHOV - A M Blick, V N Kolosov, V M Kutjin, V N Shelikhov

DUBNA - V Y Batusov, Y A Budagov, I E Chirikov-Zorin, Y I Davydov, V B Flyagin ( $\sqrt{ }$ Spokesperson), V V Glagolev, A V Kolomyichenko, Y F Lomakin, S N Malyukov, O E Pukhov, V I Romanovsky, N A Rusakovich, N L Rusakovich, A A Semenov, A N Shaljugin, A S Soloviev, V B Vinogradov, A G Volodko

TBILISI STA'I'E U - G A Chlachidze, I A Minashvili SOFIYA U - A B Jordanov, L Litov, G V Velev MINSK, INST PHYS - Y A Kulchitsky, A S Kurilin Accelerator SERPUKHOV Detector HYPERON-II Reactions

$\begin{array}{lc}K^{+} \rightarrow \pi^{+} 2 \pi^{0} & 10 \mathrm{GeV} / c \\ K^{+} \rightarrow \pi^{+} \pi^{0} \gamma & " \\ K^{+} \rightarrow \pi^{+} 2 \gamma & " \\ K^{+} \rightarrow \pi^{0} e^{+} \nu_{e} & " \\ K^{+} \rightarrow 2 \pi^{0} e^{+} \nu_{e} & " \\ K^{+} \rightarrow \pi^{0} e^{+} \nu_{e} \gamma & " \\ K_{S} \rightarrow e^{-} e^{+} & " \\ K_{S} \rightarrow 2 \gamma & " \\ K_{S} \rightarrow e^{-} e^{+} \gamma & " \\ K_{S} \rightarrow \pi^{0} e^{-} e^{+} & "\end{array}$

Particles studied $K^{+}, K_{S}$

Brief description The setup consists of a pair of one-arm spectrometers: onc to measure the beam momentum, and the second, a large aperture spectrometer, to register the secondary charged particles momenta. All coordinates in the spectrometers are measured by proportional chambers (about 10,000 channels). Two large lead glass multichannel ( 850 channels) hodoscopic spectrometers are used to detect $\gamma$ energies. Taking data (July 96).

Journal papers PL B259 (1991) 225, PL B334 (1994) 234, and PTE 3 (1994) 13.

Related experiments SERPUKHOV-166, BNL-791, BNL-845 E-mail contact flyagin@main1.jinr.dubna.su

\section{SERPUKHOV-169}

\begin{tabular}{|c|c|}
\hline$\underset{\substack{p \text { nucleon } \\
\text { nucleon }}}{\rightarrow} \mathrm{DD}<p K^{+} K^{-}>$ & $70 \mathrm{GeV}\left(\mathrm{E}_{\text {lab }}\right)$ \\
\hline$p$ nucleon $\rightarrow \mathrm{DD}\langle p \phi>$ nucleon & $"$ \\
\hline$p$ nucleon $\rightarrow \mathrm{DD}<\Lambda K^{+}>$nucleon & " \\
\hline$p$ nucleon $\rightarrow \mathrm{DD}<\Lambda\left(1405 S_{01}\right)$ & $"$ \\
\hline $\begin{aligned} K^{+} & >\text {nucleon } \\
p \text { nucleon } & \rightarrow \mathrm{DD}<\Lambda\left(1520 D_{03}\right) \\
K^{+} & >\text {nucleon }\end{aligned}$ & $"$ \\
\hline$p$ nucleon $\rightarrow \mathrm{DD}<\Sigma^{0} K^{+}>$nucleon & " \\
\hline
\end{tabular}

(Proposed 1977, Approved Jul 1977, Began data-taking 1985 Completed data-taking 1993)

\section{INVESTIGATIONS OF HADRONIC SPECTROSCOPY} WITH THE DETECTOR SPHINX

SERPUKHOV - S V Golovkin, A P Kozhevnikov, V P Kubarovsky, A I Kulyavtsev, V F Kurshetsov, A E Kushnerenko, L G Landsberg ( $\checkmark$ Spokesperson), V V Molchanov, V A Mukhin, IN Nikitin, A V Skleznev, V I Solyanik, D V Vavilov, V A Viktorov

MOSCOW, I'TEP - M Y Balatz, G B Dzyubenko, G K Kliger,

V Z Kolganov, V S Lakaev, G S Lomkatzi, A F Nilov,

V T Smolyankin, V E Vishnyakov

Accelerator SERPUKHOV Detector SPHINX

Reactions 


\section{SUMMARIES OF SERPUKHOV EXPERIMENTS}

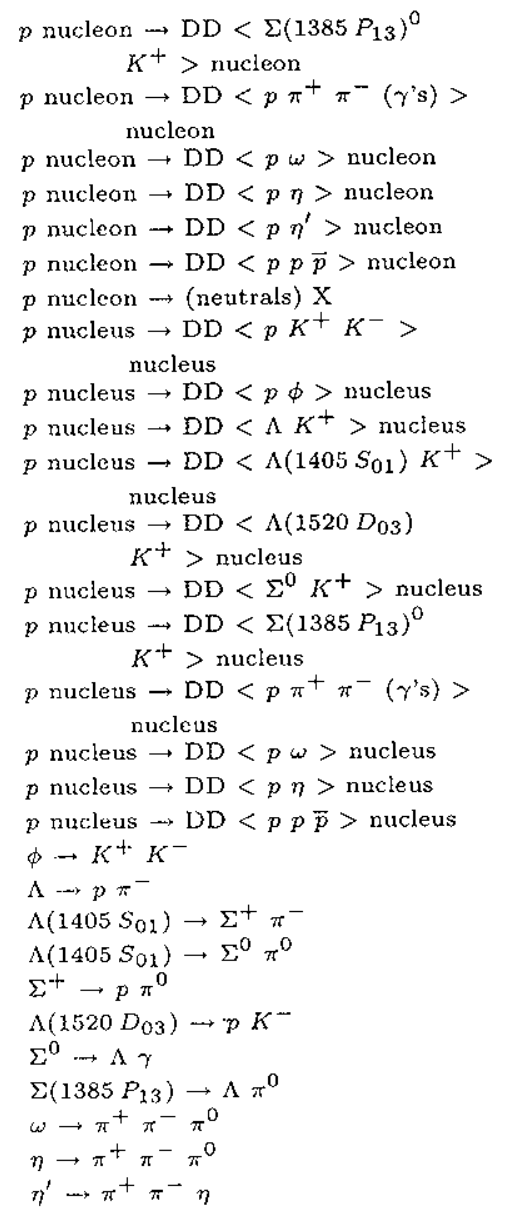

$70 \mathrm{GeV}\left(\mathrm{E}_{\mathrm{lab}}\right)$

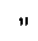

"

1

$"$

It

11

$"$

$"$

11

$"$

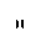

"
"

Particles studied baryon, $N \phi(1950), \Sigma(3170 \mathrm{~B})^{+}$

Brief description Studies baryon diffractive production, coherent reactions on nuclei, and searches for exotic baryons including pentaquark cryptoexotic baryon resonances with hidden strangeness in the mass region up to $4.5 \mathrm{GeV} / \mathrm{c}^{2}$. Uses $B e$ and $C$ as nuclear targets. SPHINX consists of a wide aperture magnetic spectrometer with proportional and drift chambers working in combination with a multichannel gamma spectrometer and a system of Cerenkov detectors for the identification of charged secondary particles. Data analysis completed.

Journal papers $\quad$ YF 52 (1990) 494, YF 57 (1994) 47, YF 57 (1994) 241, YF 57 (1994) 253, ZPHY C61 (1994) 223, ZPHY C61 (1994) 399, NC A107 (1994) 2441, YF 57 (1994) 1376, YF 57 (1994) 2030, YF 57 (1994) 2042, UFN 164 (1994) 1129, YF 58 (1995) 1426, and ZPHY C68 (1995) 585.

Related experiments SERPUKHOV-120, SERPUKHOV-178

E-mail contact $\operatorname{lgl} @ \mathrm{mx}$.ihep.su

\section{SERPUKHOV-172}

(Proposed 1988, Approved May 1988, Began data-taking Dec 1991, In progress)

STUDY OF MESONS WITH AN ENHANCED GLUON COMPONENT (GLUEBALLS INCLUDED) AND MESONS WITH HIGH SPINS USING THE MULTIPHOTON $4 \pi$ SPECTROMETER

SERPUKHOV - A V Dolgopotov, S V Donskov, A V Inyakin,

G V Khaustov, A A Kondashov, A K Konoplyannikov,

A V Kulik, A A Lednev, V A Lishin, Y M Melnik,

S A Polovnikov, V A Polyakov, Y D Prokoshkin

( $\sqrt{ }$ Spokesperson), S A Sadovsky, V D Samoylenko, P M Shagin, A V Shtannikov, A V Singovsky, E A Sobol, V P Sugonyaev TBILISI, INST PHYS - A K Djavrishvili, T A Lomtadze,

G G Sekhniaidze, E G Tskhadadze

LOS ALAMOS -. D Alde, E A Knapp, T Lopez

BRUSSELS U, IISN - F Binon, J P Stroot

ANNECY - J P Peigneux, M Poulet

KEK - S Inaba, M Kobayashi, T Tsuru

MIYAZAKI U - T Nakamura, K Takamatsu Accelerator SERPUKHOV Detector GAMS-4PI

\section{Reactions}

$$
\begin{aligned}
& \pi^{-} p \rightarrow n 2 \pi^{0} \\
& \pi^{-} p \rightarrow n 2 \eta \\
& \pi^{-} p \rightarrow n \eta^{\prime} \eta \\
& \pi^{-} p \rightarrow n \eta \pi^{0} \\
& \pi^{-} p \rightarrow n \eta 2 \pi^{0} \\
& \pi^{-} p \rightarrow n 2 \omega \\
& \pi^{-} p \rightarrow n K^{0} \bar{K}^{0} \\
& \pi^{-} p \rightarrow n \text { 2meson }^{0} \\
& \pi^{-} p \rightarrow \operatorname{meson}^{0} \mathrm{X} \\
& \pi^{-} p \rightarrow \text { giveball } \mathrm{X} \\
& \pi^{-} p \rightarrow J / \psi(1 S) \mathrm{X} \\
& \pi^{-} p \rightarrow \psi(2 S) \mathrm{X} \\
& \pi^{-} p \rightarrow \eta_{c}(1 S) \mathrm{X} \text {. } \\
& \pi^{-} p \rightarrow \chi_{c} \text { (unspec) } \mathrm{X} \\
& K^{-} p \rightarrow \text { meson }^{0} \mathrm{X} \\
& \text { glueball } \rightarrow 4 \pi^{\circ} \\
& \text { glucball } \rightarrow 2 \eta \\
& \text { glueball } \rightarrow \eta^{\prime} \eta \\
& \text { glueball } \rightarrow 2 \eta^{\prime} \\
& \text { meson }{ }^{0} \rightarrow 2 \pi^{\circ} \\
& \text { meson }^{0} \rightarrow 2 \eta \\
& \operatorname{meson}^{0} \rightarrow \eta^{\prime} \pi^{0} \\
& \eta_{c}(1 S) \rightarrow \eta 2 \pi^{0} \\
& \pi^{0} \rightarrow 2 \gamma \\
& \eta \rightarrow 2 \gamma
\end{aligned}
$$

$32 \mathrm{GeV} / \mathrm{c}$

$$
\begin{aligned}
& " 1 \\
& " \\
& " \\
& " \\
& " \\
& " \\
& " \\
& " \\
& " \\
& " \\
& " \\
& " \\
& " \\
& -\cdots \\
& - \\
& \hline- \\
& \hline- \\
& -\ldots \\
& - \\
& - \\
& -
\end{aligned}
$$$$
"
$$$$
+
$$$$
11
$$

Particles studied glucball, meson ${ }^{0}$

Brief description Data taking and data analysis are in progress (May 96).

Journal papers NIM A268 (1988) 112, NIM A276 (1989) 652, PTE 1 (1990) 68, PTE 2 (1990) 90, PTE 5 (1991) 55, and NIM A302 (1991) 443 .

Related experiments BNL-852

E-mail contact prokoshkin@mx.ihep.su

\section{SERPUKHOV-173}

(Proposed 1991, Approved 1992, Began data-taking 1992,

In progress)

STUDY OF STRANGE PARTICLE RESONANT STATES USING HADRON BEAMS WITH MOMENTA OF 40-70 $\mathrm{GeV} / \mathrm{AT}$ THE IHEP ACCELERATOR

MOSCOW, I'IEP - B P Barkov, I A Erofeev, O N Erofeeva,

V K Grigoriev, A P Grishin, Y V Katinov, I Y Korolkov,

$V$ I Lisin, V N Luzin, V N Nozdrachev ( $V$ Spokesperson),

Y P Shkurenko, V V Sokolovsky, G D Tikhomirov,

V V Vladimirsky

Accelerator SERPUKHOV Detector MIS

$$
\begin{aligned}
& \frac{\text { Reactions }}{\pi^{-} p \rightarrow n 2 K_{S}} \\
& \pi^{-} p \rightarrow n 2 K_{S} \pi^{0} \\
& \pi^{-} p \rightarrow n K_{S} K_{L} \pi^{+} \pi^{-} \\
& \pi^{-} p \rightarrow n K_{S} K_{L} \pi^{0} \\
& \pi^{-} p \rightarrow K_{S} \pi^{+} \pi^{-} Y^{*} \text { (unspcc) } \\
& \pi^{-} p \rightarrow p K_{S} K_{L} \pi^{-} \\
& \pi^{-} p \rightarrow n \Lambda \Lambda
\end{aligned}
$$

$40 \mathrm{GeV} / \mathrm{c}$

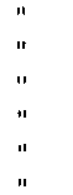




\section{SUMMARIES OF SERPUKHOV EXPERIMENTS}

$$
\begin{aligned}
& K^{-} p \rightarrow 2 K_{S} Y^{*} \text { (unspec) } \\
& K^{-} p \rightarrow n K_{S} \pi^{+} \pi^{-} \\
& K^{-} p \rightarrow \Lambda \bar{\Lambda} Y^{*} \text { (unspec) }
\end{aligned}
$$

Particles studied $C(1480)^{-}, C(1480)^{0}, \rho(1700)^{0}, X(3100)$, $\phi(1680), \phi_{3}(1850), f_{2}(1720), f_{2}(1810), f_{2}(2010), f_{4}(2050)$, $f_{4}(2220), K_{2}^{*}(1430)^{-}$

Brief description Extends an carlier $K_{S} K_{S}$ and $\Lambda \bar{\Lambda}$ final states study (SERPUKHOV-147) to the range of masses between 1.8 and $2.5 \mathrm{GeV}$. Investigates the $K_{S} K_{L}$ system using $\pi^{-}$and $K^{-}$beams with the momentum of $40 \mathrm{GeV} / c$. Studies baryonantibaryon and $\phi \phi$ states. Searches for $C(1480)$ mesons by detecting $K^{0} K^{*}$ pairs. Uses two charged-particle triggers. Requested 2100 hours.

Journal papers PTE 3 (1994) 43, YF 58 (1995) $50=$ PAN 58 (1995) 46, and YF 58 (1995) 1628.

Related experiments SERPUKHOV-147

E-mail contact nozdrachev@mx.ihep.su, nozdrachev@vxitep.itep.ru

\section{SERPUKHOV-177}

(Proposed 1990, Approved Jul 1993, In preparation)

\section{MEASUREMENT OF THE MASS OF THE $\Sigma^{-}$} HYPERON

ST PETERSBURG, INP - A S Denisov, O L Fedin, M P Guriyev, X M Ivanov ( $\checkmark$ Spokesperson), L P Lapina, P M Levchenko, A A Petrunin, Y P Platonov, V M Suvorov, A V Zhelamkov Accelerator SERPUKHOV Detector QUARTZ

\section{Reactions}

$p \mathrm{C} \rightarrow \Sigma^{-} \mathrm{X}$

$p \mathrm{Mg} \rightarrow \Sigma^{-} \mathrm{X}$

$70 \mathrm{GeV} / \mathrm{c}$

$\mathrm{Cu} \rightarrow \Sigma^{-} \mathrm{X}$

$"$

$p \mathrm{~Pb} \rightarrow \Sigma^{-} \mathrm{X}$

Particles studied $\Sigma^{-}$

Brief description Approved for 360 hours. QUARTZ is a crystal diffraction spectrometer for hadronic $X$-rays with a semiconductor detector. Waiting for the run time (July 96).

E-mail contact yumi@hep486.pnpi.spb.ru

\section{SERPUKHOV-178}

(Proposed 1994, Approved 1994, In preparation)

\section{SEARCH FOR EXOTIC BARYON STATES WITH THE} MODIFIED DETECTOR SPHINX

SERPUKHOV - S A Akimenko, Y M Antipov, V A Batarin,

V A Bezzubov, A A Derevshchikov, S V Erin, O V Eroshin,

$S$ V Golovkin, Y P Gorin, V N Govorun, A N Isaev,

V A Kachanov, Y D Karpekov, A S Konstantinov,

A P Kozhevnikov, V P Kubarovsky, V F Kurshetsov,

L G Landsberg ( $\checkmark$ Spokcsperson), M Y Matveev,

V A Medovikov, V V Molchanov, V A Mukhin, I N Nikitin,

O V Orel, A I Pavlinov, S V Petrenko, V S Petrov,

A I Petrukhin, P A Semenov, V A Senko, M M Soldatov,

V L Solovianov, V I Solyanik, A N Sytin, V S Vanicv,

D V Vavilov, V A Viktorov, S A Zimin

MOSCOW, ITEP - G K Kliger, V Z Kolganov, G S Lomkatzi,

A F Nilov, V T Smolyankin

Accelerator SERPUKHOV Detector SPHINX

Reactions

$$
\begin{array}{cc}
p \text { nucleon } \rightarrow \mathrm{DD}<p K^{+} K^{-}> & 70 \mathrm{GeV}\left(\mathrm{E}_{\mathrm{lab}}\right) \\
\text { nucleon } & " \\
p \text { nucleon } \rightarrow \mathrm{DD}<p \phi>\text { nucleon } & " \\
p \text { nucleon } \rightarrow \mathrm{DD}<\Lambda K^{+}>\text {nucleon } & " \\
p \text { nucleon } \rightarrow \mathrm{DD}<\Lambda\left(1405 S_{01}\right) & \\
K^{+} & >\text {nucleon } \\
p \text { nucleon } \rightarrow \mathrm{DD}<\Lambda\left(1520 D_{03}\right) & " \\
K^{+}>\text {nucleon } &
\end{array}
$$

$$
\begin{aligned}
& p \text { nucleon } \rightarrow \mathrm{DD}<\Sigma^{0} K^{+}>\text {nucleon } \\
& p \text { nucleon } \rightarrow \mathrm{DD}<\Sigma\left(1385 P\left(P_{13}\right)^{\circ}\right. \\
& K^{+}>\text {nucleon } \\
& p \text { nucleon } \rightarrow \mathrm{DD}<p \pi^{+} \pi^{-}\left(\gamma^{\prime} \mathrm{s}\right)> \\
& \text { nucleon } \\
& p \text { nucleon } \rightarrow \mathrm{DD}<p \omega>\text { nucleon } \\
& p \text { nucleon } \rightarrow \mathrm{DD}\langle p \eta>\text { nucleon } \\
& p \text { nucleon } \rightarrow \mathrm{DD}<p \eta^{\prime}>\text { nucleon } \\
& p \text { nucleon } \rightarrow \mathrm{DD}<p p \vec{p}>\text { nucleon } \\
& p \text { nucleus } \rightarrow \mathrm{DD}<p K^{+} K^{-}> \\
& \text {nucleus } \\
& p \text { nucleus } \rightarrow \mathrm{DD}\langle p \phi\rangle \text { nucleus } \\
& p \text { nucleus } \rightarrow \mathrm{DD}<\Lambda K^{+}>\text {nucleus } \\
& p \text { nucleus } \rightarrow \mathrm{DD}<\Lambda\left(1405 S_{01}\right) K^{+}> \\
& \text {nucleus } \\
& p \text { nucleus } \rightarrow \mathrm{DD}<\Lambda\left(1520 D_{03}\right) \\
& \mathrm{K}^{+}>\text {nucleus } \\
& p \text { nucleus } \rightarrow \mathrm{DD}<\Sigma^{0} K^{+}>\text {nucleus } \\
& p \text { nucleus } \rightarrow \text { DD }<\Sigma\left(1385 P_{13}\right)^{0} \\
& K^{+}>\text {nucleus } \\
& p \text { mucleus } \rightarrow \mathrm{DD}<p \pi^{+} \pi^{-}\left(\gamma^{\prime} \mathrm{s}\right)> \\
& \text { nucleus } \\
& p \text { nucleus } \rightarrow \mathrm{DD}<p \omega>\text { nucleus } \\
& p \text { nucleus } \rightarrow \mathrm{DD}<p \eta>\text { nucleus } \\
& p \text { nucleus } \rightarrow \mathrm{DD}\langle p p \bar{p}>\text { nucleus } \\
& \phi \rightarrow K^{+} K^{-} \\
& \Lambda \rightarrow p \pi^{-} \\
& \Lambda\left(1405 S_{01}\right) \rightarrow \Sigma^{+} \pi^{-} \\
& \Lambda\left(1405 S_{01}\right) \rightarrow \Sigma^{0} \pi^{0} \\
& \Sigma^{+} \rightarrow p \pi^{0} \\
& \Lambda\left(1520 \mathrm{D}_{03}\right) \rightarrow p K^{-} \\
& \Sigma^{0} \rightarrow \Lambda \gamma \\
& \Sigma\left(1385 P_{13}\right) \rightarrow \Lambda \pi^{0} \\
& \omega \rightarrow \pi^{+} \pi^{-} \pi^{0} \\
& \eta \rightarrow \pi^{+} \pi^{-} \pi^{0} \\
& \eta^{\prime} \rightarrow \pi^{+} \pi^{-} \eta
\end{aligned}
$$

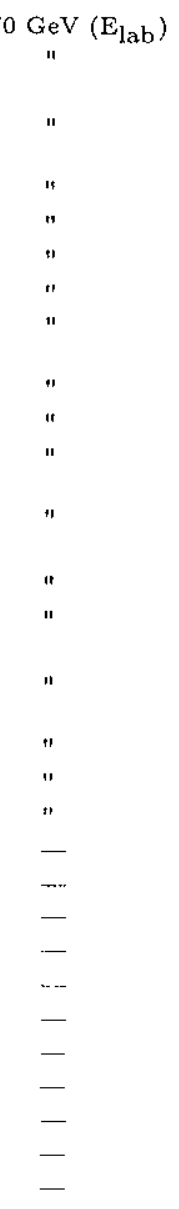

\section{SERPUKHOV-UNK-001}

(Proposed 1988, Approved Apr 1988, In preparation)

STUDY OF SPIN EFFECTS AT 100 TO 600 GeV USING AN INTERNAL JET TARGET AT UNK

SERPUKHOV -- G A Alekseev, Y I Arestov, N I Belikov. V V Borog, B V Chujko, V S Datsko, A M Davidenko, A A Derevshchikov, A M Gorin, O A Grachev, V N Grishin,

V A Kachanov, Y V Kharlov, V Y Khodyrev, V G Lapshin,

V M Leontiev, I V Manujlov, Y A Matulenko, V A Medvedev, Y M Melnik, A P Meschanin, N G Minaev, V V Mochalov, A I Mysnik, S B Nurushev, D I Patalakha, A M Pavlinov,

V A Polyakov, A F Prudkoglyad, V I Rykalin, V V Rykalin,

V L Rykov, V L Solovianov ( $\checkmark$ Spokesperson), L F Soloviev,

$S M$ Troshin, O D Tsai, M N Ukhanov, A N Vasiliev,

A E Yakutin, $S$ V Yerin

DUBNA - L S Barabash, S I Bilenkaya, N S Borisov,

V A Budilov, V V Fimushkin, M Finger, N V Gorbunov, 


\section{SUMMARIES OF SERPUKHOV EXPERIMENTS}

N L Gorshkova, V A Kalinnikov, A G Karev, B A Khachaturov, V S Kiselev, B Z Kopeliovich, M I Kulikov, R H Kutuev, E A Ladygin, A B Levkovich, M Y Liburg, V N Matafonov, A B Neganov, V A Nikitin, P V Nomokonov, V P Obudkovsky, Y K Pilipenko, I L Pisarev, Y A Pliss, A A Popov,

I K Potashnikova, M G Shafranova, V V Shutov, V I Snyatkov, $Y$ A Usov, A I Valevich, V $P$ Yershov, I V Zhigulin,

N K Zhydkov, R Y Zulkarneev

TBILISI STATE U - N S Amaglobeli, Y S Bagaturia,

B G Chiladze, G A Dzhambazishvili, L N Glonty,

G G Macharashvili, $\Lambda$ I Ocherashvili, R M Sakandelidze,

T M Sakhelashvili

MOSCOW STATE U - L I Belzer, A I Demianov,

A M Gribushkin, N A Kruglov, A S Proskuryakov,

L I Sarycheva, N B Sinev, A A Yershov

MICHIGAN U - L V Alexeeva, V A Anferov, B B Blinov,

J A Bywater, C M Chu, D G Crabb, D B Crandell,

Y S Derbenev, S E Gladycheva, S-Q Hu, S V Koutin,

A D Krisch ( $\checkmark$ Spokesperson), A M T Lin, T J Liu,

V G Luppov, D C Peaslee, R A Phelps, L G Ratner,

R S Raymond, M A Skalsey, J A Stewart, S M Varzar,

V K Wong

MIT - G R Court, D Kleppner

Accelerator SERPUKHOV-UNK Detector NEPTUN

\section{Reactions Polarized target}

$p p \rightarrow p p$

$p p \rightarrow \gamma \mathrm{X}$

$p p \rightarrow e^{-} e^{+} \mathrm{X}$

$p p \rightarrow \mu^{-*} \mu^{+} \mathrm{X}$

$p p \rightarrow$ pion $\mathrm{X}$

$p p \rightarrow K^{ \pm} \mathrm{X}$

$p p \rightarrow \eta \mathrm{X}$

$p p \rightarrow \eta^{\prime} \mathrm{X}$

$p p \rightarrow \omega \mathrm{X}$

$p p \rightarrow f_{2}(1270) \mathrm{X}$

$p p \rightarrow$ jet $\mathrm{X}$

$p p \rightarrow \gamma$ jet $\mathrm{X}$

$p p \rightarrow \Lambda \mathrm{X}$

$p p \rightarrow \bar{\Lambda} \mathrm{X}$

$p p \rightarrow p X$

$p p \rightarrow \bar{p} \mathrm{X}$

$p p \rightarrow$ hyperon $\mathrm{X}$

$p p \rightarrow \Sigma^{+} \mathrm{X}$

$p p \rightarrow \Sigma^{-} \mathrm{X}$

$p p \rightarrow \Xi^{-} \mathrm{X}$

$p p \rightarrow \Lambda_{c}^{+} \mathrm{X}$

$p$ nucleus $\rightarrow \gamma \mathrm{X}$

$p$ nucleus $\rightarrow e^{-} e^{+} \mathrm{X}$

$p$ nucleus $\rightarrow \mu^{-} \mu^{+} \mathrm{X}$

$p$ nucleus $\rightarrow$ pion $\mathrm{X}$

$p$ nucleus $\rightarrow K^{ \pm} \mathrm{X}$

$p$ nucleus $\rightarrow \eta \mathrm{X}$

$p$ nucleus $\rightarrow \eta^{\prime} \mathrm{X}$

$p$ nucleus $\rightarrow \omega \mathrm{X}$

$p$ nucleus $\rightarrow f_{2}(1270) \mathrm{X}$

$p$ nucleus $\rightarrow$ jet $\mathrm{X}$

$p$ nucleus $\rightarrow \gamma$ jet $X$

$p$ nuclcus $\cdots \Lambda \mathrm{X}$

$p$ nucleus $\rightarrow \bar{\Lambda} \mathrm{X}$

$p$ nucleus $\rightarrow p \mathrm{X}$

$p$ nucleus $\rightarrow \vec{p} \mathrm{X}$

$p$ nucleus $\rightarrow$ hyperon $\mathrm{X}$

$p$ nucleus $\rightarrow \Sigma^{+} \mathrm{X}$

$p$ nucleus $\rightarrow \Xi^{-} \mathrm{X}$

$p$ nucleus $\rightarrow \Lambda_{c}^{+} \mathrm{X}$

Brief description Studies spin effects when the $600 \mathrm{GeV}$ protons

in the UNK rings collide with a spin-polarized ultra-cold atomic hydrogen internal jet target. Five different spectrometers will observe spin phenomena in various hadron-hadron reactions at small, medium, and large transverse momenta. Journal papers PTE 3 (1991) 52, and P'TE 4 (1991) 57.

Related experiments BNL-794

E-mail contact solovianov@mx.ihep.su, krisch@miphys.physics.lsa.umich.edu

\section{Other Serpukhov Experiments}

Eisted here are some other Serpukhov experiments of interest to the particle physics community. Find more details about these projects online, in the SLAC's EXPERIMENTS database (see p. 3), or contact the spokespersons.

SERPUKHOV-149 (Completed data-taking 1996)

STUDY OF ASYMMETRY IN INCLUSIVE REACTIONS $\pi^{-} \vec{p} \rightarrow \pi^{0} X$ AT $40 \mathrm{GeV} / c$ AND $p \vec{p} \rightarrow \pi^{0} X$ AT $70 \mathrm{GeV} / c$ Spokesperson: A.N. Vasiliev [Serpukhov, IFVE]

E-mail contact vasiliev@mx.ihep.su

SERPUKHOV-155 (Completed data-taking 1992)

SINGLE AND PAIR HADRON PRODUCTION WITH LARGE MOMENTUM TRANSFER IN PROTON AND $\pi^{-}$MESON BEAMS

Spokesperson: V.X. Kryshkin [Serpukhov, IFVE]

E-mail contact kryshkin@mx.ihep.su

SERPUKHOV-161 ('Taking data)

STUDY OF CHARMED PARTICLE PRODUCTION AT IHEP ACCELERATOR ENERGIES

Spokespersons: A.M. Moiseev [Serpukhov, IFVE], P.F. Ermolov [Moscow State U.], I.V. Boguslavsky [Dubna, JINR]

E-mail contact moiseev@mx.ihep.su, ermolov@sgi.npi.msu.su, boguslav@!he08.jinr.dubna.su

SERPUKHOV-171 (Taking data)

DETERMINATION OF ENERGY DEPOSITION IN

THICK TARGETS FROM CONSTRUCTION MATERIALS EXPOSED TO PROTONS WITH KINETIC ENERGIES OF 0.8-1.2 GeV/c

Spokesperson: V.I. Belyakov-Bodin [Moscow, ITEP]

$\underline{E \text {-mail contact }}$ belyakov... b@vxitep.itep.ru

SERPUKHOV-174 (Completed data-taking 1991)

PHYSICS OF RELATIVISTIC DIMESON ATOMS

Spokesperson: L.L. Nemenov [Dubna, JINR]

E-mail contact nemenov@nusun.jinr.dubna.su

SERPUKHOV-175 (Taking data)

SINGLE AND PAIR HADRON PRODUCTION WITH

LARGE MOMENTUM TRANSFER IN POLARIZED PROTON BEAM

Spokesperson: V.I. Kryshkin [Serpukhov, IFVE]

E-mail contact kryshkin@mx.ihep.su 


\section{SUMMARIES OF SLAC EXPERIMENTS}

\section{Selected SLAC Experiments}

\section{SLAC-E-142}

(Proposed Oct 1989, Approved May 1990, Began data-taking Nov 1992, Completed data-taking Dec 1992)

MEASUREMENT OF THE NEUTRON SPIN DEPENDENT STRUCTURE FUNCTION

AMERICAN U - R G Arnold, P E Bosted, J Dunne, C E Keppel,

S E Rock, M Spengos, Z M Szalata, J L White

BONN U - W Meyer

CLERMONT-FERRAND U - V Breton, H Fonvieille

HARVARD U - A K Thompson

LBL .. G Shapiro

LIVERMORE - P L Anthony, F Dietrich, K van Bibber

MICHIGAN U - T E Chupp

PRINCETON U - G Cates, H L Middleton, N Newbury

SACLAY -- H Borel, R Lombard-Nelsen, J Marroncle,

J Morgenstern, F M Staley, Y D Terrien

SLAC - R A Gcarhart, E W Hughes ( $\checkmark$ Spokesperson),

T Maruyama, G M Petratos, R Pitthan, L S Rochester,

$S$ HY Rokni, M B Woods, C C Young

S'CANFORD U -- D M Kawall, S Kuhn, Z E Meziani

SYRACUSE U - R Holmes, P A Souder, $J$ Xu

WISCONSIN U - H Band, I R Johnson, R A Mair, R Prepost,

G H Zapalac

Accelerator SLAC Detector Double-arm spectrometer

Reactions Polarized beam and target

$e^{-3} \mathrm{He} \quad 22.66 \mathrm{GeV} / c\left(\mathrm{P}_{\mathrm{lab}}\right)$

Particles studied $n$

Brief description Studics a polarized electron beam scattering

off a polarized ${ }^{3} \mathrm{He}$ gas target. The scattered electrons are detected by a two-arm fixed spectrometer. Tests the Bjorken polarization sum rule and nucleon spin models. Run for 400 hours. Data analysis in progress (July 96 ).

Journal papers PRL 71 (1993) 959, and NC 107A (1994) 2197.

Related experiments SLAC-E-154

E-mail contact emlyn@slac.stanford.edu

\section{SLAC-E-143}

(Proposed Nov 1991, Approved Dec 1991, Began data-taking Nov 1993, Completed data-taking Feb 1994)

MEASUREMENTS OF THE NUCLEON SPIN STRUCTURE IN END STATION A AT SLAC

E143 COLLABORATION

A.MERICAN U - R G Arnold ( $\checkmark$ Spokesperson), P E Bosted,

J Dunne, J Fellbaum, D Reyna, S E Rock, M Spengos,

Z M Szalata, J L White

BASEL U - A Feltham, I Sick, P Steiner, B Zihlmann

CLERMONT-FERRAND U -. V Breton, C Comptour,

H Fonvieille, Y Roblin

CEBAF - J Gomez, J H Mitchell

DAPNIA, SACLAY - II Borel, P Grenier, R Lombard-Nelsen,

I Marroncle, J Morgenstern, F M Staley, Y D Terrien

LIVERMORE - F Dietrich

MASSACHUSETTS U, AMHERST - J Bauer, J Button-Shafer

MICHIGAN U - T E Chupp, K P Coulter, T B Smith

OLD DOMINION U - C E Hyde-Wright, A Klein, S Kuhn,

B Rauc

PENN U - R Antonov, K A Griffioen, P Raines

SLAC - P L Anthony, 3 Clendenin, M Daoudi, H Dutz,

$R$ Erbacher, $R A$ Gearhart, $E$ W Hughes, ' $T$ Maruyama,

W Meyer, G M G Petratos, R Pitthan, C Prescott,

L S Rochester, S J St Lorant, L M Stuart, H Tang, $T$ Usher.

D R Walz, K Witte, C C Young, B Youngman

STANFORD U - D M Kawall

TEMPLE U - Z E Mezian

TOHOKU U - K Abe, T Akagi, M Kuriki, F Suekane, H Yuta
VIRGINIA U - T Averett, J P Chen, D G Crabb, D B Day,

E Frlež, R A Lindgren, ' $T$ J Liu, I S McCarthy, R C Minehart,

$D$ Počanić, $O A$ Rondon ( $\checkmark$ Spokesperson), L C Smith,

D Zimmerman

WISCONSIN U - II Band, J R Johnson, R Prepost, G H Zapalac

Accelerator SLAC Detector Spectrometer

Reactions Polarized beam and target

$$
\begin{array}{lc}
e^{-} \text {deut } & 9.7,16.2,29.1 \mathrm{GeV}\left(\mathrm{E}_{\mathrm{lab}}\right) \\
e^{-} p & \|
\end{array}
$$

$e^{-} p$

Particles studied $p, n$

Brief description Uses high-energy polarized electron beams and a set of ammonia based polarized proton and deuteron targets. Both parallel and perpendicular beam-target spin orientations are used. Studies the proton and neutron spin structure over the range $0.03 \leq x \leq 0.8$ at momentum transfers greater than $0.3(\mathrm{GeV} / \mathrm{c})^{2}$. Data analysis in progress (July 96 ).

Journal papers PRL 74 (1995) 346, PRL 75 (1995) 25, PL B364 (1995) 61, and PRL 76 (1996) 587.

Related experiments SLAC-E-142, SLAC-E-154, SLAC-E-155,

CERN-NA-037, CERN-NA-047

E-mail contact arnold@slac.stanford.edu, or@virginia.edu WWW Home-page

http://www.slac.stanford.edu/exp/el43/home.html

\section{SLAC-E-154}

(Proposed Oct 1993, Approved Nov 1993, Began data-taking Oct 1995, Completed data-taking Nov 1995)

PRECISION MEASUREMENT OF THE NEUTRON SPIN STRUCTURE FUNCTION USING A POLARIZED

${ }^{3} H e$ TARGET

AMERICAN U - R G Arnold, P E Bosted, J N Fellbaum,

D Reyna, S E Rock, L Sorrell, Z M Szalata, T Toole

UC, BERKELEY \& LBL - G Shapiro

CAL TECH - T Averett

CLERMON' $\Upsilon^{2}$-FERRAND U - V Breton, H Fonvieille, S Incert GRENOBLE U - M J Buenerd

KENT STATE U - B D Anderson, M Khayat, M D Manley,

$M$ Olson, G G (M) Petratos, J W Watson, W-M Zhang

MASSACHUSEITS U - C M Berisso, S Churchwell,

$Y$ Kolomensky, G Peterson

MICHIGAN U - T E Chupp, K Coulter, T B Smith, R Welsh MIT, LNS - B Brau

NIST, WASH, DC - AK Thompson

OLD DOMINION U - S E Kuhn, B Raue, F Wesselmann

PENN U - P Raines

PRINCETON U - P Bogorad, G D Cates, K Kumar,

$H$ Middleton, $M$ Romalis

RUHR U, BOCHUM - W Meyer

SACLAY - H Borel, R Lombard-Nelsen, J Marroncle, F Sabatie,

F Staley, Y Terrien

SLAC - T Akagi, P L Anthony, M Daoudi, R Erickson,

R Gearhart, R Hicks, E W Hughes ( $\checkmark$ Spokesperson)

G Igo, T Maruyama, D Miller, R Pitthan, L Rochester,

S H Rokni, J Shaw, S St Lorant, L M Stuart. T'Usher, K Witte,

C C Young, B Youngman

SMITH COLL - P Decowski

SOUIHERN OREGON STATE COLL - $T$ Marvin

SYRACUSE U $\cdots$ R Holmes, P A Souder, X.I (J) Wang

TEMPLE U .. J Martoff, Z-E Meziani, P Zyla

TOHOKU U - K Abe, M Kuriki, F Suekane, H Yuta

UCLA -- V Ghazikhanian

WILLIAM AND MARY COLL - K Griffioen

WISCONSIN U - H R Band, J R Johnson, G Mitchell, R Prepost Accelerator SLAC Detector Spectrometer

Reactions Polarized beam and target

$$
e^{-{ }^{3} \mathrm{He}} \quad 48.6 \mathrm{GeV}\left(E_{\mathrm{lab}}\right)
$$

Particles studied $n$

Brief description Measures the neutron spin structure function $g_{1}^{n}$ over $x$ ranging from 0.015 to 0.7 and $Q^{2}$ ranging from 1 


\section{SUMMARIES OF SLAC EXPERIMENTS}

to $16(\mathrm{GeV} / \mathrm{c})^{2}$. Provides aiso a precision test of the Bjorken sum rule at high average $Q^{2}$ at about $5(\mathrm{GeV} / \mathrm{c})^{2}$, and allows an extraction of the quark parton model parameters, $\Delta s$ and $\Delta q$. Uses the $82 \%$ polarized electron beam in the upgraded Aline. The target polarization is achieved by way of the optical pumping and spin exchange. Data analysis in progress (July 96). Related experiments SLAC-E-142, SLAC-E-143, SLAC-E-155,

CERN-NA-047, DESY-HERA-HERMES

E-mail contact emlyn@slac.stanford.edu

WWW Home-page

http://www.slac.stanford,edu/exp/eI54/home.html

\section{SLAC-E-155}

(Proposed Oct 1993, Approved Nov 1993, In preparation)

MEASUREMENTS OF THE NUCLEON SPIN STRUCTURE IN END STATION A AT SLAC

AMERICAN U - R G Arnold (Spokesperson), P E Bosted,

J Dunne, J Fellbaum, D Reyna, S E Rock, M Spengos,

Z M Szalata, J L White

BASEL U - A Feltham, I Sick, P Steiner, B Zihlmann

BONN U - W Meyer

CEBAF - 3 Gomez

CLERMONT-FERRAND U - V Breton, C Comptour,

H Fonvieille, Y Roblin

LIVERMORE - P L Anthony, F Dietrich

MASSACHUSETTS U, AMHERST - J Bauer, J Button-Shafer

MICHIGAN U - I' E Chupp, K P Coulter, T B Smith

NAVAL POS'TGRADUATE SCHOOL - D Garvey,

$X K$ Maruyama

OLD DOMINION U - C E Hyde-Wright, A Klein, B Raue

PENN U - R Antonov, K A Griffioen, P Raines

DAPNIA. SACL $A Y$ - T Akagi, $H$ Borel, R Erbacher, P Grenier,

R Lombard-Nelsen, J Marroncle, J Morgenstern, F M Staley,

Y D 'Terrien

SLAC - J Clendenin, G Court, M Daoudi, I Dutz, R A Gearhart,

E W Hughes, 'I Maruyama, G M G Petratos, R Pitthan,

C Prescott, A Rijllart, L S Rochester, S J St Lorant,

L M Stuart, H Tang, 'T Usher, D R Walz, K Witte, C C Young,

$B$ Youngman

STANFORD U D M Kawall, S Kuhn, Z E Meziani

TOHOKU U - K Abe, M Kuriki, F Suekane, H Yuta

VIRGINIA U - T Averett, J P Chen, D G Crabb, D B Day,

E Frlež, S Hoibraten, R A Lindgren, T J Liu, J S McCarthy

(Spokesperson), R C Minehart, J H Mitchell, D Počanić,

O A Rondon, L C Smith, D Zimmerman

WISCONSIN U - H Band, J R Johnson, R Prepost, G H Zapalac

Accelerator SLAC Detector Spectrometer

Reactions Polarized beam and target

$$
e^{-} n \quad 48.55 \mathrm{GeV}\left(\mathrm{E}_{\mathrm{lab}}\right)
$$

$e^{-} p$

$$
\text { " }
$$

Particles studied $n, p$

Brief description Measures the deep inelastic scattering of

polarized electrons from polarized ammonia targets, $\mathrm{NH}_{3}$ and $\mathrm{ND}_{3}$, to determine the spin structure functions $g_{2}$ and $g_{2}$ over $x$ ranging from 0.015 to 0.85 and $Q^{2}$ ranging from 1 to $17(\mathrm{GeV} / \mathrm{c})^{2}$. The data will double the $Q^{2}$ range of precision measurements and allow a search for nonscaling higher twist contributions to the spin structure functions. Uses a new pair of focussing magnetic spectrometers instrumented with shower counters, Cerenkov counters, and scintillator hodoscopes to measure scattered electrons and reject pions. In preparation (July 96). Scheduled to run in Jamuary 97.

Related experiments SLAC-E-142, SLAC-E-143, SLAC-E-154, CERN-NA-037, CERN-NA-047

E-mail contact arnold@slac.stanford.cdu, jsm8p@virginia.cdu

\section{SLAC-SLC-SLD}

(Proposed 1983, Approved May 1984, Began data-taking Apr 1991, In progress)

THE SLD DETECTOR FOR THE SLC
SLD COLLABORATION

BOSTON U - J A Coller, S Hedges, J T Shank, J S Whitaker

BRUNEL U - N J Allen, P Dervan, E Etzion, A Hasan,

A $K$ McKemey, C Ward, S J Watts

COLORADO U - S Fahey, U Nauenberg, D Wagner

COLORADO STATE U - M Dima, J Harton, M Smy, H Staengle,

R J Wilson

COEUMBIA U - P C Rowson

FERRARA U \& INFN, FERRARA - G Menegatti, L Piemontese

FRASCATI - A Calcaterra, R De Sangro, I Peruzzi, M Piccolo

LBL - G Shapiro, H Steiner

LOUISIANA TECH U - B Barakat, X- Jiang, K Johnston

MASSACHUSETTS U, AMHERST - G Blaylock, S S Hertzbach, R R Kofler, A Trandafir, J Wittlin

MIT - P N Burrows, R F Cowan, J Dagraca, D Dong, M J Fero,

H W Kendall, V Lia, L S Osborne, J Quigley, F E Taylor,

$E$ Torrence, $R$ Verdier, R K Yamamoto

MISSISSIPPI U - B Bolen, R Kroeger, J Reidy

MOSCOW STATE U - G Bashindzhagyan

NAGOYA U $\cdots$ K Abe, R Kajikawa, N Oishi, A Sugiyama,

S Suzuki

OREGON U ... A Arodzero, J E Brau, R Frey, J Huber,

$M$ Langston, N B Sinev, X- Yang, J- Zhou

PERUGIA U \& INFN, PERUGIA - D Falciai, G Mancinelli, G Mantovani

RUTGERS U - K G Baird, P Jacques, M Kalelkar, R J Plano, P Stamer

RUTHERFORD - C I S Damerell, D J Jackson, F J Wickens

SLAC -- 'T Akagi, D Aston, T L Barklow, J R Bogart, G R Bower, M Breidenbach ( $\checkmark$ Spokesperson), D Burke, D H Calloway,

R Casscll, A Chou, G Crawford, M Daoudi, N DeGroot,

R Dubois, M E Iuffer, J A Jaros, J Jiang, A S Johnson,

H Kawahara, M E King, D W G Leith, H L Lynch,

$T$ W Markiewicz, T Maruyama, R Messner, K C Moffeit,

D Muller, 'I I Pavcl, C Y Prescott, B N Ratcliff, L S Rochester,

I J Russell, $O$ H Saxton, J Schwiening, D Su, M Swartz,

$T$ Usher, J Va'Vra, S R Wagner, A P Waite, S H Williams,

$S$ Willocg, W J Wisniewski, M B Woods, C C Young

SOGANG U - Y- Kim, C H Park

TENNESSEE U - B Bugg, H O Cohn, E L Hart, L Kamychkov, D Onopricnko, K Shmakov, A W Weidemann

TOHOKU U - K Abe, K Hasuko, T Nagamine, S Narita,

F Suckane, $\mathfrak{J}$ Yashima, $\mathrm{f}$ Yuta

UC, SANTA BARBARA - S J Yellin

UC, SANTA CRUZ -- D G Coyme, I Fernandez, X Liu.

P L Reinertsen, T Schalk

VANDERBILT U - R S Panvini, $T$ W Reeves

WASHING'ION U, SEAT'lLE - T H Burnett, E Church, V Cook, P M Mockett, E R Weiss

WISCONSIN U - H R Band, J R Johnson, R Prepost, V Serbo, $\Upsilon$ Wright

YALE U -- C Baltay ( $\checkmark$ Spokesperson), M Liu, S Manly, T Moore, $J$ A Snyder

Accelerator SLAC-SLC Detector SLD

Reactions Polarized beam

$$
e^{+} e^{-} \quad<100 \mathrm{GeV}\left(\mathrm{E}_{\mathrm{cm}}\right)
$$

Particles studied $\quad Z^{0}$

Brief description Studies include (1) precision tests of the

Standard Model of the electroweak interactions and the $Z$ partial width to bottom statcs, particularly by measuring the left-right polarization asymmetry $A_{L R}$, (2) heavy quark physics of the $B$ system, (3) $B \bar{B}$ mixing with polarized beams, (4) tests of QCD in multi-jets, and (5) a search for new phenomena. The detector syst.cm consists of a high-precision upgraded CCD vertex detector (VXD3), a cylindrical central drift chamber with four circular endcap drift chambers, a Cerenkov ring imaging detector, fincly segmented projective tower geometry calorimetry, and a muon tracking system. Taking data (July 96). Expected to run tili March 98.

Journal papers NIM A238 (1985) 489, IEEE TNS 33 (1986) 46. IEEE TNS 33 (1986) 65, IEEE INS 33 (1986) 81, IEEE TNS 33 (1986) 113, IEEE TNS 33 (1986) 167, IEEE TNS 33 (1986) 176 , IEEE TNS 33 (1986) 194, IEEE TNS 33 (1986) 197, IEEE TNS 33 (1986) 201, IEEE TNS 33 (1986) 261, NIM A252 (1986) 295, NIM A257 (1987) 139, NIM A257 (1987) 625, IEEE TNS 35 (1988) 231, IEEE TNS 35 (1988) 282, IEEE TNS 35 (1988) 311. IEEE TNS 35 (1988) 398, NIM A264 (1988) 219, NIM A265 


\section{SUMMARIES OF SLAC EXPERIMENTS}

(1988) 99, NIM A273 (1988) 858, IEEE TNS 36 (1989) 23, IEEE TNS 36 (1989) 276, IEEE TNS 36 (1989) 339, IEEE TNS 36 (1989) 595, IEEE TNS 36 (1989) 675, IEEE TNS 36 (1989) 751, IEEE TNS 36 (1989) 822, IEEE TNS 36 (1989) 1657, NIM A275 (1989) 484, NIM A276 (1989) 94, NIM A277 (1989) 222, NIM A283 (1989) 582, NIM A283 (1989) 590, NIM A284 (1989) 339, IEEE TNS 37 (1990) 1132, IEEE TNS 37 (1990) 1191, NIM A288 (1990) 236, NIM A289 (3990) 449, NIM A289 (1990) 463 NIM A289 (1990) 577, NIM A290 (1990) 353, NIM A293 (1990) 136, IEEE TNS 38 (1991) 348, NP (PROC SUPPL) 23B (1991) 219, NP (PROC SUPPL) 23B (1991) 227, NIM A300 (1991) 501, NIM A328 (1993) 472, MPL A8 (1993) 2237, PRL 70 (1993) 2515, PRL 71 (1993) 2528, NP (PROC SUPPL) 37B (1994) 23, PRL 72 (1994) 3145, PRL 73 (1994) 25, PR D50 (1994) 5580 , NP (PROC SUPPL) 39BC (1995) 121, PRL 74 (1995) 1512, PRL 74 (1995) 2880, PRL 74 (1995) 2890, PRL 74 (1995) 2895, PRL 75 (1995) 3609, PRL 75 (1995) 3624, PRL 75 (1995) 4173, PR D51 (1995) 962, PR D52 (1995) 4240, PR D52 (1995) 4828, PL B371 (1996) 149, PR D53 (1996) 1023, and PR D53 (1996) 2271.

Related experiments CERN-LEP-ALEPH, CERN-LEP-DELPHI, CERN-LEP-L3, CERN-LEP-OPAL

E-mail contact mib@slac.stanford.edu, baltay@yalph 2 .physics.yale.edu

WWW Home-page

http://www-sld.slac.stanford.edu/sldwww/sld.html

\section{SLAC-PEP2-BABAR}

(Proposed Jun 1994, Jun 1994, Approved Jan 1996, In preparation)

\section{THE B FACTORY DETECTOR}

BABAR COLLABORATION

Accelerator SLAC-PEP2 Detector BABAR

Brief description BABAR is a detector designed to exploit the single intcraction region in the PEP2 facility. The primary aim is a detailed study of $C P$ violating asymmetries in $B^{0}$ meson decay, but a wide variety of other $B$, charm, $\tau$, and twophoton physics will also be accessible. The detector comprises a nested set of detector subsystems to (1) precisely measure the collision point and decay points of the $B$ meson pairs produced, (2) measure the momentum and energy of the decay particles with a high resolution, and (3) precisely identify particles in these decays (i.e. electrons, muons, pions, kaons). The machine will be commissioned Fall 1998 and the detector should be ready to begin the studies in early 1999. Under construction (July 96). The collaboration consists of more than 500 scientists, engineers, and graduate students from 10 countries. For further details, please contact the Spokesperson, Prof. David G. Hitlin [Cal Tech]. The Deputy Spokesperson is Dr. Roy Aleksan [Saclay].

E-mail contact hitlin@slac.stanford.edu WWW Home-page

http://www.slac.stanford.edu/BF/doc/www/bfHome.html

\section{Other SLAC Experiments}

Listed here are some other SLAC experiments of interest to the particle physics community. Find more details about these projects online, in the SLAC's EXPERIMENTS database (see p. 3), or contact the spokespersons.

SLAC-E-140X (Completed data-taking Sep 1991)

MEASUREMENT OF THE $x, Q^{2}$, AND HYDROGEN DEUTERIUM DEPENDENCE OF $R=\sigma_{L} / \sigma_{T}$

Spokespersons: Stephen E. Rock [American U.], Arie Bodek [Rochester U.]

E-mail contact ser@slac.stanford.edu,

bodek@urhep.pas.rochester.edu
SLAC-E-144 (Taking data)

STUDY OF QED AT CRITICAL FIELD STRENGTH IN INTENSE LASER - HIGH-ENERGY ELECTRON COLLISIONS AT SLAC

By E144 Collaboration

Spokespersons: Kirk T. McDonald [Princeton U.]. Adrian C. Melissinos [Rochester U.], David L. Burke [SLAC]

E-mail contact mcdonald@puphep.princeton.edu, meliss@urhcp.pas.rochester.edu, daveb@slac.stanford.edu WWW Home-page

http://www.slac.stanford.edu/exp/e144/e144.html

SLAC-E-146 (Completed data-taking Apr 1993)

STUDY OF THE INTERFERENCE BETWEEN MULTYPLE SCATTERING AND BREMSSTRAHLUNG (LPM EFFECT)

Spokesperson: Spencer R. Klein [LBL, Berkeley]

$\underline{\text { E-mail contact }}$ srklein@lbl.gov

SLAC-NE-17 (Completed data-taking Oct 1991)

TWO-BODY PHOTODISINTEGRATION OF THE

DEUTERON AT FORWARD ANGLES BETWEEN 1.0 AND $3.0 \mathrm{GeV}$

Spokesperson: Roy J. Holt [Argonnc]

E-mail contact r-holt@uiuc.edu

SLAC-NE-18 (Completed data-taking Oct 1991)

MEASUREMENT OF THE NUCLEAR DEPENDENCE AND MOMENTUM TRANSFER DEPENDENCE OF QUASIELASTIC $\left(e, e^{\prime} p\right)$ SCATTERING AT LARGE MOMENTUM TRANSFER

Spokespersons: Richard G. Milner [MIT, LNS], Bradley W. Filippone [Cal Tech]

E-mail contact milner@mitlns.mit.edu, brad@erin.caltech.edu

\section{SLAC Future Plans}

In the next few years SLAC will continue to support a series of complementary high-energy physics programs: (1) studies of the spin structure of nucleons using the high current, high energy, polarized electron-beam scattering from polarized neutron and proton targets, (2) study of the electro-weak interaction with the SLD detector through precision measurements at the $Z$ pole, using polarized electron collisions on unpolarized positrons at the energy of the $Z,(3)$ accelerator $R \& D$ towards a real linear collider (NLC). (4) preparations to study time-dependent $X$-ray sources with the USA experiment to be launched in 1997, and R\&D towards a new space experiment studying high-energy $\gamma$ rays - a follow on to the very successful EGRET experiment now orbiting earth, and (5) activity related to the development of the far-future high-gradient accelerators. The spin structure and SLD experiments are scheduled to run through March 1998. 'I'he asymmetric $B$ Factory (PEP2) is expected to be commissioned before the end of that year, and the BaBar detector should begin its study of the $C P$-violation phenomena in early 1999. 


\section{SUMMARIES OF TRIUMF EXPERIMENTS}

\section{Selected TRIUMF Experiments}

\section{TRIUMF-497-287}

(Proposed Oct 1987, Approved Dec 1987, Began data-taking 1995, In progress)

MEASUREMENT OF THE FLAVOR-CONSERVING HADRONIC WEAK INTERACTION

\section{PARITY COLLABORATION}

MANITOBA U - J Birchall ( $\checkmark$ Spokesperson), J R Campbell, A A Green, A Hamian, L R Lee, S A Page ( $\checkmark$ Spokesperson), W D Ramsay, S D Reitzner, V Sum, W T H van Oers $(\checkmark$ Spokesperson), R Woo

LOS ALAMOS - J D Bowman, $\mathrm{R}$ E Mischke

TRIUMF - C A Davis, D C Healey, R Helmer, P Levy,

P W Schmor

ALBER'TA U ‥P W Green, E Korkmaz, G Roy, J Soukup,

G M Stinson

CARNEGIE MELLON U - A Berdo'

MOSCOW, INR - Y Kuznetzov, N Titov, S Zadorozny, A Zelenski

Accelerator TRIUMF Detector Ionization

Reactions Polarized beam

$$
p p \rightarrow p p \quad 221 \mathrm{MeV}\left(\mathrm{T}_{\mathrm{lab}}\right)
$$

Brief description Measures the parity violating (PV) longitudinal analyzing power $A_{z}$ and the weak meson-nucleon coupling constant $h_{\rho}{ }^{p p}$. Uscs a new beamline dedicated to the PV measurements, and a liquid hydrogen target. In progress (July 96)

Journal papers PR D37 (1988) 1769, NIM A307 (1991) 26, and NP $A 553$ (1993) 823c.

E-mail contact birchall@physics.umanitoba.ca, shelley@triumf.ca, vanoers@triumf.ca

\section{TRIUMF-537}

(Proposed May 1988, Completed data-taking Jun 1991)

\section{RADIATIVE DECAX OF THE $\triangle$ RESONANCE}

BRITISH COLUMBIA U - D F Measday (Spokesperson),

$S$ Stanislaus, $P$ Weber

KENTUCKY U - M A Kovash

NEW MEXICO $U$.. B Bassalleck

BOSTON U - E C Booth, J P Miller

Accelerator TRIUMF Detector Photon spectrometer

Reactions Polarized target

$$
\pi^{-} p \rightarrow n \gamma \quad 100-250 \mathrm{MeV}\left(\mathrm{T}_{\mathrm{lab}}\right)
$$

$\pi^{-} p \rightarrow \pi^{0} n$

$$
"
$$

Brief description Measures $\Delta^{0}$ radiative decay multipoles and differential cross-sections. A polarized target has been successfully used in phase-II of the experiment. Data analysis in progress (July 96 ).

E-mail contact measday@triumf.ca

\section{TRIUMF-614}

(Proposed Jun 1990, Approved Dec 1993, In preparation)

\section{PRECISION MEASUREMENT OF THE MICHEL} PARAMETERS IN $\mu^{+}$DECAY

TRIUMF - R Abegg, M Comyn, D R Gill ( $\sqrt{ }$ Spokesperson), $P$ Gumplinger, R Helmer, J Macdonald, J-M Poutissou, R Poutissou, G Wait, D Wright

TEXAS A AND M - C Gagliardi ( $\checkmark$ Spokesperson), R Tribble ( $\sqrt{ }$ Spokesperson) BRITISH COLUMBIA U - M Hasinoff

VALPARAISO U, INDIANA - D Koetke, $R$ Manweiler KURCHATOV INST, MOSCOW - A Khruchinsky, Y Lechin, L Miassoedov, V Selivanov ( $\checkmark$ Spokesperson), I Sinitzin,

Torokhov
ALBERTA $U$ - N Rodning SASKATCHEWAN U - Y M Shin REGINA U \& TRIUMF - $\mathrm{R}$ Tacik

Accelerator TRIUMF Detector Spectrometer

Brief description Tests the right-left models of weak interactions. A surface muon beam is used as a source of muons with polarization almost completely (better than $99.99 \%$ ) opposite to the muon momentum. The experimental apparatus is contained in a superconducting solenoid with a maximum field of $2.3 \mathrm{~T}$, collinear to the muon polarization. Muons stop in a thin planar aluminum target located in the center of the magnet, and the resulting positrons are recorded by one of the two planar drift chamber (PDC) assemblies located on both sides of the target. Under construction. Data taking expected in Fall 1997

Related experiments LAMPF- 1240

E-mail contact drgill@triumf.ca, tribble@comp.tamu.edu, svi@slv kiaesu

\section{TRIUMF-645}

(Proposed Jun 1991, Approved Jun 1991, Began data-taking May 1992, Completed data-taking Jun 1992)

\section{ABSOLUTE DIFFERENTIAL CROSS-SECTIONS} IN THE $\pi^{ \pm} p \rightarrow \pi^{ \pm} p$ REACTION AROUND THE $\triangle$ RESONANCE

PISCAT COLLABORA'TION

BRITISH COLUMBIA U - F Duncan, A Feltham, G Jones,

$J$ Lange, M M Pavan ( $\checkmark$ Spokesperson), K Raywood, M E Sevior TRIUMF - R Adams, J $\Upsilon$ Brack ( $\checkmark$ Spokesperson), D Ottewell,

G R Smith, B Wells

REGINA U .. E L Mathie, R Tacik

COLORADO U - R A Ristinen

KARLSRUHE U - H M Staudenmaier

ST PETERSBURG, INP - I I Strakovsky

SIMON FRASER U - R Helmer

Accelerator TRIUMF Detector Scintillator

\section{Reactions}

$$
\pi^{+} p \rightarrow \pi^{+} p \quad 141-267 \mathrm{MeV}\left(\mathrm{T}_{\mathrm{lab}}\right)
$$$$
\pi^{-} p \rightarrow \pi^{-} p
$$

Brief description Uses flat, solid $\mathrm{CH}_{2}$ (polyethylene) targets as well as a supercooled flat-window liquid hydrogen target. Scintillator telescopes are used for coincidence detection of pions and protons. Covers angular range between $30^{\circ}$ and $160^{\circ}$ c.m. Anticipates $1-1.5 \%$ statistical and $1.5-2.0 \%$ systematic uncertainties. Data analysis completed and a publication being prepared (July 96 ).

Related experiments TRIUMF-322, TRIUMF-471

E-mazl contact pavan@mitlns.mit.edu,mpavan@bun.mit.edu. brack@spectr.colorado.edu

\section{TRIUMF-703}

(Proposed May 1993, Approved Jul 1993, Began data-taking Dec 1993, In progress)

\section{PION LIFETIME MEASUREMENT}

TRIUMF - D A Bryman, M Fujiwara, I A Macdonald, G Marshal, T Numao ( $\checkmark$ Spokesperson), A Olin

Accelerator TRIUMF Detector Counter

Reactions

$$
\pi^{+} \rightarrow e^{+} \nu
$$

Particles.studied $\pi^{+}$

Bref description The pion lifetime is measured by observing the time dependence of the surface muon yield. Taking data (July 96).

Journal papers PR D52 (1995) 4855.

Related experiments TRIUMF-248

E-mail contact toshio@triumf.ca 


\section{Other TRIUMF Experiments}

Listed here are some other TRIUMF experiments of interest to the particle physics community. Find more details about these projects online, in the SLAC's EXPERIMENTS database (see p. 3), or contact the spokespersons. Check also the yearly TRIUMF publication 'Annual Report of Scientific Activities', which lists all the Lab's ongoing experiments.

TRIUMF-369 (Completed data-taking Mar 1993)

CHARGE SYMMETRY BREAKING IN $n p$ ELASTIC SCATTERING AT $350 \mathrm{MeV}$

Spokespersons: Willem T.K. van Oers [Manitoba U.],

L.Gordon Greeniaus [Alberta U.]

E-mail contact gree@phys.ualberta.ca, vanoers@triumf.ca

TRIUMF-372 (Completed data-taking Feb 1991)

SINGLE PION PRODUCTION IN $n p$ SCATTERING

Spokesperson: Norman E. Davison [Manitoba U.]

E-mail contact davison@umphys.physics.umanitoba.ca

TRIUMF-445 (Completed data-taking Dec 1993)

POLARIZATION MEASUREMENT IN THE ${ }^{3} \mathrm{He}\left(\pi^{+}, \vec{p} p\right) p$ REACTION

Spokespersons: D. Ashery, S. MayTal-Beck [Tel Aviv U.]

$\underline{\text { E-mail contact }}$ ashery@tauphy.tau.ac.il, ashery@triumf.ca

TRIUMF-452 (Completed data-taking Feb 1994)

RADXATIVE MUON CAPTURE ON HYDROGEN

By RMC Collaboration

Spokespersons: Michael D. Hasinoff [British Columbia U.], Georges Azuelos [Montreal U.]

E-mail contact miha@triumf.ca, azuelos@lps.umontreal.ca

TRIUMF-482 (Completed data-taking Sep 1991)

MEASUREMENTS OF SPIN TRANSFER COEFFI-

CIENTS IN $p d$ ELASTIC SCATTERING

Spokesperson: Rudolf Abegg [TRIUMF]

E-mail contact abegg@triumf.ca

TRIUMF-498 (Completed data-taking 1993)

ANALYZING POWER ZERO CROSSING ANGLES IN $n p$ ELASTIC SCATTERING BELOW $300 \mathrm{MeV}$

Spokesperson: Charles A. Davis [TRIUMF]

E-mail contact cymru@triumf.ca

TRIUMF-502 (Completed data-taking 1992)

MEASUREMENT OF ANALYZING POWERS IN LOW ENERGY $\pi d$ ELASTIC SCATTERING

Spokesperson: N.R. Stevenson [Saskatchewan U.]

TRIUMF-506 (Completed data-taking 1991)

LOW ENERGY $\pi d \rightarrow p p$ ANALYZING POWERS

Spokesperson: Edward L. Mathie [Regina U.]

E-mail contact mathie@meena.cc.uregina.ca, mathie@triumf.ca

\section{TRIUMF-508}

STUDY OF THE $\pi^{+} d \rightarrow \pi^{-} \pi^{+} p p$ REACTION AT $T=240$ $\mathrm{MeV}$

Spokesperson: Rinaldo Rui [Trieste U.]

E-mail contact rui@triumf.ca

\section{TRIUMF-557 (Completed data-taking 1992)}

ELASTIC SCATTERING OF $100 \mathrm{MeV} \pi^{+}$FROM A POLARIZED ${ }^{3}$ He TARGET

Spokespersons: Otto F. Haeusser ['TRIUMF and Simon Fraser U.], B. Larson [Simon Fraser U.]

E-mail contact hausser@triumf.ca

TRIUMF-560 (Completed data-taking 1994)

LOW ENERGY $\pi^{+} p$ ANALYZING POWERS WITH CHAOS

Spokesperson: G.R. Smith [TRIUMF]

E-mail contact smith@erich.triumf.ca

TRIUMF-561 (Completed data-taking Feb 1991)

THRESHOLD MEASUREMENTS OF $\mathrm{H}\left(\pi^{-}, \pi^{+} \pi^{-}\right) n$ AND $\mathrm{H}\left(\pi^{+}, \pi^{+} \pi^{+}\right) n$

Spokesperson: Martin E. Sevior [British Columbia U.]

E-mail contact msevior@triumf.ca

TRIUMF-566

ELASTIC PROTON SCATTERING FROM POLARIZED ${ }^{3} \mathrm{He}$

Spokesperson: Otto F. Haeusser [Simon Fraser U. and TRIUMF]

E-mail contact hausser@triumf.ca

TRIUMF-570 (Completed data-taking Jan 1993)

GAMMA-NEUTRINO ANGULAR CORRELATION IN MUON CAPTURE ON ${ }^{28} \mathrm{Si}$

Spokesperson: David S. Armstrong [LBL, Berkeley]

E-mail contact armd@newton.physics.wm.edu, armd@reg,triumf.ca

TRIUMF-592 (Completed data-taking 1995)

RADIATIVE MUON CAPTURE ON ${ }^{3} \mathrm{He}$

By RMC Collaboration

Spokesperson: Dennis H. Wright [TRIUMF]

E-mail contact wright@triumf.ca

TRIUMF-598 (Completed data-taking Aug 1992)

INTEGRAL CROSS-SECTIONS FOR THE $\pi^{+} p$ INTERACTION IN THE 3, 3 RESONANCE REGION

Spokesperson: Eli Friedman [Hebrew U.]

$\underline{\text { E-mail contact }}$ elifried@vms.huji.ac.il

TRIUMF-612 (Completed data-taking 1994)

HYPERFINE DEPENDENCE OF EXCLUSIVE MUON

CAPTURE ON ${ }^{19} \mathrm{~F},{ }^{23} \mathrm{Na},{ }^{27} \mathrm{Al},{ }^{35} \mathrm{Cl}$. AND ${ }^{37} \mathrm{Cl}$

Spokesperson: Tim P. Gorringe [Kentucky U.]

E-mail contact gorringe@ukcc.uky.edu, gorringe@triumf.ca 
SUMMARIES OF TRIUMF EXPERIMENTS

TRIUMF-624 (Completed data-taking Aug 1994)

THE $(\pi, 2 \pi)$ REACTION, A TOOL TO DETERMINE SCATTERING LENGTHS AND COUPLING CONSTANTS

Spokespersons: Richard R. Johnson, Martin E. Sevior [British Columbia U.], Nevio Grion, Rinaldo Rui [Trieste U.]

E-mail contact johnson@physics.ubc.ca, rrjohnson@triumf.ca, msevior@triumf.ca, grion@trieste.infn.it, grion@triumf.ca, rui@triumf.ca

TRIUMF-630 (Completed data-taking 1994)

ELASTIC PROTON SCATTERING FROM SIDEWAYS AND LONGITUDINALLY POLARIZED ${ }^{3} \mathrm{He}$

Spokesperson: D.M. Whittal [Simon Fraser U.]

TRIUMF-633

MEASUREMENT OF $p p \rightarrow p n \pi^{+}$AT 420 AND $500 \mathrm{MeV}$ Spokesperson: Kenneth H. Hicks [Ohio U.]

E-mail contact hicks@ouvaxa.cats.ohiou.edu, hicks@triumf.ca

TRIUMF-643 (Completed data-taking 1992)

TEST OF THE LOW ENERGY THEOREM FOR RADIATIVE PION CAPTURE

Spokespersons: David A. Hutcheon [TRIUMF], Michael A. Kovash [Kentucky U.]

E-mail contact smurf@triumf.ca, kovash@ie.pa.uky.edu, kovash@triumf.ca, phy133@ukcc.uky.edu

TRIUMF-653 (Completed data-taking Aug 1993)

MEASUREMENT OF THE $\pi^{+} \pi^{-}$INVARIANT MASS IN NUCLEI AS A TOOL FOR DETERMINING THE MASS DISTRIBUTION OF THE $\sigma$ MESON

Spokespersons: Nevio Grion, Rinaldo Rui [Trieste U.]

E-mail contact grion@triumf.ca, grion@trieste.infn.it, rui@triumf.ca

TRIUMF-661 (Completed data-taking Jun 1994)

NEUTRON-NEUTRON SCATTERING LENGTH VIA

$\pi^{-} d \rightarrow$ ynn

Spokesuerson: Michael A. Kovash [Kentucky U.]

E-mail contact kovash@triumf.ca, kovash@ic.pa.uky.edu, phy133@ukcc.uky.edu

TRYUMF-683 (Completed data-taking Sep 1994)

ASYMMETRIES FOLLOWING MUON CAPTURE BY POLARIZED MUONIC ${ }^{3} \mathrm{He}$

Spokespersons: W. Cummings [Simon Fraser U.], O.F. Hausser

[Simon Fraser U. and TRIUMF]

E-mail contact hausser@triumf.ca

TRIUMF-704 (In preparation)

CHARGE SYMMETRY BREAKING IN $n p \rightarrow d \pi^{0}$ CLOSE TO THRESHOLD

By SASP-CSB Collaboration

Spokespersons: Allena K. Opper [Alberta U.], Elie Korkmaz [Northern British Columbia U.]

E-mazlcontact opper@phys.ualberta.ca, korkmaz@unbc.edu
TRIUMF-719 (Completed data-taking 1995)

${ }^{4} \mathrm{He}\left(\pi^{+}, \pi^{-} p p\right)$ INVARIANT MASS MEASUREMENT WITH CHAOS

Spokesperson: Greg R. Smith [TRIUMF]

E-mail contact smith@triumf.ca 


\section{Nonaccelerator Experiments}

\section{UNDERGROUND-BOREXINO}

(In preparation)

BOREXINO: A SOLAR NEUTRINO EXPERIMENT AT GRAN SASSO

BOREXINO COLLABORATION

Brief description BOREXINO is one of the 'next generation' solar neutrino projects at the Gran Sasso Laboratory. Uses a Pseudocumene based liquid scintillator as active medium. Measures the ${ }^{7}$ Be line neutrino flux (energy $=0.861 \mathrm{MeV}$ ). It is very sensitive to the neutrino oscillations in both the scenarios of vacuum oscillations and the MSW effect. Tests of the feasibility of the project have been successfully completed. For further information, please contact the Spokesperson, Prof. Gianpaolo Bellini [Milan U. and INFN, Milan].

Related experiments SAGE, GALLEX, SUDBURX, KAMIOKANDE, SUPERKAMIOKANDE

E-mail contact bellini@mi.infn.it, bellini@lngs.infn.it WWW Home-page

http://www.lngs.infn.it/lngs/htexts/borex/html/borex.html

\section{UNDERGROUND-GALLEX}

(Proposed 1983, Approved Apr 1985, Began data-taking May 1991, In progress)

\section{GALLIUM EUROPEAN EXPERIMENT}

GALLEX COLLABORATION

HEIDELBERG, MAX PLANCK INST - W Hampel, G Heusser,

3 Kiko, T Kirsten ( $\checkmark$ Spokesperson), M Laubenstein,

E Pernicka, W Rau, U Roenn, M Wojcik, Y Zakharov

FORSCHUNGSZENTRUM, KARLSRUHE - K Ebert, T Fritsch,

D Heidt, E Henrich, L Stieglitz, R von Ammon, F Weirich GRAN SASSO - M Balata, F X Hartmann, M Sann

MILAN U - E Bellotti, C Cattadori, O Cremonesi, N Ferrari,

E Fiorini, L Zanotti

MUNICH, TECH U - M Altmann, R Moessbauer, F von Feilitzsch

NICE OBSERVATORY - G Berthomicu, E Schatzman

ROME U \& INFN, ROME - C Bacci, P Belli, R Bernabei,

$S$ D'Angelo, L Paoluzi

SACLAY - A Bevilacqua, M Cribier, L Gosset, J Rich, M Spiro, C Tao, D Vignaud

BROOKHAVEN - 3 Boger, R L Hahn, J K Rowley, R W Stoenner, J Weneser

Accelerator NONE Detector Counter

Reactions

$\nu_{e}{ }^{71} \mathrm{Ga} \rightarrow e^{-71} \mathrm{Ge}$

Particles studied $\nu_{e}$

Brief description This is a radiochemical neutrino experiment.

Uses 30 tons of gallium in the $8.2-$ molar $\mathrm{GaCl}_{3}$ solution.

Installed in the South Wing of Hall $\mathrm{A}$ of the Gran Sasso

Laboratory. Ias an overhead shielding of about $3400 \mathrm{~m}$ of water equivalent. An interaction with neutrinos effectively transforms gallium chloride into $\mathrm{GeCl}_{4}$, which is then extracted from the solution with an appropriate gas purging system. Counted in extremely low-level proportional counters. Sensitive to the lowenergy neutrinos produced by $p p$ fusion in the Sun. Designed for an order of one event per day. Taking data (July 96).

Journal papers NIM A274 (1989) 203, PL B285 (1992) 376, PL

B285 (1992) 390, NIM A329 (1993) 541, PL B314 (1993) 445, PL B327 (1994) 377, NP (PROC SUPPL) 35 (1994) 418, PL

B342 (1995) 440, PL B357 (1995) 237, and ASPP 4 (1995) 23.

Related experiments SAGE, BOREXINO

E-mail contact kirst@kosmo.mpi-hd.mpg.de WWW Home-page

http://kosmopc.mpi-hd.mpg.de/gallex/gallex.htm

\section{UNDERGROUND-HOMESTAKE-} CHLORINE

(Proposed 1965, Approved 1965, Began data-taking 1970, In progress)

THE HOMESTAKE CHLORINE SOLAR NEUTRINO EXPERIMENT

PENN U - B T Cleveland, T Daily, R Davis, Jr

( $\sqrt{ }$ Spokesperson), K Lande ( $\checkmark$ Spokesperson), C K Lee,

$\mathrm{P}$ Wildenhain

LEHMANN COLL - J Ullman

Accelerator NONE Detector Counter

Reactions

$$
\nu_{e}{ }^{37} \mathrm{Cl} \rightarrow e^{-37} \mathrm{Ar}
$$

Particles studied $\nu_{e}$

Brief description The ${ }^{37} \mathrm{Cl}$ solar neutrino detector in the Homestake Gold Mine consists of 615 tons of tetrachloroethylene $\left(\mathrm{C}_{2} \mathrm{Cl}_{4}\right), 4200 \mathrm{~m}$ of water equivalent underground. It uses radiochemical techniques to determine the ${ }^{37} \mathrm{Ar}$ production rate. The detector was built at BNL in 1965-67 and operated by Brookhaven until 1984. At that time the laboratory was transferred to Penn U. Collecting data regularly since 1970.

Journal papers PRL 47 (1981) 1507, and ASTJ (1996) (to be published).

E-mail contact klande@mail.sas.upenn.edu

\section{UNDERGROUND-HOMESTAKE-IODINE}

(Proposed 1993, Approved 1994, In preparation)

THE HOMESTAKE IODINE SOLAR NEUTRINO EXPERIMENT

PENN U - B T Cleveland, T Daily, $R$ Davis, $I r, \underline{K}$ Lande ( $\sqrt{ }$ Spokesperson), C K Lee, $P$ Wildenhain ( $\sqrt{\text { Spokesperson) }}$ LEHMANN COLL ... I Ullman

LOS ALAMOS - R Burman

WASHINGTON U, SEATTLE - W Haxton

MOSCOW, INR - V N Gavrin

Accelerator NONE Detector Counter

Reactions

$\nu_{e}^{127} \mathrm{I} \rightarrow e^{-127} \mathrm{Xe}$

Particles studied $\nu_{e}$

Brief description The ${ }^{127} \mathrm{I}$ solar neutrino experiment in the

Homestake Gold Mine uses a detector medium of sodium iodide dissolved in water. The total detector mass of the unit now under construction will be 235 tons of which 100 tons is ${ }^{127} \mathrm{I}$. The detector is located $4200 \mathrm{~m}$ of water equivalent underground. It uses radiochemical techniques to determine the ${ }^{127} \mathrm{Xe}$ production rate. The detector, which has an extraction time constant of 11 minutes, will be fully automated and will operate under complete computer control. Two extractions will be carried out per day to search for a Day/Night effect for electron neutrinos from the decay of ${ }^{7} \mathrm{Be}$. Expected to begin data taking at the end of 1996.

Related experiments LAMPF-1213, IUCF-E-373

E-mail contact klande@mail.sas.upenn.edu, paul@goodricke.astro.upenn.edu

\section{UNDERGROUND-ICARUS}

(Approved 1995, In preparation)

ICARUS: IMAGING COSMIC AND RARE UNDERGROUND SIGNAL

CERN - P Cennini, S Cittolin, G Maurin, A Placci, J P Revol, C Rubbia ( $V$ Spokesperson), W H Tian

AQUILA U \& INFN, AQUILA - F Cavanna. E Olejarczyk, G Piano Mortari, M Verdecchia 


\section{SUMMARIES OF NONACCELERATOR EXPERIMENTS}

BEIJING, IHEP - L K Ding, Y Li, F Lu, J M Ma, J F Qiu, HY Y Sheng, $K$ L Tung, J Y Zeng, B Zhang

FRASCATI - F Casagrande, D Dzialo-Giudice, $X \mathrm{Li}$,

G Mannocchi, S Motto, P Picchi

UCLA - D Cline, W Hong, G Muratori, S Otwinowski, J Park, II G Wang, M Zhou

PADUA U \& INFN, PADUA - A Bettini, C Carpanese, S Centro, C De Vecchi, D Pascoli, A Pepato, F Pietropaolo, $S$ Ventura

PAVYA U \& INFN, PAVIA - P Benetti, E Calligarich, R Dolfini, A Gigli-Berzolari, F Mauri, L Mazzone, C Montanari,

A Piazzoli, A Rappoldi, G L Raselli, M Rossella, D Scannicchio, C Vignoli

PISA U \& INFN, PISA - F Sergiampietri

TURIN, COSMO-GEOFISICA LAB - L Periale, S Suzuki

TEXAS U, DALLAS - E Feynyves

MILAN U \& INFN, MLLAN - D Cavalli, S Cesana, A Ferrari,

F Gianotti, S Resconi, P Sala, M Terrani

Accelerator NONE Detector ICARUS

Particles studied $p, \nu$

Brief description The first phase of the project includes the construction of a 600-ton detector at Gran Sasso Laboratory. This could be done within the next three years. In the second phase, a 5,000-ton detector will be built. The ICARUS detector represents a new generation of bubble chambers, and will use the ultra-pure liquid argon and the readout technique (LArTPC) of ionization data. It is designed to provide threedimensional views of ionizing events with particle identification from $\mathrm{d} E / \mathrm{d} x$ and range measurements. It is also a homogeneous calorimeter of very fine granularity and high accuracy, and thus a good tool for rare event searches, such as proton decays. Other topics covered include atmospheric neutrino studies and solar neutrino detection (above $5 \mathrm{MeV}$ ). It is expected that the detector will be used in a long baseline neutrino oscillation experiment with the $\nu$ beam from CERN (732-km to the North). Under construction (July 96).

Journal papers NIM A315 (1992) 223, NIM A327 (1993) 173,

NIM A332 (1993) 395, NIM A333 (1993) 567, NIM A345 (1994)

230, NIM A346 (1994) 550, NIM A355 (1995) 660, NIM A356

(1995) 507, and NIM A356 (1995) 526.

Related experiments SUPERKAMIOKANDE

E-mail contact carlo.rubbia@cern.ch

WWW Home-page http://www.aquila.infn.it/icarus/

\section{UNDERGROUND-KAMIOKANDE-III}

(Began data-taking Nov 1985, Completed data-taking Apr 1996)

\section{THE KAMIOKANDE EXPERIMENT}

TOKYO U, ICRR - Y Fukuda, T Hayakawa, K Inone, T Ishida,

$\mathrm{K}$ Ishihara, H Ishino, S Joukou, T Kajita, S Kasuga, Y Koshio, T' Kumita, K Matsumoto, M Nakahata, K Nakamura,

K Okumura, A Sakai, M Shiozawa, I Suzuki, Y Suzuki,

$T$ Tomoeda, Y Totsuka ( $\sqrt{ }$ Spokesperson)

TOKYO U, INS - E Ichihara, S Miyamoto, K Nishikawa

KEK -.. K S Hirata, K Kihara, Y Oyama

TOKAI U, SHIBUYA - T Horiuchi, M Koshiba, K Nishijima KOBE U -.. T Suda, A T Suzuki

NIIGATA U - T Ishizuka, K Miyano, H Okazawa

OSAKA U - T Hara, Y Nagashima, M Takita, $T$ Yamaguchi

TOKYO INST TECH - Y Hayato, K Kaneyuki, T Suzuki,

Y Takeuchi, 'T Tanimori

GIFU U $\cdots$ S Tasaka

TOHOKU U - K Fujita, S Hatakeyama, M Koga, S Maruyama,

A Suzuki

MIYAGI U OF EDUCATION - S Mori

Accelerator NONE Detector Counter

Reactions

$$
\nu e^{-} \rightarrow \nu c^{-}
$$$$
\bar{\nu}_{c} p \rightarrow n e^{+}
$$

Particles studzed $\quad p, n$, monopole, muon, $\nu$

Brief description A 3000-ton water C̆erenkov detector, $2700 \mathrm{~m}$ of water equivalent underground. The KAMIOKANDE-I detector has been upgraded with new electronics, TDC's, and one thousand 20 -inch phototubes surrounded by aluminized reflectors. Studies nucleon decays, solar, supernova, atmospheric and high-energy cosmic neutrinos, high-energy muons, etc. The second phase was completed in April 90, the third phase. started in October 90. Data taking officially ended in April 96, but the detector is still capable of recording possible supernova neutrinos. Data analysis in progress (July 96).

Journal papers NIM 205 (1983) 443, JPSJ 54 (1985) 3213, JPSJ 54 (1985) 4065, PRL 56 (1986) 991, JPSJ 55 (1986) 711, JPSJ 55 (1986) 3786, PR D34 (1986) 902, PRL 58 (1987) 1490, PRL 59 (1987) 2604, PR D36 (1987) 3537, PL B205 (1988) 416, PRL 61 (1988) 385, PRL 61 (1988) 2653, PR D38 (1988) 448, PL B220 (1989) 308, PRL 63 (1989) 16, PR D39 (1989) 1481, ASTJ 359 (1990) 574, PRL 65 (1990) 1297, PRL 65 (1990) 1301, PL B270 (1991) 89, PRL 66 (1991) 9, JPSJ 60 (1991) 2808, PR D43 (1991) 2843, PR D44 (1991) 617, PR D44 (1991) 2220, PR D44 (1991) 2241 [erratum: PR D45 (1992) 2170], NLM A320 (1992) 310, PL B278 (1992) 217, PL B280 (1992) 146, PL B283 (1992) 446, PL B289 (1992) 463, PR D45 (1992) 3355, NIM A329 (1993) 299, PL B311 (1993) 357, NP (PROC SUPPL) 31 (1993) 105, PR D48 (1993) 5505, NIM A340 (1994) 612, PL B335 (1994) 237, ASTJ 435 (1994) 225, and PL B374 (1996) 238 .

Related experiments KEK-261A

E-mail contact totsuka@sukip04.icrr.u-tokyo.ac.jp WWW Home-page

http://www-sk.icrr.u-tokyo.ac.jp/doc/kam/index.html

\section{UNDERGROUND-SUPERKAMIOKANDE}

(Proposed 1986, Approved 1991, Began data-taking Apr 1996, In progress)

\section{THE SUPER-KAMIOKANDE SOLAR NEUTRINO AND} NUCLEON DECAY DETECTOR

TOKYO U, ICRR - Y Fukuda, T Hayakawa, K Inoue, K Ishihara, K Ishino, Y Itow, T Kajita, J Kameda, S Kasuga, Y Koshio, K Martens, M Miura, M Nakahata, M Oketa, K Okumura, M Ota, N Sakurai, M Shiozawa, Y Suzuki, Y Takeuchi, Y Totsuka ( $\checkmark$ Spokesperson)

KEK - J Kanzaki, K Nakamura, Y Oyama, M Sakuda, O Sasaki

TOHOKU U -. K Fujita, A Hasegawa, T Hasegawa,

$S$ Hatakeyama, T Iwamoto, T Kinebuchi, M Koga, T Maruyama, H Ogawa, M Saito, A Suzuki, F Tsushima

TOKAI U, HIRATSUKA - M Eto, M Koshiba, K Nishijima

OSAKA U - A Kusano, Y Nagashima, M Takita, T Yamaguchi

NIIGATA U - T Ishizuka, X Kitaguchi, H Koga, K Miyano, H Okazawa, M Takahata

TOKYO INST TECH - Y Hayato, Y Kanaya, K Kaneyuki,

$Y$ Watanabe

GIFU U - S Tasaka

MIYAGI U OF EDUCATION - M Mori

KOBE U - S Echigo, M Kohama, A T Suzuki

TOKYO U, INS -- E Ichihara, T Inagaki, K Nishikawa, A Sakai

BOSTON U - M Earl, E Kearns, S B Kim, M Messier, I L Stone,

L R Sulak, K Yoshida

BROOKHAVEN - M Goldhaber

UC, IRVINE - T Barszczak, W Gajewski, P G Halverson, J Hsu,

W R Kropp, L R Price, F Reines, H W Sobel

CAL STATE, DOMINGUEZ HILLS .. K Ganezer, W Keig

GEORGE MASON U - R W Ellsworth

IIAWAII U - J Flanagan, J Learned, S Matsuno, V Stenger

LOS ALAMOS - $\mathrm{T} \mathrm{J}$ Haines

LOUISIANA STATE U -. E Blaufuss, R Sanford, R Svoboda, $M$ R Vagins

MARYLAND U -- M L Chen, Z Conner, I A Goodman, G Sullivan SUNY, STONY BROOK .. J Hill, C K Jung, C Mauger,

C McGrew, B Viren, C Yanagisawa

WARSAW U - D Kielczewska

WASHINGTON U, SEATTLE *. V Chaloupka, J George, A Stachyra. L Wai, J Wilkes, K Young

Accelerator NONE Detector SUPER-KAMIOKANDE

Brief description Uscs a 50,000 -ton ring imaging water Cerenkov detector at a depth of $2700 \mathrm{~m}$ of water equivalent (mwe) in the Kamioka Mozumi mine in Japan. The detector consists of 


\section{SUMMARIES OF NONACCELERATOR EXPERIMENTS}

a stainless steel tank in the shape of a right circular cylinder, $39 \mathrm{~m}$ diameter and $41 \mathrm{~m}$ height, filled with purified water. It is optically segmented into an inner volume, and an outer (anti-coincidence) region. The inner region is viewed by 11,200 photomultiplier tubes (PMT's). The outer annulus is used to tag entering muons as well as to attenuate low-energy $\gamma$ 's and neutrons. The outer region is viewed by 1,800 PMT's. Taking data (July 96).

Related experiments KEK-362

E-mail contact totsuka@sukip04.icrr.u-tokyo.ac.jp WWW Home-page http://www-sk.icrr.u-tokyo.ac.jp/

\section{UNDERGROUND-KGF}

(Began data-taking Oct 1980, Completed data-taking 1992)

\section{THE KOLAR, GOLD FIELD EXPERIMENT}

TATA INST - H Adarkar, S R Dugad, S D Kalmani,

M R Krishnaswamy, J D Kulkarni, M G K Menon, N K Mondal,

P S Murty, P Nagaraj, V S Narasimham ( $\sqrt{ }$ Spokesperson),

B Satyanarayana, B V Sreekantan

OSAKA CITY U ... Y Hayashi, N Ito, S Kawakami, T Mitsuyama,

T Nakamura, $\mathrm{K}$ Tanaka

KANAGAWA U - S Miyake

Accelerator NONE Detector Calorimeter

Particles studied $p, n$

Brief description Phase-I of the experiment was completed in 1985. The phase-II detcctor is a 260 -ton iron tracking calorimeter with 60 layers of proportional counter tubes, $6600 \mathrm{~m}$ of water equivalent underground. A monopole detector has been added in phase-III. Studies nucleon decays and searches for magnetic monopoles and point sources of high-energy $\nu_{\mu}$ 's. Completed in 1992 .

Journal papers PL B106 (1981) 339, PL B115 (1982) 349, PL B142 (1984) 99, NC 9C (1986) 167, NIM A284 (1989) 422, and PL B267 (1991) 138. No further papers expected.

E-mail contact vsn@tifrvax.tifr.res.in

\section{UNDERGROUND-LVD}

(Proposed 1984, Approved Apr 1985, Began data-taking Jun 1992, In progress)

\section{SEARCH FOR STELLAR-COLLAPSE NEUTRINOS} WITH THE LARGE VOLUME DETECTOR

LVD COLLABORATION

ASHIKAGA INST TECH - K Saitoh

BOLOGN $\Lambda$ U - P Antonioli, G Bari, M Basile, F Bersani, G Bonoli, G Bruni, G Cara Romeo, L Cifarelli, F Cindolo, A Contin, L Emaldi, C Ghetti, P Giusti, F Grianti, G Iacobucci,

M L Luvisetto, T Massam, R Nania, A Pesci, G Sartorelli, M Selvi, A Zichichi ( $\sqrt{ }$ Spokesperson)

BROWN U-A Bosco, $A$ De Silva, M Widgoff

CAMPINAS U - J A Chincellato, $L$ G Dos Santos, E Kemp

N Mengotti Silva, A Turtelli

FRASCATI - F L Fabbri, G Maccarrone, L Votano

GRAN SASSO \& INFN, ASSERGI - N Taborgna

HOUSTON U - K Lau, B Mayes, L Pinsky, 3 Pyrlik, R Weinstein INDIANA U - E D Alyea

MIT, LNS - M Deutsch, E S Hafen, P Haridas, I A Pless, J Tang

NORTHEASTERN U - J Moromisato, E Von Goeler

MOSCOW, INR - V S Berezinsky, V L Dadykin, R I Enikeev,

F F Khalchukov, E V Korolkova, P V Kortchaguin,

V B Kortchaguin, V A Kudryavtsev. A S Malguin, V G Ryasny,

O G Ryazhskaya, V P 'Talochkin, V F Yakushev, G $T$ ' Zatsepin

OKAYAMA U - S Tsuji, T Wada

OKAYAMA UNIV SCI ... I Yamamoto

PERUGIA U \& INFN, PERUGIA - B Alpat, I Uman

SAITAMA U - N Inouc

TURIN, COSMO-GEOFISICA LAB \& TURIN U \& INFN,

TURIN - C Aglietta, G Badino, M Bertaina, R Bertoni,

C Castagnoli, A Castellina, A Chiavassa, W Fulgione,
P Galeotti, P Ghia, R Granella, C Melagrana, C Morello, G Navarra, L Panaro, L Periale, P Picchi, O Saavedra,

G C Trinchero, $P$ Vallania, $S$ Vernetto, C Vigorito

URBINO U \& INFN, FLORENCE - G Conforto, P Dominici,

G Guidi, R Mantovani, A Megna, S Santini, F Vetrano

Accelerator NONE Detector Scintillator, Streamer chamber

Reactions

$\bar{\nu}_{e} p \rightarrow e^{+} n$

$\nu \mathrm{C} \rightarrow \nu \mathrm{C} \gamma$

$\bar{\nu} \mathrm{C} \rightarrow \bar{\nu} \mathrm{C} \gamma$

$\nu e^{-} \rightarrow \nu e^{-}$

$\nu_{e} \mathrm{C} \rightarrow e^{-} \mathrm{Nit}$

$\bar{\nu}_{e} \mathrm{C} \rightarrow e^{+}$Bor

Particles studied $p, n$, muon, $\nu$

Brief description 'The experiment is located in the Gran Sasso Laboratory at a minimum depth of about $3300 \mathrm{mwe}$. The apparatus consists of a streamer tube tracking system interleaved with a large volume of liquid scintillator and its support structure which acts as a passive absorber. It is a high precision tracking calorimeter with the major part of its volumc sensitive, and with the sensitive elements uniformly distributed. Of the five towers which will constitute the complete LVD, the first one is operational since June 92 and the second one since June 94 . The main features of an LVD tower are: surface area $660 \mathrm{~m}^{2}$, geometrical acceptance $1768 \mathrm{~m}^{2} \mathrm{sr}$, and liquid scintillator mass 368 tons. The major purpose of the experiment is to search for neutrinos from stellar collapses in our galaxy. Other physics goals include: measurement of the atmospheric neutrino flux and search for neutrino oscillations, study of the spectrum and interactions of cosmic ray muons and muon bundles, and investigation of events detected in time coincidence with the EASTOP experiment at the surface of the mountain Taking data (July 96).

Journal papers NC C9 (1986) 237, NIM A264 (1988) 5, NIM A274 (1989) 177, NM A277 (1989) 11, NIM A277 (1989) 17 NIM A295 (1990) 466, NC 105A (1992) 1793, NC 105A (1992) 1815, NIM A329 (1993) 521, NP (PROC SUPPL) 31 (1993) 450, NP (PROC SUPPL) 35 (1994) 240, NP (PROC SUPPL) 35 (1994) 243, NP (PROC SUPPL) 35 (1994) 259, NP (PROC SUPPL) 35 (1994) 267, ASPP 2 (1994) 103, ASPP 3 (1995) 311 , and NC $18 \mathrm{C}$ (1995) 628

Related experiments MACRO, KAMIOKANDE, SUPERKAMIOKANDE

E-mail contact sartorelli@bo.infn.it

\section{UNDERGROUND-MACRO}

(Proposed 1984, Approved Apr 1985, Began data-taking Feb 1989. In progress)

\section{MONOPOLE, ASTROPHYSICS, AND COSMIC RAYS} OBSERVATORY

MACRO COLLABORATION

BARI U \& INFN, BARI - R Bellotti, F Cafagna, M Calicchio, $M$ Castellano, L De Benedictis, G De Cataldo, C De Marzo,

O Erriquez, C Favuzzi, P Fusco, N Giglietto, P Guarnaccia, $M$ N Mazziotta, $T$ Montaruli, $A$ Raino, P Spinelli

BOLOGNA U \& INFN, BOLOGNA - S Cecchini, H Dekhissi,

R Fantini, G Giacomelli ( $\checkmark$ Spokesperson), F Maaroufi,

G Mandrioli, S Manzoor, A Margiotta-Neri, L Patrizii, V Popa, P Serra-Lugaresi, M Spurio, V Togo

BOSTON U - E Kearns, C Okada, C Orth, I L Stone, L R Sulak

CAL TECH - B C Barish ( $\sqrt{ }$ Spokesperson), E Katsavounidis,

$S$ Kyriazopoulou, N Longley, D G Michael, R Nolty, C W Peck, K Scholberg, C W Walter

DREXEL U - C Lane, R Steinberg

FRASCATI - G Battistoni, H Bilokon, C Bloise, M Carboni.

V Chiarella, C Forti, E Iarocci, A Marini, V Patera, F Ronga,

L Satta, A Sciubba, M Spinetti

GRAN SASSO - R Antolini, A Di Credico, A Grillo, C Gustavino, S Mikheyev, S Parlati, J Reynoldson, E Scapparone INDIANA U $-\mathrm{C}$ Bower, A Ilabig. A Hawthorne, $\mathrm{R}$ Heinz,

L Miller, S Mufson, J Musse 


\section{SUMMARIES OF NONACCELERATOR EXPERIMENTS}

AQULLA U - I De Mitri, P Monacelli

LECCE U \& INFN, LECCE - P Bernardini, G Mancarella,

D Martello, O Palamara, S Petrera, P Pistilli, A Surdo

MICHIGAN U - R Baker, S Coutu, K Hanson, D Levin, M Longo, G Tarle

NAPLES U, IFS \& INFN, NAPLES - M Ambrosio

G C Barbarino, D Campana, F Guarino, G Osteria, U Rubizzo

PISA U \& INFN, PISA - A Baldini, C Bemporad, F Cei,

G Giannini, M Grassi, D Nicolo, R Pazzi

ROME U - G Auriemma, S Bussiro, M De Vincenzi, E Lamanna, P Lipari, C Satriano, M Severi

TEXAS $A$ AND M - A Sanzgiri, $R$ Webb

TURIN U \& INFN, TURIN - V Bisi, A Marzari-Chiesa, M Monteno, M Sitta

\section{Accelerator NONE Detector MACRO}

Particles studied monopole, muon, $\nu$

Brief description The MACRO detector has becn primarily designed to conduct a search for supermassive grand unified magnetic monopoles. It is a general purpose detector, which is also searching for nuclearites, WIMP's, fractional charge particles, $\overline{\nu_{e}}$ from stellar gravitational collapses, high-energy $\nu_{\mu}$ 's from cosmic sources, etc. It is studying high-energy cosmic ray muons (vertical intensity, seasonal variation, moon shadow, anisotropy, possible muon astronomy), cosmic ray composition at high energies, atmospheric neutrinos, etc. Operates in coincidence with an air shower array (EASTOP) to study the primary cosmic ray composition at high energies. The detector has six supermodules in two levels, each instrumented to operate independently of the others. Each lower supermodule consists of a horizontal array of two layers of liquid scintillation counters, ten layers of limited streamer tubes, one layer of CR39 nuclear track detectors and seven layers of absorbers. The upper part (Attico) has four horizontal layers of streamer tubes and one layer of scintillators. The sides are covered with one layer of scintillators and 6 layers of streamer tubes. The CR39 detector is also mounted on the east vertical side and on the north lower side. The global dimensions are $12 \times 76 \times 9 \mathrm{~m}^{3}$ and it contains 600 tons of liquid scintillator. The detector is located in Hall B of the Gran Sasso Laboratory. Has an overhead shielding of about $3800 \mathrm{~m}$ of water equivalent. Taking data in its full configuration (July 96).

Journal papers NC 9C (1986) 281, NIM A281 (1989) 213, PR D42 (1990) 1396, PL B249 (1990) 149, NIM A300 (1991) 581 NIM A301 (1991) 275, NP (PROC SUPPL) 24B (1991) 191, NIM A321 (1992) 609, PRL 69 (1992) 1860, ASPP 1 (1992) 11, PR D46 (1992) 895, PR D46 (1992) 4836, NP B370 (1992) 432 NIM A324 (1993) 337, ASTJ 412 (1993) 30, PL B337 (1994) 376, PRL 72 (1994) 608, PRL 73 (1994) 1306, PR D50 (1994) 3046, PL B357(1995) 481, ASPP 4 (1995) 33, and PR D52(1995) 3793

Related experiments CERN-WA-086, CERN-EMU-018

E-mazl contact giacomelli@bo.infn.it, barish@cithex.caltech.edu WWW Home-page http://rsgs02.Ings.infn.it/macro/macro.html

\section{UNDERGROUND-SAGE}

(Proposed 1964, 1984, Approved 1985, Began data-taking May 1988, In progress)

THE RUSSIAN-AMERICAN GALLIUM SOLAR NEUTRINO EXPERIMENT (SAGE)

SAGE COLLABORATION

MOSCOW, INR - J N Abdurashitov, V N Gavrin

( $V$ Spokesperson), S V Girin, $V \vee$ Gorbatchev, ' $T$ ' V Ibragimova,

A V Kalikhov, $N$ G Khairnasov, T V Knodel, I N Mirmov,

A A Shikhin, E P Veretenkin, V M Vermul, V E Yants,

G T Zatsepin

LOS ALAMOS - T J Bowles ( $\checkmark$ Spokesperson), J S Nico,

W A Teasdale, D L Wark

WASHINGTON U, SEATTLE - S R Elliott, J F Wilkerson

PENN U - B T Cleveland, 'T Daily, $R$ Davis, $K$ Lande, $C K$ Lee,

$P W$ Wildenhain

LOUISIANA STATE U - M L Cherry

Accelerator NONE Detector GGNT
Reactions

$$
\nu_{e}^{7 l} \mathrm{Ga} \rightarrow e^{-71} \mathrm{Ge}
$$

Particles studied $\nu_{e}$

Brief description Uses the Gallium-Germanium Neutrino Telescope (GGNT) situated in an underground laboratory built in the Baksan Neutrino Observatory, Northern Caucasus, Russia. Has an overhead shielding of about $4700 \mathrm{~m}$ of water equivalent. Sensitive to the low energy neutrinos produced by the $p p$ fusion in the Sun. Exploits the radiochemical procedure and uses liquid metallic gallium ( 30 tons in the first stage, 57 tons in 1991). A purification procedure, implemented beginning with the January 90 extraction, resulted in a significant background reduction. The SAGE-II phase began in September 92 . Counts the $K$ and $L$ peaks in ${ }^{71}$ Ge decay, with 57 tons of $\mathrm{Ga}$ and low background. $\Lambda$ calibration with a ${ }^{51} \mathrm{Cr}$ artificial neutrino source of about $0.5 \mathrm{mC}$ activity was carried out in 1995. Taking data (July 96).

Journal papers PRL 67 (2991) 3332, and PL B328 (1994) 234.

Related experiments GALLEX, BOREXINO

E-mail contact gavrin@adonis.iasnet.ru, tjb@lanl.gov

\section{UNDERGROUND-SOUDAN-2}

(Proposed 1981, Approved 1983, Began data-taking 1988. In progress)

\section{THE SOUDAN-2 PROTON DECAY EXPERIMENT}

ARGONNE - D S Ayres, T H Fields, M C Goodman, E N May, L E Price, R V Seidlein, J L 'Thron, J L Uretsky

MINNESOTA U - C R Bode, P M Border, H Courant,

D M DeMuth, R Gran, S M S Kasahara, N P Longley, M L Marshak ( $\checkmark$ Spokesperson), W H Miller, L Mualem, E A Peterson, K Ruddick, M II Schub, V Vassilicv, G Villaume, S Wakely

OXFORD U - W W M Allison, C B Brooks, J H Cobb,

H M Gallagher, D H Perkins, A Stassinakis, N West, U Wiclgoš RUIHERFORD - G J Alner, D J A Cockerill, R J Cotton, $P$ J Litchfield, G F Pearce

TUFTS U - B Ewen, T Kafka, W Leeson, W A Mann,

R H Milburn, A Napier, W Oliver, J Schneps, N Sundaralingam WESTERN WASHINGTON U - W L Barrett

Accelerator NONE Detector Calorimeter

Particles studied $p, n, \nu_{e}, \nu_{\mu}$

Brief description A 960-ton iron tracking calorimeter uses drift projection tubes arranged in a hexagonal array. The tubes are $15 \mathrm{~mm}$ in diameter separated by $1.6 \mathrm{~mm}$ of steel. Trigger thresholds are $100 \mathrm{MeV}$ kinetic energy for muons and $150 \mathrm{MeV}$ for clectrons. The main detcetor is completely surrounded by a $1700 \mathrm{~m}^{2}$ active shield of proportional tubes which identifies events associated with cosmic ray muons. A charged particle test-beam calibration of the 4.3-ton calorimeter modules has been completed. $\Lambda$ surface array and an air Cerenkov detector are operated in coincidence with the SOUDAN-2 detector to provide information about the air showers which produce underground muons. The experiment is located in the Soudan mine, Minnesota, $2090 \mathrm{~m}$ of water equivalent underground. The data taking began in mid-1988 when 275 tons of the detector were installed. The detector was completed in late 1993. Physics topics include studies of nucleon decay, atmospheric neutrinos and neutrino oscillations, cosmic ray composition, and searches for magnetic monopoles and point sources of cosmic rays. Taking data (July 96). The collaboration has also proposed the use of the SOUDAN-2 detector for a long baseline neutrino oscillation experiment MINOS (see FNAL-875).

Journal papers NIM A276 (1989) 371, NIM A283 (1989) 642, PR D42 (1990) 2967, JPHY G17 (1991) S393, PL B269 (1991) 220, NP (PROC SUPPL) 28A (1992) 377, PR D46 (1992) 4846, PR D52 (1995) 2760, and NIM A376 (1996) 36

Related experiments FNAL-822, FNAL-875

E-mail contact marshak@mnhepl.hep.umn.edu

WWW Home-page http://hepwww.rl.ac.uk/soudan2/index.html 


\section{SUMMARIES OF NONACCELERATOR EXPERIMENTS}

\section{UNDERGROUND-SUDBURY}

(Proposed 1985, Approved 1990, In preparation)

\section{THE SUDBURY NEUTRINO OBSERVATORY (SNO)}

\section{SNO COLLABORATION}

QUEENS U, KINGSTON - E P Bonvin, L Erhardt, H C Evans, G T Ewan ( $\checkmark$ Spokesperson), R J Ford, A L Hallin, A Hamer, C J Jillings, H W Lee, J R Leslie, J D MacArthur, H B Mak, A B McDonald ( $\checkmark$ Spokesperson), W McLatchie, B Moffat, $T \mathrm{~J}$ Radcliffe, $B$ C Robertson, $P$ Skensved, $R \mathrm{~L}$ Stevenson

CHALK RIVER, AECL - E D Earle, J D Hepburn, G Jonkmans, $B$ Su

CRPP, OTTAWA - W F Davidson, F Delnoki-Varess,

C K Hargrove, K McFarlane, T Noble, V M Novikov, M O'Neill, M Shatkay, D Sinclair

GUELPH U - T Anderson, $\mathrm{M}$ C Chon, $\mathrm{P}$ Jagam, J Law, R Ollerhead, J J Simpson, J X Wang

LAURENTIAN U - J Bigu, E D Hallman, R U Haq J G Hykaway, A Roberge, E Saettler, C J Virtue BRITISH COLUMBIA U - R Helmer, R Komar, A Poon, C Waltham

PENN U - E W Beier ( $\checkmark$ Spokesperson), D Cowen, W Frati, $J$ R Klein, D McDonald, F M Newcomer, $R$ Van de Water, $R$ Van Berg, J Wang, $P$ Wittich

LOS ALAMOS - T J Bowles, S J Brice, M M Fowler,

$A$ Goldschmidt, A Hime, $P$ Thornewell, J B Wilhelmy, J M Wouters

LBL - Y D Chan, M P Isaac, K T Lesko, M E Moorhead, E B Norman, A R Smith, R G Stokstad

WASHINGTON U, SEAT'ILE - Q R Ahmad, J Beck,

M C Browne, P J Doe, C Duba, S R Elliott, I V Germani, R Meijer-Drees, R G H Robertson, T D Steiger, J F Wilkerson OXFORD U - J C Barton, S Biller, M G Bowler, X Chen.

$G$ Doucas, H D Heron, N A Jelley ( $\checkmark$ Spokesperson), A B Knox, W J Locke, J J Lyon, N W Tanner ( $\checkmark$ Spokesperson), R K Taplin, M D Thorman, P T Trent, D L Wark, N West BROOKHAVEN .. J Boger, R L Hahn, J K Rowley

Accelerator NONE Detector Counter

\section{Reactions}

$$
\begin{aligned}
& \nu e^{-} \rightarrow \nu e^{-} \\
& \nu_{e} \text { deut } \rightarrow p p e^{-} \\
& \nu \text { deut } \rightarrow p n \nu \\
& \bar{\nu}_{e} \text { deut } \rightarrow n n e^{+} \\
& \bar{\nu}_{e} p \rightarrow n e^{+}
\end{aligned}
$$

Particles studied $\nu$

Brief description The detector is a 2000-ton heavy water $\left(\mathrm{D}_{2} \mathrm{O}\right)$

Cerenkov detector designcd to study neutrinos from the Sun and other astrophysical sources. The use of heavy water allow both electron neutrinos and all other types of neutrinos to be observed by thrce complementary reactions. The detector will be sensitive to the $\nu_{c}$ flux and energy spectrum shape and to the total neutrino flux irrespective of neutrino type. These measurements will provide information on both vacuum neutrino oscillations and matter-enlanced oscillations, the MSW effect. In the cvent of a supernova it will be very sensitive to $\nu_{\mu}$ and $\nu_{\tau}$ as well as the $\nu_{e}$ 's emitted in the initial burst, enabling sensitive mass measurements as well as providing details of the physics of stcllar collapse. The underground cavity is complete and cquipment is being installed. The detector is scheduled to bo filled with heavy water in Spring 1997. In preparation (July 96)

Journal papers NC 9C (1986) 308, PL B194 (1987) 321, NIM A314 (1992) 373, and NM A370 (1996) 579.

E-mail contact ewan@mips2.phy.queensu.ca, geneb@upenn5.hep.upenn.edu, n.jelley1@physics.oxford.ac.uk. n.tanner1@physics.oxford.ac.uk

WWW Home-page http://snodaq.phy.queensu.ca/SNO/sno.html

\section{UNDERICE-AMANDA}

(In preparation)

\section{ANTARCTIC MUON AND NEUTRINO DETECTOR:} AMANDA

AMANDA COLLABORATION

UC, BERKELEY - D Lowder, T Miller, P B Price, A Richards LBL - D Nygren

UC, IRVINE - S Barwick ( $\checkmark$ Spokesperson), P C Mock, R Porrata, E Schneider, G Yodh

WISCONSIN U - A Coulthard, K Engel, L Gray, F Halzen

( $\checkmark$ Spokesperson), I Jacobsen, V Kandhadai, I Liubarsky, $\mathrm{R}$ Morse, J C Spang, S Tilav

STOCKMOLM U .. P Askebjer, L Bergstrom, A Bouchta,

E Dahlberg, B Erlandsson, A Goobar, P O Hulth, S Johansson, Q Sun, C Walck

UPPSALA U - S Carius, A Hallgren, H Rubinstein

DESY-IFH, ZEUTHEN - H Heukenkamp, S Hundertmark,

A Karle, C Spiering, O Streicher, $\mathrm{T}$ Thon, $\mathrm{R} W$ ischnewski

BARTOL RESEARCH INST - T C Miller

Accelerator NONE Detector P.MT

Particles studzed $\nu$

Brief description AMANDA is a high-energy neutrino observatory which uses the deep South Pole ice as a target and a tracksensitive, transparent detecting medium. Upward moving neutrinos, having passed through the Earth, interact with icc and produce muons. The muons generate Cerenkov radiation which can be recorded by photomultiplier tubes (PMT). The tubes are placed in vertical shafts melted into the icecap to the depth of $1720 \mathrm{~m}$. The data acquisition is handled in a counting facility at the surface. As of July 96 , a total of 173 operating PMT's has been installed. The plan is to have at least 800 optical mod uies operating before the end of the century. Under construction (July 96).

Journal papers NATU 353 (1991) 807, and SCI 267 (1995) 1147 Related experiments BAIKAL, DUMAND, NESTOR

E-7nail contact sbarwick@uci.edu

halzen@phenxh.physics.wisc.edu

WWW Home-page http://dilbert.lbl.gov/www/ananda.html

\section{UNDERWATER-BAIKAL}

(Proposed 1984, Approved Jan 1987, Nov 1992, Began datataking 1993, In progress)

THE LAKE BAIKAL DEEP UNDERWATER NEUTRINO TELESCOPE, NT-200

BAIKAL COLLABORATION

MOSCOW, INR - L B Bezrukov, B A Borisovets,

I $\Lambda$ Danilchenko, Z A M Djilkibaev, G V Domogatsky

( $\checkmark$ Spokesperson), A A Doroshenko, A A Garus, A M Klabukov, S I Klimushin, B K Lubsandorzhiev, A I Panfilov,

D P Petukhov, P G Pokhil, I A Sokalski

IRKUTSK STATE U - N M Budnev, A G Chensky, V I Dobrynin, $O N$ Gaponenko, $T$ A Gress, $A$ P Koshechkin, $R R$ Mirgazov, A V Moroz, S A Nikiforov, Y V Parfenov, A A Pavlov.

K A Pocheikin, P A Pokolev, V Y Rubzov, S I Sinegovsky,

V A Tarashansky

MOSCOW STATE U - S B Ignat'ev, L A Kuzmichev, N I Moseiko, E A Osipova

NIZHINII NOVGOROD STATE U - S V Fialkovsky, V F Kulepov, M B Milenin

S'T PETERSBURG, MARINE TECII U - M I Rozanov

KURCHATOV INST, MOSCOW * A I Klimov

DUBNA -- I A Belolaptikov

DESY, ZEUTHEN - A Karle, T Mikolajski, D Pandel, C Spicring,

O Streicher, $\mathrm{T}$ Thon, C Wiebusch, $\mathrm{R}$ Wischnewski

Accelerator NONE Detector Counter

Partzcles studied $\nu$, muon, monopole

Brief description The deep-underwater Cerenkov detector N'T-

200 , effective area of about $2000 \mathrm{~m}^{2}$, will consist of 192 optical 


\section{SUMMARIES OF NONACCELERATOR EXPERIMENTS}

modules arranged on 8 strings at $1100 \mathrm{~m}$ depth. The modules are equipped with $37-\mathrm{cm}$ QUASAR phototubes. The experiment studies muons generated in neutrino interactions or in reactions of primary cosmic rays in the atmosphere, and searches for GUT magnetic monopoles. Measures the neutrino flux from the Earth atmosphere, scarches for neutrinos from cosmic sources and from WIMP annihilations in the center of the Earth. A part of the detector consisting of 36 optical modules has operated in 1993/94. A 72-module version took data from Apr 95 to Mar 96, and the 96-module detector (half of the NT-200) began taking data in Apr 96.

Journal papers NP (PROC SUPPL) $14 \mathrm{~B}$ (1990) 51, NP (PROC SUPPL) 19 (1991) 388, YF 52 (1990) $86=$ SJNP 52 (1990) 54 , NP (PROC SUPPL) $28 B$ (1992) 491, NP (PROC SUPPL) 35 (1994) 290, NP (PROC SUPPL) 35 (1994) 301, NP (PROC

SUPPL) 43 (1995) 241, and NP (PROC SUPPL) 48 (1995) 463.

Related experiments AMANDA, DUMAND, NESTOR

E-mail contact domogats@pcbai10.1pi.msk.su, csp@ifh.de WWW Home-page http://www.ifh.de/baikal/baikalhome.html

\section{UNDERWATER-DUMAND}

(Proposed 1988, Approved 1990, In preparation)

\section{DEEP UNDERWATER MUON AND NEUTRINO} TELESCOPE

DUMAND-II COLLABORATION

AACHEN. 'TECH HOCHSCH, III PHYS INST - P Bosetti, D Samm, C Wiebusch

BERN U -- P K F Gricder, P Minkowski, E Torrente-Lujan BOSTON $\mathbf{j}$ - S T Dye, E Hazen, A Mavretic

UC, BERKELEY - II Crawford, C Kuo, G Shapiro, I Stevenson UC, SAN DIEGO $\ldots \mathrm{H}$ Bradner

HAWAII U - J Bolesta, P W Gorham, S Kondo, J G Learned ( $\checkmark$ Spokesperson), S Matsuno, M Mignard, R Mitiguy,

D O'Connor, S Olsen, V Z Peterson, A Roberts, M Rosen, V J Stenger, D Takemori, G Wilkins

IOWA STATE U - E W Anderson, J M Hauptman, K Mauritz KIEL U - T Knutz, P Koske

KOBE U - K Kobayakawa

KINKI U, IIZUKA - T Kitamura

LOUISIANA STATE U - R Clark, R Svoboda, M Vagins

OKAYAMA UNIV SCI - I Yamamoto

SCRIPPS INST OCEANOGRAPHY - H Bradner

TOHOKU U - H Hanada, $T$ Hayashino, M Ito, Y Kawamorita,

H Kawamoto, $T$ Matsumoto, $\Upsilon$ Takayama, S Tanaka,

A Yamaguchi

TOKYO U, ICRR - T Aoki, K Mitsui, Y Ohashi, A Okada

KEK - M Fukawa, M Sakuda, S Uehara

VANDERBILT U ... K Miller, M Webster

WASHINGTON U, SEATTLE - H Berns, P Boynton,

V Chaloupka, B Egaas, J George, J J Lord, R J Wilkes, K Young

WISCONSIN U - U Camerini, W Grogan, M Jaworski, R March,

'T Narita, D Nicklaus

Accelerator NONE Detector Counter

Particles studied muon, $\nu$, monopole

Brief description In the first stage of the experiment

(DUMAND-I), a test of the operation of 7 phototube modules

was carried out. Measurements were made with a vertical string of modules suspended from a ship. Phase-Ir was approved in 1990. The plans called for an octagonal 9-string array, 24 tubes per string, to be built by 1995 . The array called DUMAND-II was to be located at a depth of $4760 \mathrm{~m}, 25 \mathrm{~km}$ off the coast of the Hawaiian Islands. For more details see the DUMAND-II proposal (U. of Hawaii report, HDC-1-88). The aim of the experiment is to build a system capable of searching for point sources of high-energy neutrinos of astrophysical origin, and very-highenergy cosmic ray muons. Other systems to be studied include WIMP's, quark nuggets, and monopoles. The detector is a 2megaton Cerenkov counter, with a muon area of $20,000 \mathrm{~m}^{2}$, and an angular resolution of $1^{\circ}$. Initial installation took place in December 93, and proof data was acquired. As of June 94, three strings are ready to be installed. However, a SAGENAP report of June 96 recommends cancellation of funding for the project, despite endorsement of its goals and feasibility. Other sources of support are being sought (July 96).

Journal papers NIM A276 (1989) 359, and PR D42 (1990) 3613.

Related experiments BAIKAL, AMANDA, NESTOR

E-mail contact jgl@uhhepg.phys.hawaii.edu

WWW Home-page http://web.phys.washington.edu

/local_web/dumand/aaa_dumand_home.html

\section{UNDERWATER-NESTOR}

(In preparation)

NESTOR: A NEUTRINO PARTICLE ASTROPHYSICS UNDERWATER LABORATORY FOR THE MEDITERRANEAN

NESTOR COLLABORATION

Accelerator NONE Detector Counter

Particles studied $\nu$

Brief description NESTOR is a detector for underwater neutrino astrophysics. It is located in the international waters off the southwest coast of Greece. The first phase of the project calls for the construction and deployment of a hexagonal tower with 168 optical modules and an effective area of $20,000 \mathrm{~m}^{2}$ suitable for the detection of TeV neutrinos. When completed, the full NESTOR array will have six additional towers in a hexagonal configuration, providing a sensitive area bigger than $100,000 \mathrm{~m}^{2}$ for $1 \mathrm{TeV}$ neutrinos, and an overall angular resolution better than $1^{\circ}$. The first tower is under construction and is expected to be deployed in 1997. For further information about the project please contact the Spokesperson, Prof. Leonidas K. Resvanis [Athens U.]

Related experiments BAIKAL, DUMAND, AMANDA

E-mail contact resvanis@grphla.hepl.uoa.ariadne-t.gr WWW Home-page http://abyss.hepluoa.ariadne-t.gr 